\title{
Stability Analysis and Optimal Control of Multi-server Wireless Queueing Systems
}

\author{
by \\ Hassan Halabian, M.A.Sc.
}

Doctor of Philosophy in Electrical and Computer Engineering

Ottawa-Carleton Institute for Electrical and Computer Engineering (OCIECE)

Department of Systems and Computer Engineering

Carleton University

Ottawa, Ontario, Canada, K1S 5B6

August, 2012

(C)Copyright 2012, Hassan Halabian 
Library and Archives

Canada

Published Heritage

Branch

395 Wellington Street

Ottawa ON K1A ON4

Canada
Bibliothèque et

Archives Canada

Direction du

Patrimoine de l'édition

395 , rue Wellington

Ottawa ON K1A ON4

Canada
Your file Votre référence

ISBN: 978-0-494-93657-3

Our file Notre référence

ISBN: $978-0-494-93657-3$
NOTICE:

The author has granted a nonexclusive license allowing Library and Archives Canada to reproduce, publish, archive, preserve, conserve, communicate to the public by telecommunication or on the Internet, loan, distrbute and sell theses worldwide, for commercial or noncommercial purposes, in microform, paper, electronic and/or any other formats.

The author retains copyright ownership and moral rights in this thesis. Neither the thesis nor substantial extracts from it may be printed or otherwise reproduced without the author's permission.
AVIS:

L'auteur a accordé une licence non exclusive permettant à la Bibliothèque et Archives Canada de reproduire, publier, archiver, sauvegarder, conserver, transmettre au public par télécommunication ou par l'Internet, prêter, distribuer et vendre des thèses partout dans le monde, à des fins commerciales ou autres, sur support microforme, papier, électronique et/ou autres formats.

L'auteur conserve la propriété du droit d'auteur et des droits moraux qui protege cette thèse. $\mathrm{Ni}$ la thèse ni des extraits substantiels de celle-ci ne doivent être imprimés ou autrement reproduits sans son autorisation.
In compliance with the Canadian Privacy Act some supporting forms may have been removed from this thesis.

While these forms may be included in the document page count, their removal does not represent any loss of content from the thesis.
Conformément à la loi canadienne sur la protection de la vie privée, quelques formulaires secondaires ont été enlevés de cette thèse.

Bien que ces formulaires aient inclus dans la pagination, il n'y aura aucun contenu manquant. 


\section{Abstract}

In this thesis, we concentrate on the stability analysis and stochastic control of multiserver wireless queueing systems. The queueing systems considered in this thesis can be applied to model orthogonal resource allocation in wireless access and satellite communication networks. Most of the work in this area has focused on deriving the optimal server allocation policy (optimality with respect to various objective functions), while only a few papers characterize the stability region of the studied models in an explicit form. An explicit characterization of the stability region not only enables us to solve network utility optimization problems with incurring less queueing delay, but also can facilitate the performance evaluation of the proposed control policies in network stochastic control. In the first part of this thesis, we focus on the stability analysis of three specific multi-server queueing systems, namely "multi-interface queueing system with a randomly connected server bank", "multi-queue multi-server system with random connectivities" and "multi-server single-interface queueing system with random connectivities". For each system, the stability region of the system is characterized by a finite set of linear inequalities. More specifically, for each queueing model we derive the necessary and sufficient conditions for the stability of the system for general packet arrival processes. For stationary arrivals, it is shown that these conditions will characterize the network stability region. For each system, we also derived an upper bound, in a closed form expression, for the average queueing delay of the optimal policy.

Thereafter, we generalize our analysis for the multi-queue multi-server system to a system with stationary $M$-ary channels (instead of independent binary channels) and derive a linear algebraic characterization of the stability region for this system. We further generalize the analysis for a fluid flow system and show that in this case, the stability region is determined by an infinite set of linear inequalities. However, by use of an example we show that depending on the channel distribution and the

number of queues, we may be able to characterize the stability region by a finite set 
of non-linear inequalities instead of infinite number of linear inequalities.

Finally, in the last part of the thesis we investigate the problem of delay-optimal server assignment in the multi-server single-interface queueing system for which we did the stability analysis in the previous part of the thesis. In particular, we consider the assignment of $K$ identical servers to a set of $N$ parallel queues in a time slotted queueing system with random connectivities. It has been previously proven that Maximum Weighted Matching (MWM) is a throughput-optimal server assignment policy for such queueing systems. In this thesis, we prove that for a symmetric system with i.i.d. Bernoulli packet arrivals and connectivities, MWM minimizes, in stochastic ordering sense, a broad range of cost functions of the queue lengths including total queue occupancy (or equivalently minimizes the average queueing delay). Then, we extend the model by considering imperfect services where it is assumed that the service of a scheduled packet fails randomly with a certain probability. We prove that the same policy is still optimal for the extended model. We also show that the results are still valid for more general connectivity and arrival processes, e.g., Poisson arrival process. 
To my wonderful wife, Oreyhanch, and beloved
family 


\section{Acknowledgments}

I am heartily thankful to my supervisors Professor Ioannis Lambadaris and Professor Chung-Horng Lung for their tremendous time and efforts spent in leading, supporting and encouraging me in the course of my thesis and study. This dissertation would not have been possible without their constant help and invaluable guidance throughout my study. I appreciate all their support and efforts to make my $\mathrm{Ph} . \mathrm{D}$. experience fruitful and a memorable phase of my life.

I also would like to express my gratitude to Professor Richard Yu for his precious time and valuable suggestions offered throughout parts of this work. Many thanks to my colleagues for their understanding and friendship during the time we spent together.

Last but not least, I would like to express my special thanks to my loving and supportive wife, Reyhaneh, for her unending inspiration, my mother, my brothers and my sister for their continuous understanding, supporting and encouraging in my life.

Thank you all! 


\section{Table of Contents}

$\begin{array}{ll}\text { Abstract } & \text { iii }\end{array}$

Acknowledgments $\quad$ vi

Table of Contents vii

List of Tables $\quad \mathbf{x i}$

List of Figures $\quad$ xii

List of Abbreviations xiv

List of Symbols $\quad$ Xv

1 Introduction 1

1.1 Research Overview ..................... 1

1.2 Thesis Focus . . . . . . . . . . . . . . . . . 4

1.3 Research Motivation and Thesis Contributions . . . . . . . . . . 5

1.3.1 Contributions to the Literature . . . . . . . . . . . . . 8

1.4 Notation . . . . . . . . . . . . . . . . . . . . 9 9

1.5 Thesis Organization . . . . . . . . . . . . . . 10

2 Background and Related Work $\quad 12$

2.1 Strong Stability Definition . . . . . . . . . . . . . . 13

2.2 Stability Region and Throughput-Optimal Server Allocation Policy . 14

2.2.1 Model Description-The General Queueing Network Case . . . 19

2.2.2 Implicit Characterization of the System Stability Region . . . 20

2.2.3 Back-pressure Algorithm . . . . . . . . . . . . . 23

2.3 Explicit Characterization of the Stability Region . . . . . . . . 25 
2.3.1 Utility Optimization Problems . . . . . . . . . . . . 25

2.4 Wireless Queueing Systems . . . . . . . . . . . . . . 26

2.4.1 Multi-server Wireless Queueing Systems _. . . . . . . . . 28

2.4.2 Stability Analysis in Wireless Queueing Systems . . . . . . . 29

2.5 Delay-Optimal Server Allocation in wireless Queueing systems . . . 30

2.6 Summary . . . . . . . . . . . . . . . . . 33

3 Stability Analysis of Randomly Connected Multi-server Queueing Systems

3.1 Multi-interface Queueing System with a Randomly Connected Server Bank . . . . . . . . . . . . . . 35

3.1 .1 System Description . . . . . . . . . . . . . . 35

3.1 .2 System Stability Analysis . . . . . . . . . . . . . 37

3.2 Multi-queue Multi-server System with Random Connectivities . . . . 40

3.2 .1 System Description . . . . . . . . . . . . . . . . 41

3.2 .2 System Stability Analysis . . . . . . . . . . . . . 42

3.3 Multi-server Single-interface Queueing System with Random Connec-

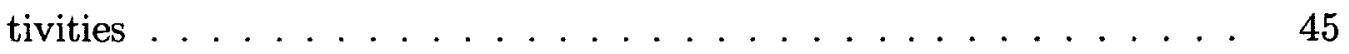

3.3.1 System Description . . . . . . . . . . . . . . 45

3.3.2 System Stability Analysis _ . . . . . . . . . . . 47

3.4 Numerical Results . . . . . . . . . . . . . . . . . . . . 54

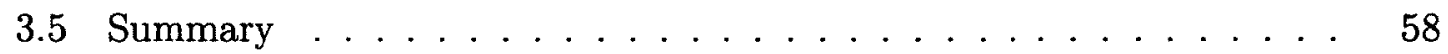

4 Explicit Characterization of Stability Region for Stationary MultiQueue Multi-Server Systems $\quad 59$

4.1 System Description . . . . . . . . . . . . . . . . . 60

4.2 Linear Algebraic Representation of Stability Region Ploytope . . . . 62

4.2.1 Region of Achievable Service Rates . . . . . . . . . 62

4.2.2 Necessary Conditions for the Stability of MQMS System . . . 63

4.2.3 Sufficient Conditions for the Stability of MQMS System . . . 70

4.2 .4 Numerical Example . . . . . . . . . . . . . . . . . . 71

4.2.5 Stability Region for Fluid Model MQMS Systems with Stationary Continuous Channel Distribution . . . . . . . . 77

4.3 Summary . . . . . . . . . . . . . . . . . . 79 
5 Optimal Server Assignment in Symmetric Multi-server Singleinterface Queueing Systems with Random Connectivities $\quad \mathbf{8 0}$

5.1 System Description . . . . . . . . . . . . . . . . 81

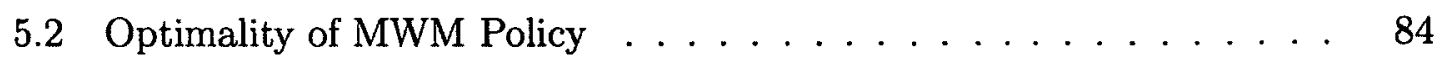

5.2.1 Stochastic Ordering and Dynamic Coupling . . . . . . . . 84

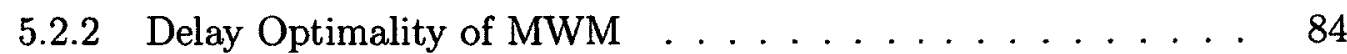

5.2 .3 Extensions . . . . . . . . . . . . . . . . . . . . 89

5.3 Simulation Results . . . . . . . . . . . . . . . 90

5.4 Summary $\ldots \ldots \ldots \ldots \ldots \ldots \ldots \ldots \ldots$

6 Conclusions and Suggestions for Future Research 97

6.1 Conclusions . . . . . . . . . . . . . . . . 97

6.2 Future Directions . . . . . . . . . . . . . . . . . 98

$\begin{array}{ll}\text { List of References } & 101\end{array}$

$\begin{array}{ll}\text { Appendix A Mathematical Background } & 108\end{array}$

A.1 Lyapunov Drift . . . . . . . . . . . . . . . . . . . . 108

A.2 Fundamental Concepts of Polytopes . . . . . . . . . . . . . . 109

Appendix B Proofs of the Results of Chapter 3 111

B.1 Proof of Lemma $3.1 \ldots \ldots \ldots \ldots$. . . . . . . . . . . . 111

B.2 Proof of Lemma $3.2 \ldots \ldots \ldots \ldots \ldots \ldots \ldots \ldots$

B.3 Proof of Lemma $3.3 \ldots \ldots \ldots \ldots \ldots$

B.4 Proof of Lemma $3.5 \ldots \ldots \ldots \ldots \ldots$

B.5 Proof of Lemma $3.6 \ldots \ldots \ldots \ldots$

B.6 Proof of Lemma $3.7 \ldots \ldots \ldots \ldots \ldots \ldots \ldots \ldots$

B.7 Proof of Lemma $3.8 \ldots \ldots \ldots \ldots \ldots \ldots \ldots \ldots$

B.8 Proof of Lemma $3.9 \ldots \ldots \ldots \ldots \ldots \ldots \ldots \ldots$

B.9 Proof of Lemma $3.10 \ldots \ldots \ldots \ldots \ldots$

Appendix C Proofs of the Results of Chapter 4 134

C.1 Proof of Lemma $4.5 \ldots \ldots \ldots \ldots \ldots \ldots$

C.2 Proof of Lemma $4.6 \ldots \ldots \ldots \ldots \ldots \ldots$

C.3 Proof of Theorem $4.2 \ldots \ldots \ldots \ldots \ldots \ldots$ 
Appendix D Proofs of the Results of Chapter $5 \quad 143$

D.1 Proof of Lemma $5.1 \ldots \ldots \ldots \ldots$. . . . . . . . . . 143

D.2 Proof of Lemma $5.2 \ldots \ldots \ldots \ldots \ldots \ldots$

D.3 Proof of Lemma $5.3 \ldots \ldots \ldots \ldots \ldots \ldots$

D.4 Proof of Lemma 5.3 for the System with Random Service Failures . . 152 


\section{List of Tables}

$4.1|\widehat{V}|$ and $\left|\mathcal{W}^{N}\right|-1$ for $N=2,3$ and $M=1,2,3,4 \ldots \ldots \ldots 69$ 


\section{List of Figures}

1.1 Two-queue single-server example . . . . . . . . . . . . . . . 2

2.1 Single-queue single-server system . . . . . . . . . . . . . 13

2.2 Single-queue, single-server system with random connectivity . . . . . 14

2.3 Two-queue single-server system with random connectivities . . . . . . 15

2.4 Stability regions of LCQ, Borst and $\operatorname{Max} \mu_{n}(t) / r_{n}(t)$ policies . . . . . 17

2.5 Delay comparison of $\mathrm{LCQ}$ with frequent and infrequent queue measurement . . . . . . . . . . . . . . . . . . . 31

3.1 Multi-interface queueing system with a server bank and random connectivities ..................... . . 36

3.2 Multi-queue multi-server queueing system with random connectivities 42

3.3 Two-queue single-server example . . . . . . . . . . . . . . . . . . 51

3.4 Stability region for the queueing system of Figure $3.3 \ldots \ldots 1$

3.5 Bipartite graph corresponding to problem (3.34) . . . . . . . . . 53

3.6 Stability regions of studied multi-server queueing systems for sample values of $N, K, R \ldots \ldots \ldots \ldots \ldots \ldots \ldots$

3.7 Evaluation of the obtained upper bounds . . . . . . . . . . . 56

3.8 throughput-optimal policy vs. randomized policy . . . . . . . . 57

4.1 Multi-queue multi-server queueing system with stationary channel distribution . . . . . . . . . . . . . . . . . 61

4.2 Stability region for $N=2,3, M=1,2,3,4$ and $K=3 \ldots \ldots$. . . 72

4.3 Stability region for $N=2, K=3, M=3$ with a random channel distribution (the optimal solution and the solution of CLC2b algorithm

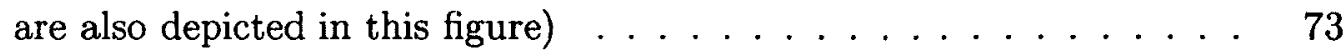

4.4 CLC2b algorithm framework . . . . . . . . . . . . . . . 75

4.5 Comparison of the performances of CLC2b algorithm with the optimal solution ........................ 76

4.6 Stability region for $\mu_{1}=2$ and $\mu_{2}=1 \ldots \ldots \ldots$. . . . . . 79 
5.1 Discrete time queueing system with $N$ parallel queues and $K$ servers

5.2 Examples of balancing server reallocations (the weight $c_{n, k}(t) x_{n}(t-1)$ of each edge $(n, k)$ is also shown) $\ldots \ldots \ldots \ldots \ldots$

5.3 Average total queue occupancy, $N=8, K=4, q=0.8 \ldots \ldots \ldots 94$

5.4 Average total queue occupancy, $N=8, K=8, q=0.8 \ldots \ldots \ldots .95$

5.5 Average total queue occupancy, $N=8, K=6, p=0.5 \ldots \ldots \ldots 96$

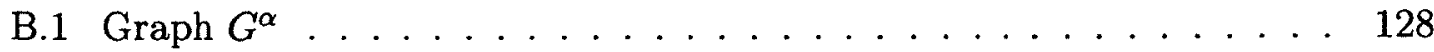

D.1 The negative cycle $\ell^{\star} \ldots \ldots \ldots \ldots \ldots \ldots \ldots \ldots \ldots$

D. 2 Cases 2 and 3 in the proof of Lemma $5.2 \ldots \ldots \ldots \ldots$ 


\section{List of Abbreviations}

CDMA Code Division Multiple Access

CLC2b Cross Layer Control-2b

IP Integer Programming

LCQ Longest Connected Queue

MAC Medium Access Control

MB Most Balancing

MQMS Multi-Queue Multi-Server

MM Maximum Matching

MW Maximum Weight

MWM Maximum Weighted Matching

MTLB Maximum-Throughput Load-Balancing

OFDM Orthogonal Frequency Division Multiplexing

OFDMA Orthogonal Frequency Division Multiple Access

SINR Signal to Interference plus Noise Ratio 


\section{Frequently Used Symbols}

$A_{n}(t) \quad$ Arrival random variable to queue $n$.

$A(t) \quad$ Arrival vector.

$C_{n}(t) \quad$ Connectivity of queue $n$.

$C(t) \quad$ Connectivity matrix (vector).

$X_{n}(t) \quad$ Length of queue $n$.

$X(t) \quad$ Queue length vector.

$\mu(t) \quad$ Service process.

$I(t) \quad$ Vector of resource allocation variables.

$\mathcal{N} \quad$ The set of queues.

$N \quad$ Number of queues.

$\mathcal{K} \quad$ The set of servers.

$K \quad$ Number of servers.

$R \quad$ Number of interfaces.

$\lambda \quad$ Arrival rate matrix (vector).

$p \quad$ Probability of connectivity in the single-queue single-server system.

$Q_{a}^{(c)} \quad$ Queue of commodity $c$ at node $a$.

$X_{a}^{(c)}(t) \quad$ Length of $Q_{a}^{(c)}$ at time slot $t$.

$\lambda_{a}^{(c)} \quad$ Exogenous arrival rate to $Q_{a}^{(c)}$.

$C_{a, b}(t) \quad$ Capacity of link $(a, b)$ at time slot $t$.

$S(t) \quad$ Topology state at time slot $t$.

$P(s) \quad$ Topology probability distribution.

$\Lambda_{\pi} \quad$ Stability region of policy $\pi$.

$\Lambda \quad$ System stability region.

$\pi \quad$ A policy.

$\Gamma \quad$ The set of achievable rate matrices.

$G \quad$ A transmission rate matrix.

$f_{a b}^{(c)} \quad$ Multi-commodity variables.

$\mathcal{S} \quad$ Topology state space.

$M \quad$ Maximum number of successful packet transmissions over $M$-ary channels. 
$W_{a, b}^{\star}(t) \quad$ Optimal weight associated to link $(a, b)$.

$I^{\star}(t) \quad$ Optimal resource allocation.

$f_{n}(r) \quad$ Utility function.

$r_{n} \quad$ Admitted traffic rate to queue $n$.

$\mathbb{Z}_{+} \quad$ Non-negative integers.

$A_{m a x}^{2} \quad$ Supremum of second moment of arrival random variables.

$p_{n} \quad$ Probability of connectivity of queue $n$.

$I_{j, k}^{(n)}(t) \quad$ Indicator variable for allocation of server $k$ to interface $j$ of queue $n$.

$H_{n}(t) \quad$ Random variable of departures from queue $n$.

$E_{i}^{Q} \quad$ Event that at least $i$ queues from subset $Q$ are connected.

$I_{n, k}(t) \quad$ Indicator variable for allocation of server $k$ to queue $n$.

$H_{n, k}(t) \quad$ Random variable of departures from queue $n$ by server $k$.

$p_{n, k} \quad$ Probability of connectivity of queue $n$ to server $k$.

$P(c) \quad$ Probability distribution of the connectivity process.

$\mathcal{C} \quad$ Connectivity process state space.

$I_{n, k}^{(\pi)}(t) \quad$ Indicator variables of policy $\pi$ for allocation of server $k$ to queue $n$.

$\mathcal{G} \quad$ The set of deterministic policies.

$g \quad$ A deterministic policy.

$\mathcal{I} \quad$ Allocation matrix state space.

$I^{(g)} \quad$ Mapping of policy $g$ from channel state to the allocation matrices space.

$\mathcal{R} \quad$ Region of achievable rates.

$\mathcal{M} \quad$ State space of the channel capacity of a link in MQMS system.

$C_{s} \quad$ Channel state matrix associated to the channel state $s$ in MQMS system.

$\mathcal{P} \quad$ Stability region polytope.

$V \quad$ A set of vectors in $\mathbb{R}_{+}^{N}$ defined in Chapter 4.

$\widehat{V} \quad$ A finite subset of set $V$ defined in Chapter 4.

$\mathcal{W} \quad$ A finite set of integers defined in Chapter 4.

$\mathcal{W}^{N} \quad$ The set of $N$-dimensional integer vectors whose elements belong to $\mathcal{W}$.

$\{0,1\}^{N} \quad$ The set of $N$-dimensional binary vectors.

$\pi_{s} \quad$ Probability of channel state $s$ in MQMS system.

$f_{C(t)}(c) \quad$ Probability distribution of continuous channel variables.

$\mathbb{R}_{+} \quad$ The set of non-negative real numbers.

$\mathbb{R}_{+}^{N} \quad$ Non-negative orthant.

$q \quad$ Probability of successful service.

$\mathcal{F} \quad$ The class of cost functions.

$f(\cdot) \quad$ A real function that belongs to $\mathcal{F}$.

$\leq_{s t} \quad$ Stochastic ordering.

$\preceq \quad$ A relation defined in Chapter 5 . 
$\preceq_{p} \quad$ Partial ordering defined in Chapter 5 .

$\mathbb{Z}_{+}^{N} \quad$ The set of $N$-dimensional non-negative integers.

$\mathrm{MW}_{\pi}(t) \quad$ Matching weight of policy $\pi$ at time slot $t$.

$\Pi^{M W M} \quad$ The set of policies that employ MWM at all time slots.

$\Pi_{t} \quad$ The set of policies that employ MWM until time slot $t$.

$h_{t}^{\pi} \quad$ Number of balancing reallocations required to convert $\pi$ to MWM at time $t$.

$Q_{n, k}(t) \quad$ Random variable of successful service.

$I^{(H)}(t) \quad$ The matching associated to the heuristic policy.

$I^{(M M)}(t)$ The matching associated to the Maximum Matching policy. 


\section{Chapter 1}

\section{Introduction}

\subsection{Research Overview}

Optimal stochastic network control is one of the primary goals in the design of emerging wireless networks. The main purpose of stochastic control in wireless networks is to enable cross-layer designs to achieve stochastically optimal resource allocation in the physical and MAC layers, coupled with flow control/utility optimization strategies in the transport layer and routing in the network layer $[1,2]$. Examples of network resources in the MAC and physical layers are transmission power, transmitter interfaces, OFDM sub-carriers, TDMA time frames, CDMA codes and any other type of shared resources, e.g., relay stations in relaying networks. In cross-layer network design, the operations of different layers are not independent and each layer may use the parameters of other layers to optimize its performance [1,2]. For example, the resource allocation in the physical and MAC layers can be optimized by using the queue length information which is a parameter of the network layer.

In general, a wireless network can be modeled by a queueing system in which the wireless users are modeled by a set of queues and the shared network resources are modeled by a set of servers with time varying service processes. Therefore, the resource allocation problem in wireless networks may be modeled by a server allocation problem in a queueing system. To clarify the concept of cross-layer design and server allocation in queueing systems we will study the following example.

Example: Consider a time slotted, two-queue, single-server system with arrival processes $A_{1}(t)$ and $A_{2}(t)$ representing the number of packet arrivals to queue 1 and queue 2 during time slot $t$, respectively. Assume that $A_{n}(t), n=1,2$, are i.i.d. Bernoulli distributed random variables for all $t$. We also assume that a single server 


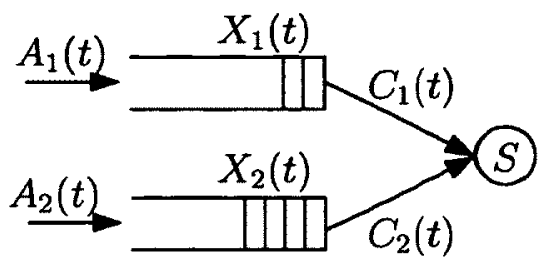

Figure 1.1: Two-queue single-server example

is shared between the queues to serve them during the operation of the system. However, the server can serve at most one packet from one queue per time slot. The queues can be assumed to model wireless users and the server may be assumed to model a frequency sub-channel. The queues are not always connected to the server and the connectivity of the queues to the server is changing randomly over time slots. We denote the connectivities of queue 1 and 2 to the server at time slot $t$ by $C_{1}(t)$ and $C_{2}(t)$, respectively (See Figure 1.1). Note that the connectivity of a queue to the server is analogous to the operation of a wireless channel whose connectivity is changing over time due to various factors such as mobility, noise, fading, etc. We assume that $C_{n}(t), n=1,2$ are i.i.d. Bernoulli distributed random variables for all $t$. Since the server is shared, we must exploit a server allocation scheme that allocates the server (i.e., the network resource) to the queues at each time slot. We define $I(t)$ to be 1 (to be 0 ) if the server is allocated to queue 1 (queue 2) at time slot $t$. Let $X(t)=\left(X_{1}(t), X_{2}(t)\right)$ be the queue length vector at the end of time slot $t$ after adding the arrivals of time slot $t$. Therefore, the queue length evolution for this example would be

$$
\begin{aligned}
& X_{1}(t+1)=\max \left\{X_{1}(t)-I(t) C_{1}(t), 0\right\}+A_{1}(t), \\
& X_{2}(t+1)=\max \left\{X_{2}(t)-(1-I(t)) C_{2}(t), 0\right\}+A_{2}(t) .
\end{aligned}
$$

A server allocation policy has to decide about the indicator variable $I(t)$ at each time slot. In [3], it has been proven that the server allocation policy that allocates the server to the Longest Connected Queue (LCQ) at each time slot will minimize the average total queue occupancy (or equivalently average queueing delay). Note that in this example, the stochastic objective function is to minimize the average queueing delay and the optimal server allocation policy (i.e., LCQ policy) is a cross-layer policy. This is due to the fact that in LCQ policy which is a resource allocation policy in the 
MAC layer we use the queue length information which in turn is a parameter of the network layer.

Various stochastic performance objectives can be considered in the stochastic control (optimization) of wireless networks. Examples of such objectives are stable throughput region, power consumption, utility functions of the admitted traffic rates, network life time and average queueing delay [1-37]. The first and maybe the main performance objective that must be considered in the stochastic control of any network is the stability region or network capacity region $[1,3-5,16,17,38]$. Stability region of a network is defined as the closure of the set of all the traffic arrival rate vectors for which there exists a network control strategy that can stabilize the network. Stability of a network intuitively means that the queue lengths do not tend to infinity as time progresses. In Chapter 2, queue stability and network stability are defined and these definitions are used throughout the thesis. Obviously, the network control strategies with larger stability regions are preferable since we can support more arrival rates stably. Stability region is also important in solving network utility optimization problems in the transport layer where the rate control (flow control) strategies are operating. In Chapter 2, network flow control problems are reviewed and it is shown that to address this issue in the transport layer we have to solve network utility optimization problems (which are convex programs). Although there exist online distributed algorithms that can solve these types of problems $[23,38,39]$, by having an explicit characterization of the stability region we can solve these problems offline and with incurring less buffering (delay) overhead. Moreover, an explicit characterization of the stability region is useful in performance evaluation of network control strategies in wireless networks [40-43]. The focus of the first part of this thesis which is presented in Chapters 3 and 4 is on the explicit characterization of the stability region for multi-server wireless queueing systems.

In the second part of the thesis in Chapter 5, we consider the problem of optimal server allocation in a multi-server queueing system with random connectivities and with the objective of minimizing, in a stochastic sense, the total queue occupancy (and hence queuing delay). Note that maximizing the stability region of a queueing system does not directly translate into minimizing the average queueing delay. This fact is shown in Section 2.5 of Chapter 2. In fact, not all the throughput-optimal resource allocation policies are delay-optimal. Our main contribution in Chapter 5 is to prove, using stochastic coupling argument, that for a symmetric system with 
i.i.d. Bernoulli packet arrival and connectivity processes, the Maximum Weighted Matching policy, a policy whose throughput optimality has been proven before in [5], is also delay-optimal.

\subsection{Thesis Focus}

In this thesis, we focus on the stability analysis and stochastic control of multi-server wireless queueing systems. The multi-server queueing systems studied in this thesis are applied to model the resource allocation problem in realistic wireless networks. The first part of our analysis in multi-server queueing systems (which includes the results of Chapters 3 and 4 ) is dedicated to stability analysis of a series of multi-server wireless queueing systems, namely:

- Multi-interface Queueing System with a Randomly Connected Server Bank,

- Multi-queue Multi-server (MQMS) System with Random Connectivities,

- Multi-server Single-interface Queueing System with Random Connectivities.

In all the systems studied in Chapter 3, the connectivities of the queues to the servers are assumed to be randomly varying over time slots (similar to the two-queue singleserver system mentioned in the previous section with ON-OFF channels). Each system is described in detail in Chapter 3 and then for each system we obtain the necessary and sufficient conditions for the stability of the system. From those conditions, the stability region of the system is concluded. For each system, we also introduce an upper bound for the total average queue occupancy (or average queueing delay).

In Chapter 4, the results of "Multi-queue Multi-server system" studied in Chapter 3 are extended to more general stationary channel processes (and not just ON-OFF channels). For this case, the stability region is specified by a finite set of linear inequalities. First, it will be shown that the stability region is a polytope characterized by the finite set of its facet-defining hyperplanes. Then, the coefficients of the linear inequalities describing the facet-defining hyperplanes of the stability region polytope are explicitly determined. Using a similar approach, the stability region for a fluid model system is characterized. However, the stability region of the fluid model system is described by an infinite number of linear inequalities since in this case the stability region is a convex surface. By an example, it will be shown that in some cases 
depending on the channel distribution, the stability region can be characterized by a finite set of non-linear inequalities instead of an infinite number of linear inequalities.

In the second part of our analysis, we will consider the problem of delay-optimal server allocation in the "Multi-server Single-interface Queueing System with Random Connectivities" for which we did the stability analysis in Chapter 3 . We will show that for a symmetric system with i.i.d. Bernoulli arrival processes and i.i.d. connectivity processes, the Maximum Weighted Matching (MWM) policy not only is throughputoptimal but also is delay-optimal. Specifically, we will show that MWM minimizes, in stochastic ordering sense, a broad range of cost functions of the queue lengths including total queue occupancy (or equivalently minimizes the average queueing delay). We extend the model by considering imperfect services where it is assumed that the service of a scheduled packet fails randomly with a certain probability. We prove that the same policy is still optimal for the extended model. We also show that the results are still valid for some extended connectivity and arrival processes (e.g., Poisson arrival process).

\subsection{Research Motivation and Thesis Contributions}

The stability problems in wireless queueing systems have been studied in many papers $[1,3-13,16,17,19-21,23-27,29-33,35-39,44-61]$. However, in most of the research conducted in this area, the goal was to determine the optimal resource allocation policy in the studied models $[1,4,5,10,16,17,19-21,23-27,29-33,35,36,38,39,56$, 61]. The explicit characterization of the stability region in wireless systems has been studied in a small number of papers $[3,50-55,57-60]$. As we discussed before, the stability region is useful in solving utility optimization problems and also performance evaluation of the network control schemes. Thus, in the first part of our analysis (presented in Chapters 3 and 4), our contribution is to characterize, in an explicit manner, the system stability regions of a series of multi-server queueing systems with time-varying channel conditions. We also derive some explicit upper bounds for the average queue occupancy of each system. The derived upper bounds are especially useful to predict the buffering requirements of each system and also to develop Service Level Agreement (SLA) in wireless data networks.

While various performance attributes including throughput, power consumption, network life time, etc. have been studied in many papers [1,3-18], average queueing 
delay has been considered far less in the literature. This is due to the inherent difficulty of delay-optimal scheduling problems in queueing systems with time varying channel conditions. Therefore, in the second part of our analysis (which is presented in Chapter 5), we consider delay-optimal server allocation in the multi-server, singleinterface queueing system for which we performed the stability analysis in the first part of the thesis. Our contribution in this part of the thesis is to prove that MWM policy which is shown to be a throughput-optimal policy for such a system, is also delay optimal for a symmetric system. We will incorporate graph theory analysis and stochastic ordering arguments to prove this result.

From the practical perspective, the multi-server wireless queueing models considered in this thesis are inspired from the practical wireless systems. That is why we call such queueing systems "wireless queueing systems". In fact, the randomness of the connectivities between the queues and the servers in the studied systems is modeling the time variability of the wireless channels in realistic wireless systems. The system studied in Section 3.1 of Chapter 3 can be applied for modeling the transmitter allocation problem in a satellite node [62]. The multi-server systems studied in Sections 3.2 and 3.3 of Chapter 3 and in Chapters 4 and 5 can be applied in modeling the dynamic resource allocation in wireless access networks (See $[3,5,11-15,18,62-70])$.

In summary, the contributions of each chapter are summarized as follows:

- In Chapter 3, we focus on the stability analysis of multi-server queueing systems with random connectivities. Specifically, we consider three multi-server queueing systems which are applied to model the resource allocation problem in wireless communication networks. For each system, we will elaborate on the characterization of the stability region by use of a set of linear inequalities. The first system is a multi-interface queueing system with a randomly connected server bank which has been used to study the transmitter allocation problem in a satellite node [62]. The second system is a multi-queue multi-server (MQMS) queueing system with random connectivities which has been applied to model sub-carrier allocation problem in OFDMA (Orthogonal Frequency Division Multiple Access) systems [11-13,63,65]. Finally, the third system is a multi-server single-interface queueing system with random connectivities which has been used to study the channel allocation problem in wireless access networks [5]. For each system, we derive the necessary and sufficient conditions for the stability of the system for general packet arrival processes with finite 
first and second moments (Lemmas 3.2,3.3,3.5,3.6,3.9 and 3.10). It is shown (in Theorems 3.1, 3.2 and 3.3) that for stationary arrival processes the necessary and sufficient conditions define the stability region of the system. The stability region in each case is characterized by a finite set of linear inequalities on the arrival rates. In fact, the stability region is the intersection of a set of finite half-spaces in the non-negative orthant with binary normal vectors. We further obtain an upper bound for the average queue occupancy for each system. Using simulations, we will evaluate the obtained occupancy bound and also compare (in terms of average queue occupancy and stability region) the performance of the throughput-optimal policy for each system with that of a randomized policy.

- In Chapter 4, we extend the results of Chapter 3 for MQMS system to more general channel distributions. In this chapter, the stability region of multi-queue multi-server (MQMS) queueing system with stationary channel and arrival processes is characterized. Towards this end, the necessary and sufficient conditions for the stability of the system are derived for general arrival processes with finite first and second moments. For stationary arrival processes, these conditions establish the network stability region of the system. The main contribution in this chapter is to characterize the stability region as a convex polytope specified by a finite set of linear inequalities that can be numerically tabulated and used to solve network utility optimization problems (Refer to Lemmas 4.2 to 4.6 and Theorems 4.1 and 4.2). Furthermore, an upper bound for the average queueing delay of Maximum Weight (MW) policy [13] which is a throughput-optimal policy for MQMS systems is derived. Finally, the stability of fluid model MQMS systems is studied for which using the same approach as what we use in the packetized system, a linear algebraic representation of the stability region is characterized. It will be shown that the stability region in this case is characterized by an infinite number of linear inequalities. However, by an example it is concluded that depending on the channel distribution and the dimension of the system, the stability region may be characterized by a limited number of non-linear inequalities instead of infinite number of linear inequalities.

- In Chapter 5, we investigate the problem of delay-optimal server assignment in the multi-server single-interface queueing system with random connectivities for which we did the stability analysis in Section 3.3 of Chapter 3. For this system, 
it has been previously proven that Maximum Weighted Matching (MWM) is a throughput-optimal server assignment policy [4,5]. In Chapter 5, we show that the same policy is delay-optimal for a symmetric system with i.i.d. Bernoulli packet arrivals and connectivity processes (see Lemmas 5.1, 5.2, 5.3 and Theorem 5.1). More specifically, we show that MWM minimizes, in stochastic ordering sense, a broad range of cost functions of the queue lengths including total queue occupancy (or equivalently minimizes the average queueing delay). Then, we extend the model by considering imperfect services where it is assumed that the service of a scheduled packet fails randomly with a certain probability. We prove that the same policy is still optimal for the extended model. We also show that the results are still valid for some extended connectivity and arrival processes (e.g., Poisson arrival process).

\subsubsection{Contributions to the Literature}

The contributions to the literature resulted from this thesis are listed below:

- H. Halabian, I. Lambadaris, C.-H. Lung, "Optimal Server Assignment in Multi-Server Queueing Systems with Random Connectivities " submitted to IEEE/ACM Transactions on Networking.

- H. Halabian, I. Lambadaris, C.-H. Lung, "Explicit Characterization of Stability Region for Stationary Multi-Queue Multi-Server Systems " submitted to Transactions on Automatic Control.

- H. Halabian, I. Lambadaris, and C.-H. Lung, "Stability Analysis of Randomly Connected Multi-server Queueing Systems", submitted to Stochastic Models.

- H. Halabian, R. Changiz, F. Yu, I. Lambadaris, and H. Tang, "Optimal reliable relay selection in multiuser cooperative relaying networks," ACM/Springer Wireless Networks, vol. 18, pp. 591-603, August 2012.

- H. Halabian, I. Lambadaris, C.-H. Lung, "Optimal Server Assignment in Multiserver Parallel Queueing Systems with Random Connectivities and Random Service Failures" in Proc. IEEE International Conference on Communications (ICC 2012), Ottawa, ON, Canada, 2012. 
- H. Halabian, I. Lambadaris, C.-H. Lung, "Delay Optimal Server Assignment to Symmetric Parallel Queues with Random Connectivities" in Proc. 50th IEEE Conference on Decision and Control and European Control Conference (CDCECC 2011), Orlando, FL, USA, 2011.

- H. Halabian, I. Lambadaris, C.-H. Lung, "On the Stability Region of MultiQueue Multi-Server Queueing Systems with Stationary Channel Distribution" in Proc. IEEE International Symposium on Information Theory (ISIT 2011), Saint Petersburg, Russia, August 2011.

- H. Halabian, I. Lambadaris, C.-H. Lung, and A. Srinivasan, "Dynamic Channel and Interface Management in Multi-channel Multi-interface Wireless Access Networks", in Proc. IEEE GLOBECOM'10, Miami, FL, USA, Dec. 2010.

- H. Halabian, I. Lambadaris, C.-H. Lung, and A. Srinivasan, "ThroughputOptimal Relay Selection in Multiuser Cooperative Relaying Networks", in Proc. IEEE MILCOM'10, San Jose, CA, USA, Nov. 2010.

- H. Halabian, I. Lambadaris, C.-H. Lung, "Network Capacity Region of MultiQueue Multi-Server Queueing System with Time Varying Connectivities" in Proc. IEEE International Symposium on Information Theory (ISIT 2010), Austin, TX, USA, Jun. 2010.

\subsection{Notation}

In this section, the basic notation often used throughout the thesis is introduced. Additional notation will be introduced when necessary. All the vectors are considered to be row vectors. By $\underline{1}_{K}\left(\underline{0}_{K}\right)$, we denote a row vector of size $K$ whose elements are all identically equal to " 1 " (" 0 "). The time average of a function $f(t)$ is denoted by $\overline{f(t)}$, i.e., $\overline{f(t)}=\lim _{t \rightarrow \infty} \frac{1}{t} \sum_{\tau=1}^{t} f(\tau)$. The operator " $\circledast$ " is used for entry-wise multiplication of two matrices. The expectation of random processes (or random variables) is denoted by $E[\cdot]$. The cardinality of a set is denoted by $|\cdot|$. The operator for inner product of two vectors is $\langle\cdot, \cdot\rangle$. The boundary of a set is represented by bound $(\cdot)$, the closure of a set is denoted by $C l(\cdot)$ and the convex hull of a set is denoted by conv.hull $\{\cdot\}$. Function $(\cdot)^{+}$returns the term inside the brackets if it is non-negative and zero otherwise. For any vector $\alpha=\left(\alpha_{1}, \alpha_{2}, \ldots, \alpha_{N}\right)$ and a non-empty ordered 
(sub)set of indices $\mathcal{U}=\left\{u_{1}, u_{2}, \ldots, u_{|\mathcal{U}|}\right\} \subseteq\{1,2, \ldots, N\}$ and $u_{1}<u_{2}<\ldots<u_{|\mathcal{U}|}$, we define $\alpha_{\mathcal{U}}=\left(\alpha_{u_{1}}, \alpha_{u_{2}}, \ldots, \alpha_{u_{\mid \mathcal{u}}}\right)$.

\subsection{Thesis Organization}

The rest of the thesis is organized as follows.

- Chapter 2: This chapter titled "Background and Related Work", provides the preliminary definitions and required background for the contents of the subsequent chapters. Specifically, we review the fundamental concepts of network stability, stability region and throughput optimality in queueing systems. In this chapter, the back-pressure algorithm is also reviewed briefly as a throughputoptimal resource allocation policy for a general queueing network. Moreover, this chapter serves as a literature survey and review of related work in this area of research. References are provided for the interested readers to consult and obtain comprehensive discussions of each topic.

- Chapter 3: In this chapter titled "Stability Analysis of Randomly Connected Multi-server Queueing Systems" we study the stability analysis of three multiserver queueing systems with random connectivities. The stability analysis for each system is done in a separate section in which we describe the system assumptions in detail and then introduce the necessary and sufficient conditions for the stability of the system.

- Chapter 4: This chapter, titled "Explicit Characterization of Stability Region for Stationary Multi-Queue Multi-Server Systems", elaborates on the characterization of the stability region for stationary multi-queue multi-server systems which is the extended model of one of the systems considered in Chapter 3. For the aforementioned system, it will be shown that the stability region is a convex polytope for which a linear algebraic representation of the stability region will be determined.

- Chapter 5: In this chapter titled "Optimal Server Assignment in Symmetric Multi-server Single-interface Queueing Systems with Random Connectivities" we prove that MWM policy is optimal, in stochastic ordering sense, in minimization of a class of cost functions of the queue lengths including the total 
queue occupancy. We also extend the results for more general packet arrival and connectivity processes.

- Chapter 6: This chapter summarizes the conclusions of the thesis and highlights the next steps for future work. 


\section{Chapter 2}

\section{Background and Related Work}

In this chapter, the thesis research background and related work are provided by an overview on the definition of stability, stability region, throughput optimality and back-pressure algorithm. More specifically, in Section 2.1, the definition of strong stability is reviewed which is one of the general definitions of stability in queueing systems. Based on this definition, the notions of stability region and throughput optimality are introduced in Section 2.2. The stability region of a general queueing network is then characterized implicitly in Section 2.2.2 (See Theorem 2.1). Then in Section 2.2.3, the concept of back-pressure algorithm is explicated. This algorithm was introduced in the literature before and proven to be throughput-optimal (i.e., maximizes the stable throughput region of the system) for a general queueing system. The originality of the back-pressure algorithm comes from the fact that it acts without the need to know the stability region in advance. Note that the back-pressure algorithm guarantees the stability of the network when the arrival rate vector is inside the system stability region. However, when the input traffic rates are outside the stability region, we need to employ a flow control scheme in the transport layer. The flow control scheme controls the admitted traffic rates into the system so that the stability of the system is guaranteed while optimizing a level of fairness among the admitted traffic rates. Therefore, the transport layer has to perform optimal rate control by solving network utility optimization problems. By having an explicit characterization of the stability region, network utility optimization problems can be solved offline and with less buffering (queueing delay) overhead. Moreover, an explicit characterization of the stability region can be considered as a tool for performance evaluation of the proposed network control policies $[40,41]$. The applications of explicit characterization of stability region and the general form of network utility optimization problems 
are summarized in Section 2.3. As it was stated in the previous chapter, in the first part of this thesis we focus on the stability analysis of a series of multi-server wireless queueing systems. We will review the related work in this area in Section 2.4. In Section 2.5, we will elaborate on the notion of delay-optimal server allocation in wireless queueing systems and provide the necessary background and related work in this regard.

\subsection{Strong Stability Definition}

We start with introducing the definition of stability in queueing networks. In this thesis, we accept the definition of stability used in $[1,16]$ for our analysis and all of our results are based on this definition. Consider a time-slotted, single-queue, singleserver system with an arrival process $A(t)$ and service process $\mu(t)$ (See Figure 2.1). It is assumed that the arrivals are added to the system at the end of each time slot. It can be readily observed that the queue length process $X(t)$ evolves in time according to the following recursion.

$$
X(t)=(X(t-1)-\mu(t))^{+}+A(t)
$$

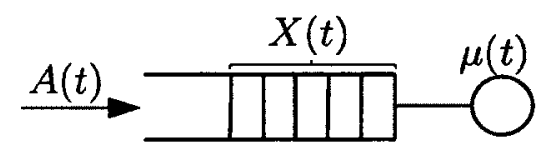

Figure 2.1: Single-queue single-server system

Definition 2.1 A queue evolving in time according to Eq. (2.1) is said to be strongly stable [1] if

$$
\limsup _{t \rightarrow \infty} \frac{1}{t} \sum_{\tau=0}^{t-1} E[X(\tau)]<\infty
$$

Definition 2.2 A queueing network is said to be strongly stable [1] if all the queues in the system are strongly stable. Specifically, for a network consisting of $N$ queues, the system is strongly stable if the time averaged expected aggregated backlog in the 
network is bounded, i.e.,

$$
\limsup _{t \rightarrow \infty} \frac{1}{t} \sum_{\tau=0}^{t-1} \sum_{n=1}^{N} E\left[X_{n}(\tau)\right]<\infty .
$$

In this thesis, the definition of strong stability is employed through all the stability analysis and in what follows the terms "stability" and "strong stability" are used interchangeably. In $[1,16]$, it was proved that if a queue is strongly stable and if for all $t$ either $E[A(t)] \leq A$ or $E[\mu(t)-A(t)] \leq D$ where $A$ and $D$ are finite given non-negative constants, then

$$
\lim _{t \rightarrow \infty} \frac{1}{t} E[X(t)]=0
$$

This property of stable queues is used in the proofs of the results in Chapters 3 and 4.

\subsection{Stability Region and Throughput-Optimal Server Allocation Policy}

Consider the discrete-time, single-queue, single-server system of Definition 2.1 with stationary packet arrival process $\{A(t)\}_{t=1}^{\infty}$ with rate $\lambda$, i.e., $E[A(t)]=\lambda$ for all $t$. We assume that the arrival random variables $A(t)$ have finite second moments, i.e., $E\left[A^{2}(t)\right]<\infty$. We also assume that the service to this queue is provided by a single server which is connected to the queue by probability $p$ (See Figure 2.2).

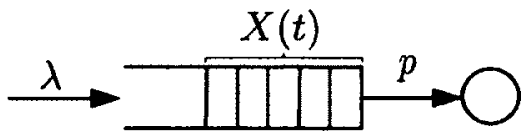

Figure 2.2: Single-queue, single-server system with random connectivity

During each time slot, the server can serve one packet from the queue if it is connected to the queue and the queue is not empty. We can think of $p$ as the service rate provided to the queue. Such a system can be used to model a single-queue, single-server wireless queueing system in which the traffic arrival rate is $\lambda$ and the queue (which is modeling a wireless user) is connected to the server (which can be 
used to model a communication sub-channel) with probability $p$. Using Lemma 3.6 in [1], we can show that the necessary and sufficient conditions for the stability of this system are the following:

- Necessary Condition: $\lambda \leq p$.

- Sufficient Condition: $\lambda<p$.

We define the closure of the set of arrival rates for which the system is stable as the system stability region. According to the necessary and sufficient conditions for the stability of the system, the stability region for the single-queue, single-server system is determined by $\lambda \leq p$.

Now consider a single-server system but with two queues similar to the two-queue, single-server system mentioned in Chapter 1 . It is assumed that the arrival processes to the queues are denoted by $\left\{A_{1}(t)\right\}_{t=1}^{\infty},\left\{A_{2}(t)\right\}_{t=1}^{\infty}$. These processes are assumed to be stationary with rates $\lambda_{1}$ and $\lambda_{2}$, respectively. We also assume that the queues 1 and 2 are connected to the server with probabilities $p_{1}$ and $p_{2}$, respectively. Note that the server can serve at most one packet from just one queue at each time slot. Therefore, in this case a server allocation policy must be applied to decide about the allocation of the server to the queues. Figure 2.3 depicts the two-queue single-server system described above.

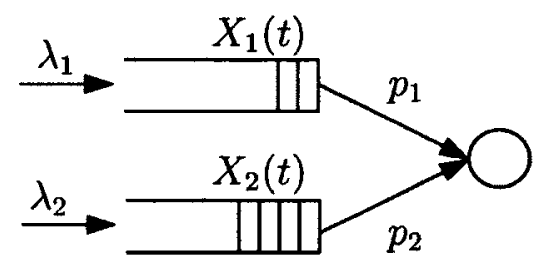

Figure 2.3: Two-queue single-server system with random connectivities

The characterization of stability region for this system is not as straight forward as the single-queue single-server system since the stability of the system is dependent to the applied server allocation policy. We define the indicator variable $I(t)$ to be 1 if queue 1 is served at time slot $t$ and zero if queue 2 is served at time slot $t$. Therefore, any server allocation policy $\pi$ can be determined by the set of all the indicator variables $I^{(\pi)}(t), t=1,2, \ldots$, i.e., $\pi=\left\{I^{(\pi)}(t)\right\}_{t=1}^{\infty}$ in which $I^{(\pi)}(t)$ is the indicator variable of policy $\pi$ at time slot $t$. For a policy $\pi$ we define the closure 
of the set of all the arrival rate vectors $\left(\lambda_{1}, \lambda_{2}\right)$ for which policy $\pi$ can stabilize the system as the stability region of policy $\pi$.

The stability region of different policies might be different. One might imagine that the stability regions of all the work-conserving server allocation policies ${ }^{1}$ are the same. We will see that this is not the case and not all work-conserving policies have the same stability region. For example, in a system with two connected queues with queue lengths one and ten packets respectively serving the shorter queue can potentially create a higher probability that this queue is empty in the near future. This results in loosing the use of multi-user diversity in future time slots and creating potential service loss and consequently potential instability [1]. In the following example, we will evaluate the stability regions of three sample server allocation policies studied in literature $[1,71,72]$.

Example: Borst Algorithm: The Borst algorithm was proposed in [72] as a channel aware scheduling strategy in wireless data networks. This algorithm chooses to serve the non-empty queue with the largest $\mu_{n}(t) / E\left[\mu_{n}(t)\right]$ where $\mu_{n}(t)$ is the rate offered to queue $n$ if this queue is served and $E\left[\mu_{n}(t)\right]$ is the expected value of service rate provided to this queue. For the described two-queue single-server system we have $\mu_{n}(t)=1$ if queue $n$ is connected and is zero if it is disconnected at time slot $t$. We also have $E\left[\mu_{1}(t)\right]=p_{1}$ and $E\left[\mu_{2}(t)\right]=p_{2}$. Therefore, the Borst algorithm for this system is simplified to give the priority of service to the non-empty queue with the largest $1 / p_{n}$ factor (i.e., to the non-empty queue with the minimum probability of connectivity). Assuming that $p_{1}<p_{2}$, it is not hard to show that the stability region of the Borst algorithm is determined by the following inequalities $[1,72]$.

$$
\lambda_{1} \leq p_{1}, \quad \lambda_{2}+p_{2} \lambda_{1} \leq p_{2}
$$

Example: $\operatorname{Max} \mu_{n}(t) / r_{n}(t)$ Algorithm: This policy was proposed in [71] and serves the non-empty queue with the largest $\mu_{n}(t) / r_{n}(t)$ value in which $\mu_{n}(t)$ is the service offered to queue $n$ at time slot $t$ (which is 1 when the queue is connected and is 0 otherwise) and $r_{n}(t)$ is the empirical service rate received by queue $n$. The difference between this algorithm and the Borst algorithm is that in this policy, $\mu_{n}(t)$ is weighted by the actual service rate delivered to queue $n$ while in the Borst algorithm it is weighted by the service rate offered to queue $n$. Note that if the system is stable, in

\footnotetext{
${ }^{1}$ We say a policy is work conserving if it does not waste the service by idling the server while there exists non-empty connected queues
} 
the long term operation of the system $\left(r_{1}(t), r_{2}(t)\right)$ converges to $\left(\lambda_{1}, \lambda_{2}\right)$. Therefore, this policy gives strict priority to queue 2 if $\lambda_{1}>\lambda_{2}$ and gives strict priority to queue 1 if $\lambda_{1}<\lambda_{2}$. In summary, the stability region of this policy is determined by the following inequalities $[1,71]$.

$$
\begin{array}{ll}
\text { - if } \lambda_{1}>\lambda_{2}: & \lambda_{2} \leq p_{2}, \quad \lambda_{1}+p_{1} \lambda_{2} \leq p_{1} \\
\text { - if } \lambda_{1}<\lambda_{2}: & \lambda_{1} \leq p_{1}, \quad \lambda_{2}+p_{2} \lambda_{1} \leq p_{2}
\end{array}
$$

Example: Longest Connected Queue ( $L C Q$ ): This policy serves the longest connected queue at each time slot. The stability region for this policy is determined by the following inequalities [3].

$$
\begin{gathered}
\lambda_{1} \leq p_{1} \\
\lambda_{2} \leq p_{2} \\
\lambda_{1}+\lambda_{2} \leq p_{1}+p_{2}-p_{1} p_{2}
\end{gathered}
$$

These inequalities can be interpreted intuitively by considering the following facts: Each of queues 1 and 2 cannot get service with rates more than $p_{1}$ and $p_{2}$, respectively. Moreover, the server will not be idle if it is connected to at least one non-empty queue.

The stability regions of the policies described above are depicted in Figure 2.4 for $p_{1}=0.6$ and $p_{2}=0.7$. As we can see from this figure, the stability region of the Borst and $\operatorname{Max} \mu_{n} / r_{n}$ algorithms are subsets of the stability region of LCQ policy, i.e., LCQ policy can stably serve more arrival rate vectors.

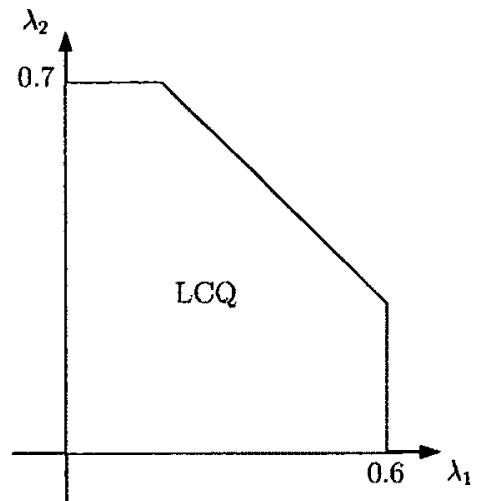

(a) LCQ

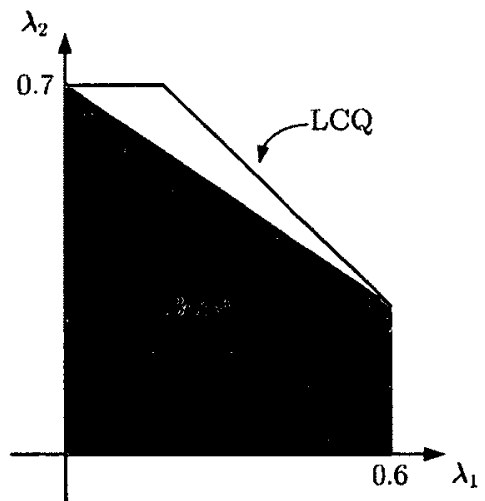

(b) Borst

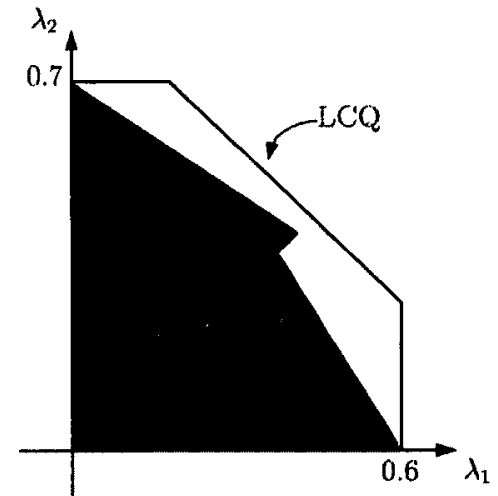

(c) $\operatorname{Max} \mu_{n}(t) / r_{n}(t)$

Figure 2.4: Stability regions of LCQ, Borst and $\operatorname{Max} \mu_{n}(t) / r_{n}(t)$ policies 
We define the system stability region as the closure of the set of all the arrival rate vectors for which there exists a server allocation policy that can stabilize the system $[1,4,5,9,16,17,24,38]$. The system stability region should be differentiated from the stability region of a policy. The stability region of a policy is the collection of all the traffic rate vectors that are stably supported by a specific policy. However, the system stability region is the union of the stability regions of all the possible server allocation policies. Thus, there is no server allocation policy that can stabilize the system for the input arrival rates outside the system stability region. Clearly the first attribute for evaluating the performance of a given policy is by examining its stability region. The larger the stability region the better the performance of the policy since it can stably support more traffic arrival rates. A server allocation policy whose stability region coincides with the system stability region is called throughputoptimal $[1,4,5,12,13,17,38,65]$.

For the two-queue, single-server system, in $[1,3,16]$ it has been shown that the system stability region is characterized by the set of inequalities indicated in (2.6). Therefore, LCQ policy is a throughput-optimal server allocation policy for the two-queue, single-server system. Note that the analysis for the two-queue, single-server system has been generalized in [3] for a multi-queue single-server system with $N$ queues, with arrival rate vector $\left(\lambda_{1}, \lambda_{2}, \ldots, \lambda_{N}\right)$ and connectivity probabilities $p_{1}, p_{2}, \ldots, p_{N}$. For such a system, it has been shown that the stability region is characterized by the following set of inequalities.

$$
\sum_{n \in Q} \lambda_{n} \leq 1-\prod_{n \in Q}\left(1-p_{n}\right) \quad \forall Q \subseteq\{1,2, \ldots, N\}
$$

Furthermore, it was shown that LCQ policy which serves the longest connected queue at each time slot is throughput-optimal.

The discussion regarding the system stability region and throughput optimality can be easily extended to a general queueing network with arbitrary topology modeling. In the following, we will describe a general queueing network for which we will repeat the definitions of system stability region and throughput-optimal policy. Later, we will introduce back-pressure algorithm as a throughput-optimal resource allocation algorithm for a general queueing network. 


\subsubsection{Model Description-The General Queueing Network Case}

Consider a time-slotted, wireless network with $N$ wireless nodes. Assume that the data packets destined to node $c \in\{1,2, \ldots, N\}$ is labeled as commodity $c$ data. Each node $a$ in the network is keeping the data packets of each commodity $c$ in a separate queue $Q_{a}^{(c)}$. Assume that the length of $Q_{a}^{(c)}$ at the end of time slot $t$ is denoted by $X_{a}^{(c)}(t)$. Each node is fed exogenously by arrival processes $\left\{A_{n}^{(c)}(t)\right\}_{t=1}^{\infty}$ (this is a general model and for the nodes without exogenous arrivals of commodity $c$ we can put $\left.A_{n}^{(c)}(t)=0, \forall t\right)$. In fact, $A_{n}^{(c)}(t)$ represents the amount of traffic (packets) entered to queue $Q_{a}^{(c)}$ exogenously at time slot $t$. It is assumed that during a time slot, departures occur first, then the arrivals of that time slot are added to the queues at the end of the time slot and the queue lengths are updated. We assume that the arrival processes are stationary with finite second moments and with rate $\lambda_{a}^{(c)}$. By putting all the arrival rates in a matrix we can form the arrival rate matrix $\lambda=\left(\lambda_{n}^{(c)}\right)$.

We assume that the capacity of the link between nodes $a$ and $b$ is denoted by $C_{a, b}(t)$ packets/time slot. In other words, link $(a, b)$ can accommodate $C_{a, b}(t)$ successful packet transmissions from node $a$ to node $b$ at time slot $t$. By putting all the channel capacities $C_{a, b}(t)$ for all the communication links together we can form the channel capacity matrix $C(t)=\left(C_{a, b}(t), a, b=1,2, \ldots, N\right) . C(t)$ is a function of the topology state of the system denoted by $S(t)$ and the control decision (resource allocation) vector $I(t)$ and therefore can be written as $C(S(t), I(t))$. S(t) not only captures the effects of the the physical location of the nodes but also captures the effects of other physical layer parameters of the system such as applied modulation/coding scheme, fading, noise, interference, etc. It is assumed that the topology state process $S(t)$ takes values from a finite (but arbitrarily large) sample space $\mathcal{S}$. We also assume that the topology state process is ergodic and has stationary probabilities denoted by $P(s)$, i.e., $P(s)=\operatorname{Pr}(S(t)=s), \forall s \in \mathcal{S} . I(t)$ is the vector of all the resource allocation variables (e.g., bandwidth, power, etc.) in the system. The problem of optimal resource allocation in this system is to find an appropriate control decision vector at each time slot according to which the network resources are allocated to the wireless nodes for data transmissions. For example in the two-queue, single-server system, the topology state variables at time slot $t$ are the connectivity variables of the two queues, i.e., $S(t)=\left(C_{1}(t), C_{2}(t)\right)$ where $C_{n}(t), n=1,2$ is the connectivity variable associated to queue $n$ at time slot $t$ which is 1 if the queue is connected to the server at time slot $t$ and zero otherwise. $I(t)$ for the two-queue, single-server system 
is defined as the indicator variable $I(t)$ which was defined to be 1 if the scheduling policy decides to allocate the server to queue 1 and zero if the server is allocated to queue 2.

The decision vector $I(t)$ is usually restricted to satisfy some constraints depending on the assumptions regarding the underlying network ${ }^{2}$. In general, we assume that the decision vector space is dependent on the topology state. The decision vector space when the system is in state $s$ is denoted by $\mathcal{I}_{s}$. Thus, $\mathcal{I}_{s}$ denotes the set of valid decision vectors when the system is in topology state $s$.

A resource allocation policy $\pi$ is said to stabilize the system if by applying policy $\pi$ the system is strongly stable. Let $\Lambda_{\pi}$ represent the closure of the set of arrival rate matrices for which $\pi$ can stabilize the system. $\Lambda_{\pi}$ is called the stability region of policy $\pi$. Now, consider the closure of the union of the stability regions of all the possible resource allocation policies and let us denote it by $\Lambda$, i.e., $\Lambda=C l\left(\bigcup_{\pi} \Lambda_{\pi}\right)$. $\Lambda$ is called system stability region (also called network layer capacity region in the literature $[1,9,14-17,24,38,61])$. Therefore, network stability region is the closure of the set of all the arrival rate matrices for which there exists a resource allocation policy that can stabilize the system. Note that the network stability region is independent of resource allocation policy and is in fact a specific characteristic of any network.

A resource allocation policy $\pi^{*}$ is called throughput-optimal if it can stabilize the system for all the arrival rate matrices strictly inside the network stability region. Therefore, $\pi^{*}$ is throughput-optimal if its stability region coincides with the network stability region. Note that an explicit characterization of the system stability region by a set of inequalities on the arrival rates (like what was used in (2.6) for the two-queue single-server system) is not always an easy task. However, based on the definition of the stability region provided above, the system stability region can be characterized implicitly in the following section.

\subsubsection{Implicit Characterization of the System Stability Region}

We start with introducing the region of achievable transmission rates over the communication links of the general system described in the previous subsection. This is done by considering two important facts:

\footnotetext{
${ }^{2}$ For example, in the two-queue, single-server system, the server is constrained to serve at most one queue per time slot. These modeling constraints usually are dictated by the system designer according to the limitations of the realistic wireless systems.
} 
- Because of time variability of the topology state and therefore channel capacities, the achievable transmission rates over each link $(a, b)$ is averaged over all the possible topology states.

- The achievable transmission rates at time slot $t$ when the system is in state $s \in \mathcal{S}$ are not fixed and depend on the resource allocation decisions $I(t) \in \mathcal{I}_{s}$ applied at time slot $t$.

By considering these facts, the set of achievable rates is characterized by the following matrix $[1,16]$.

$$
\Gamma=\sum_{s \in \mathcal{S}} P(s) \text { conv.hull }\left\{C(s, I) \mid I \in \mathcal{I}_{s}\right\}
$$

where scalar addition and multiplication of sets is used and where conv.hull $\{\cdot\}$ is the convex hull of a set. Note that convex hull of a set $A=\left\{a_{1}, a_{2}, \ldots, a_{|A|}\right\}$ is defined as conv.hull $\{A\}=\left\{p_{1} a_{1}+p_{2} a_{2}+\ldots+p_{|A|} a_{|A|} \mid a_{i} \in A, p_{1}+p_{2}+\ldots+p_{|A|}=1\right\}$. To intuitively explain why $\Gamma$ in (2.8) contains all the achievable transmission rates, we note the following fact. Suppose that the system is in state $s$. This happens with probability $P(s)$. When the system is in state $s$, we can apply $\left|\mathcal{I}_{s}\right|$ distinct decision vectors. Each decision vector $I \in \mathcal{I}_{\boldsymbol{s}}$ provides us a deterministic transmission rate matrix $C(s, I)$. Therefore, if we deterministically associate a fixed decision vector for each topology state, we obtain a deterministic policy which provides us a fixed transmission rate. However, when the system is in state $s$ we may also apply a randomized policy in which each decision vector $I \in \mathcal{I}_{s}$ is applied with some probability. Thus, by using a randomized policy all the transmission rate matrices in $\Gamma$ are achievable. It is noticeable that the randomized policy will cover all the possible resource allocations and therefore it can achieve all the possible transmission rate matrices. Therefore, any transmission rate matrix $G \in \Gamma$ is achievable by applying an appropriate randomized resource allocation policy. For more information regarding the definition and properties of set $\Gamma$, the reader is invited to consult with [1].

By having set $\Gamma$ given in (2.8), we can characterize the system stability region by the following theorem introduced in $[1,16,38]$.

Theorem 2.1 (System Stability Region for a General Queueing Network) The Stability region of a network is given by the set $\Lambda$ consisting of all the arrival rate matrices $\lambda=\left(\lambda_{n}^{(c)}\right)$ such that there exists transmission rate matrix $G \in C l\{\Gamma\}$ together with 
multi-commodity flow variables $f_{a b}^{(c)}$ satisfying the following constraints:

- Flow Efficiency Constraints: $f_{a b}^{(c)} \geq 0, f_{c b}^{(c)}=0$ for all $a, b, c$

- Flow Conservation Constraints: $\lambda_{i}^{(c)} \leq \sum_{b} f_{i b}^{(c)}-\sum_{a} f_{a i}^{(c)}$ for all $i, c, i \neq c$

- Link Constraints: $\sum_{c} f_{a b}^{(c)} \leq G_{a b}$, for all $a, b$

Note that node $c$ is the destination of commodity $c$ data packets.

The first condition in Theorem 2.1 states that the flow variables $f_{a b}^{(c)}$ are nonnegative. This means that no node transmit data packets to itself. Moreover, it states that $f_{c b}^{(c)}=0$ which means that no destination node $c$ will re-inject the delivered data packets into the system. The next condition states that the total amount of commodity $c$ data packet flows into a given node is equal to the total amount of commodity $c$ data packet flows out of that node. This is called flow conservation constraint which means that data packets of a given commodity are never dropped in the intermediate nodes. The last condition states that the maximum amount of traffic out of a given node for all the commodities cannot exceed the capacity of the outgoing link.

To clarify the definition of $\Gamma$ and how Theorem 2.1 characterizes the system stability region, we will again consider the two-queue single-server system for which we characterize $\Gamma$ and the stability region using Theorem 2.1. In this system, we have $\mathcal{S}=\{(0,0),(0,1),(1,0),(1,1)\}$ which states that the links from the queues to the server are either connected or disconnected. The probability of these states are given by $\operatorname{Pr}(S(t)=(0,0))=\left(1-p_{1}\right)\left(1-p_{2}\right), \operatorname{Pr}(S(t)=(0,1))=\left(1-p_{1}\right) p_{2}$, $\operatorname{Pr}(S(t)=(1,0))=p_{1}\left(1-p_{2}\right)$ and $\operatorname{Pr}(S(t)=(1,1))=p_{1} p_{2}$. Recall the definition of decision variable for this system as

$$
I(t)= \begin{cases}1, & \text { if the server is allocated to queue } 1 \\ 0, & \text { if the server is allocated to queue } 2\end{cases}
$$

the transmission rate matrix in this case would be a vector of size two denoted by $C(S(t), I(t))=\left(C_{1}(S(t), I(t)), C_{2}(S(t), I(t))\right)$. Using $(2.8)$, we can characterize $\Gamma$ by

$$
\begin{aligned}
\Gamma= & \left(1-p_{1}\right)\left(1-p_{2}\right)\{(0,0)\}+\left(1-p_{1}\right) p_{2}\{(0,1)\} \\
& +p_{1}\left(1-p_{2}\right)\{(1,0)\}+p_{1} p_{2} \text { conv.hull }\{(1,0),(0,1)\}
\end{aligned}
$$


Using Theorem 2.1, for this simple example it can be verified that the stability region for two-queue single-server system is given by the set of all the non-negative rate vectors $\left(\lambda_{1}, \lambda_{2}\right)$ satisfying the inequalities given in (2.6).

Characterizing the stability region in Theorem 2.1 is an implicit form of characterization of the stability region. In fact, explicit characterization of the stability region in terms of a set of inequalities on the arrival rates (like in (2.6)) for each specific queueing system requires further analysis. The analysis for the two-queue single-server system was quite straight forward. However, by increasing the number of queues and the number of servers and the state space for the channel capacity of a link (from binary ON-OFF channels to $M$-ary channels) the analysis gets much more complicated. In the first part of this thesis, we will study this problem for some multi-server queueing systems with time varying channel conditions which are applied in modeling the multi-channel multi-user wireless access networks. For each system, we obtain an explicit characterization of the stability region using a set of linear inequalities.

\subsubsection{Back-pressure Algorithm}

Although the explicit characterization of the stability region is not an easy task to perform for a general queueing system, the throughput-optimal policy has been already determined for a general queueing system in $[1,4,9,16,17,23,24,38,39,61]$. In this section, the back-pressure algorithm is studied which has been shown to be a throughput-optimal joint resource allocation/routing scheme for a general queueing network $[1,4,16,17]$.

Consider the general queueing network described in subsection 2.2.1. For this system, we will determine the throughput-optimal resource allocation strategy by determining decision vector $I(t)$ and also the optimal routing of data packets in the network.

This algorithm consists of two major stages. In the first stage which is the resource allocation stage, the optimal control decision vector is determined as follows $[1,4,16$, 38]:

- For each link $(a, b)$, the optimal commodity is determined as

$$
c_{a, b}^{\star}(t)=\arg \max _{c}\left(X_{a}^{(c)}(t-1)-X_{b}^{(c)}(t-1)\right)
$$


- The corresponding optimal weight associated to link $(a, b)$ is defined as

$$
W_{a, b}^{\star}(t)=\left(X_{a}^{\left(c_{a, b}^{\star}(t)\right)}(t-1)-X_{b}^{\left(c_{a, b}^{\star}(t)\right)}(t-1)\right)^{+}
$$

- The optimal control decision variables are determined by

$$
I^{\star}(t)=\arg \max _{I(t) \in \mathcal{I}_{S(t)}} \sum_{a, b} W_{a, b}^{\star}(t) C_{a, b}(S(t), I(t))
$$

In the routing stage, the data packets of commodity $c_{a, b}^{\star}(t)$ are routed over link $(a, b)$ with rate $C_{a, b}\left(S(t), I^{\star}(t)\right)$.

As discussed earlier, it has been proven that back-pressure algorithm is throughput-optimal and stabilizes the system for all the arrival rate matrices strictly inside the stability region $[1,4,16,38]$. The capability of back-pressure algorithm to stabilize the network can be explained intuitively by considering the following points. For each time slot,

- The back-pressure algorithm calculates the maximum differential backlog for each communication link and uses this value as the weight of the link capacity.

- It then uses the non-negative weights and multiplies them by the link capacities and maximizes the total weighted capacity of the system. The solution to this maximization is the optimal resource allocation for that time slot. By doing so, the back-pressure algorithm tries to give more resources to the links with higher level of differential backlog which have good channel conditions. Therefore, this algorithm provides more service rate to the queues that are capable of serving a lot of packets (have good channel condition) while having a large amount of packets waiting for service. In fact, the back-pressure algorithm not only tries to maximize the link capacities in the system but also it tries to balance the queues to keep the system stable and prevent the queue lengths from growing to infinity.

- In the routing part, this algorithm will route the data packets of the commodity which has the maximum differential backlog. 


\subsection{Explicit Characterization of the Stability Region}

Note that back-pressure algorithm does not need to know the stability region to act. In fact, this is the novelty of this algorithm that acts just based on the available information about the topology state of the system (i.e., $S(t)$ ) and the queue length state (i.e., $X(t-1))$.

Although stability region is not needed to be known for the operation of backpressure algorithm, an explicit characterization of the stability region can be used as a tool for performance evaluation of network control policies. For example in $[1,16,17,41]$, the authors provided some upper bounds for the average occupancy of back-pressure algorithm. Those bounds are implicit bounds since they are defined based on the distance of the operating point of the system from the boundary of the stability region. For example, the authors in [41] proposed a delay bound for the Maximum Weight policy which is tight in heavy-traffic limit. However, the proposed bound is parametrized by the distance of the arrival rate point from the facets of the stability region polytope. Without having an explicit characterization of the stability region, deriving the values of those bounds is not an easy task. An explicit characterization of the stability region in terms of a set of linear inequalities (Linear algebraic characterization) is also useful for deriving explicit bounds on the flow throughput (which is a flow level performance measure) under balanced fairness [40].

An explicit characterization of the stability region can also be useful in solving network utility optimization problems. In the following, we will review the general form of network utility optimization problems briefly.

\subsubsection{Utility Optimization Problems}

Apart from the resource allocation problem (which is done in the physical and MAC layers of wireless networks), flow control plays a crucial role in ensuring the system stability while achieving a level of network fairness. Flow control strategies are applied when the input rates are outside the stability region. A flow control strategy must decide what fraction of the injected traffic to the system must be admitted to assure the stability of queues in the network layer while achieving the optimal network fairness among users.

A quantitative measure of fairness which is widely used in literature (e.g. $[1,2,16$, $23,28,34,35,38,39,73-77])$ is to define a set of utility functions $f_{n}(r)$ which illustrate 
the grade of satisfaction for each user $n$ while it transmits data traffic at rate $r$. Consider a general network (wired or wireless) with $N$ traffic sources. Suppose that $\lambda_{n}, n=1,2, \ldots, N$ is the traffic arrival rate of each source $n$. Let us denote the network stability region by $\Lambda$. The flow control strategy determines the admitted rates $r_{n}$ from each source $n$ by solving the following flow maximization problem.

$$
\begin{array}{cl}
\underset{r_{1}, r_{2}, \ldots, r_{N}}{\operatorname{Maximize}:} & \sum_{n=1}^{N} f_{n}\left(r_{n}\right) \\
\text { Subject to: } & r=\left(r_{1}, r_{2}, \ldots, r_{N}\right) \in \Lambda \\
& 0 \leq r_{n} \leq \lambda_{n} \quad \forall n=1,2, \ldots, N
\end{array}
$$

By solving the above maximization problem, each node adjusts its admitted traffic rate to the computed optimal point from (2.10). The functions $f_{n}\left(r_{n}\right)$ are assumed to be non-decreasing and concave. The choice of functions $f_{n}\left(r_{n}\right)$ depends on the desired fairness properties in the network. For example, choosing the utility function $f(r)=$ $\log (1+\beta r$ ) (for some large constant parameter $\beta>0$ ) will result in proportional fairness behaviour $[1,74]$.

Note that there have been proposed several dynamic flow control strategies in the literature which are able to solve such network optimization problems without having an explicit characterization of the stability region $[1,16,38,38,39,73,77]$. However, the cost for the closeness of the operating point of these strategies to the optimal point is to suffer larger queueing delay. By having the stability region characterized explicitly, we can solve these network optimization problems offline and make the wireless users to regulate their admission rates equal to the optimal point (for example by using a leaky bucket). This makes the queues to be smoothly fed by the flow controller and therefore congestion is avoided in the system. In other words, by having an explicit characterization of the stability region, network utility optimization problems can be solved offline and with less buffering (queueing delay) overhead. This fact is shown by a numerical example in Chapter 4 .

\subsection{Wireless Queueing Systems}

In realistic wireless systems, the quality of the wireless channel is changing over time due to different factors such as mobility of wireless entities, path loss, shadowing 
and fading phenomena $[78,79]$. Depending on the time scale in which the wireless channel is being studied, different approaches for the modeling of a wireless channel can be considered. For example the model applied when we are studying a wireless system in the physical layer is different from the model applied when we study the system in the network layer. In the physical layer we are dealing with problems in the transmission time scale of a communication symbol (or bit). However, in the network layer we study the system in the transmission time scale of a packet. In this thesis, our models do not reflect the physical layer issues such as modulation, coding, etc. We study the system from higher layers of the communication protocol stack, i.e., MAC, network and transport layers. We consider time slotted wireless queueing models with equal-length time slots and equal-length packets. The duration of a time slot is chosen such that during a time slot the quality of the wireless channel (which is usually measured by received SINR $^{3}$ ) would be fixed. This can be done by choosing the duration of a time slot with respect to the coherence time of the wireless system so that channels stay constant within each slot and vary across time slots.

Depending on the applied channel modeling and also the selected packet lengths, a single time slot may accommodate up to $M \in \mathbb{Z}_{+}$successfully packet transmissions. For $M=1$ we have the channels modeled as an ON-OFF channel in which during a time slot we may transmit at most one packet and depending on the channel condition this transmission may be successful or may be failed. In this case, we say that the channels are binary channels and we rename the term "channel" by the term "connectivity". This modeling is validated by assuming that the transmission of a wireless transmitter to a wireless receiver over a wireless channel is successful if the the received SINR exceeds a certain threshold. In the queueing systems studied in Chapters 3 and 5 the channels are assumed to be binary.

For $M>1$ the wireless channel can accommodate more than one packet transmission. In this case, usually there exist $M$ SINR threshold values such that when the received SINR exceeds the $m$ th threshold $(m=1,2, \ldots, M)$, this guarantees $m$ successful packet transmissions over the channel at that time slot. In this case, the channels are called to be $M$-ary channels. In Chapter 4 , we study the stability of a queueing system with $M$-ary channels.

\footnotetext{
${ }^{3}$ Signal to Interference plus Noise Ratio
} 


\subsubsection{Multi-server Wireless Queueing Systems}

In Section 2.2, we discussed about the stability region analysis of wireless queueing networks and to support our discussion we did the analysis for the example of a two-queue, single-server system. In this thesis, we focus on the stability analysis and optimal stochastic control of multi-server wireless queueing system in which the queues are competing to get service from a set of shared servers (instead of a single server). Such systems are used to model the resource allocation problem in the emerging wireless access networks and satellite networks $[3,5,11-15,62-67,80,81]$. Examples of such networks are OFDMA and CDMA wireless systems in which orthogonal resources (OFDM subcarriers and CDMA codes) must be allocated to multiple users. Another example is wireless relaying access networks where the relay stations are the resources that must be shared among wireless users [18]. In these networks, the wireless users can be modeled by the queues and the network resources may be modeled by the servers.

The two-queue, single-server system studied before is a special case of the multiqueue, single-server system studied in [3] where $N$ queues are sharing a single server and the connectivity of each queue to the server is varying across time slots. Now, consider a generalization of the multi-queue, single-server system in which we increase the number of servers from 1 to $K>1$ and put them all in a server bank. In this case, the connectivity of each queue to the server bank is varying over time slots. Note that if the queues are allowed to get service from as many servers as they want, the new system will be a trivial generalization of the multi-queue, single-server system. However, if we limit the queues to get service from at most $R$ servers during each time slot, we obtain a system which is not a trivial generalization of the multiqueue single-server system. In the case that the queues are constrained to get service from at most $R$ servers at each time slot, we say that the queues are equipped with $R$ service interfaces (radio interfaces). Therefore, we call the new system "multiinterface queueing system with a randomly connected server bank". This system with one interface per queue, i.e., $R=1$, has been used in [62] to model the transmitter allocation problem in a satellite node. We will study the stability properties of this system in Chapter 3.

We can further generalize the multi-queue single-server system by increasing the number of servers to $K$ but in this case we do not put them all in a server bank. 
Instead, we assume that each queue has a separate connectivity to each server. Therefore, in this system we have $N \times K$ connectivity variables which may be independent or not. There is no restriction for the number of servers that can serve each queue at each time slot. This is equivalent to assuming that each queue is equipped with $K$ interfaces. Such a system is called "multi-queue multi-server system with random connectivities" through the thesis. This system is used in $[11-13,63,65]$ to study subcarrier allocation problem in OFDMA access networks. We will study the stability properties of this system in Chapter 3 . We will also study the stability analysis of the same system with $M$-ary channels (rather than binary channels) in Chapter 4 .

Next, we study a queueing system similar to the multi-queue, multi-server system but with just one interface per queue. Hence, each queue can get service from at most one server during each time slot. This system is called "multi-server single-interface queueing system with random connectivities" whose stability analysis is studied in Chapter 3 of the thesis. We also study the server assignment problem in a symmetric version this system with i.i.d. Bernoulli packet arrival and connectivities in chapter 5 with the objective of minimizing the average queueing delay.

\subsubsection{Stability Analysis in Wireless Queueing Systems}

The stability problem in wireless queueing networks was mainly addressed in $[1,3$, $4,7,16,17,38]$. The notion of stability region of a queueing network was originally introduced by Tassiulas and Ephremides in the seminal work [4]. In [3], they also characterized the network stability region of multi-queue single-server systems with time varying connectivities. In $[1,16,17,23,24,38,61]$, the notion of network stability region of a wireless network was introduced for more general arrival and queue length processes. Furthermore, Lyapunov drift techniques were applied in $[1,5,6,10,16,17,23$, $24,38,61]$ to analyse the stability of the proposed policies for stochastic optimization problems in wireless networks.

The problem of server allocation in multi-queue multi-server (MQMS) systems with time varying connectivities was mainly addressed in [5, 12-14,63, 65]. In [13], Maximum Weight (MW) policy was proposed as a throughput-optimal server allocation policy for MQMS systems with stationary channel process. However, in [13] the conditions on the arrival traffic to guarantee the stability of MW were not explicitly mentioned. In [5], it was shown that for a multi-server single-interface queueing system with random connectivities (in which queues are restricted to attract at 
most one server at each time slot), Maximum Weighted Matching (MWM) policy is throughput-optimal. The authors also considered the effect of infrequent channel state measurements on the network stability region.

The resource allocation problem in multi-channel multi-interface networks was generally discussed in [80-83]. These papers address the resource allocation problem in a multi-hop wireless network. The authors in [80] study a system in which the channels are interfering with each other and the objective is to reduce the overall interference in the network. The references $[81,82]$ focus on channel scheduling in multi-channel single-interface multi-hop networks with limited information exchange. In [83], the authors proposed a joint congestion control, channel allocation and scheduling algorithm based on a specific model. The main goal of the proposed algorithm is to maximize a utility function of the injected traffic while stabilizing the queues in the network.

\subsection{Delay-Optimal Server Allocation in wireless Queueing systems}

As we discussed in Chapter 1, the main goal in the stochastic control of wireless networks is to distribute the shared network resources among multiple users such that certain stochastic performance attributes are optimized. While various performance attributes including the stable throughput region, power consumption, utility functions of the admitted traffic rates and network life time have been studied in many papers [1-37], average queueing delay has been considered far less in the literature $[3,42,43,62,63,65,84]$. This is due to the inherent difficulty of delay-optimal scheduling problems in queueing systems with time-varying channel conditions.

We discussed in Section 2.2.3 that the back-pressure algorithm is a throughputoptimal policy for a general queueing network. However, maximizing the stable throughput of the system does not translate to minimizing the average queueing delay. To clarify this fact, consider the following example.

Example: Consider again the two-queue, single-server system. For this system, we have seen that LCQ policy is throughput-optimal. Recall that LCQ serves the longest connected queue at each time slot. Now assume that we apply the same policy for this system but we update the queue lengths every four time slots, i.e., we update the queue lengths only at the end of the time slots $T=4 t, t=1,2, \ldots$. 
In [5], it has been proven that infrequent updating the queue lengths does not shrink the stable throughput region of the system. Therefore, both policies (i.e., LCQ with frequent and infrequent queue updates) maximize the stable throughput region of the system and are throughput-optimal. However, the second policy with infrequent queue length updates will suffer larger average queueing delay than LCQ. Figure 2.5 shows the average queue occupancy for the two policies versus the arrival rate $\lambda$ (It is assumed that $\lambda_{1}=\lambda_{2}=\lambda$ ) where $p_{1}=0.4$ and $p_{2}=0.8$. It is apparent that both systems have the same stability region but the average queue occupancy of LCQ is less than the other policy.

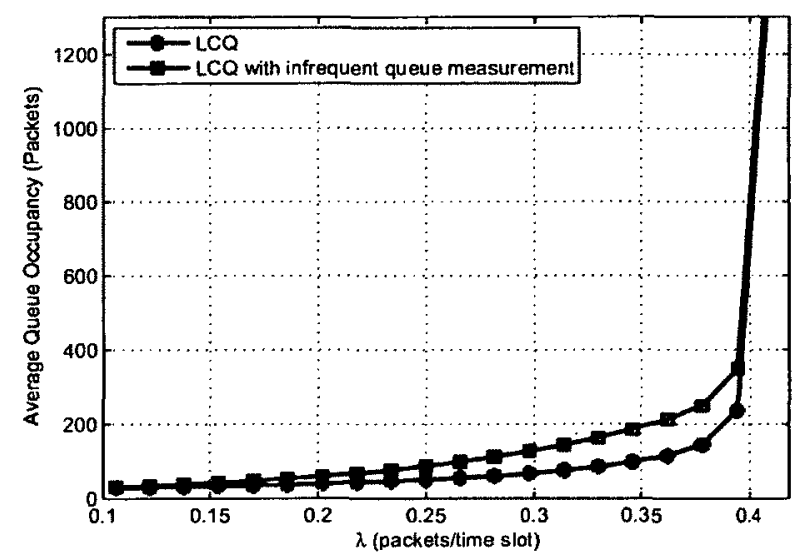

Figure 2.5: Delay comparison of LCQ with frequent and infrequent queue measurement

Minimizing the average queueing delay in wireless queueing systems has been studied in a few papers and just for some specific systems $[3,42,43,62,63,65]$. This is due to the fact that the objective function in this case is a strict objective function as we are looking for a policy that minimizes

$$
\limsup _{t \rightarrow \infty} \frac{1}{t} \sum_{\tau=0}^{t-1} \sum_{n=1}^{N} E\left[X_{n}(\tau)\right]
$$

Recall that in throughput optimization, we look for the policies that can keep the 
queue lengths bounded, i.e.,

$$
\limsup _{t \rightarrow \infty} \frac{1}{t} \sum_{\tau=0}^{t-1} \sum_{n=1}^{N} E\left[X_{n}(\tau)\right]<\infty
$$

for all the arrival rates in the stability region.

As explained earlier, the delay optimization problem has been just studied for some specific queueing systems. In [3], the authors studied the delay-optimal server allocation problem for a multi-queue single-server system with random connectivities and proved, using dynamic coupling and stochastic dominance arguments, that for a symmetric system with i.i.d. Bernoulli arrivals and connectivities, LCQ (Longest Connected Queue) policy provides the optimal performance in terms of average queue occupancy (or equivalently average queueing delay). Another relevant result is the work by Ganti et. al. [62] where the authors presented a model for a satellite node that has $K$ transmitters (servers). The satellite node is keeping $N$ queues and the data packets of each queue must be transmitted to a specific terrestrial node. It is assumed that the connectivity of the satellite node to each terrestrial node is changing randomly over time slots. At each time slot, no more than one transmitter is allocated to each queue. The authors proved that LCQ, a policy that allocates the $K$ transmitters to the $K$ longest connected queues at each time slot, is optimal (in stochastic ordering sense). References $[11,12,63,65]$ study the delay-optimal server allocation problem for multi-queue multi-server (MQMS) systems with random connectivities. In $[11,12,65]$, the authors argue that in general, achieving instantaneous throughput and load balancing is impossible in a general MQMS system. However, they showed that this goal is attainable in the special case with ON-OFF channel processes. They also introduced MTLB (Maximum-Throughput Load-Balancing) policy and showed that this policy is minimizing a class of cost functions including total average delay for the case of two symmetric queues. The work in [63] considers this problem for general number of symmetric queues and servers. The authors in [63] characterized a class of Most Balancing (MB) policies among all work-conserving policies which are minimizing, in stochastic ordering sense, a class of cost functions including total average delay. They used stochastic ordering and dynamic coupling arguments to show the optimality of MB policies for symmetric systems.

In Chapter 5, we will elaborate on the delay-optimal server assignment problem 
for a symmetric, multi-server, single-interface queueing system with random connectivities and show that Maximum Weighted Matching (MWM) policy not only is throughput-optimal but also is delay-optimal.

\subsection{Summary}

In this chapter, the background knowledge and related work for this thesis was presented by looking at the fundamental concepts of stability, stability region and throughput optimality. The back-pressure algorithm as a throughput-optimal resource allocation algorithm in queueing systems was further reviewed. We stated that the back-pressure algorithm does not need to know the stability region to act. However, in order to stabilize the system when the input arrival rates are outside the stability region, we need to solve network utility optimization problems where an explicit and precise description of the stability region in the form of convex equalities and inequalities is helpful. The general form of utility optimization problems in queueing systems was reviewed subsequently. Moreover, we stated some other applications of the explicit form of the stability region in performance evaluation of network control policies. The focus of the first part of this thesis would be on the stability analysis of multi-server queuing systems with time-varying channel conditions. Hence, a brief survey of the related work in this area of research was studied. Finally, we reviewed the notion of delay-optimal server allocation in wireless queuing systems and provided a brief literature survey in that regard. 


\section{Chapter 3}

\section{Stability Analysis of Randomly Connected Multi-server Queueing Systems}

In the previous chapter, the back-pressure algorithm was introduced as a throughputoptimal resource allocation policy for a general queueing network. We discussed that this algorithm only needs to have the instantaneous channel state and queue length information to work and does not need to have an explicit characterization of the stability region. However, it was shown that an explicit characterization of the stability region is useful for solving network utility optimization problems when the arrival rates are outside the system stability region. It is also advantageous for performance evaluation of network control policies.

In this chapter, we focus on the stability analysis of multi-server queueing systems with random connectivities between the queues and the servers. Specifically, we consider three multi-server queueing systems which are applicable to model the resource allocation problem in wireless communication networks. For each system, we will elaborate on the characterization of the stability region by use of a finite set of linear inequalities. The first system we considered is a multi-interface queueing system with a randomly connected server bank which can be used to model the transmitter allocation problem in a satellite node [62]. The second system is a multi-queue multi-server system with random connectivities which could be applied to model sub-carrier allocation problem in OFDMA (Orthogonal Frequency Division Multiple Access) networks [11-13,63,65]. Finally, the third system is a multi-server single-interface queueing system with random connectivities which is useful in modeling the channel allocation problem in wireless access networks [5]. This model is 
also applicable in modeling the relay selection problem in wireless relaying access networks [18]. For each system, we derive the necessary and sufficient conditions for the stability of the system for general packet arrival processes with finite second moment (Lemmas 3.2,3.3,3.5,3.6,3.9 and 3.10). It is shown (in Theorems 3.1, 3.2 and 3.3) that for stationary arrival processes, the necessary and sufficient conditions define the stability region of the system. The stability region in each case is characterized by a finite set of linear inequalities on the arrival rates. Hence, the stability region is the intersection of a finite set of half-spaces in the non-negative orthant with binary normal vectors and therefore the stability region is a convex polytope. We further obtain an upper bound, in an explicit form, for the average total queue occupancy for each system. Using simulations, we will evaluate the obtained occupancy bound and also compare (in terms of average queue occupancy and stability region) the performance of the throughput-optimal policy for each system with that of a randomized policy. The contents of this chapter require some mathematical background about the fundamental concepts of polytopes and also Lyapunov stability techniques. Therefore, the reader is encouraged to consult with Appendix A if needed.

\subsection{Multi-interface Queueing System with a Ran- domly Connected Server Bank}

In this section, we will elaborate on the characterization of the stability region for multi-interface queueing system with a randomly connected server bank. We start with describing the system in detail in the following subsection.

\subsubsection{System Description}

We consider a time-slotted queueing system with equal-length time slots. The system consists of a set of parallel queues denoted by $\mathcal{N}=\{1,2, \ldots, N\}$ competing to attract $K$ servers. The set of servers is denoted by $\mathcal{K}=\{1,2, \ldots, K\}$. The servers are assumed to form a server bank (see Figure 3.1). The queues are assumed to have infinite buffer space that keeps the packets arriving to the queues exogenously. The packets in the system are assumed to be of the same length. Each server is capable of serving one packet from one queue at each time slot. We assume that the exogenous arrival process to queue $n$ at time slot $t$ is denoted by $\left\{A_{n}(t)\right\}_{t=1}^{\infty}$. In fact, $A_{n}(t)$ represents 


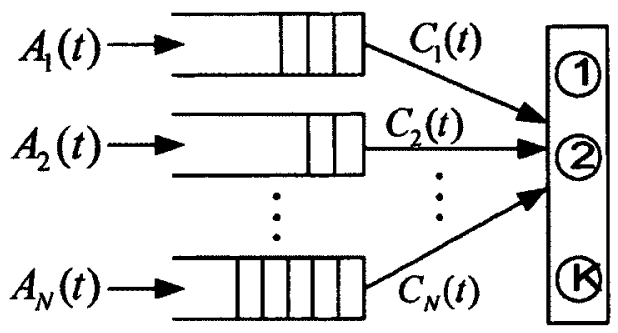

Figure 3.1: Multi-interface queueing system with a server bank and random connectivities

the number of packets arrived to queue $n$ during time slot $t$. The arrival processes are assumed to follow any general distribution with bounded second moment, i.e., $E\left[A_{n}^{2}(t)\right] \leq A_{\max }^{2}<\infty$ for all $t$.

The connectivity of the link from each queue $n$ to the server bank at time slot $t$ is denoted by random variable $C_{n}(t) \in\{0,1\}$. We assume that the connectivity of each queue $n$ to the server bank is a stationary binary random process denoted by $\left\{C_{n}(t)\right\}_{t=1}^{\infty}$ with stationary probabilities $p_{n}$ (i.e., $E\left[C_{n}(t)\right]=p_{n}$ for all $t$ ). The connectivity processes are assumed to be independent. Each queue is equipped with $R$ interfaces and thus it can make use of at most $R$ servers at each time slot. Note that for $R=1$, this model is equivalent to the model studied in [62] where it was used to study the problem of delay-optimal transmitter allocation in a satellite node. Our model generalizes the model used in [62] by considering $R \geq 1$ interfaces for each queue instead of only one interface. We complement the work in [62] by focusing on the stability region characterization which has not been addressed before.

During a time slot, we first serve the queues and then at the end of the time slot we add the new arrivals of the current time slot to the queues. The vector $X(t)=\left(X_{1}(t), X_{2}(t), \ldots, X_{N}(t)\right)$ denotes the queue length vector at the end of time slot $t$ after adding the arrivals of time slot $t$ to the queues.

A server allocation policy in this system determines the number of servers that must be allocated to each queue at each time slot. This is done based on the information regarding the connectivities and also the queue length vector at each time slot. Note that the number of allocated servers to each queue cannot be more than $R$ (i.e., the available interfaces for each queue). 
We introduce the indicator variables $I_{j, k}^{(n)}(t)$ as follows.

$$
I_{j, k}^{(n)}(t)=\left\{\begin{array}{lc}
1, & \text { Server } k \text { is allocated to interface } j \text { of queue } n \text { at time slot } t \\
0, & \text { otherwise. }
\end{array}\right.
$$

Therefore, a server allocation policy is equivalent to determination of the indicator variables $I_{j, k}^{(n)}(t), \forall n \in \mathcal{N}, \forall k \in \mathcal{K}, \forall j \in\{1,2, \ldots, R\}$ such that the following constraints are satisfied:

$$
\begin{aligned}
& \sum_{n=1}^{N} \sum_{j=1}^{R} I_{j, k}^{(n)}(t) \leq 1 \quad \forall k \in \mathcal{K} \\
& \sum_{k=1}^{K} I_{j, k}^{(n)}(t) \leq 1 \quad \forall n \in \mathcal{N}, \forall j=1, . ., R
\end{aligned}
$$

The sequence of the events in each time slot is summarized as follows:

- The server scheduler observes the connectivity variables $C_{n}(t), \forall n \in \mathcal{N}$ and the queue length vector at the beginning of time slot slot $t$, i.e., $X(t-1)$ and performs the server allocation process by determination of indicator variables $I_{j, k}^{(n)}(t)$. The queues are served accordingly.

- At the end of the time slot, the new arrivals are added to the queues and the queue length vector is updated. Let $H_{n}(t)$ be the departure process at time slot $t$ from queue $n$ due to the service provided by the server bank. Therefore, at the end of time slot $t$ the queue lengths are updated according to the following equation.

$$
X_{n}(t)=X_{n}(t-1)-H_{n}(t)+A_{n}(t) \quad \forall n \in \mathcal{N}
$$

\subsubsection{System Stability Analysis}

In this section, we will elaborate on the stability analysis of the system described above. We will introduce the necessary and sufficient conditions for the stability of this system. Before we proceed to the necessary conditions, we need to prove the following lemma for a strongly stable system. 
Lemma 3.1 If the system is strongly stable under some server allocation policy, then for each queue $n$ we have

$$
\overline{E\left[A_{n}(t)\right]}=\overline{E\left[H_{n}(t)\right]}
$$

The proof is presented in Appendix B.1. This lemma states that for a stable system the average expected arrivals to a queue is equal to the average expected departure from that queue.

For any subset of queues $Q \subseteq \mathcal{N}$, let $E_{i}^{Q}$ be the event that at least $i$ queues from subset $Q$ are connected to the server bank at a given time slot. Then, the necessary conditions for the stability of the system are given by the following lemma.

Lemma 3.2 Necessary Conditions for Stability: If the system is stable under some server allocation policy, then

$$
\overline{\sum_{n \in Q} E\left[A_{n}(t)\right]} \leq \sum_{i=1}^{|Q|} \min \left\{(K-(i-1) R)^{+}, R\right\} P\left(E_{i}^{Q}\right) \quad \forall Q \subseteq \mathcal{N}
$$

where $|Q|$ is the cardinality of $Q$.

The proof is presented in Appendix B.2.

Remark: For stationary arrival processes where we have $E\left[A_{n}(t)\right]=\lambda_{n}$ for all $n$, the necessary conditions for the stability of the system would be

$$
\sum_{n \in Q} \lambda_{n} \leq \sum_{i=1}^{|Q|} \min \left\{(K-(i-1) R)^{+}, R\right\} P\left(E_{i}^{Q}\right) \quad \forall Q \subseteq \mathcal{N}
$$

Note that the right-hand side of inequalities in (3.7) is the aggregated service provided to the queues of subset $Q$. In fact (3.7) intuitively indicates that the aggregated arrival rate for any subset of queues must be less than the aggregated service provided by the server bank to that subset of queues.

We can easily verify that the solution of the following optimization problem determines the indicator variables associated to the throughput-optimal policy (the back-pressure algorithm) for the multi-interface queueing system with a randomly connected server bank [4]. 


$$
\begin{aligned}
\text { Maximize } & \sum_{n=1}^{N} X_{n}(t-1) C_{n}(t) \sum_{j=1}^{R} \sum_{k=1}^{K} I_{j, k}^{(n)}(t) \\
\text { s.t. } & \sum_{n=1}^{N} \sum_{j=1}^{R} I_{j, k}^{(n)}(t) \leq 1 \quad \forall k=1,2, \ldots, K \\
& \sum_{k=1}^{K} I_{j, k}^{(n)}(t) \leq 1 \quad \forall n=1,2, \ldots, N \quad \forall j=1, \ldots, R
\end{aligned}
$$

Although this problem is an integer programming problem, it is not hard to show that the solution is equivalent to the following simple LCQ rule: Allocate $\min \{(K-$ $\left.\left.(n-1) R)^{+}, R\right)\right\}$ servers to the $n$th longest connected queue. Hence, we will allocate the servers from the server bank to the longest queues greedily. In particular, we will start from the longest connected queue and allocate as many servers to that queue as possible, i.e., $\min (K, R)$. For the second longest connected queue, we will follow the same rule. If the remainder of servers is greater than $R$, we will allocate $R$ servers to that queue. Otherwise, we will allocate all the remained servers to that queue. This procedure continues until we run out of servers. We call this policy LCQ as it is serving the longest connected queues greedily. This policy is in fact a generalization of LCQ policy which had been proposed in $[3,62]$ for $R=1$. In the following lemma, we will derive the sufficient conditions for the stability of multi-interface queueing system with a randomly connected server bank.

Lemma 3.3 Sufficient Conditions for Stability: The system is stable under LCQ policy if for all $t$

$$
\sum_{n \in Q} E\left[A_{n}(t)\right]<\sum_{i=1}^{|Q|} \min \left\{(K-(i-1) R)^{+}, R\right\} P\left(E_{i}^{Q}\right) \quad \forall Q \subseteq \mathcal{N}
$$


Furthermore, the following bound for the average expected "aggregate" occupancy holds.

$$
\begin{aligned}
& \limsup _{t \rightarrow \infty} \frac{1}{t} \sum_{\tau=0}^{t-1} \sum_{n=1}^{N} E\left[X_{n}(\tau)\right] \\
& \leq \frac{\frac{-N^{2}}{2}\left(A_{\max }^{2}+R^{2}\right)}{\max _{Q \subseteq\{1,2, \ldots, N\}, t}\left\{\sum_{n \in Q} E\left[A_{n}(t)\right]-\sum_{i=1}^{|Q|} \min \left\{(K-(i-1) R)^{+}, R\right\} P\left(E_{i}^{Q}\right)\right\}}
\end{aligned}
$$

The proof is given in Appendix B.3.

Remark: For stationary arrival processes where we have $E\left[A_{n}(t)\right]=\lambda_{n}$ for all $n$, the sufficient conditions for the stability of the system would be

$$
\sum_{n \in Q} \lambda_{n}<\sum_{i=1}^{|Q|} \min \left\{(K-(i-1) R)^{+}, R\right\} P\left(E_{i}^{Q}\right) \quad \forall Q \subseteq \mathcal{N}
$$

Based on the definition of stability region in Chapter 2 and the inequalities (3.7) and (3.11), the stability region of multi-interface queueing system with a randomly connected server bank is determined by the following theorem.

Theorem 3.1 The stability region of multi-interface queueing system with a randomly connected server bank is specified by the following series of linear inequalities.

$$
\sum_{n \in Q} \lambda_{n} \leq \sum_{i=1}^{|Q|} \min \left\{(K-(i-1) R)^{+}, R\right\} P\left(E_{i}^{Q}\right) \quad \forall Q \subseteq \mathcal{N}
$$

\subsection{Multi-queue Multi-server System with Random Connectivities}

In this section, we will elaborate on the characterization of the stability region of multi-queue, multi-server system with random connectivities. More specifically, we will derive the necessary and sufficient conditions for the stability of this system and finally conclude the stability region of the system. We further derive an upper bound, in an explicit form, for the average queue occupancy of the system. This queueing system has been studied in [5,11-13,63,65] for the modeling of OFDM sub-carrier allocation problem in wireless OFDM access networks. We will start with introducing 
the system in detail in the following subsection.

\subsubsection{System Description}

The system consists of a set of $N$ queues (which are modeling the wireless users) and a set of $K$ servers (which are modeling OFDM sub-carriers). The set of queues and servers are denoted by $\mathcal{N}=\{1,2, \ldots, N\}$ and $\mathcal{K}=\{1,2, \ldots, K\}$, respectively (similar to the previous system). The system is time-slotted with equal-length time slots and infinite buffer space for each queue, i.e., we do not have packet drops in the system. The exogenous arrival process to each queue $n$ is denoted by $\left\{A_{n}(t)\right\}_{t=1}^{\infty}$. Note that $A_{n}(t)$ represents the number of packet arrivals to queue $n$ during time slot $t$. The arrival process is assumed to follow a general distribution with bounded second moment, i.e., $E\left[A_{n}^{2}(t)\right] \leq A_{\max }^{2}<\infty$ for all $t$.

Each server can serve at most one packet at each time slot. We do not allow server sharing by the queues i.e., each server can serve at most one queue per time slot. At each time slot $t$, the link between each queue $n$ and server $k$ is either connected or disconnected. The connectivity process between queue $n \in \mathcal{N}$ and server $k \in \mathcal{N}$ is modeled by a stationary binary random process which is denoted by $\left\{C_{n, k}(t)\right\}_{t=1}^{\infty}$ where $C_{n, k}(t) \in\{0,1\}$. Assume that $E\left[C_{n, k}(t)\right]=p_{n, k}$ for all $t$.

The new arrivals are added to the queues at the end of each time slot. Let $X(t)=\left(X_{1}(t), X_{2}(t), \ldots, X_{N}(t)\right)$ be the queue length vector at the end of time slot $t$ after adding the new arrivals to the queues. Figure 3.2 depicts the model for the analysis of this section.

At the beginning of each time slot, a server allocation policy is employed to allocate the servers to the queues for service. Note that there is no limitation on the number of servers that can be allocated to each queue. A queue can thus utilize any number of available servers at each time slot. We introduce the indicator variables $I_{n, k}(t)$ as follows:

$$
I_{n, k}(t)=\left\{\begin{array}{lc}
1, & \text { Server } k \text { is allocated to queue } n \text { at time slot } t \\
0, & \text { otherwise }
\end{array}\right.
$$

Note that the indicator variables $I_{n, k}(t)$ are constrained to satisfy $\sum_{n=1}^{N} I_{n, k}(t) \leq 1$ for all $k=1,2, \ldots, K$. Therefore, any valid server allocation at time slot $t$ may be expressed by matrix $I(t)=\left(I_{n, k}(t), n \in \mathcal{N}, k \in \mathcal{K}\right)$ containing the valid indicator 


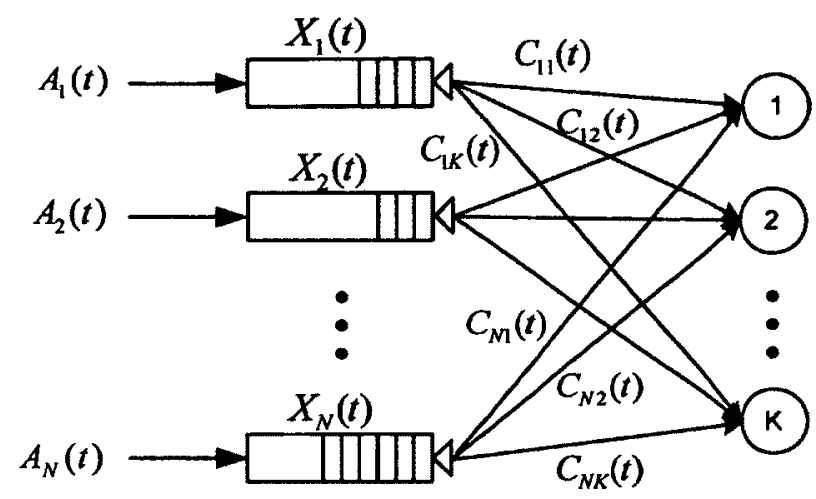

Figure 3.2: Multi-queue multi-server queueing system with random connectivities

variables $I_{n, k}(t)$.

In summary, the sequence of events during each time slot $t$ is as follows:

- The server scheduler observes the connectivity variables $C_{n, k}(t), \forall n \in \mathcal{N}, \forall k \in \mathcal{K}$ and the queue length vector at the beginning of time slot slot $t$, i.e., $X(t-1)$ and performs the server allocation process by determination of indicator variables $I_{n, k}(t)$. The queues are served accordingly.

- At the end of the time slot, the new arrivals of time slot $t$ are added to the queues and the queue length vector is updated. Let $H_{n, k}(t)$ be the departure process at time slot $t$ from queue $n$ due to the service provided by server $k$. Therefore, at the end of time slot $t$ the queue lengths are updated according to the following equation.

$$
X_{n}(t)=X_{n}(t-1)-\sum_{k=1}^{K} H_{n, k}(t)+A_{n}(t) \quad \forall n \in \mathcal{N}
$$

\subsubsection{System Stability Analysis}

In this section, we will elaborate on characterizing the stability region of multi-queue multi-server system with random connectivities. We will introduce the necessary and sufficient conditions for the stability of the system in Lemmas 3.5 and 3.6 and from them we will conclude the system stability region. The stability region will be 
characterized by a finite set of linear inequalities. Before we proceed to the necessary conditions, we will prove the following lemma for a strongly stable system.

Lemma 3.4 If the system is strongly stable under some server allocation policy, then for each queue $n$ we have

$$
\overline{E\left[A_{n}(t)\right]}=\overline{\sum_{k=1}^{K} E\left[H_{n, k}(t)\right]}
$$

The proof is skipped since the lemma follows by applying the same approach as we used for the proof of Lemma 3.1. This lemma states that for a stable system the average expected arrivals to a queue is equal to the average expected departure from that queue. Using Lemma 3.4, we can prove the following lemma indicating the necessary conditions for the stability of the system.

Lemma 3.5 Necessary Conditions for Stability: If the system is strongly stable under some server allocation policy, then

$$
\overline{\sum_{n \in Q} E\left[A_{n}(\tau)\right]} \leq \sum_{k=1}^{K}\left(1-\prod_{n \in Q}\left(1-p_{n, k}\right)\right) \quad \forall Q \subseteq \mathcal{N} .
$$

The proof is presented in Appendix B.4.

Remark: For stationary arrival processes where we have $E\left[A_{n}(t)\right]=\lambda_{n}$ for all $n$, the necessary conditions for the stability of the system would be

$$
\sum_{n \in Q} \lambda_{n} \leq \sum_{k=1}^{K}\left(1-\prod_{n \in Q}\left(1-p_{n, k}\right)\right) \quad \forall Q \subseteq \mathcal{N}
$$

In [13], it has been proven that the solution of the following optimization problem determines a throughput-optimal server allocation policy (back-pressure algorithm) for the multi-queue multi-server system with random connectivities.

$$
\begin{array}{ll}
\text { Maximize } & \sum_{n=1}^{N} X_{n}(t-1) \sum_{k=1}^{K} C_{n, k}(t) I_{n, k}(t) \\
\text { s.t. } & \sum_{n=1}^{N} I_{n, k}(t) \leq 1 \quad \forall k=1,2, \ldots, K
\end{array}
$$


This policy is called Maximum Weight (MW) policy since it maximizes the total weighted capacity of the system where the weights are equal to the queue lengths. The solution of this problem can be easily obtained by the following simple rule: Allocate each server to its longest connected queue. We now derive the sufficient conditions for the stability of the system and prove that Maximum Weight policy stabilizes the system as long as conditions (3.19) below are satisfied. An upper bound is also derived for the time averaged expected queue occupancy in the system.

Lemma 3.6 Sufficient Conditions for stability: The system is stable under Maximum Weight policy if for all $t$

$$
\sum_{n \in Q} E\left[A_{n}(t)\right]<\sum_{k=1}^{K}\left(1-\prod_{n \in Q}\left(1-p_{n, k}\right)\right) \quad \forall Q \subseteq \mathcal{N}
$$

Furthermore, the following bound for the average expected "aggregate" occupancy holds.

$$
\limsup _{t \rightarrow \infty} \frac{1}{t} \sum_{\tau=0}^{t-1} \sum_{n=1}^{N} E\left[X_{n}(\tau)\right] \leq \frac{-\frac{N}{2}\left(N A_{\max }^{2}+K(2 K-1)\right)}{\max _{Q \subseteq\{1,2, \ldots, N\}, t}\left\{\sum_{n \in Q} E\left[A_{n}(t)\right]-K+\sum_{k=1}^{K} \prod_{n \in Q}\left(1-p_{n, k}\right)\right\}}
$$

The proof is presented is Appendix B.5.

Remark: For stationary arrival processes where we have $E\left[A_{n}(t)\right]=\lambda_{n}$ for all $n$, the sufficient conditions for the stability of the system would be

$$
\sum_{n \in Q} \lambda_{n}<\sum_{k=1}^{K}\left(1-\prod_{n \in Q}\left(1-p_{n, k}\right)\right) \quad \forall Q \subseteq \mathcal{N}
$$

According to the definition of the stability region and the inequalities (3.17) and (3.21), we can conclude the following theorem.

Theorem 3.2 The stability region of multi-queue multi-server system with random connectivities is specified by the following series of inequalities.

$$
\sum_{n \in Q} \lambda_{n} \leq K-\sum_{k=1}^{K} \prod_{n \in Q}\left(1-p_{n, k}\right) \quad \forall Q \subseteq \mathcal{N}
$$


In this case, similar to the previous system we characterized the stability region by a finite set of linear inequalities on the arrival rates $\lambda_{n}$. Note that since the number of non-empty subsets of set $\mathcal{N}$ is equal to $2^{N}-1$, the stability region in both systems is characterized by $2^{N}-1$ half-spaces in $\mathbb{R}_{+}^{N}$ whose normal vectors belong to $\{0,1\}^{N}$.

\subsection{Multi-server Single-interface Queueing System with Random Connectivities}

In this section, we will consider the linear algebraic characterization of the stability region for a queueing system similar to the system described in Section 3.2.1 but with one interface per queue. Hence, the queues are constrained to exploit at most one server in each time slot. Note that in the system analysed in Section 3.2.1, the queues can get service from multiple servers in each time slot (upon the availability of servers and the decisions of the server allocation policy). Adding this constraint to the system makes the stability analysis of the system complicated such that we cannot use the same approach as we used in Sections 3.2.2 and 3.1.2. For the analysis of this section, we will incorporate both Lyapunov drift stability analysis and linear algebraic arguments to prove the necessary and sufficient conditions for the stability of the system. We will start with detailed description of the system in the following subsection.

\subsubsection{System Description}

Consider a time slotted multi-server queueing system similar to the system of Section 3.2.1 (See Figure 3.2) with single-interface queues. The set of queues and the set of servers are denoted by $\mathcal{N}=\{1,2, \ldots, N\}$ and $\mathcal{K}=\{1,2, \ldots, K\}$, respectively. The length of time slots and the packets are assumed to be fixed. Each server is capable of serving at most one packet from a single queue at each time slot. We assume that the queues are single-interface queues, i.e., each queue can get service from at most one server during each time slot. Such multi-server queueing systems can be applied in modeling the resource allocation problem (e.g., relay stations and communication channels) in wireless networks $[5,18]$.

The connectivity of each queue $n \in \mathcal{N}$ to each server $k \in \mathcal{K}$ at time slot $t$ is random and varying across time slots. We denote the connectivity of queue $n$ to 
server $k$ at time slot $t$ by $C_{n, k}(t) \in\{0,1\}$. At each time slot $t$, the connectivity state may be expressed by an $N \times K$ matrix $C(t)=\left(C_{n, k}(t), n \in \mathcal{N}, k \in \mathcal{K}, C_{n, k}(t) \in\{0,1\}\right)$. Therefore, the connectivity process is defined as $\{C(t)\}_{t=1}^{\infty}$ with the state space $\mathcal{C}$ (i.e., $\mathcal{C}$ is the set of all $N \times K$ binary matrices). Note that $\mathcal{C}$ is a finite set with $|\mathcal{C}|=2^{N K}$. The connectivity process is assumed to have stationary distribution with stationary probabilities $P(c)=\operatorname{Pr}(C(t)=c)$. In fact, $P(c)$ represents the fraction of time slots where the connectivity of the system is in state $c$. Note that the connectivities of different links in the system may be arbitrary correlated during a time slot (in contrast to the system of Section 3.2). Thus, we consider a weaker assumption for the connectivity processes with respect to the system of Section 3.2.

The exogenous arrival process to each queue $n$ is denoted by $\left\{A_{n}(t)\right\}_{t=1}^{\infty} . A_{n}(t)$ represents the number of packet arrivals to queue $n$ during time slot $t$. The arrival process is assumed to follow a general distribution with bounded second moment, i.e., $E\left[A_{n}^{2}(t)\right] \leq A_{\max }^{2}<\infty$ for all $t$. Each queue has an infinite buffer space i.e., we do not have packet drop in the system. We assume that the new arrivals are added to each queue at the end of each time slot. Let $X(t)=\left(X_{1}(t), X_{2}(t), \ldots, X_{N}(t)\right)$ be the queue length vector at the end of time slot $t$ after adding new arrivals to the queues.

A server assignment policy at each time slot determines an assignment of servers of set $\mathcal{K}$ to the queues of set $\mathcal{N}$. Thus, at each time slot the scheduler has to decide about a bipartite matching (matching in bipartite graphs ${ }^{1}$ ) between sets $\mathcal{N}$ and $\mathcal{K}$. This should be accomplished based on the available information regarding the connectivities $C_{n, k}(t)$ and also the queue length vector at the beginning of time slot $t$, i.e., $X(t-1)=\left(X_{1}(t-1), X_{2}(t-1), \ldots, X_{N}(t-1)\right)$. For a given policy $\pi$, suppose that indicator variable $I_{n, k}^{(\pi)}(t)$ is defined to be " 1 " if server $k$ is assigned to queue $n$ at time slot $t$ and " 0 " otherwise. We define the $N \times K$ matrix $I^{(\pi)}(t)=\left(I_{n, k}^{(\pi)}(t), \forall n \in \mathcal{N}, k \in \mathcal{K}\right)$ as the employed matching by policy $\pi$ at time slot $t$. Therefore, a server scheduling policy $\pi$ is defined as $\pi=\left\{I^{(\pi)}(t)\right\}_{t=1}^{\infty}$. We denote the matching space, i.e., the set of all the possible matchings in an $N \times K$ bipartite graph by $\mathcal{I}$. In other words,

$\mathcal{I}=\left\{I_{N \times K}=\left(I_{n, k} \forall n \in \mathcal{N}, k \in \mathcal{K}\right) \mid \sum_{k=1}^{K} I_{n, k} \leq 1 \forall n \in \mathcal{N}, \sum_{n=1}^{N} I_{n, k} \leq 1 \forall k \in \mathcal{K}\right\}$

According to the above discussion, we can see that the queue length random

\footnotetext{
${ }^{1}$ A matching in a bipartite graph is a sub-graph of the original graph in which no two edges share a common vertex.
} 
process $X_{n}(t)$, evolves with time according to the following rule.

$$
X_{n}(t)=\left(X_{n}(t-1)-\sum_{k=1}^{K} C_{n, k}(t) I_{n, k}^{(\pi)}(t)\right)^{+}+A_{n}(t) \quad \forall n \in \mathcal{N}
$$

Note that a server can be assigned to an empty queue however it cannot serve it since there is no packet to be served. That is why we have used operator $(\cdot)^{+}$in $(3.23)$.

As we discussed earlier, the queueing model introduced in this section is useful in modeling the resource assignment problem in various systems with shared resources. In wireless communication systems, communication resources such as communication sub-channels, relay stations, etc. are shared among users and therefore resource assignment problem in these networks can be studied using our model (e.g., $[5,18]$ ). For example, consider a relying access network with $N$ users and $K$ relays. By modeling the cooperative wireless channel between each user, each relay and the base station as a binary erasure channel, we can apply the introduced queueing model for performance evaluation.

\subsubsection{System Stability Analysis}

In this section, we will elaborate on characterizing the stability region of the system described above. We will introduce the necessary and sufficient conditions for the stability of the system (See Lemmas 3.9 and 3.10) and from them we will conclude the system stability region. The stability region will be characterized by a set of linear inequalities. As we explained earlier, our approach to obtain the necessary conditions for the stability of the system is different from what was used for the previous systems. We propose a linear algebraic methodology for proving the necessary conditions for the stability of the system. The proposed methodology is novel and can be applied for proving more complicated systems as well. We will use the same methodology in our analysis in Chapter 4.

Our steps in proving the necessary conditions for the stability of the system are summarized as follows:

- We will characterize the region of achievable service rates as a convex polytope using the approach introduced in Section 2.2.2. We will see that the region of achievable service rates is a convex polytope in the non-negative orthant. 
- We will determine the set of all the face-defining hyperplanes (Refer to Appendix A.2 for the definition of face and facet) associated to the rate region (stability region) polytope. This set is an infinite set.

- Among all the face-defining hyperplanes, we will show that just the hyperplanes with binary normal vectors are determining the facet-defining hyperplanes associated to the stability region polytope. Since a polytope can be completely characterized by its facet-defining hyperplanes, other face-defining hyperplanes are redundant (Refer to Appendix A.2 for the definition of redundant inequality) and therefore the stability region is characterized by $2^{N}-1$ linear inequalities.

\section{Region of Achievable Service Rates}

Consider the class of deterministic server assignment policies $\mathcal{G}$ in which at each time slot $t$, a policy $g \in \mathcal{G}$ assigns the servers to the queues according to a predetermined matching depending on the connectivity state of the system (i.e., matrix $C(t)$ ). More specifically, for each policy $g \in \mathcal{G}$, there exists a one-to-one mapping from the connectivity matrix space $\mathcal{C}$ to the matching space $\mathcal{I}$, namely $I^{(g)}: \mathcal{C} \longmapsto \mathcal{I}$. At each time slot $t$, the scheduler that is employing server assignment policy $g$ observes the connectivity matrix of the system, i.e., $c(t) \in \mathcal{C}$ and assigns the servers to the queues according to matching $I^{(g)}(c(t))$. Note that since $|\mathcal{I}|<\infty$ and $|\mathcal{C}|<\infty$, set $\mathcal{G}$ of all the deterministic server assignment policies is finite.

Each deterministic policy $g$ provides an average service rate for each queue $n$. Let $R_{n}^{(g)}$ denote the average service rate provided to queue $n$ and $R^{(g)}=$ $\left(R_{1}^{(g)}, R_{2}^{(g)}, \ldots, R_{N}^{(g)}\right)$ the vector of average service rates provided by policy $g$. By conditioning on $C(t)$, we can readily show that for each deterministic server assignment policy $g, R^{(g)}$ is obtained by the following equation.

$$
R^{(g)}=\left(\sum_{c \in \mathcal{C}} P(c)\left(c \circledast I^{(g)}(c)\right) \underline{1}_{K}^{\mathrm{T}}\right)^{\mathrm{T}}
$$

Note that each rate vector $R^{(g)}$ determines a single point in $\mathbb{R}_{+}^{N}$. Now, consider the convex hull of all the points $R^{(g)}, g \in \mathcal{G}$ in $\mathbb{R}_{+}^{N}$, i.e.,

$$
\mathcal{R}=\underset{g \in \mathcal{G}}{\operatorname{conv} . \operatorname{hull}\left(R^{(g)}\right)}
$$


Set $\mathcal{R}$ contains all the achievable service rate vectors for the multi-server singleinterface queueing system. Each point $R^{\star}$ in $\mathcal{R}$ can be represented by a convex combination of a finite set of points, $\left\{R^{(g)}, g \in \mathcal{G}\right\}$, i.e.,

$$
R^{\star}=\sum_{i=1}^{|\mathcal{G}|} p_{i} R^{g_{i}}, g_{i} \in \mathcal{G}, \sum_{i=1}^{|\mathcal{G}|} p_{i}=1, p_{i} \geq 0
$$

Hence, in order to achieve an arbitrary service rate vector $R^{\star}$, it is enough to select policy $g_{i}$ in $p_{i}$ fraction of time slots (a randomized policy). According to the definition of convex polytope $[85,86]$ and the fact that set $\mathcal{G}$ is finite, set $\mathcal{R}$ represents a convex polytope in $\mathbb{R}_{+}^{N}$.

\section{Linear Algebraic Representation of Stability Region}

In the following, we will characterize the stability region of the multi-server singleinterface queueing system with random connectivities by a finite set of linear inequalities. We introduce the departure matrix $H_{N \times K}(t)=\left(H_{n, k}(t), n \in \mathcal{N}, k \in \mathcal{K}\right)$ in which $H_{n, k}(t)$ represents the number of packets served by server $k$ from queue $n$ at time slot t. Obviously, $H_{n, k}(t) \in\{0,1\}$ and $H_{n, k}(t) \leq C_{n, k}(t) \leq 1$. Hence, the departure process from queue $n$ at time slot $t$ can be written as $\sum_{k=1}^{K} H_{n, k}(t)$. The following equation describes the evolution of the queue length process in time.

$$
X_{n}(t)=X_{n}(t-1)-\sum_{k=1}^{K} H_{n, k}(t)+A_{n}(t) \quad \forall n \in \mathcal{N}
$$

For a strongly stable queueing system the following is true.

Lemma 3.7 If the multi-server, single-interface queueing system described in Section 3.3 .1 is strongly stable under a server assignment policy, then for any vector $\alpha=$ $\left(\alpha_{1}, \alpha_{2}, \ldots, \alpha_{N}\right) \in \mathbb{R}^{N}$ we have

$$
\overline{\alpha E[A(t)]^{\mathrm{T}}}=\overline{\alpha E\left[H(t) \underline{1}_{K}^{\mathrm{T}}\right.}
$$

The proof is given in Appendix B.6.

In other words, for a strongly stable system the weighted arrival rate to the queues is equal to the time averaged weighted expected departure from the queues. We 
further can prove the following lemma. Note that this lemma is a generalization of Lemmas 3.1 and 3.4 .

Lemma 3.8 If the multi-server single-interface queueing system described in Section 3.3.1 is strongly stable under a server assignment policy, then for all $\alpha \in \mathbb{R}_{+}^{N}$

$$
\overline{\alpha E[A(t)]^{\mathrm{T}}} \leq \sum_{c \in \mathcal{C}} P(c) \max _{I \in \mathcal{I}}\left(\alpha(c \circledast I) \underline{1}_{K}^{\mathrm{T}}\right)
$$

The proof is given in Appendix B.7.

As we can see, the set of inequalities in (3.29) is an infinite set. Note that each inequality in (3.29) determines a valid inequality for polytope $\mathcal{R}$ (Refer to Appendix A.2 for the definition of valid inequality). It can be shown that the hyperplanes associated to the valid inequalities of (3.29) are all face-defining hyperplanes of polytope $\mathcal{R}$. Therefore, the hyperplane associated to each of the valid inequalities of (3.29) touches polytope $\mathcal{R}$ at least at one point. Towards this, let $\mathcal{I}^{\alpha}$ denote the set of matchings $\left\{I^{\alpha}(c), c \in \mathcal{C}\right\}$ that maximizes $\alpha(c \circledast I) \underline{1}_{K}^{\mathrm{T}}$, i.e.,

$$
I^{\alpha}(c)=\arg \max _{I \in \mathcal{I}} \alpha(c \circledast I) \underline{1}_{K}^{\mathrm{T}}
$$

Note that $\mathcal{I}^{\alpha}$ is not unique and there may be more than one set of matchings $\mathcal{I}^{\alpha}$ whose elements maximize $\alpha(c \circledast I) \underline{1}_{K}^{\mathrm{T}}$. According to (3.24) and (3.25), $\quad\left(\sum_{c \in \mathcal{C}} P(c)\left(c \circledast I^{\alpha}(c)\right) \underline{1}_{K}^{\mathrm{T}}\right)^{\mathrm{T}} \in \mathcal{R}$. Furthermore, since $\sum_{c \in \mathcal{C}} P(c) \alpha(c \circledast$ $\left.I^{\alpha}(c)\right) \underline{1}_{K}^{\mathrm{T}}=\alpha \sum_{c \in \mathcal{C}} P(c)\left(c \circledast I^{\alpha}(c)\right) \underline{1}_{K}^{\mathrm{T}}$, according to (3.29) this point (i.e., $\left.\left(\sum_{c \in \mathcal{C}} P(c)\left(c \circledast I^{\alpha}(c)\right) \underline{1}_{K}^{\mathrm{T}}\right)^{\mathrm{T}}\right)$ is located on the hyperplane associated to (3.29). Hence, the set of inequalities of (3.29) determines all the non-empty faces of polytope $\mathcal{R}$. To clarify what was proven in Lemma 3.8 , consider the two-queue single-server example again.

Example: Consider the two-queue single-server system $(N=2, K=1)$ described in Chapter 2. Assume that queues 1 and 2 are connected to the server with probabilities $p_{1}$ and $p_{2}$, respectively (see Figure 3.3 ). 


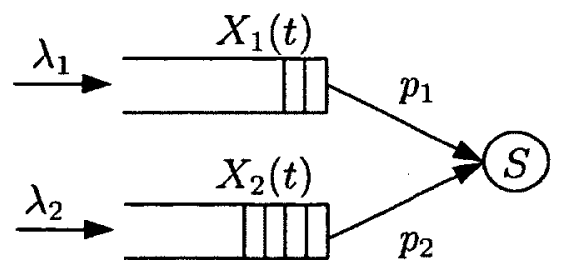

Figure 3.3: Two-queue single-server example

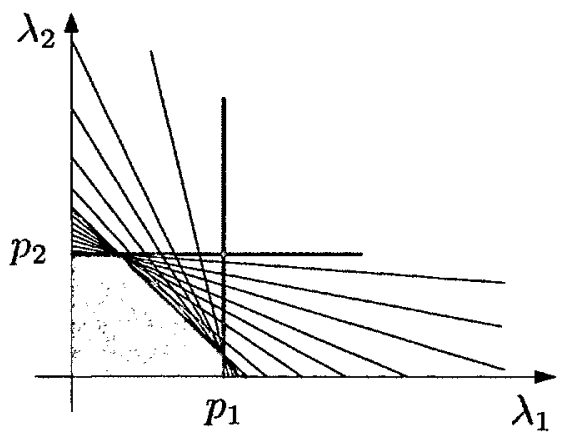

Figure 3.4: Stability region for the queueing system of Figure 3.3

For such a system it was proven that the stability region is described by the following set of inequalities.

$$
\begin{gathered}
\lambda_{1} \leq p_{1} \\
\lambda_{2} \leq p_{2} \\
\lambda_{1}+\lambda_{2} \leq p_{1}+p_{2}-p_{1} p_{2}
\end{gathered}
$$

The stability region is depicted in Figure 3.4 by the blue area. As we can see, for this example the stability region is a pentagon which is a 2-D convex polytope. In Lemma 3.8, the stability region was characterized by an infinite number of its facedefining hyperplanes. In Figure 3.4, we have also illustrated some of the face-defining hyperplanes of the stability pentagon derived according to (3.29) for some sample values of $\alpha=\left(\alpha_{1}, \alpha_{2}\right)$. According to redundancy theorem in polytopes (Refer to Appendix A.2 and also $[85,86])$, not all the face-defining hyperplanes of a polytope are necessary to describe a polytope, i.e., the inequalities corresponding to the facets of a polytope are sufficient to characterize a polytope. In the above example, the stability region can be completely determined by just three facet-defining hyperplanes illustrated by red lines in Figure 3.4. In the following lemma, we will show that not 
all $\alpha \in \mathbb{R}_{+}^{N}$ are required to describe polytope $\mathcal{R}$. In fact, Lemma 3.9 states that just $\alpha \in\{0,1\}^{N}-\left\{\underline{0}_{N}\right\}$ are sufficient to describe facet-defining hyperplanes of polytope $\mathcal{R}$.

Lemma 3.9 If the multi-server single-interface queueing system described in Section 3.3.1 is strongly stable under a server assignment policy, then

$$
\overline{\alpha E[A(t)]^{\mathrm{T}}} \leq \sum_{c \in \mathcal{C}} P(c) \max _{I \in \mathcal{I}}\left(\alpha(c \circledast I) \underline{1}_{K}^{\mathrm{T}}\right), \quad \alpha \in\{0,1\}^{N}-\left\{\underline{0}_{N}\right\}
$$

The proof is given in Appendix B.8.

Remark: For stationary arrival processes where we have $E\left[A_{n}(t)\right]=\lambda_{n}$ for all $n$, the necessary conditions for the stability of the system would be

$$
\alpha \lambda^{\mathrm{T}} \leq \sum_{c \in \mathcal{C}} P(c) \max _{I \in \mathcal{I}}\left(\alpha(c \circledast I) \underline{1}_{K}^{\mathrm{T}}\right), \quad \alpha \in\{0,1\}^{N}-\left\{\underline{0}_{N}\right\}
$$

Note that Lemma 3.9 describes the necessary conditions for the stability of the system. For completeness of our study we also have to show that all the rate vectors inside polytope $\mathcal{R}$ are rate stable. In other words, there exists a server assignment policy that can stabilize the system for all the rate vectors inside polytope $\mathcal{R}$. As we explained in Section 2.2.3, the back-pressure algorithm has been shown to be a throughput-optimal resource allocation in queueing networks. For the queueing system introduced in section 3.3.1, the back-pressure algorithm is equivalent to solving the following optimization problem at each time slot $t[5]$.

$$
\begin{aligned}
\text { Maximize: } \quad & \sum_{n=1}^{N} X_{n}(t-1) \sum_{k=1}^{K} I_{n, k}(t) C_{n, k}(t) \\
\text { Subject to: } \quad & \sum_{k=1}^{K} I_{n, k}(t) \leq 1 \quad(n=1,2 . ., N) \\
& \sum_{n=1}^{N} I_{n, k}(t) \leq 1 \quad(k=1,2 . ., K),
\end{aligned}
$$

Note that finding the solution of problem (3.34) is equivalent to finding a maximum weighted matching in the $N \times K$ bipartite graph $G_{t}=(\mathcal{N}, \mathcal{K}, \mathcal{E})$ shown in Figure 3.5. Therefore, this policy is called Maximum Weighted Matching (MWM) policy. 


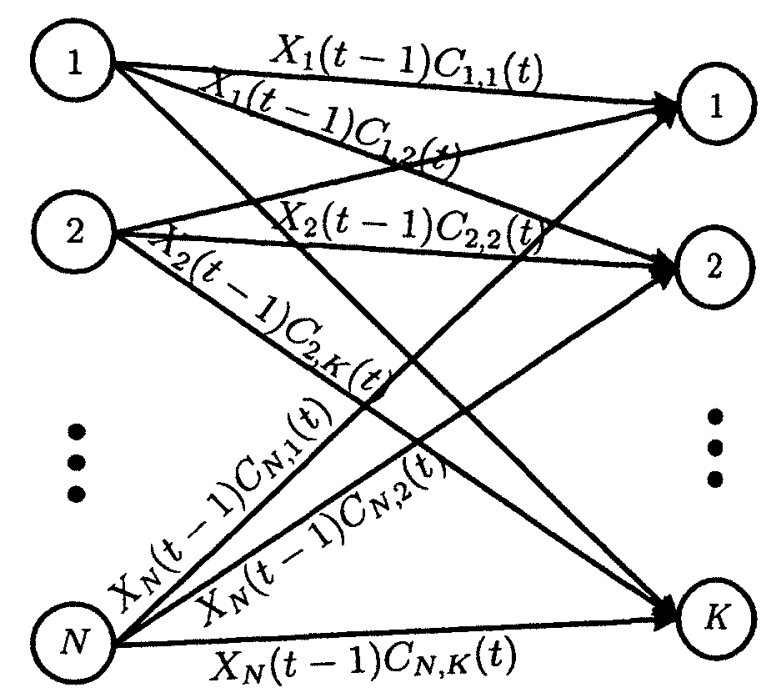

Figure 3.5: Bipartite graph corresponding to problem (3.34)

In $G_{t}, \mathcal{N}$ and $\mathcal{K}$ are the two sets of vertices in each part of the graph and $\mathcal{E}=$ $\left\{e_{n, k}, \forall n \in \mathcal{N}, \forall k \in \mathcal{K}\right\}$ is the set of edges between these two parts. Note that in $G_{t}$, the associated weight to each edge $e_{n, k}$ is $X_{n}(t-1) C_{n, k}(t)$. A matching in graph $G_{t}$ is a sub-graph of $G_{t}$ in which no two edges share a common vertex. Any matching $I^{(\pi)}(t)$ at any time slot $t$ is corresponding to a sub-graph of $G_{t}$ namely $G_{t}^{(\pi)}=\left(\mathcal{N}, \mathcal{K}, \mathcal{E}^{(\pi)}\right)$ in which $e_{n, k} \in \mathcal{E}^{(\pi)}$ if and only if $I_{n, k}^{(\pi)}(t)=1$.

There are several algorithms to find the maximum weighted matching in bipartite graphs. The most well known algorithm is Hungarian algorithm whose complexity is of $O\left((\min \{N, K\})(\max \{N, K\})^{2}\right)[87]$.

In the following lemma, we will prove that for a system satisfying conditions (3.35) below (which is describing $\mathcal{R}$ - bound(R)), MWM policy stabilizes the system. We further obtain an upper bound, in an explicit form, for the average expected aggregate backlog in the system.

Lemma 3.10 The multi-server single-interface queueing system described in Section 3.3.1 is stable under $M W M$ if

$$
\alpha E[A(t)]^{\mathrm{T}}<\sum_{c \in \mathcal{C}} P(c) \max _{I \in \mathcal{I}}\left(\alpha(c \circledast I) \underline{1}_{K}^{\mathrm{T}}\right), \quad \alpha \in\{0,1\}^{N}-\left\{\underline{0}_{N}\right\} .
$$


Furthermore, the following bound for the average expected "aggregate" occupancy holds.

$$
\limsup _{t \rightarrow \infty} \frac{1}{t} \sum_{\tau=0}^{t-1} \sum_{n=1}^{N} E\left[X_{n}(\tau)\right] \leq \frac{N A_{\max }^{2}+(\min \{N, K\})^{2}}{2 \delta}
$$

where $\delta=\min _{t, \alpha \in\{0,1\}^{N}-\left\{\underline{0}_{N}\right\}}\left\{\frac{1}{\sum_{i=1}^{N} \alpha_{i}}\left(\sum_{c \in \mathcal{C}} P(c) \max _{I \in \mathcal{I}}\left(\alpha(c \circledast I) \underline{\mathbb{1}}_{K}^{\mathrm{T}}\right)-\alpha E[A(t)]^{\mathrm{T}}\right)\right\}$.

The proof is provided in Appendix B.9.

Remark: For stationary arrival processes where we have $E\left[A_{n}(t)\right]=\lambda_{n}$ for all $n$, the sufficient conditions for the stability of the system would be

$$
\alpha \lambda^{\mathrm{T}}<\sum_{c \in \mathcal{C}} P(c) \max _{I \in \mathcal{I}}\left(\alpha(c \circledast I) \underline{1}_{K}^{\mathrm{T}}\right), \quad \alpha \in\{0,1\}^{N}-\left\{\underline{0}_{N}\right\} .
$$

According to the definition of the stability region, (3.33) and (3.37) we can conclude the following theorem.

Theorem 3.3 The stability region of multi-server single-interface queueing system with random connectivities is specified by the following series of inequalities.

$$
\alpha \lambda^{\mathrm{T}} \leq \sum_{c \in \mathcal{C}} P(c) \max _{I \in \mathcal{I}}\left(\alpha(c \circledast I) \underline{1}_{K}^{\mathrm{T}}\right), \quad \alpha \in\{0,1\}^{N}-\left\{\underline{0}_{N}\right\}
$$

\subsection{Numerical Results}

In Theorems 3.1, 3.2 and 3.3, the stability region of each multi-server system was characterized by a set of linear inequalities on the arrival rates. The number of linear inequalities for each case is equal $2^{N}-1$ which equals the number of non-empty subsets of the set of queues. Each of the inequalities in (3.12), (3.22) and (3.38) is a half-space in $\mathbb{R}_{+}^{N}$ and therefore the set of all the inequalities given in (3.12), (3.22) and (3.38) determines a convex polytope in $\mathbb{R}_{+}^{N}[85,86]$. Thus, the stability region of each system is a convex polytope in $\mathbb{R}_{+}^{N}$. The stability region polytope can be displayed for $N \leq 3$. In Figure 3.6, we show representative examples of the stability region of each system for $N=3$.

To evaluate the bounds on the total queue occupancy we derived earlier, we performed simulations where we selected the connectivity probabilities randomly in $[0,1]$. 


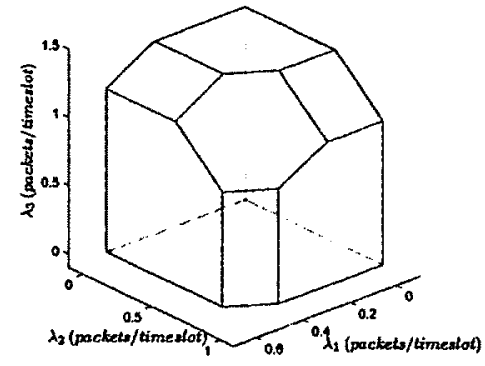

(a) Multi-interface queueing system with a randomly connected server bank $(N=3, R=2$ and $K=3)$

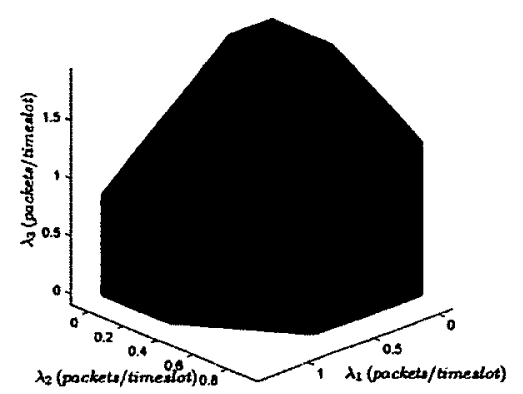

(b) Multi-queue multi-server system $(N=3, K=3)$

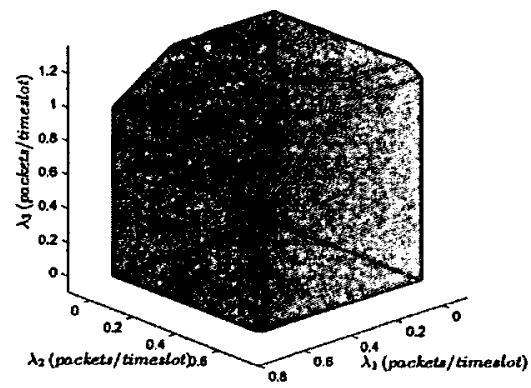

(c) Multi-server Single-interface system $(N=3$ and $K=3)$

Figure 3.6: Stability regions of studied multi-server queueing systems for sample values of $N, K, R$

For the multi-server single-interface system we selected a random stationary distribution for the connectivity matrix. For the arrival process $\left\{A_{n}(t)\right\}_{t=1}^{\infty}$ to each queue $n$ we assume that $A_{n}(t)$ 's are i.i.d. Poisson random variables with mean $\lambda_{n}=\lambda$ for all $n$, i.e., $E\left[A_{n}(t)\right]=\lambda$. For each system, the average total queue occupancy versus $\lambda$ was measured and compared with the obtained bounds in (3.10), (3.20) and (3.36) for sample values of $N, K$ and $R$. Figures 3.7-a, 3.7-b and 3.7-c show these results. We can observe that the obtained bounds for the average queue occupancy are not tight. Therefore, a future step in this area of research could be to further sharpen these bounds. Since the arrival rates to the queues are the same, the stability region is specified by just an inequality $\lambda \leq \lambda^{\star}$ (See Figures 3.7-a, 3.7-b and 3.7-c). We observe that as the input rates get closer to the stability point, the average total queue occupancy starts growing.

We also compared the performance of the optimal policies (i.e., LCQ, MW and MWM policies) with that of a randomized policy. For the multi-interface system with a randomly connected server bank, the randomized policy is defined as follows: At each time slot, each server is allocated randomly to one of the connected queues to the server bank that has available interfaces to get service. For the multi-queue multi-server system, the randomized policy is defined as follows: At each time slot, each server is allocated to one of the connected queues to that server randomly. For the multi-server single-interface system, the randomized policy is a random matching 


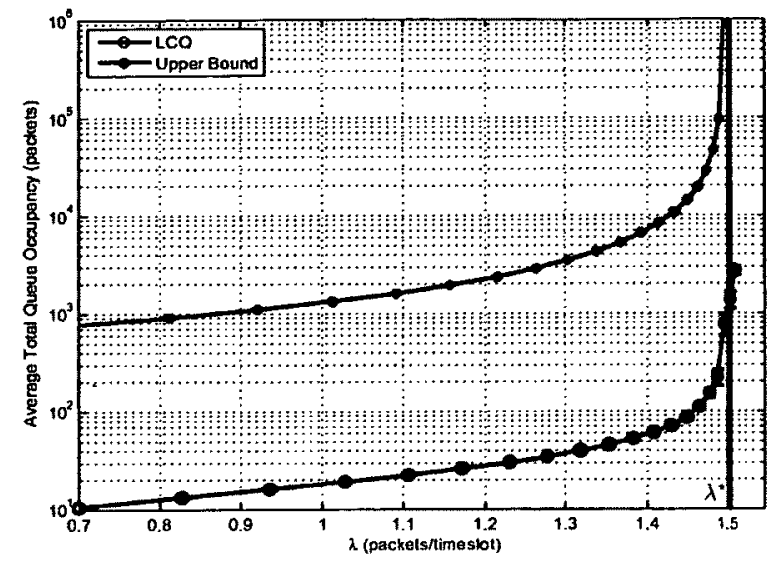

(a) Comparison of the performance of the LCQ policy with the obtained upper bound

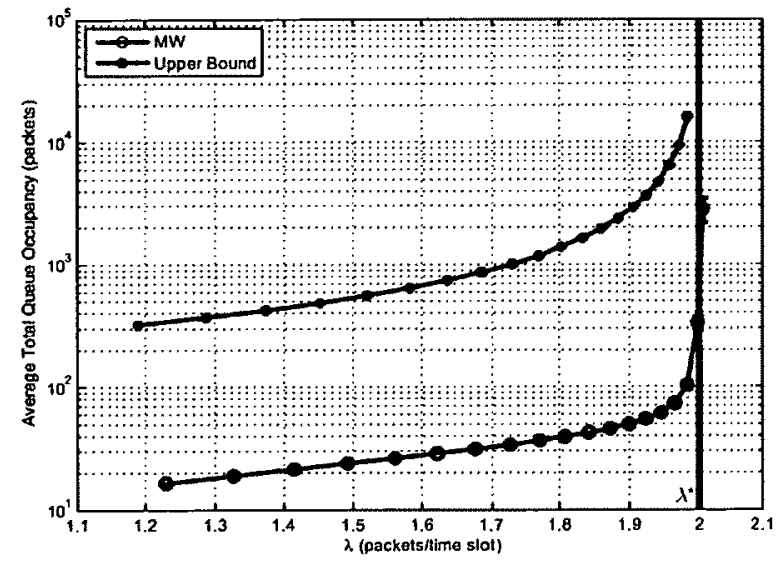

(b) Comparison of the performance of the MW policy with the obtained upper bound

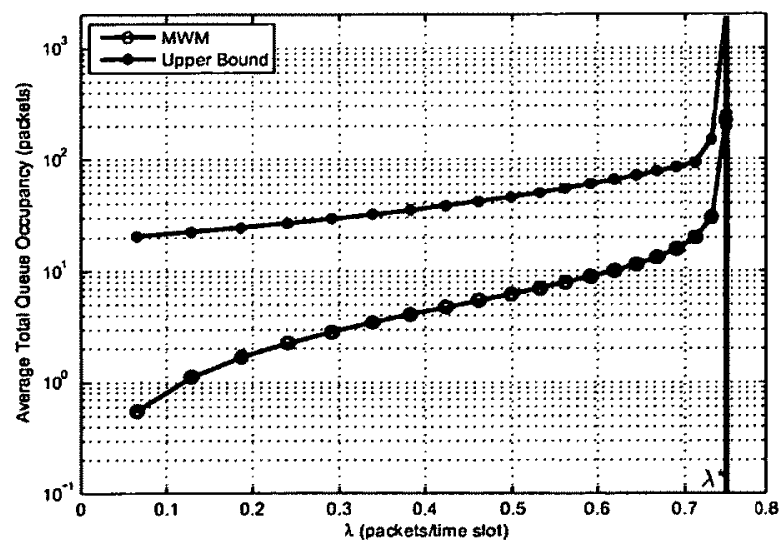

(c) Comparison of the performance of the MWM policy with the obtained upper bound

Figure 3.7: Evaluation of the obtained upper bounds 


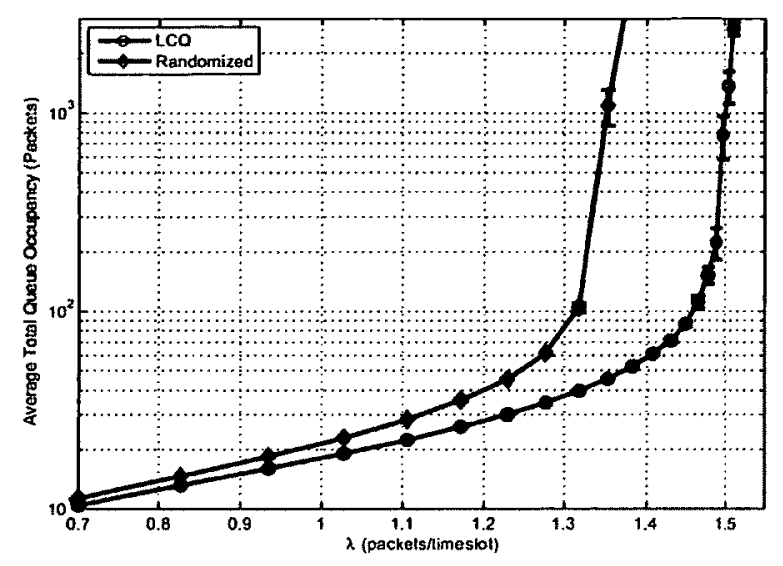

(a) Comparison of the performance of LCQ with a randomized policy

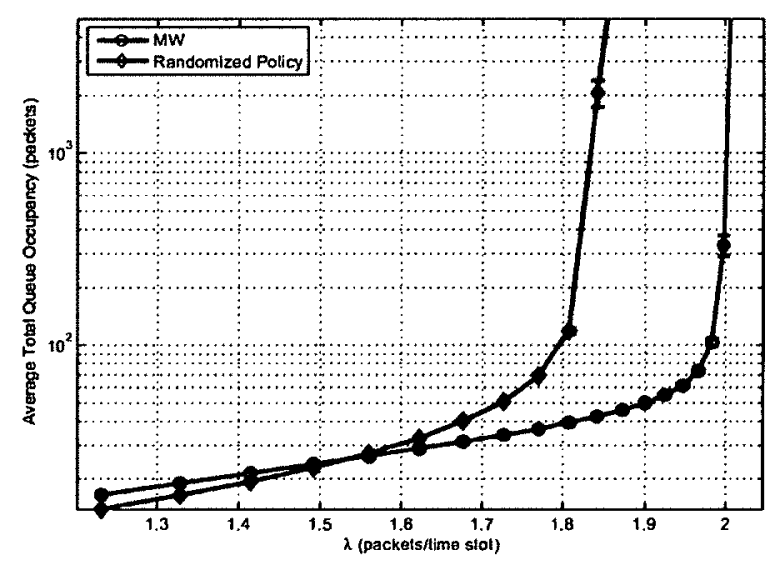

(b) Comparison of the performance of MW with a randomized policy

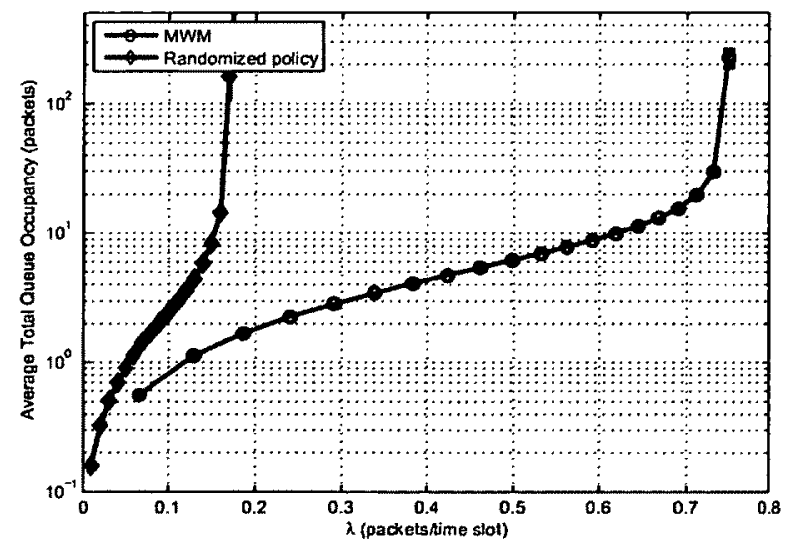

(c) Comparison of the performance of MWM with a randomized policy

Figure 3.8: throughput-optimal policy vs. randomized policy 
between the queues and the servers. Figures 3.8-a, 3.8-b and 3.8-c compare the performance of the optimal policies with the randomized policies. As expected the optimal policy in all the systems outperforms the randomized policy. Furthermore, we observe that the stability region of the randomized policy is less than the optimal one, i.e., the queues start growing faster under the randomized server allocation policy.

\subsection{Summary}

In this chapter, we characterized the stability regions of three randomly connected multi-server systems by a set of linear inequalities. The studied systems are applied to model the resource allocation problem in the emerging wireless communication systems. For each system, we derived the necessary and sufficient conditions for the stability of the system for general arrival processes with bounded second moment. Then, from those conditions we concluded the stability region of each system for stationary arrival processes. We showed that the stability region in each case is characterized by a finite set of linear inequalities. We also introduced an upper bound, in an explicit form, for the average queue occupancy of each system. 


\section{Chapter 4}

\section{Explicit Characterization of Stability Region for Stationary Multi-Queue Multi-Server Systems}

In this chapter, the results of Section 3.2 in Chapter 3 are generalized for more general channel processes. More specifically, the stability region of multi-queue multi-server (MQMS) system is characterized for general stationary channel and arrival processes. Recall that in the system studied in Section 3.2, the channels between the queues and the servers were assumed to be stationary binary channels (i.e., $C_{n, k}(t) \in\{0,1\}$ ). For the system considered in this chapter, we assume that the channels are $M$-ary. We model the channels between the queues and the servers by a set of random variables with the common sample space $\mathcal{M}=\{0,1, \ldots, M\}$, i.e., $C_{n, k}(t) \in \mathcal{M}$. When $C_{n, k}(t)=$ $m \in \mathcal{M}$, server $k$ is capable of serving $m$ packets from queue $n$ at time slot $t$. This assumption is a valid assumption since in realistic wireless systems depending on the instantaneous SINR values, the number of packets that can be transmitted successfully over a wireless link is varying across time slots ${ }^{1}$. We also assume that the system channel process defined by matrix $C(t)=\left(C_{n, k}(t), \forall n \in \mathcal{N}, k \in \mathcal{K}\right)$ has a stationary distribution.

For such a system, the necessary and sufficient conditions for the stability of the system are derived for general arrival processes with finite first and second moments. For stationary arrival processes, these conditions establish the network stability region

${ }^{1}$ This is the common assumption when modeling a wireless channel as a finite state Markov channel. In such a modeling, depending on the system parameters (fading model, mobility pattern, power, modulation, etc.) usually a set of threshold SINR values are defined that determine the number of successful packet transmissions over the wireless link. These subjects are out of the scope of this thesis. 
of the system. Our methodology to derive the necessary conditions is similar to what we applied in the proof of Lemma 3.9 in Chapter 3. Our contribution in this chapter is to characterize the stability region as a convex polytope specified by a finite set of linear inequalities that can be numerically tabulated and used to solve network optimization problems similar to (2.10) (Refer to Lemmas 4.2 to 4.6 and Theorems 4.1 and 4.2). Furthermore, an upper bound, in an explicit form, is derived for the average queue occupancy of Maximum Weight (MW) policy [13] which is a throughput-optimal server allocation policy for MQMS system. We also study the stability of fluid model MQMS system for which using the same approach as what we use in the packetized system, the linear algebraic representation of the stability region is determined. It will be shown that the stability region in this case is characterized by an infinite number of linear inequalities. However, by an example it is shown that depending on the channel distribution and the dimension of the system, the stability region may be characterized by a limited number of non-linear inequalities instead of infinite number of linear inequalities. The contents of this chapter require some mathematical background about the fundamental concepts of polytopes and also Lyapunov stability techniques. Therefore, the reader is encouraged to consult with Appendix A if needed. We will start our discussion with the detailed description of the system in the following section.

\subsection{System Description}

Consider a time-slotted queueing system with equal-length time slots and equal-length packets. The model consists of a set of parallel queues $\mathcal{N}=\{1,2, \ldots, N\}$ and a set of identical servers $\mathcal{K}=\{1,2, \ldots, K\}$. Each server can serve at most one queue at each time slot, i.e., server sharing is not allowed in the system. At each time slot $t$, the capacity of the link between each queue $n \in \mathcal{N}$ and server $k \in \mathcal{K}$ is assumed to be $C_{n, k}(t)$ packets/time slot (Figure 4.1), i.e., during time slot $t$, server $k$ can serve at most $C_{n, k}(t)$ packets of queue $n$ successfully if it is allocated to queue $n$ at that time slot. Assume that $C_{n, k}(t) \in \mathcal{M}$ where $\mathcal{M}=\left\{m \in \mathbb{Z}_{+} \mid m \leq M\right\}$, for a given $M$. Therefore, at each time slot $t$, the channel state may be expressed by an $N \times K$ matrix $C(t)=\left(C_{n, k}(t), n \in \mathcal{N}, k \in \mathcal{K}, C_{n, k}(t) \in \mathcal{M}\right)$. The channel process is defined as $\{C(t)\}_{t=1}^{\infty}$ with the state space $\mathcal{S}$. Note that $\mathcal{S}$ is a finite set with $|\mathcal{S}|=(M+1)^{N K}$. Each element of $\mathcal{S}$ is labeled by a positive integer index $s \in\left\{1,2, \ldots,(M+1)^{N K}\right\}$. 


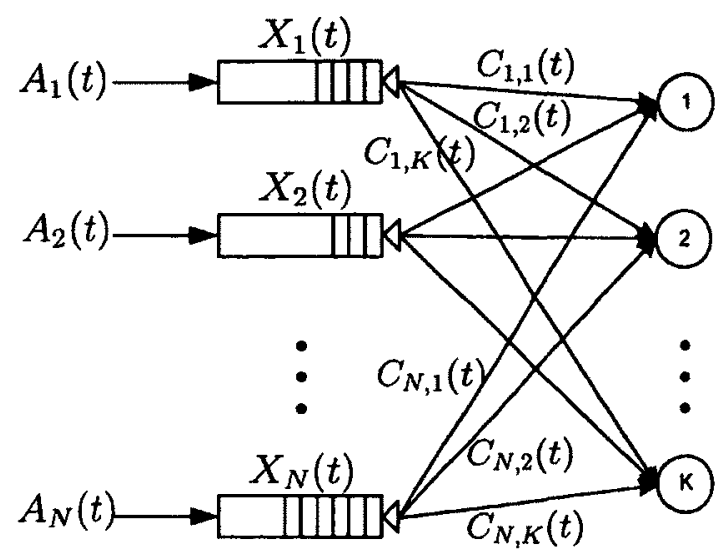

Figure 4.1: Multi-queue multi-server queueing system with stationary channel distribution

Suppose that the channel state matrix associated to the channel state $s$ is denoted by $C_{s}$. The channel process is assumed to have stationary distribution with stationary probabilities $\pi_{s}=\operatorname{Pr}\left(C(t)=C_{s}\right)$.

Queues are fed by exogenous packet arrival processes $A_{n}(t), n=1,2, \ldots, N$, i.e., the number of packet arrivals to queue $n$ during time slot $t$ is represented by $A_{n}(t)$. The arrival vector at time slot $t$ is denoted by $A(t)=\left(A_{1}(t), A_{2}(t), \ldots, A_{N}(t)\right)$. For these processes, suppose that $E\left[A_{n}^{2}(t)\right] \leq A_{\max }^{2}<\infty$ for all $t$. Assume that each queue has an infinite buffer space. The new arrivals are assumed to be added to each queue at the end of each time slot. Let $X(t)=\left(X_{1}(t), \ldots, X_{N}(t)\right)$ be the queue length vector at the end of time slot $t$ after adding new arrivals to the queues ${ }^{2}$.

A server scheduling policy at each time slot should decide how to allocate servers from set $\mathcal{K}$ to the queues in set $\mathcal{N}$. This must be accomplished based on the available information about the channel state of the system at time slot $t$ (i.e., $C(t)$ ) and also the queue length state at the beginning of time slot $t$ (i.e., $X(t-1)$ ). Therefore, at the beginning of each time slot $t$ the scheduler has to determine an allocation matrix $I(t) \in \mathcal{I}$ where

$$
\mathcal{I}=\left\{I_{N \times K}=\left(I_{n, k}, I_{n, k} \in\{0,1\}\right) \mid \sum_{n=1}^{N} I_{n, k} \leq 1 \forall k \in \mathcal{K}\right\}
$$

$\mathcal{I}$ is called allocation matrix space. Note that each matrix in $\mathcal{I}$ can have at most a

\footnotetext{
${ }^{2}$ During each time slot, departures occur first and then at the end of the time slot new arrivals are added to the queues.
} 
single " 1 " in each of its columns. The queue length vector evolves with time according to the following rule.

$$
X^{\mathrm{T}}(t)=\left(X^{\mathrm{T}}(t-1)-(C(t) \circledast I(t)) \underline{1}_{K}^{\mathrm{T}}\right)^{+}+A^{\mathrm{T}}(t)
$$

where $(\cdot)^{+}$is defined as follows: For an arbitrary vector $v$ of size $|v|,(v)^{+}$is a vector of the same size whose $i^{\prime}$ th element, $(v)_{i}^{+}$, is defined by

$$
(v)_{i}^{+}= \begin{cases}0, & \text { if } v_{i}<0 \\ v_{i}, & \text { if } v_{i} \geq 0\end{cases}
$$

\subsection{Linear Algebraic Representation of Stability Re- gion Ploytope}

In this section, we first introduce the convex hull representation of the achievable service rates (See Sections 2.2 .2 and 3.3.2). Then, the necessary and sufficient conditions for the stability of MQMS system with stationary channel processes and general arrival processes are derived. It will be shown that for stationary packet arrival processes, these conditions establish the stability region polytope. We determine a linear algebraic representation of the stability region in which we explicitly determine the coefficients of the linear inequalities describing the stability region polytope.

\subsubsection{Region of Achievable Service Rates}

Consider the class of deterministic policies $\mathcal{G}$ where at each state of the system, each policy $g \in \mathcal{G}$ allocates the servers according to a predetermined allocation matrix depending on the channel state matrix (i.e., matrix $C(t)$ ). More specifically, for each policy $g \in \mathcal{G}$, there exists a one-to-one mapping from the channel state space $\mathcal{S}$ to the allocation matrix space $\mathcal{I}$, namely $I^{(g)}: \mathcal{S} \longmapsto \mathcal{I}$. Therefore, each deterministic policy $g$ is specified by $|\mathcal{S}|$ allocation matrices $I_{s}^{(g)}, s \in \mathcal{S}$. The scheduler observes the state of the system and then based on the observed state $s$, it allocates the servers according to an allocation matrix $I_{s}^{(g)}$. Note that set $\mathcal{I}$ is a finite set and since the channel state space is also finite, set $\mathcal{G}$ is finite.

Each deterministic policy $g$ provides an average service rate for each queue $n$. 
Let $R_{n}^{(g)}$ denote the time averaged service rate provided to queue $n$ and $R^{(g)}=$ $\left(R_{1}^{(g)}, R_{2}^{(g)}, \ldots, R_{N}^{(g)}\right)$ the vector of average service rates. By conditioning on the channel state of the system, it is not hard to see that for each deterministic server allocation policy $g$, we have

$$
R^{(g)}=\left(\sum_{s \in \mathcal{S}} \pi_{s}\left(C_{s} \circledast I_{s}^{(g)}\right) \underline{1}_{K}^{\mathrm{T}}\right)^{\mathrm{T}}
$$

Note that each rate vector $R^{(g)}$ determines a single point in $\mathbb{R}_{+}^{N}$. Now, consider the convex hull of all the points $R^{(g)}, g \in \mathcal{G}$ in $\mathbb{R}_{+}^{N}$, i.e.,

$$
\mathcal{R}=\underset{g \in \mathcal{G}}{\operatorname{conv}} \operatorname{hull}\left(R^{(g)}\right)
$$

Each point in $\mathcal{R}$, let say $R^{\star}$ can be represented by a convex combination of a finite set of points, $R^{(g)}, g \in \mathcal{G}$, i.e.,

$$
R^{\star}=\sum_{i=1}^{|\mathcal{G}|} p_{i} R^{g_{i}}, g_{i} \in \mathcal{G}, \sum_{i=1}^{|\mathcal{G}|} p_{i}=1, p_{i} \geq 0
$$

Hence, $\mathcal{R}$ contains all the achievable service rate vectors of MQMS system. To achieve the service rate vector $R^{\star} \in \mathcal{R}$ it is enough to select policy $g_{i}$ with probability $p_{i}$, i.e., in $p_{i}$ fraction of time slots. In other words, all the service rate vectors $R^{\star} \in \mathcal{R}$ are achievable by applying a randomized policy that at each time slot selects policy $g_{i}$ with probability $p_{i}$. Thus, the following lemma follows.

Lemma 4.1 The set of achievable service rate vectors $\mathcal{R}$ is specified by a polytope.

Proof: The lemma follows directly from the definition of polytope in (A.1), equation (4.4) and the fact that set $\mathcal{G}$ is finite.

Henceforth, we will denote the achievable service rate polytope by $\mathcal{P}$.

\subsubsection{Necessary Conditions for the Stability of MQMS System}

We first give an outline of the steps that lead us to find the necessary conditions for the stability of the system.

- Using Lemmas 4.2-4.4, all the face-defining hyperplanes (Refer to Appendix A.2 for the definition of face and facet) of the stability region polytope are 
characterized. More specifically, these lemmas determine the normal vector associated to each hyperplane as well as a single point where the hyperplane touches the stability region polytope. It will be shown that each vector in $\mathbb{R}_{+}^{N}$ is associated to a face-defining hyperplane.

- The stability region is a polytope and therefore can be characterized by the finite set of its facet-defining hyperplanes. Lemma 4.5 introduces a subset of $\mathbb{R}_{+}^{N}$, namely $V \subset \mathbb{R}_{+}^{N}$ and proves that the vectors outside set $V$ cannot be associated to a facet-defining hyperplanes of the stability region polytope. Note that set $V$ is an infinite set itself.

- Lemma 4.6 shows that although set $V$ is an infinite set, it is finite up to multiplication of vectors by positive scalars, i.e., all the vectors in $V$ can be produced by multiplying a positive scalar to a vector in a finite set $\widehat{V}$.

- Using Lemmas 4.2 to 4.6 , Theorem 4.1 is proven which states the necessary conditions for the stability of the system. We argue that the derivation of set $\widehat{V}$ becomes difficult for large $N$ and $M$. In this case, a finite superset of set $\widehat{V}$ is introduced whose elements can be specified easily.

Consider the departure matrix $H_{N \times K}(t)=\left(H_{n, k}(t), n \in \mathcal{N}, k \in \mathcal{K}\right)$ in which $H_{n, k}(t)\left(H_{n, k}(t) \leq C_{n, k}(t)\right)$ represents the total number of packets served by server $k$ from queue $n$ at time slot $t$. The departure process from queue $n$ at time slot $t$ would be $\sum_{k=1}^{K} H_{n, k}(t)$. Thus, the following equality illustrates the evolution of queue length process in time.

$$
X_{n}(t)=X_{n}(t-1)-\sum_{k=1}^{K} H_{n, k}(t)+A_{n}(t) \quad \forall n \in \mathcal{N}
$$

For a strongly stable MQMS queueing system, the following lemma is true. This lemma is similar to Lemma 3.7 proven in Chapter 3 for multi-server single-interface queueing system with random connectivities.

Lemma 4.2 If the MQMS system is strongly stable, then for any vector $\alpha=$ $\left(\alpha_{1}, \alpha_{2}, \ldots, \alpha_{N}\right) \in \mathbb{R}^{N}$ we have

$$
\overline{\alpha E[A(t)]^{\mathrm{T}}}=\overline{\alpha E\left[H(t) \underline{1}_{K}^{\mathrm{T}}\right.}
$$


The proof is similar to the proof of Lemma 3.7 in Chapter 3 and is skipped here. A direct result of Lemma 4.2 for a single queue $n$ is the following.

$$
\overline{E\left[A_{n}(t)\right]}=\overline{E\left[\sum_{k=1}^{K} H_{n, k}(t)\right]}
$$

In other words, for a strongly stable queue $n$, the time averaged total expected arrival to queue $n$ is equal to the time averaged total expected departure from queue $n$.

Lemma 4.3 If the MQMS system is strongly stable, then $\overline{E[A(t)]} \in \mathcal{P}$.

Proof: The proof follows directly by considering (4.8) and the fact that

$$
\forall H(t), \exists R \in \mathcal{P}: \overline{E\left[\sum_{k=1}^{K} H_{n, k}(t)\right]} \leq R_{n} \quad \forall n \in \mathcal{N}
$$

Based on Lemmas 4.2 and 4.3 , the following lemma is proven.

Lemma 4.4 If the MQMS system is strongly stable, then for all $\alpha \in \mathbb{R}_{+}^{N}$

$$
\alpha \overline{E[A(t)]^{\mathrm{T}}} \leq \sum_{s \in \mathcal{S}} \pi_{s} \max _{I \in \mathcal{I}}\left(\alpha\left(C_{s} \circledast I\right) \underline{1}_{K}^{\mathrm{T}}\right)
$$

Proof: Consider $\alpha \in \mathbb{R}^{N}$. Since the system is strongly stable, from Lemma 4.2 we have

$$
\lim _{t \rightarrow \infty} \frac{1}{t} \sum_{\tau=1}^{t} \alpha E[A(\tau)]^{\mathrm{T}}=\lim _{t \rightarrow \infty} \frac{1}{t} \sum_{\tau=1}^{t} \alpha E[H(\tau)] \underline{1}_{K}^{\mathrm{T}}
$$


By conditioning on the channel state process, we will have

$$
\begin{aligned}
\lim _{t \rightarrow \infty} & \frac{1}{t} \sum_{\tau=1}^{t} \alpha E[H(\tau)] \underline{1}_{K}^{\mathrm{T}} \\
& =\lim _{t \rightarrow \infty} \frac{1}{t} \sum_{\tau=1}^{t} \sum_{s \in \mathcal{S}} \pi_{s} \alpha E\left[H(\tau) \mid S(\tau)=s \underline{1}_{K}^{\mathrm{T}}\right. \\
& \leq \lim _{t \rightarrow \infty} \frac{1}{t} \sum_{\tau=1}^{t} \sum_{s \in \mathcal{S}} \pi_{s}(\alpha)^{+} E\left[C_{s} \circledast I(\tau)\right] \underline{1}_{K}^{\mathrm{T}} \\
& \leq \lim _{t \rightarrow \infty} \frac{1}{t} \sum_{\tau=1}^{t} \sum_{s \in \mathcal{S}} \pi_{s} \max _{I \in \mathcal{I}}\left((\alpha)^{+}\left(C_{s} \circledast I\right) \underline{1}_{K}^{\mathrm{T}}\right) \\
& =\sum_{s \in \mathcal{S}} \pi_{s} \max _{I \in \mathcal{I}}\left((\alpha)^{+}\left(C_{s} \circledast I\right) \underline{1}_{K}^{\mathrm{T}}\right)
\end{aligned}
$$

Now, consider two different cases:

- $\alpha \in \mathbb{R}_{+}^{N}$ : The result follows directly from (4.11) and (4.12) since $(\alpha)^{+}=\alpha$.

- $\alpha \notin \mathbb{R}_{+}^{N}$ : In this case, from (4.11) and (4.12) we have the following inequality.

$$
\alpha \overline{E[A(t)]^{\mathrm{T}}} \leq \sum_{s \in \mathcal{S}} \pi_{s} \max _{I \in \mathcal{I}}\left((\alpha)^{+}\left(C_{s} \circledast I\right) \underline{1}_{K}^{\mathrm{T}}\right)
$$

However, since $(\alpha)^{+} \in \mathbb{R}_{+}^{N}$ and according to the previous case we also have

$$
(\alpha)^{+} \overline{E[A(t)]^{\mathrm{T}}} \leq \sum_{s \in \mathcal{S}} \pi_{s} \max _{I \in \mathcal{I}}\left((\alpha)^{+}\left(C_{s} \circledast I\right) \underline{1}_{K}^{\mathrm{T}}\right)
$$

Noting (4.14) and the fact that $E[A(t)] \in \mathbb{R}_{+}^{N}$, we conclude that (4.13) is a redundant inequality for polytope $\mathcal{P}$ (Refer to Appendix A.2 for the definition of redundant inequality).

Since $\alpha \in \mathbb{R}_{+}^{N}$, the set of inequalities in (4.10) forms an infinite set. Each inequality in (4.10) determines a valid inequality for polytope $\mathcal{P}$ (Refer to Appendix A.2 for the definition of valid inequality).

Fact: The hyperplanes associated to the valid inequalities of $(4.10)$ are all facedefining hyperplanes of polytope $\mathcal{P}$. 
To show this fact, let $\mathcal{I}^{\alpha}$ denote a set of allocation matrices $\left\{I_{s}^{\alpha}, s \in \mathcal{S}\right\}$ that maximize the right hand side of (4.10), i.e.,

$$
I_{s}^{\alpha}=\arg \max _{I \in \mathcal{I}} \alpha\left(C_{s} \circledast I\right) \underline{1}_{K}^{\mathrm{T}}
$$

$\mathcal{I}^{\alpha}$ is not unique and there may be more than one set of allocation matrices of $\mathcal{I}^{\alpha}$ whose elements maximize $\alpha\left(C_{s} \circledast I\right) \underline{1}_{K}^{\mathrm{T}}$. According to (4.3) and (4.4), $\left(\sum_{s \in \mathcal{S}} \pi_{s}\left(C_{s} \circledast I_{s}^{\alpha}\right) \underline{1}_{K}^{\mathrm{T}}\right)^{\mathrm{T}} \in \mathcal{P}$. On the other hand, since $\sum_{s \in \mathcal{S}} \pi_{s} \alpha\left(C_{s} \circledast I_{s}^{\alpha}\right) \underline{1}_{K}^{\mathrm{T}}=$ $\alpha \sum_{s \in \mathcal{S}} \pi_{s}\left(C_{s} \circledast I_{s}^{\alpha}\right) \underline{1}_{K}^{\mathrm{T}}$, point $\sum_{s \in \mathcal{S}} \pi_{s}\left(C_{s} \circledast I_{s}^{\alpha}\right) \underline{1}_{K}^{\mathrm{T}}$ is located on the hyperplane associated to (4.10). Therefore, the set of inequalities in (4.10) determines all the non-empty faces of polytope $\mathcal{P}$.

According to the redundancy theorem in polytopes (Refer to Section A.2), not all the face-defining hyperplanes of a polytope are required to describe a polytope. In particular, just the inequalities corresponding to the facets of a polytope are sufficient to characterize a polytope. In the following, the facets of polytope $\mathcal{P}$ are characterized. The analysis follows and constitutes the main contribution in this chapter.

Let $V$ denote the set of vectors $\alpha \in \mathbb{R}_{+}^{N}$ with the following property, i.e.,

$$
\begin{aligned}
V:= & \left\{\alpha \in \mathbb{R}_{+}^{N} \mid \forall\left(\mathcal{U} \subset \mathcal{N}, \mathcal{U} \neq \emptyset, \alpha_{\mathcal{U}} \neq \underline{0}_{|\mathcal{U}|}, \alpha_{\mathcal{U}^{c}} \neq \underline{0}_{|\mathcal{U} c|}\right)\right. \\
& \left.\quad \exists\left(i \in \mathcal{U}, j \in \mathcal{U}^{c}, m, n \in \mathcal{M}, \alpha_{i}, \alpha_{j}, m, n \neq 0\right): \alpha_{i} m=\alpha_{j} n\right\} .
\end{aligned}
$$

A vector $\alpha$ belongs to set $V$ if for any partitioning of elements of vector $\alpha$ into two nonempty disjoint (sub) vectors in which all the elements of each vector are not identically equal to zero, there exists at least one non-zero element in each (sub)vector, the ratio of which is equal to the ratio of two non-zero elements of set $\mathcal{M}$. To clarify the definition of set $V$ consider the following example.

Example: Let $\mathcal{M}=\{0,1,2\}$ and $N=4$. Now, consider vector $\alpha_{1}=(1,2,5,10)$. We can partition $\alpha_{1}$ to $\alpha_{1_{\{1,2\}}}=(1,2)$ and $\alpha_{1_{\{3,4\}}}=(5,10)$. Note that there exist no two elements one in $\alpha_{1_{\{1,2\}}}$ and the other in $\alpha_{1_{\{3,4\}}}$ whose ratio is 1 or 2 and therefore, $\alpha_{1} \notin V$. Another example is vector $\alpha_{2}=(0,2.5,0,0)$ in which for any partitioning of the vector into two non-empty disjoint (sub) vectors one will always have all the elements identically equal to zero. Therefore, $\alpha_{2} \in V$.

In the following, it will be shown that any vector in $\mathbb{R}_{+}^{N}-V$ cannot form a facet-defining hyperplane for polytope $\mathcal{P}$ and therefore the inequalities derived by the vectors of set $\mathbb{R}_{+}^{N}-V$ are redundant. This is shown in the following lemma. 
Lemma 4.5 If $\alpha \in \mathbb{R}_{+}^{N}-V$, the hyperplane associated to the valid inequality of (4.10) is not a facet-defining hyperplane of $\mathcal{P}$.

The proof is presented in Appendix C.1.

Note that $V$ is an infinite set. However, we can show that set $V$ is finite up to multiplication of vectors by positive scalars. To show this, we define set $\mathcal{W}=$ $\left\{z \in \mathbb{Z}_{+} \mid z=\prod_{j=1}^{N-1} m_{j}, m_{j} \in \mathcal{M}\right\}$ and $\mathcal{W}^{N}=\left\{\left(\alpha_{1}, \alpha_{2}, \ldots, \alpha_{N}\right) \mid \alpha_{n} \in \mathcal{W}\right\}$. Then, we can prove the following lemma.

Lemma 4.6 There exists $\widehat{V} \subseteq \mathcal{W}^{N}$ such that any vector $\alpha \in V$ can be written as $\alpha=q \beta$ for some vector $\beta \in \widehat{V}$ and scalar $q>0$.

The proof is given in Appendix C.2.

Example: Recall the two-queue single-server example we mentioned several times in this thesis (see Figure 3.3 in Chapter 3). Using Lemmas 4.5 and 4.6, we can characterize a finite subset of $\mathbb{R}_{+}^{2}$ that can produce all the normal vectors of the facet-defining hyperplanes of the stability region polytope for this system. For the aforementioned example, since $M=1$, we have $\mathcal{W}=\{0,1\}$ and therefore $\mathcal{W}^{2}=\{(0,0),(0,1),(1,0),(1,1)\}$. Hence, $\widehat{V} \subseteq\{(0,1),(1,0),(1,1)\}$. For this simple example, since $M=1$ (as we will see in Corollary 4.2 below), we have $\widehat{V}=\mathcal{W}^{N}-\left\{\underline{0}_{N}\right\}$. The facet-defining hyperplanes of the stability region for this example as described by the vectors in the set $\widehat{V}$ are shown with bold red lines in Figure 3.4 (in Chapter 3).

Fact: The size of $\mathcal{W}$ is $|\mathcal{W}|=\left(\begin{array}{c}M+N-2 \\ N-1\end{array}\right)+1$.

In order to prove this fact, note that $\mathcal{M}$ has $M$ non-zero elements. Each non-zero element of $\mathcal{W}$ comes from the multiplication of $N-1$ non-zero elements of $\mathcal{M}$. This is equivalent to the counting problem of choosing $N-1$ balls from $M$ distinctly marked balls without ordering and with replacement which is equal to $|\mathcal{W}|=\left(\begin{array}{c}M+N-2 \\ N-1\end{array}\right)$ [88]. Since $0 \in \mathcal{W}$ we must increase this number by 1 . Hence, $\left|\mathcal{W}^{N}\right|=\left(\left(\begin{array}{c}M+N-2 \\ N-1\end{array}\right)+1\right)^{N}$. Since $\widehat{V} \subseteq \mathcal{W}^{N}-\left\{\underline{0}_{N}\right\}$, therefore $|\widehat{V}| \leq\left|\mathcal{W}^{N}\right|-1$ (excluding $\underline{0}_{N}$ as it results into the obvious equality $0=0$ ).

In the theorem that follows, we introduce the necessary conditions for the stability of MQMS system with stationary channel distribution.

Theorem 4.1 If there exists a server allocation policy under which the system is 
stable, then

$$
\alpha \overline{E[A(t)]^{\mathrm{T}}} \leq \sum_{s \in \mathcal{S}} \pi_{s} \max _{I \in \mathcal{I}}\left(\alpha\left(C_{s} \circledast I\right) \underline{1}_{K}^{\mathrm{T}}\right), \quad \alpha \in \widehat{V} .
$$

Proof: The proof follows directly from Lemmas 4.4 to 4.6. In fact, from Lemma 4.4 we can characterize polytope $\mathcal{P}$ by an infinite number of inequalities $\left(\alpha \in \mathbb{R}_{+}^{N}\right)$. In Lemmas 4.5 and 4.6 we showed that not all of such $\alpha$ vectors are necessary to describe the facets of polytope $\mathcal{P}$. More specifically, we proved that just $\alpha \in \widehat{V}$ $(|\widehat{V}|<\infty)$ are sufficient to describe the facets of polytope $\mathcal{P}$ and other $\alpha$ vectors make redundant inequalities and therefore the theorem follows.

According to Theorem 4.1, in order to characterize polytope $\mathcal{P}$, we have to specify all the elements of set $\widehat{V}$ which can be obtained after considering all the possible vectors $\alpha \in \mathcal{W}^{N}-\left\{\underline{0}_{N}\right\}$ and removing redundancies following (4.16). This can be achieved numerically. We may also avoid the numerical computation to derive set $\widehat{V}$ and instead use set $\mathcal{W}^{N}-\left\{\underline{0}_{N}\right\}$ which is a finite superset of $\widehat{V}$ that contains redundancies (produces redundant inequalities). Although by using $\mathcal{W}^{N}-\left\{\underline{0}_{N}\right\}$ we may obtain some redundant inequalities, since $\left|\mathcal{W}^{N}-\left\{\underline{0}_{N}\right\}\right|<\infty$ we still have a finite number of inequalities to describe polytope $\mathcal{P}$. Table 4.1 depicts $\widehat{V}$ and $\left|\mathcal{W}^{N}\right|-1$ for some sample cases $N=2,3$ and $M=1,2,3,4$.

Table 4.1: $|\widehat{V}|$ and $\left|\mathcal{W}^{N}\right|-1$ for $N=2,3$ and $M=1,2,3,4$

\begin{tabular}{|c||c|c|c|c|c|}
\cline { 3 - 6 } \multicolumn{2}{c|}{} & $M=1$ & $M=2$ & $M=3$ & $M=4$ \\
\hline \multirow{2}{*}{$|\widehat{V}|,\left|\mathcal{W}^{N}\right|-1$} & $N=2$ & 3,3 & 5,8 & 9,15 & 12,24 \\
\cline { 2 - 6 } & $N=3$ & 7,7 & 25,63 & 109,342 & 253,1330 \\
\hline
\end{tabular}

Corollary 4.1 For an MQMS system with stationary arrival processes, we have $E[A(t)]=\lambda=\left(\lambda_{1}, \lambda_{2}, \ldots, \lambda_{N}\right)$ and the necessary conditions for the stability of the system would be

$$
\alpha \lambda^{\mathrm{T}} \leq \sum_{s \in \mathcal{S}} \pi_{s} \max _{I \in \mathcal{I}}\left(\alpha\left(C_{s} \circledast I\right) \underline{1}_{K}^{\mathrm{T}}\right), \quad \alpha \in \hat{V}
$$


Corollary 4.2 For an MQMS system with ON-OFF channels, we have $\mathcal{W}=\{0,1\}$ and therefore the necessary conditions for the stability of the system would be

$$
\alpha \overline{E[A(t)]^{\mathrm{T}}} \leq \sum_{s \in \mathcal{S}} \pi_{s} \max _{I \in \mathcal{I}}\left(\alpha\left(C_{s} \circledast I\right) \underline{1}_{K}^{\mathrm{T}}\right), \quad \alpha \in\{0,1\}^{N}-\left\{\underline{0}_{N}\right\} .
$$

In this case, the total number of inequalities needed to describe polytope $\mathcal{P}$ is equal to $2^{N}-1$.

\subsubsection{Sufficient Conditions for the Stability of MQMS System}

Recall the Maximum Weight (MW) server allocation policy described in Section 3.2.2 of Chapter 3. For the MQMS system with $M$-ary channels, this policy determines the allocation matrix at each time slot $t$ by solving the following maximization problem.

$$
I(t)=\arg \max _{I \in \mathcal{I}} X(t-1)(C(t) \circledast I) \underline{1}_{K}^{\mathrm{T}}
$$

According to the constraints on allocation matrix $I$, we can easily conclude that MW policy allocates the servers by the following rule: at each time slot $t$, each server $k$ is allocated to the queue $n$ that achieves the maximum $X_{n}(t-1) C_{n, k}(t)$.

In the following, the sufficient conditions for the stability of the stationary MQMS model is derived and it will be proven that MW stabilizes the system as long as condition (4.21) below is satisfied. An upper bound for the time averaged expected number of packets in the system was also obtained.

Theorem 4.2 The MQMS system is stable under $M W$ policy if for all $t$

$$
\alpha E[A(t)]^{\mathrm{T}}<\sum_{s \in \mathcal{S}} \pi_{s} \max _{I \in \mathcal{I}}\left(\alpha\left(C_{s} \circledast I\right) \underline{1}_{K}^{\mathrm{T}}\right), \quad \alpha \in \widehat{V} .
$$

Furthermore, the following bound for the average expected "aggregate" occupancy holds.

$$
\limsup _{t \rightarrow \infty} \frac{1}{t} \sum_{\tau=0}^{t-1} \sum_{n=1}^{N} E\left[X_{n}(\tau)\right] \leq \frac{N A_{\max }^{2}+(M K)^{2}}{2 \delta}
$$

where $\delta=\min _{t, \alpha \in \widehat{V}}\left\{\frac{1}{\sum_{i=1}^{N} \alpha_{i}}\left(\sum_{s \in \mathcal{S}} \pi_{s} \max _{I \in \mathcal{I}}\left(\alpha\left(C_{s} \circledast I\right) \underline{1}_{K}^{\mathrm{T}}\right)-\alpha E[A(t)]^{\mathrm{T}}\right)\right\}$. 
The detailed proof is given in Appendix C.3.

Corollary 4.3 For an MQMS system with stationary arrival processes, we have $E[A(t)]=\lambda$ and the sufficient conditions for the stability of the system under $M W$ policy are $\alpha \lambda^{\mathrm{T}}<\sum_{s \in \mathcal{S}} \pi_{s} \max _{I \in \mathcal{I}}\left(\alpha\left(C_{s} \circledast I\right) \underline{1}_{K}^{\mathrm{T}}\right), \quad \alpha \in \widehat{V}$.

According to Corollaries 4.1 and 4.3 and the definition of network stability region, we can conclude that for an MQMS system with stationary channel distribution and stationary arrival processes, the stability region is characterized by inequalities (4.18). The stability region described in (4.18) is a polytope which can be imagined for 2 and 3 dimensional systems, i.e., $N=2,3$. Figure 4.2 shows the stability region for $N=2,3$ and $M=1,2,3,4$. In all the cases, $K=3$.

Consider an MQMS system with ON-OFF channels. In such a system, $\mathcal{W}=\{0,1\}$ and therefore we have the following corollary.

Corollary 4.4 The MQMS system with ON-OFF channels is stable under MW policy if for all $t$

$$
\alpha E[A(t)]^{\mathrm{T}}<\sum_{s \in \mathcal{S}} \pi_{s} \max _{I \in \mathcal{I}}\left(\alpha\left(C_{s} \circledast I\right) \underline{1}_{K}^{\mathrm{T}}\right), \quad \alpha \in\{0,1\}^{N}-\left\{\underline{0}_{N}\right\}
$$

\subsubsection{Numerical Example}

In this section, a simple numerical example of MQMS system with $N=2, K=3$ and $M=3$ is considered. For such a system, the size of channel state space is $4^{6}=4096$. The channel distribution was chosen randomly. Assume that the utility functions associated to queues 1 and 2 are $\log \left(1+10 r_{1}\right)$ and $10 r_{2}$, respectively ${ }^{3}$. Therefore the total utility function is $f\left(r_{1}, r_{2}\right)=\log \left(1+10 r_{1}\right)+10 r_{2}$. The packet arrival processes to queues 1 and 2 are Poisson distributed, with rate 5 packets/time slot. First, we are going to characterize the stability region for this example using the theorems given in the previous subsections. Then, we will correlate our findings with the results from $[1,16,23]$.

For the described system we can easily check that $\mathcal{W}=\{0,1,2,3\}$. Thus, $\mathcal{W}^{2}-\{(0,0)\}=\{(0,1),(0,2),(0,3),(1,0),(1,1),(1,2),(1,3),(2,0),(2,1),(2,2),(2,3)$, $(3,0),(3,1),(3,2),(3,3)\}$ whose size is 15 (as mentioned in Table 4.1). Note that

\footnotetext{
${ }^{3}$ Linear and logarithmic utility functions were studied in $[1,16]$.
} 


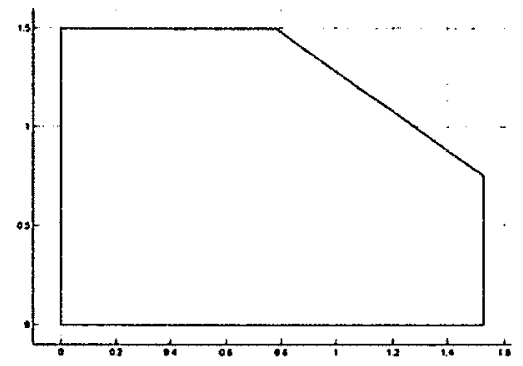

(a) $N=2, M=1$

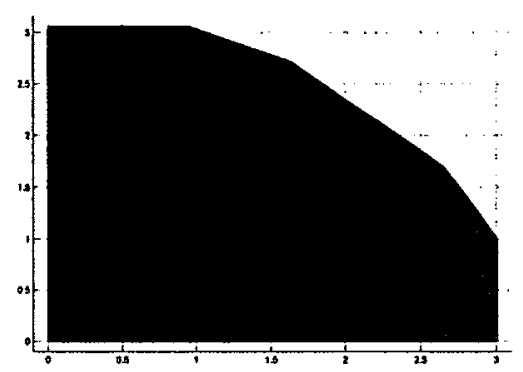

(c) $N=2, M=2$

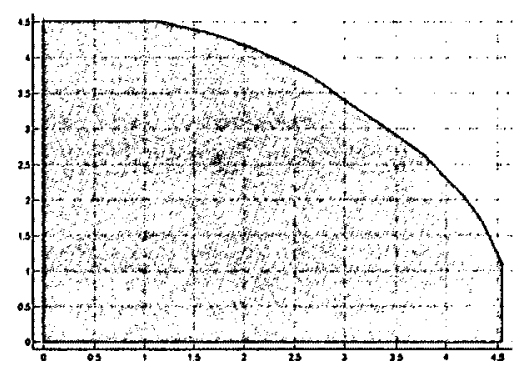

(e) $N=2, M=3$

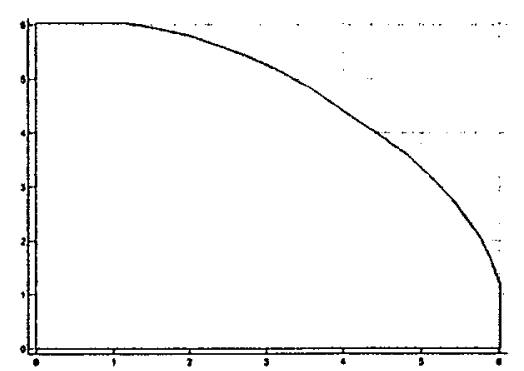

(g) $N=2, M=4$

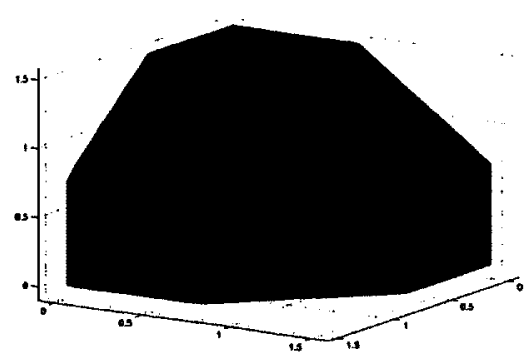

(b) $N=3, M=1$

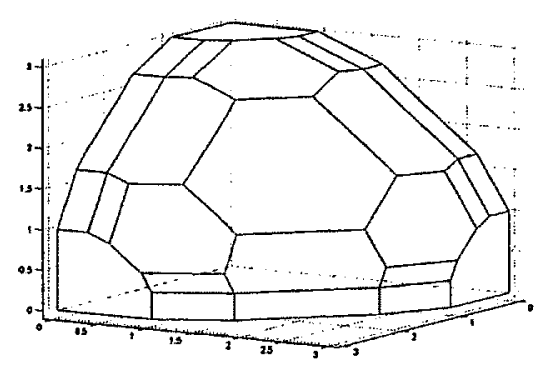

(d) $N=3, M=2$

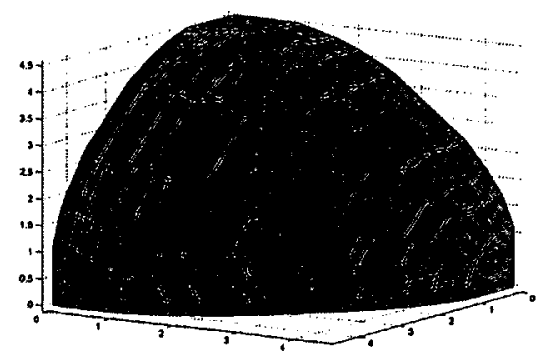

(f) $N=3, M=3$

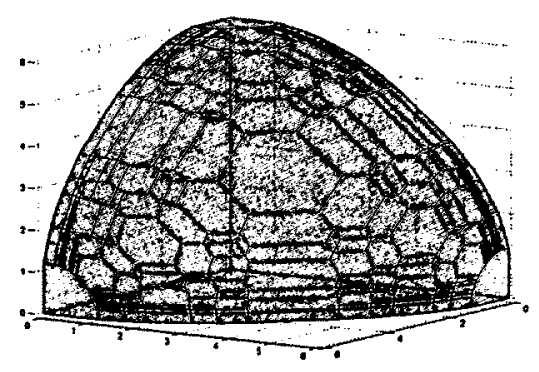

(h) $N=3, M=4$

Figure 4.2: Stability region for $N=2,3, M=1,2,3,4$ and $K=3$ 
some of these vectors result in redundant inequalities. For example using $(1,1)$, $(2,2)$ and $(3,3)$ as the vector $\alpha$ in $(4.18)$ will result in the same inequalities. By removing the redundant vectors, we obtain set $\widehat{V}=\{(0,1),(1,0),(1,1),(1,2)$, $(1,3),(2,1),(2,3),(3,1),(3,2)\}$. Using $(4.18)$, the stability region is characterized by the following set of inequalities which is also shown in Figure 4.3.

$$
\begin{array}{rlr}
r_{1} \leq 4.4792 & r_{1}+2 r_{2} \leq 10.2893 & 2 r_{1}+3 r_{2} \leq 16.4564 \\
r_{2} \leq 4.4912 & r_{1}+3 r_{2} \leq 14.6002 & 3 r_{1}+r_{2} \leq 14.5803 \\
r_{1}+r_{2} \leq 6.3577 & 2 r_{1}+r_{2} \leq 10.2874 & 3 r_{1}+2 r_{2} \leq 16.4639
\end{array}
$$

Therefore, the utility optimization problem is specified as

$$
\text { Maximize: } \quad \log \left(1+10 r_{1}\right)+10 r_{2}
$$

Subject to: All the constraints in (4.24)

$$
0 \leq r_{1} \leq 5 \quad, \quad 0 \leq r_{2} \leq 5
$$

The solution to the above problem is $r^{\star}=\left(r_{1}^{\star}, r_{2}^{\star}\right)=(1.1266,4.4912)$ and is indicated by the star in bold in Figure 4.3. As we expect the optimal point is located on the boundary of the stability region. For each queue, we can use a leaky bucket and adjust the admitted rate to each queue to the optimal value we obtained from the optimization problem.

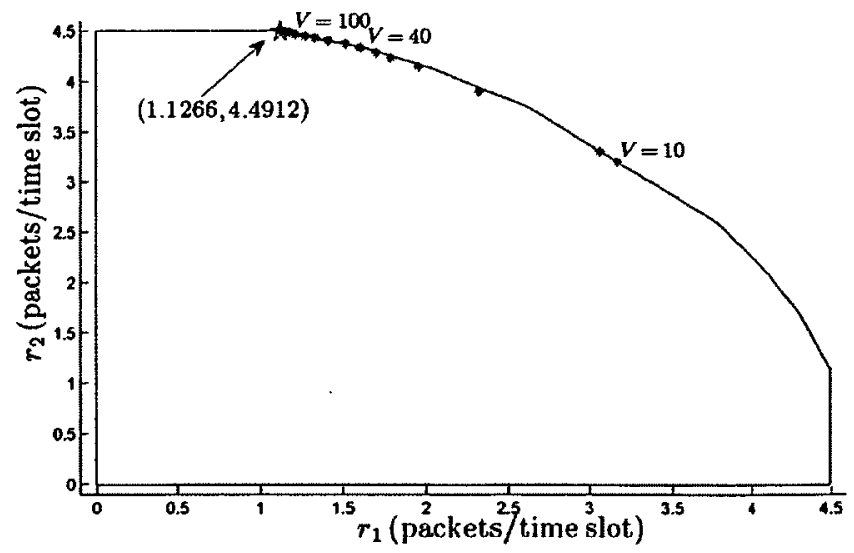

Figure 4.3: Stability region for $N=2, K=3, M=3$ with a random channel distribution (the optimal solution and the solution of CLC2b algorithm are also depicted in this figure) 
We have also simulated the flow control strategy CLC2b (Cross-Layer Control 2-b) which was introduced in $[1,16,23]$ (The reader is suggested to refer to the literature regarding this algorithm). CLC2b was proposed as a flow control strategy for a general queueing system for which the stability region is unknown. The algorithm assumes that $A_{n}(t) \leq R_{n}^{\max }$ for all $t$. Assume that the backlog in the transport layer of queue $n$ at the beginning of time slot $t$ is denoted by $L_{n}(t)$. CLC2b algorithm is a dynamic flow control algorithm in the transport layer in which at each time slot it determines how many packets to admit to queue $n$ (in the network layer) from the transport layer (i.e., from $L_{n}(t)$ packets in the transport layer waiting for admission). Assume that this number is denoted by $r_{n}(t)$. Therefore, the goal of CLC2b algorithm is to determine $r_{n}(t)$ such that $f(\bar{r})$ is maximized while the queues are kept stable. CLC2b algorithm incorporates two novel notions, namely auxiliary variables and virtual cost queues. In the following, they are briefly reviewed.

The utility optimization in (2.10) can be transformed into the following stochastic optimization problem by introducing the auxiliary variables $\gamma_{n}, n \in \mathcal{N}[1,16,23]$.

$$
\begin{array}{cl}
\underset{\gamma, r}{\operatorname{Maximize}:} & \sum_{n=1}^{N} f_{n}\left(\gamma_{n}\right) \\
\text { Subject to: } & r_{n} \geq \gamma_{n} \\
& r=\left(r_{1}, r_{2}, \ldots, r_{N}\right) \in \Lambda \\
& 0 \leq r_{n} \leq \lambda_{n} \quad \forall n=1,2, \ldots, N .
\end{array}
$$

For each queue $n$ a virtual cost queue with virtual arrival process $\gamma_{n}(t)$ and virtual service process $r_{n}(t)$ is defined (See Figure 4.4 and refer to [1]). Note that the service process of the virtual queue $n$ is exactly equal to the admitted packet process to the actual queue $n$. Assuming that the length of this virtual queue is denoted by $Y_{n}(t)$ at time slot $t$, the following equation reveals the evolution of this process by time.

$$
Y_{n}(t)=\left(Y_{n}(t-1)-r_{n}(t)\right)^{+}+\gamma_{n}(t)
$$

It was shown in $[1,16]$ that if a control strategy stabilizes both the actual and virtual queues then the resulting averages $\bar{r}_{n}$ and $\bar{\gamma}_{n}$ will satisfy the constraints of the optimization problem (4.26). The flow control strategy of CLC2b is specified as follows.

- Every timeslot and for each queue $n$ observe $X_{n}(t-1)$ and $Y_{n}(t-1)$ and 


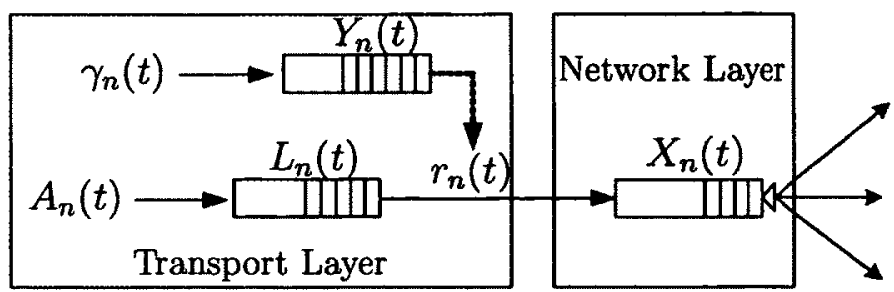

Figure 4.4: CLC2b algorithm framework

determine

$$
r_{n}(t)=\left\{\begin{array}{cc}
\min \left\{L_{n}(t)+A_{n}(t), R_{n}^{\max }\right\}, & \text { if } \eta Y_{n}(t-1)>X_{n}(t-1) \\
0, & \text { otherwise }
\end{array}\right.
$$

- Choose $\gamma_{n}(t)$ (the input process of queue $n$ 's virtual queue) as the solution of the following optimization problem and update $Y_{n}(t)$ following (4.27).

$$
\begin{array}{cl}
\underset{\gamma}{\operatorname{Maximize}:} & V f_{n}(\gamma)-\eta Y_{n}(t-1) \gamma \\
\text { Subject to: } & 0 \leq \gamma \leq R_{n}^{\max } .
\end{array}
$$

- Use the MW policy for resource (server) allocation and update $X_{n}(t)$.

$f_{n}(\cdot)$ is the utility function associated to queue $n$ and $\eta$ is a positive weight that satisfies $0<\eta \leq 1$. It determines the relative weight of the virtual queue in stabilizing the system. $V$ is a control parameter that affects the proximity of $\bar{r}_{n}$ (the solution of $\mathrm{CLC} 2 \mathrm{~b}$ algorithm) to the optimal point $r^{\star}$. It was shown that CLC2b can stabilize both the actual and virtual queues while guaranteeing a lower bound for the achieved utility function (given by (4.30)) and also an upper bound for the system backlog in the network layer (given by (4.31)).

$$
\begin{gathered}
\liminf _{t \rightarrow \infty} f(\bar{r}) \geq f\left(r^{\star}\right)-\frac{D}{V} \\
\limsup _{t \rightarrow \infty} \frac{1}{t} \sum_{\tau=0}^{t} \sum_{n=1}^{N} X_{n}(\tau) \leq \frac{D+V G}{\mu}
\end{gathered}
$$

Where $D, G$ and $\mu$ are positive constants and are dependent to the statistical properties of the system. We can observe that as the control parameter $V$ increases, the 
difference between $f(\bar{r})$ and the optimal utility $f\left(r^{\star}\right)$ decreases as $\frac{1}{V}$. However, the closer $f(\bar{r})$ and $f\left(r^{\star}\right)$ are, the larger the backlog is in the system!

The CLC2b algorithm was simulated with $\eta=1$. Furthermore, a simulation was performed where the admitted rates to the queues are controlled by leaky buckets outputting the optimal rates $r^{\star}=(1.1266,4.4912)$ as determined by our analysis earlier. The two systems were compared by computing the following measures versus $V$.

- Difference of the average queue occupancy of the systems, i.e., $\overline{X_{C L C 2 b}}-\overline{X_{o p t}}$ shown in Figure 4.5(a).

- Percentage of the normalized difference of the utility function for $\bar{r}$ and $r^{\star}$, i.e., $\frac{f\left(r^{\star}\right)-f\left(\bar{r}_{C L C 2 b}\right)}{f\left(r^{\star}\right)} \times 100$ shown in Figure 4.5(b).

We can see from the graphs that the queue occupancy under CLC2b algorithm is growing with $V$ linearly (as expected by $[1,16]$ ) while the utility associated to the CLC2b algorithm is converging to the optimal point like $\frac{1}{V}$. The solution of CLC2b algorithm for different $V$ parameters are also shown in Figure 4.3 (shown by the various asterisks). We can observe that as $V$ gets larger the solution of CLC2b algorithm converges to the optimal point.
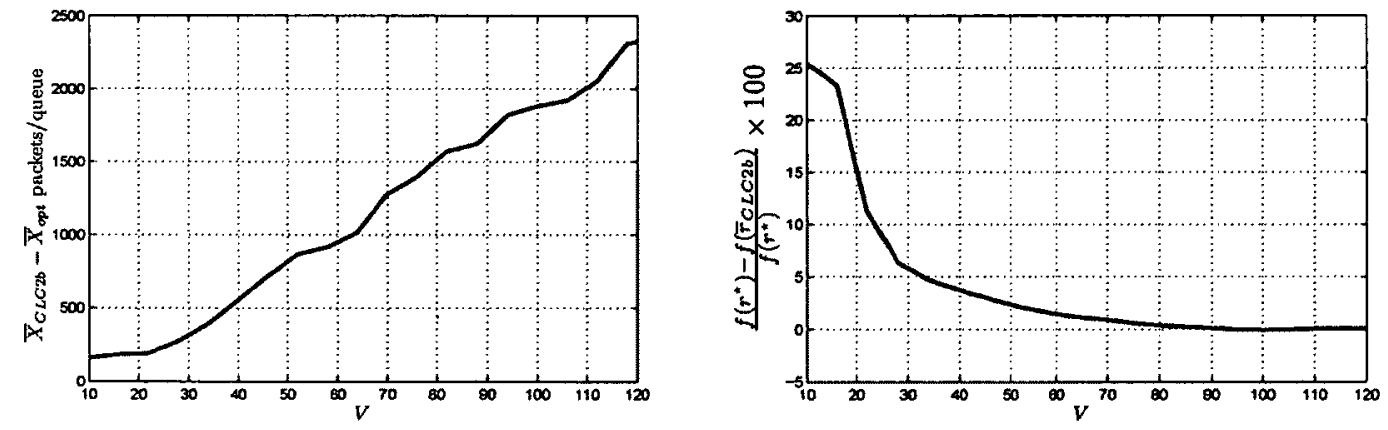

(a) Difference of the average queue lengths for (b) Percentage of the normalized difference of the optimal solution and the CLC2b algorithm the utility function for $\bar{r}$ and $r^{\star}$

Figure 4.5: Comparison of the performances of CLC2b algorithm with the optimal solution 


\subsubsection{Stability Region for Fluid Model MQMS Systems with Stationary Continuous Channel Distribution}

Consider a time-slotted fluid model MQMS system with stationary channel distribution. In this case, the amount of work that arrives into (and departs from) the queues is considered to be a continuous process. Also assume that the channel state of the link from each queue to each server is modeled by a continuous random variable. The channel state matrix in the fluid model MQMS system is defined as $C(t)=\left(C_{n, k}(t)\right), n \in \mathcal{N}, k \in \mathcal{K}, C_{n, k}(t) \in \mathbb{R}_{+}$. Assume that the channel process follows a stationary distribution $f_{C(t)}(c)$ where $C(t), c \in \mathbb{R}_{+}^{N \times K}$; i.e., $f_{C}(\cdot)$ is the joint distribution of all $C_{n, k}(t)$ variables. We can easily check that in the fluid model MQMS system, set $\widehat{V}$ is equivalent to $\mathbb{R}_{+}^{N}$ as in the fluid model MQMS system, set $\mathcal{M}$ is replaced with $\mathbb{R}_{+}$. Therefore, stability region for the fluid model system is characterized by the following set of inequalities.

$$
\begin{aligned}
\alpha \lambda^{\mathrm{T}} \leq \int_{c_{n, k}=0}^{\infty} \int_{c_{n, k-1}=0}^{\infty} \cdots \int_{c_{1,1}=0}^{\infty} \max _{I \in \mathcal{I}}\left(\alpha(c \circledast I) \underline{1}_{K}^{\mathrm{T}}\right) \\
\times f_{C(t)}(c) d c_{1,1} \cdots d c_{n, k-1} d c_{n, k} \quad \alpha \in \mathbb{R}_{+}^{N}
\end{aligned}
$$

Note that in order to characterize the stability region of the fluid model system we need to compute an infinite number of nested integrals in (4.32). In fact, the stability region of fluid model system is characterized by an infinite number of half spaces, hence the stability region is a convex surface. In this case, depending on the channel distribution and dimension of the system, we may characterize the stability region by a finite number of non-linear inequalities instead of infinite number of linear inequalities as it is shown in the following example.

Example: Consider a fluid model MQMS system with two queues and one server. Assume that the channel state variables $C_{1,1}(t)$ and $C_{2,1}(t)$ are independent and follow exponential distribution with means $\mu_{1}$ and $\mu_{2}$, respectively. Such a model may be used for slow Rayleigh fading channels in low SNR regimes where SNR follows exponential distribution and the approximation $\log (1+x) \simeq x$ is used for small positive $x$. According to (4.32), the stability region is characterized by

$$
\begin{array}{r}
\alpha_{1} \lambda_{1}+\alpha_{2} \lambda_{2} \leq \frac{1}{\mu_{1} \mu_{2}} \int_{c_{1,1}=0}^{\infty} \int_{c_{2,1}=0}^{\infty} \max \left\{\alpha_{1} c_{1,1}, \alpha_{2} c_{2,1}\right\} e^{-\frac{c_{1,1}}{\mu_{1}}} e^{-\frac{c_{2,1}}{\mu_{2}}} d c_{2,1} d c_{1,1} \\
\forall \alpha_{1}, \alpha_{2} \in \mathbb{R}_{+}
\end{array}
$$


We can write the right hand side of (4.33) as

$$
\begin{aligned}
& \frac{1}{\mu_{1} \mu_{2}} \int_{c_{1,1}=0}^{\infty} \int_{c_{2,1}=0}^{\frac{\alpha_{1}}{\alpha_{2}} c_{1,1}} \alpha_{1} c_{1,1} e^{-\frac{c_{1,1}}{\mu_{1}}} e^{-\frac{c_{2,1}}{\mu_{2}}} d c_{2,1} d c_{1,1} \\
& +\frac{1}{\mu_{1} \mu_{2}} \int_{c_{1,1}=0}^{\infty} \int_{c_{2,1}=\frac{\alpha_{1}}{\alpha_{2}} c_{1,1}}^{\infty} \alpha_{2} c_{2,1} e^{-\frac{c_{1,1}}{\mu_{1}}} e^{-\frac{c_{2,1}}{\mu_{2}}} d c_{2,1} d c_{1,1} \\
& =\frac{\alpha_{1}}{\mu_{1} \mu_{2}} \int_{c_{1,1}=0}^{\infty} c_{1,1} e^{-\frac{c_{1,1}}{\mu_{1}}} \int_{c_{2,1}=0}^{\frac{\alpha_{1}}{\alpha_{2}} c_{1,1}} e^{-\frac{c_{2,1}}{\mu_{2}}} d c_{2,1} d c_{1,1} \\
& +\frac{\alpha_{2}}{\mu_{1} \mu_{2}} \int_{c_{1,1}=0}^{\infty} e^{-\frac{c_{1,1}}{\mu_{1}}} \int_{c_{2,1}=\frac{\alpha_{1}}{\alpha_{2}} c_{1,1}}^{\infty} c_{2,1} e^{-\frac{c_{2,1}}{\mu_{2}}} d c_{2,1} d c_{1,1} \\
& =\alpha_{1}\left(\mu_{1}-\frac{\alpha_{2}^{2} \mu_{2}^{2} \mu_{1}}{\left(\alpha_{1} \mu_{1}+\alpha_{2} \mu_{2}\right)^{2}}\right)+\alpha_{2}\left(\frac{\alpha_{1} \alpha_{2} \mu_{2}^{2} \mu_{1}}{\left(\alpha_{1} \mu_{1}+\alpha_{2} \mu_{2}\right)^{2}}+\frac{\alpha_{2} \mu_{2}^{2}}{\alpha_{1} \mu_{1}+\alpha_{2} \mu_{2}}\right)
\end{aligned}
$$

Hence, all the ordered pairs $\left(\lambda_{1}, \lambda_{2}\right)=\left(\mu_{1}-\frac{\alpha_{2}^{2} \mu_{2}^{2} \mu_{1}}{\left(\alpha_{1} \mu_{1}+\alpha_{2} \mu_{2}\right)^{2}}, \frac{\alpha_{1} \alpha_{2} \mu_{2}^{2} \mu_{1}}{\left(\alpha_{1} \mu_{1}+\alpha_{2} \mu_{2}\right)^{2}}+\frac{\alpha_{2} \mu_{2}^{2}}{\alpha_{1} \mu_{1}+\alpha_{2} \mu_{2}}\right)$ characterize the boundary of the stability region. However, we can write $\lambda_{2}$ based on $\lambda_{1}, \mu_{1}$ and $\mu_{2}$ as follows.

$$
\mu_{1}-\frac{\alpha_{2}^{2} \mu_{2}^{2} \mu_{1}}{\left(\alpha_{1} \mu_{1}+\alpha_{2} \mu_{2}\right)^{2}}=\lambda_{1}
$$

So,

$$
\frac{\alpha_{2} \mu_{2}}{\alpha_{1} \mu_{1}+\alpha_{2} \mu_{2}}=\sqrt{1-\frac{\lambda_{1}}{\mu_{1}}} \quad \text { and } \quad \frac{\alpha_{1} \mu_{1}}{\alpha_{1} \mu_{1}+\alpha_{2} \mu_{2}}=1-\sqrt{1-\frac{\lambda_{1}}{\mu_{1}}}
$$

Therefore,

$$
\lambda_{2}=\mu_{2}\left(\sqrt{1-\frac{\lambda_{1}}{\mu_{1}}}\right)\left(2-\sqrt{1-\frac{\lambda_{1}}{\mu_{1}}}\right)
$$

As we can see from equation (4.35), the stability region in this example is characterized by just one non-linear inequality $\lambda_{2} \leq \mu_{2}\left(\sqrt{1-\frac{\lambda_{1}}{\mu_{1}}}\right)\left(2-\sqrt{1-\frac{\lambda_{1}}{\mu_{1}}}\right)$ and two linear inequalities $\lambda_{1} \geq 0$ and $\lambda_{2} \geq 0$. The stability region for this example for $\mu_{1}=2$ and $\mu_{2}=1$ is illustrated in Figure 4.6. 


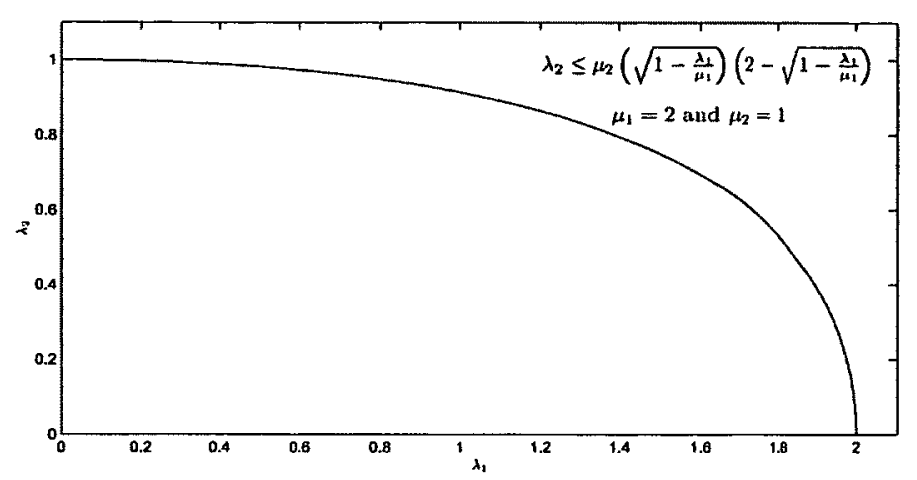

Figure 4.6: Stability region for $\mu_{1}=2$ and $\mu_{2}=1$

\subsection{Summary}

In this chapter, we introduced a linear algebraic representation of the network stability region (capacity region) polytope of multi-queue multi-server (MQMS) queueing system with stationary channel distribution and stationary arrival processes. To this end, we obtained the necessary and sufficient conditions for the stability of the system for a general arrival process with finite first and second moments. For stationary arrival processes, we showed that these conditions establish the network stability region of the system given by (4.18). For the stability region polytope, we explicitly determined all the coefficients $\alpha \in \widehat{V}$ of all the half spaces which are required to characterize the stability region polytope. We also argued that in general it may be (computationally) hard to quantify set $\widehat{V}$. In this case, although we may add some redundant inequalities, we can use a superset of $\widehat{V}$, namely the set $\mathcal{W}^{N}-\left\{\underline{0}_{N}\right\}$ $\left(\widehat{V} \subseteq \mathcal{W}^{N}-\left\{\underline{0}_{N}\right\}\right)$ instead of $\widehat{V}$ in (4.18). An upper bound was also obtained for the average queueing delay of Maximum Weight (MW) server allocation policy which is a throughput-optimal policy for MQMS system. We finally considered the stability region for a fluid model MQMS. In this case, we determine the stability region by an infinite set of linear inequalities given by (4.32). By use of an example we showed that depending on the channel distribution and the number of queues, we may characterize the stability region by a finite set of non-linear inequalities instead of infinite number of linear inequalities. However, the general problem of stability region characterization for the fluid model MQMS can be considered as a possible future research problem. 


\section{Chapter 5}

\section{Optimal Server Assignment in Symmetric Multi-server Single-interface Queueing Systems with Random Connectivities}

In this chapter, we will consider again the multi-server, single-interface queueing system with random connectivities introduced in Section 3.3 of Chapter 3. In Chapter 3 , we studied the stability properties of this system and we characterized the system stability region by a finite set of linear inequalities. In our analysis in Chapter 3 , we studied the system under the following general assumptions for the system connectivity and arrivals:

- The connectivity of the system (the connectivity matrix $C(t)$ ) is a stationary process represented by $\{C(t)\}_{t=1}^{\infty}$ with stationary probabilities $P(c), c \in \mathcal{C}$ where $\mathcal{C}$ is the set of all $N \times K$ binary matrices.

- The arrivals where assumed to follow any general distribution with finite first and second moments.

In this chapter, we will study a similar system with stronger assumptions on the connectivity and arrival processes. More specifically, we will consider the same system with the following assumptions on the connectivity and arrival processes:

- $C_{n, k}(t)$ 's are independent and $E\left[C_{n, k}(t)\right]=p$ for all $n \in \mathcal{N}, k \in \mathcal{K}$ and $t=$ $1,2, \ldots$

- The arrival random variables $A_{n}(t)$ 's are i.i.d. Bernoulli random variables with the same parameter $\lambda$ for all $n$ and $t$. 
We define a multi-server, single-interface queueing system with such assumptions on the connectivity and arrival processes as a symmetric system.

As we explained in Chapter 3, it has been previously proven that Maximum Weighted Matching (MWM) is a throughput-optimal server assignment policy for multi-server, single-interface queueing systems with random connectivities $[4,5]$. In this chapter, our contribution is to prove that for a symmetric system with i.i.d. Bernoulli packet arrivals and connectivities, MWM minimizes, in stochastic ordering sense $^{1}$, a broad range of cost functions of the queue lengths including total queue occupancy (or equivalently minimizes the average queueing delay). Then, we extend the model by considering imperfect services where it is assumed that the service of a scheduled packet fails randomly with a certain probability. We prove that the same policy is still optimal for the extended model. We also show that the results are still valid for some extended connectivity and arrival processes (e.g., Poisson arrival process). For the sake of consistency and ease of reading, we will briefly describe the system in the following section.

\subsection{System Description}

We will consider a multi-server, single-interface queueing system with random connectivities between the queues and the servers. The system consists of a set of identical servers namely $\mathcal{K}=\{1,2, \ldots, K\}$ and a set of parallel queues $\mathcal{N}=\{1,2, \ldots, N\}$ with infinite buffer space for each queue (See Figure 5.1). Packets in this system are assumed to have constant length and require one time slot to complete service. The connectivity of each queue $n \in \mathcal{N}$ to each server $k \in \mathcal{K}$ at each time slot $t$ is random and varying across time slots. We denote the connectivity of queue $n$ to server $k$ at time slot $t$ by $C_{n, k}(t) \in\{0,1\}$. At each time slot $t$, the connectivity state may be expressed by an $N \times K$ matrix $C(t)=\left(C_{n, k}(t), n \in \mathcal{N}, k \in \mathcal{K}, C_{n, k}(t) \in\{0,1\}\right)$. We assume that $C_{n, k}(t)$ 's are independent and $E\left[C_{n, k}(t)\right]=p$ for all $n \in \mathcal{N}, k \in \mathcal{K}$ and $t=1,2, \ldots$. In other words, all the connectivity variables in the system are assumed to be independent and identically distributed according to a Bernoulli distribution with parameter $p$.

\footnotetext{
${ }^{1}$ We order two discrete time random processes $A=\{A(t)\}_{t=1}^{\infty}$ and $B=\{B(t)\}_{t=1}^{\infty}$ stochastically as follows: We say $A$ is stochastically less than $B$ and we write $A \leq_{\text {st }} B$ if $\operatorname{Pr}(A(t)>r) \leq \operatorname{Pr}(B(t)>r)$ for all $t=1,2, \ldots$ and all $r \in \mathbb{R}$. The notion and relevant properties will be discussed in more detail in Section 5.2.1.
} 


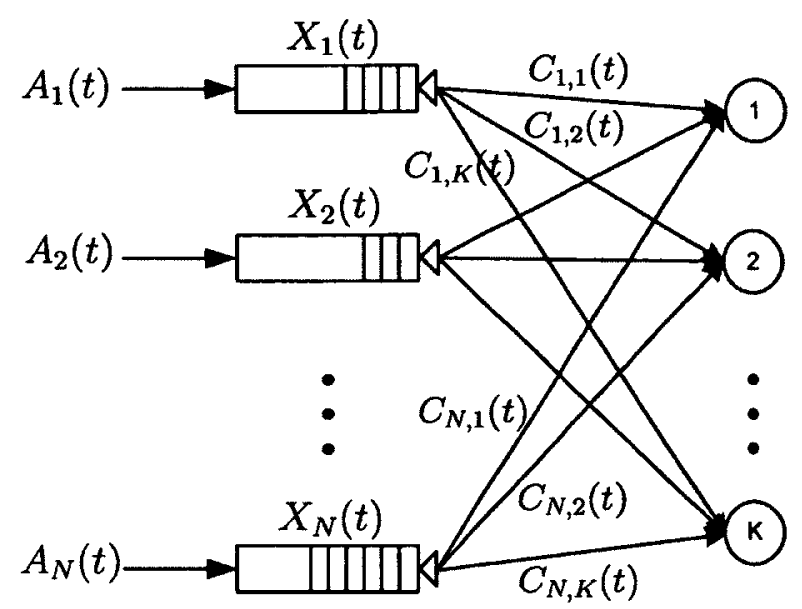

Figure 5.1: Discrete time queueing system with $N$ parallel queues and $K$ servers

At any time slot, each server can serve at most one packet from a connected nonempty queue. Note that in the system we do not allow server sharing, i.e., a server can serve at most one queue per time slot. Since the queues are single-interface queues, they can get service from at most one server in each time slot.

Let $A_{n}(t)$ be the packet arrival random variable (number of packet arrivals) to queue $n$ at time slot $t$. We assume that new arrivals at each time slot are added to the queues at the end of time slots. The arrival variables $A_{n}(t)$ 's are assumed to be i.i.d. Bernoulli random variables with a given parameter $\lambda$ for all $n$ and $t$. We denote the length of queue $n$ at the end of time slot $t$ (i.e., after adding the new arrivals) by $X_{n}(t)$. In other words, $X_{n}(t)$ represents the number of packets in the $n$th queue at the end of time slot $t$ (or beginning of time slot $t+1$ ).

As we explained in Section 3.3.1, a server assignment policy at each time slot determines a bipartite matching (matching in bipartite graphs) between sets $\mathcal{N}$ and $\mathcal{K}$. This should be accomplished based on the available information about the connectivities $C_{n, k}(t)$ and also the queue length vector process at the beginning of time slot $t$ (which is $X(t-1)=\left(X_{1}(t-1), X_{2}(t-1), \ldots, X_{N}(t-1)\right)$ ). Using similar notation as in Chapter 3, we define the $N \times K$ matrix $I^{(\pi)}(t)=\left(I_{n, k}^{(\pi)}(t), \forall n \in \mathcal{N}, k \in \mathcal{K}\right)$ as the employed matching by policy $\pi$ at time slot $t$ where indicator variables $I_{n, k}^{(\pi)}(t)$ 's are defined as in Section 3.3.1, i.e., $I_{n, k}^{(\pi)}(t)$ is " 1 " if server $k$ is assigned to queue $n$ at time slot $t$ and " 0 " otherwise. Therefore, a server scheduling policy $\pi$ is defined as $\pi=\left\{I^{(\pi)}(t)\right\}_{t=1}^{\infty}$. The matching space is defined similar to what was defined in Section 3.3.1, i.e., 
$\mathcal{I}=\left\{I_{N \times K}=\left(I_{n, k}, \forall n \in \mathcal{N}, \forall k \in \mathcal{K}\right) \mid \sum_{k=1}^{K} I_{n, k} \leq 1 \quad \forall n \in \mathcal{N}, \sum_{n=1}^{N} I_{n, k} \leq 1 \quad \forall k \in \mathcal{K}\right\}$

According to the above discussion, we can see that the queue length random process $\{X(t)\}_{t=0}^{\infty}$ evolves with time according to the following rule.

$$
X_{n}(t)=\left(X_{n}(t-1)-\sum_{k=1}^{K} C_{n, k}(t) I_{n, k}^{(\pi)}(t)\right)^{+}+A_{n}(t) \quad \forall n \in \mathcal{N}
$$

in which $C_{n, k}(t) \in\{0,1\}, I_{n, k}^{(\pi)}(t) \in\{0,1\}, A_{n}(t) \in\{0,1\}$ for all $n \in \mathcal{N}$ and $k \in \mathcal{K}$.

Note that the system introduced above is different from the system studied in $[63,65,89]$ from the following aspects:

- In the system studied in $[63,65,89]$, there is no restriction on the number of servers that can be allocated to each server. However, in the model presented above, we restrict each queue to attract at most one server per time slot. Even adding such a small extra assumption makes the analysis of the system different from the analysis used in $[63,65,89]$.

- In $[63,89]$, the authors proved the optimality of a class of Most Balancing policies (which are parametrized by minimization of a specific cost function) without any implementation for the optimal policy. However, for the studied system in this chapter, we parametrize the optimal policy by maximization of a reward function which also determines the construction of the optimal policy (i.e., MWM policy). Therefore, for this system we also determine the implementation of the optimal policy.

- For the model studied in $[63,65,89]$, we showed in [14] that the back-pressure algorithm is not delay-optimal even for a symmetric case. However, as we will see in the rest of the chapter, we prove that for the system studied in this chapter the back-pressure algorithm (or MWM policy) is delay optimal in a symmetric system. This demonstrates the fact that the back-pressure algorithm is not delay-optimal for all symmetric queueing systems. 


\subsection{Optimality of MWM Policy}

We first review the concepts of stochastic ordering and dynamic coupling arguments in the following subsection. Then, we will prove that the optimality of MWM policy is beyond the maximization of the stable throughput region. Indeed, we will prove that in a symmetric, multi-server, single-interface queueing system, MWM policy is optimal in minimizing, in stochastic ordering sense, a class of objective functions of queue length process. Such a class of objective functions includes the total queue occupancy and therefore queueing delay. For brevity, we will use the term "delay optimality" to refer to the optimality of MWM in this sense. Note that optimality in stochastic ordering sense will result in optimality in mean. Thus, a delay-optimal policy has the minimum average queueing delay among all the possible server assignment policies.

\subsubsection{Stochastic Ordering and Dynamic Coupling}

In this section, we briefly review the concepts of stochastic ordering (stochastic dominance) and dynamic coupling techniques. Consider two discrete-time stochastic processes $A=\{A(t)\}_{t=1}^{\infty}$ and $B=\{B(t)\}_{t=1}^{\infty}$ in $\mathbb{R}$. We say $A$ is stochastically smaller than $B$ and we write $A \leq_{s t} B$ if $\operatorname{Pr}(A(t)>r) \leq \operatorname{Pr}(B(t)>r)$ for all $t=1,2, \ldots$ and all $r \in \mathbb{R}[90,91]$. Some properties of stochastic ordering are the following. If $A \leq_{s t} B$ then $E[A(t)] \leq E[B(t)]$. If $A \leq_{s t} B$ then $f(A) \leq_{s t} f(B)$ for all non-decreasing functions f. $A$ is stochastically smaller than $B$, if there exists process $\tilde{A}=\{\tilde{A}(t)\}_{t=1}^{\infty}$ defined on the same probability space as $B$ with the same probability distribution as $A$ and satisfies $\tilde{A}(t) \leq B(t)$ almost surely for every $t=1,2, \ldots[62]$. The last statement is known as coupling of $A$ and $\tilde{A}$. In fact, when applying coupling technique, given process $A$ we try to construct a coupled process $\tilde{A}$ with the same distribution

as $A$ and $\tilde{A}(t) \leq B(t)$ a.s. for all $t$. This property provides us a tool for comparing the processes $A$ and $B$ stochastically when it is infeasible to derive the distributions of $A$ and $B$ (e.g., in our queueing model when comparing the total occupancy process for different server assignment policies).

\subsubsection{Delay Optimality of MWM}

In this section, we present the main result of this chapter, that is proving the optimality of MWM with respect to minimization of a class of cost functions of queue 
lengths including the average queueing delay for the symmetric multi-server queueing system described in Section 5.1. Suppose that $\mathbb{Z}_{+}$is the set of non-negative integers and $\mathbb{Z}_{+}^{N}$ is the $N$ dimensional Cartesian space of non-negative integers. We define relation " $\preceq$ " on $\mathbb{Z}_{+}^{N}$ as follows.

Definition 5.1 For two vectors $x, \tilde{x} \in \mathbb{Z}_{+}^{N}$, we write $\tilde{x} \preceq x$ if one of the following relations holds:

D1: $\tilde{x}_{n} \leq x_{n}$ for all $n=1,2, \ldots, N$.

D2: $\tilde{x}$ is obtained by permutation of two distinct elements of $x$, i.e., $\tilde{x}$ and $x$ are different in only two elements $n$ and $m$ such that $\tilde{x}_{n}=x_{m}$ and $\tilde{x}_{m}=x_{n}$.

D3: $\tilde{x}$ and $x$ are different in only two elements $n$ and $m$ such that $x_{n}<\tilde{x}_{n} \leq \tilde{x}_{m}<$ $x_{m}$ and the following constraints are satisfied: $\tilde{x}_{n}=x_{n}+1$ and $\tilde{x}_{m}=x_{m}-1$.

In D3, we say that $\tilde{x}$ is more balanced than $x$ and can be obtained by decreasing a larger element of $x$ (i.e., $m$ ) by " 1 " and increasing a smaller element (i.e., $n$ ) by "1". We call such an interchange as a balancing interchange on vector $x$. Thus, the result of a balancing interchange on a vector $x$ would be a vector $\tilde{x}$ such that $\tilde{x} \preceq x$. Suppose that vector $x \in \mathbb{Z}_{+}^{N}$ represents the queue length vector at a given time slot. Then a balancing interchange is equivalent to taking a packet from a larger queue and adding it to a smaller queue.

We define the partial order " $\preceq_{p}$ " on $\mathbb{Z}_{+}^{N}$ as the transitive closure of relation

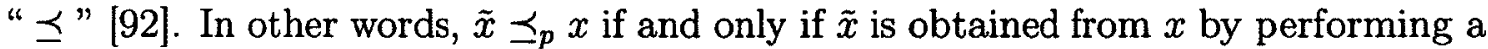
sequence of reductions, permutations of two elements and/or balancing interchanges. When $x$ and $\tilde{x}$ are two queue length vectors, we write $\tilde{x} \preceq_{p} x$ if and only if queue length vector $\tilde{x}$ is obtained from $x$ by applying a series of packet removals, two-queue permutations and balancing interchanges.

We define $\mathcal{F}$ as the class of real-valued functions on $\mathbb{Z}_{+}^{N}$ that are monotone and non-decreasing with respect to the partial order $\preceq_{p}$, i.e.,

$$
f \in \mathcal{F} \Longleftrightarrow \tilde{x} \preceq_{p} x \Rightarrow f(\tilde{x}) \leq f(x)
$$

We can easily check that function $f(x)=\sum_{n=1}^{N} x_{n}$ belongs to $\mathcal{F}$. This function represents the total queue occupancy of the system. 


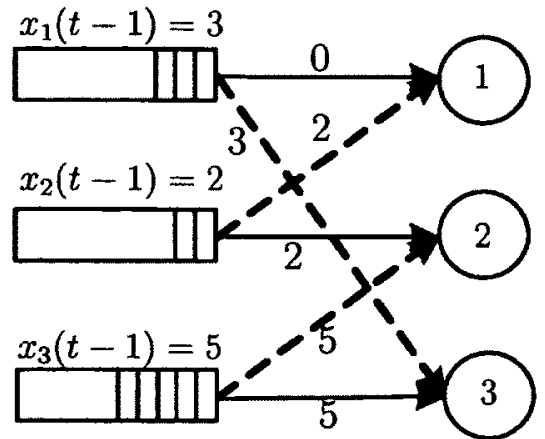

(a) Satisfying condition C1

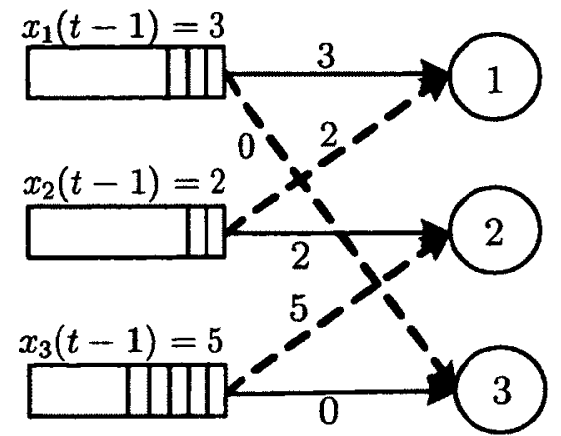

(b) Satisfying condition C2

Figure 5.2: Examples of balancing server reallocations (the weight $c_{n, k}(t) x_{n}(t-1)$ of each edge $(n, k)$ is also shown)

Let $X^{\prime}(t)=\left(X_{1}^{\prime}(t), X_{2}^{\prime}(t), \ldots, X_{N}^{\prime}(t)\right)$ denote the queue length vector at time slot $t$ exactly after serving the queues according to a server assignment policy $\pi$ and before adding the new arrivals of time slot $t$, i.e.,

$$
X_{n}^{\prime}(t)=\left(X_{n}(t-1)-\sum_{k=1}^{K} C_{n, k}(t) I_{n, k}^{(\pi)}(t)\right)^{+}
$$

Given $x^{\prime}(t)$ as a sample value of random vector $X^{\prime}(t)$, we define a balancing server reallocation at time slot $t$ as follows.

Definition $5.2 A$ balancing server reallocation ${ }^{2}$ on vector $x^{\prime}(t)$ is a matching that results in vector $\tilde{x}^{\prime}(t)$ such that one of the following conditions is satisfied.

(C1) $\tilde{x}_{n}^{\prime}(t) \leq x_{n}^{\prime}(t)$ for all $n=1,2, \ldots, N$ and there exists $m \in\{1,2, \ldots, N\}$ such that $\tilde{x}_{m}^{\prime}(t)<x_{m}^{\prime}(t)$.

(C2) $\tilde{x}^{\prime}(t)$ and $x^{\prime}(t)$ are different in only two elements $n$ and $m$ such that $x_{n}^{\prime}(t)<$ $\tilde{x}_{n}^{\prime}(t) \leq \tilde{x}_{m}^{\prime}(t)<x_{m}^{\prime}(t)$ and the following constraints are satisfied: $\tilde{x}_{n}^{\prime}(t)=x_{n}^{\prime}(t)+1$ and $\tilde{x}_{m}^{\prime}(t)=x_{m}^{\prime}(t)-1$.

Figures 5.2(a) and 5.2(b) show two examples of balancing server reallocations in two sample graphs. In these figures, the original allocations are specified by solid lines while the balancing reallocations are specified by dashed lines.

\footnotetext{
${ }^{2} \mathrm{~A}$ balancing server reallocation may consist of one or more server reassignment.
} 
Consider an arbitrary server assignment policy $\pi$ with the allocation variables $\left\{I_{n, k}^{(\pi)}(t)\right\}_{t=1}^{\infty}$ for all $k \in \mathcal{K}$ and $n \in \mathcal{N}$. We introduce Matching Weight (MW) index associated to the server allocation policy $\pi$ at time slot $t$ by

$$
\mathrm{MW}_{\pi}(t)=\sum_{n=1}^{N} x_{n}(t-1) \sum_{k=1}^{K} c_{n, k}(t) I_{n, k}^{(\pi)}(t)
$$

Note that MW index is exactly the objective of the optimization problem (3.34) (See Section 3.3.2 in Chapter 3). According to Definition 5.2 and definition of MW index, we can prove the following Lemma.

Lemma 5.1 For a given policy $\pi$ employing matching $I^{(\pi)}(t)$ at time slot $t$, by applying a balancing server reallocation at time slot $t$ (if there exists any), we will have a new policy $\tilde{\pi}$ differing from $\pi$ only at time slot $t$ such that $\mathrm{MW}_{\pi}(t)<\mathrm{MW}_{\tilde{\pi}}(t)$.

The detailed proof of the lemma is given in Appendix D.1. Based on Lemma 5.1, we can state the following corollary.

Corollary 5.1 For a given policy $\pi$ at time slot $t$, if $\mathrm{MW}_{\pi}(t)$ is maximized, i.e., policy $\pi$ employs a maximum weighted matching at time slot $t$, then there exists no balancing server reallocation at that time slot.

Note that Lemma 5.1 states that any balancing reallocation increases the matching weight index. However, it does not imply the existence of a balancing server reallocation when $\mathrm{MW}_{\pi}(t)$ is not maximized. In the following, we will prove the existence result i.e., the reverse of Lemma 5.1.

Lemma 5.2 For a given policy $\pi$ at time slot $t$, if $\mathrm{MW}_{\pi}(t)$ is not maximized, i.e., $\mathrm{MW}_{\pi}(t)<\mathrm{MW}_{M W M}(t)$, then there exists a balancing server reallocation at that time slot.

For the detailed proof, please refer to Appendix D.2.

By $\Pi^{\mathrm{MWM}}$, we denote the set of all policies which employ maximum weighted matching at all time slots. We also define $\Pi_{t}$ as the set of all policies that employ maximum weighted matching exactly until time slot $t$ (including $t$ ). We can easily observe that $\Pi_{t-1} \supseteq \Pi_{t}$ and $\Pi^{\mathrm{MWM}}=\bigcap_{t=1}^{\infty} \Pi_{t}$. From Lemmas 5.1 and 5.2, we conclude that given a policy $\pi \in \Pi_{t-1}$ which is using an arbitrary matching at time slot $t$, we can reach a policy $\pi^{\star} \in \Pi_{t}$ by applying a sequence of balancing server reallocations. 
Suppose that $h_{t}^{\pi}$ represents the number of balancing server reallocations required to convert the employed matching in policy $\pi$ at time slot $t$ to a maximum weighted matching. In this case, we say that the distance of $\pi$ from $\Pi_{t}$ is $h_{t}^{\pi}$ balancing server reallocations. Note that if the distance of $\pi$ from $\Pi_{t}$ is $h_{t}^{\pi}$, after applying the first balancing server reallocation we get to a policy $\tilde{\pi}$ whose distance from $\Pi_{t}$ is $h_{t}^{\pi}-1$ balancing server reallocations. By repeating this procedure we finally get to a policy whose distance to $\Pi_{t}$ is zero, i.e., it belongs to $\Pi_{t}$. By $\Pi_{t}^{h}\left(0 \leq h \leq h_{t}^{\pi}\right)$ we denote the set of all server assignment policies in $\Pi_{t-1}$ whose distance from $\Pi_{t}$ is at most $h$ balancing sever reallocations. Note that $\Pi_{t}^{0}=\Pi_{t}$.

Consider any two policies $\pi$ and $\tilde{\pi}$ such that $f(\tilde{X}) \leq_{s t} f(X)$ where $X=\{X(t)\}_{t=1}^{\infty}$ and $\tilde{X}=\{\tilde{X}(t)\}_{t=1}^{\infty}$ are the queue length processes when policies $\pi$ and $\tilde{\pi}$ are applied, respectively. For such a system, we say policy $\tilde{\pi}$ dominates $\pi$. If $\tilde{\pi}$ dominates $\pi$ we have $E[f(\tilde{X})] \leq E[f(X)]^{3}$. Based on the above discussion about stochastic dominance, we can prove the following lemma.

Lemma 5.3 For any policy $\pi \in \Pi_{t}^{h}$ and $0<h \leq h_{t}^{\pi}$ we can construct a policy $\tilde{\pi} \in \Pi_{t}^{h-1}$ such that $\tilde{\pi}$ dominates $\pi$.

For the detailed proof, please refer to Appendix D.3. Using Lemma 5.3, we can prove the main result of this section in the following theorem.

Theorem 5.1 The Maximum Weighted Matching policy dominates any server assignment policy.

Proof: Let $\pi_{0}$ be any arbitrary policy. Then $\pi_{0} \in \Pi_{0}=\Pi_{1}^{H_{1}}$ where $H_{1}=h_{1}^{\pi_{0}}$. By applying Lemma 5.3 repeatedly, we can construct a sequence of policies such that each policy dominates the previous one. Thus, we obtain policies that belong to $\Pi_{0}=\Pi_{1}^{H_{1}}, \Pi_{1}^{H_{1}-1}, \Pi_{1}^{H_{1}-2}, \ldots, \Pi_{1}^{0}=\Pi_{1}$. The last policy is called $\pi_{1}$. Note that $\pi_{1} \in \Pi_{2}^{H_{2}}$ where $H_{2}=h_{2}^{\pi_{1}}$. By recursively continuing such an argument we obtain a sequence of policies $\pi_{t} \in \Pi_{t}, t=1,2, \ldots$ such that $\pi_{j}$ dominates $\pi_{i}$ for $j>i$. Note that this sequence of policies defines a limiting policy $\pi^{*}$ that agrees with MWM at all time slots. Thus, $\pi^{*}$ is an MWM policy which dominates all the previous policies, including the starting policy $\pi_{0}$.

\footnotetext{
${ }^{3}$ Choosing $f(x)=\sum_{n=1}^{N} x_{n}$, we conclude that the average queue occupancy (or equivalently average queueing delay) of policy $\tilde{\pi}$ is smaller than that of policy $\pi$.
} 


\subsubsection{Extensions}

\section{Imperfect Services}

We can extend Theorem 5.1 for the case where the service of a scheduled packet by a connected server fails randomly with a certain probability. This is analogous to realistic wireless networks operation where service failures usually occur due to unexpected and unpredictable effects of noise, interference, etc. In the case of a packet service failure, the packet will be kept in the queue and will be rescheduled and retransmitted in future time slots.

We denote the binary random variable associated to successful/unsuccessful service of queue $n$ provided by server $k$ at time slot $t$ by $Q_{n, k}(t)$. Note that $Q_{n, k}(t) \in$ $\{0,1\}$ and is " 1 " (" 0 ") when the service is successful (unsuccessful). We assume that $Q_{n, k}(t)$ 's are i.i.d. Bernoulli random variables with the same success probability $q$. Note that parameter $q$ (the same as parameters $\lambda$ and $p$ ) does not appear in our analysis and in fact what is necessary in our analysis is the symmetry of the system (i.e., $E\left[Q_{n, k}(t)\right]=q, \forall n, k$ ). The queue lengths are then updated at the end of each time slot by the following rule.

$$
X_{n}(t)=\left(X_{n}(t-1)-\sum_{k=1}^{K} C_{n, k}(t) I_{n, k}^{(\pi)}(t) Q_{n, k}(t)\right)^{+}+A_{n}(t) \quad \forall n \in \mathcal{N}
$$

Note that the network controller cannot observe the variables $Q_{n, k}(t)$ and from his perspective they are assumed to be random. The random vector $X^{\prime}(t)$ is defined similar to equation (5.3). Hence, $X^{\prime}(t)$ represents the queue lengths before adding the new arrivals of time slot $t$ as if all the services at that time slot are successful.

For such a system we can easily see that Lemmas 5.1 and 5.2 are still valid. We can also extend Lemma 5.3 for the system with service failures by considering random variables $Q_{n, k}(t)$ in our dynamic coupling argument. The detailed analysis is brought in Appendix D.4. By applying the same approach as what we did for Theorem 5.1, we can similarly prove the delay optimality of MWM policy for the system with imperfect services. 


\section{Extensions for Connectivity and Arrival Processes}

The arguments in Lemma 5.3 and Theorem 5.1 remain valid if the i.i.d. assumption for connectivity and arrival processes is relaxed as follows; we will consider connectivity and arrival processes which follow conditional permutation invariant distributions. Given event $\mathcal{H}$ (which is usually used to denote the history of the system), we define a conditional multivariate probability distribution $f\left(y_{1}, y_{2}, \ldots, y_{n} \mid \mathcal{H}\right)$ to be permutation invariant if for any permutation of the variables $y_{1}, y_{2}, \ldots, y_{n}$ namely $y_{1}^{\prime}, y_{2}^{\prime}, \ldots, y_{n}^{\prime}$ we have $f\left(y_{1}, y_{2}, \ldots, y_{n} \mid \mathcal{H}\right)=f\left(y_{1}^{\prime}, y_{2}^{\prime}, \ldots, y_{n}^{\prime} \mid \mathcal{H}\right)$. We can readily see that for all the connectivity and arrival processes whose joint distributions at each time slot given the history of the system ${ }^{4}$ (i.e., $f_{A(t)}\left(a_{1}, a_{2}, \ldots, a_{N} \mid \mathcal{H}\right.$ ) and $\left.f_{C(t)}\left(c_{1,1}, c_{1,2}, \ldots, c_{N, K-1}, c_{N, K} \mid \mathcal{H}\right)\right)$ are permutation invariant, Lemma 5.3 and Theorem 5.1 are still valid and therefore MWM is delay-optimal.

We also consider the generalization of Theorem 5.1 for non-Bernoulli arrival processes. Suppose that the number of arrivals to each queue can be represented by the summation of some i.i.d. Bernoulli random variables, i.e., has Binomial distribution. Also suppose that $A_{n}(t) \leq A_{\max }$ for all $n \in \mathcal{N}$ and all $t$. In this case, we can create a new (virtual) system in which after each time slot we append $A_{\max }-1$ virtual time slots and put the connectivities all equal to zero, i.e., for each virtual time slot $t, C_{n, k}(t)=0, \forall n \in \mathcal{N}, \forall k \in \mathcal{K}$. We then distribute the arrivals of the actual time slot among these $A_{\max }$ time slots (one actual time slot and $A_{\max }-1$ virtual time slots) randomly such that at each time slot at most one packet arrival occurs. Since the connectivities and the arrivals in both systems are permutation invariant, we can still prove Theorem 5.1 for the virtual system. Note that the operation of the two systems (the original system and the virtual system) are the same. Therefore, we can conclude that Theorem 5.1 is also valid for a multi-server system with Binomial arrival processes. As Poisson distribution asymptomatically approximates the Binomial distribution when $A_{\max }$ goes to infinity, Theorem 5.1 will be still valid for i.i.d. Poisson arrival processes.

\subsection{Simulation Results}

We have evaluated the performance of MWM policy with two other server assignment policies in terms of average queueing delay. The other two policies are described in

\footnotetext{
${ }^{4} \mathrm{By}$ history of the system we mean all the channel states, arrivals and matchings of the previous time slots.
} 
the following.

- Maximum Matching (MM) policy that applies the maximum matching on matrix $C(t)$. In other words, the maximum matching policy at each time slot $t$ employs a server assignment (or matching) $I^{(\mathrm{MM})}(t)$ which is obtained by $I^{(\mathrm{MM})}(t)=\arg \max _{M \in \mathcal{M}}(c(t) \circledast M) \underline{1}_{K}^{\mathrm{T}}$.

- A heuristic policy that assigns the servers to the queues at each time slot according to the following rule: It selects a server randomly and assigns it to its longest connected queue. Then, updates the set of servers by removing the selected server from $\mathcal{K}$ and the set of queues (i.e., $\mathcal{N}$ ) by removing the queue to which the selected server was assigned. This procedure is repeated $K$ times. Note that, for some servers the updated set $\mathcal{N}$ may be empty (e.g., when $K>N$ ) and therefore those servers are not assigned to any queue.

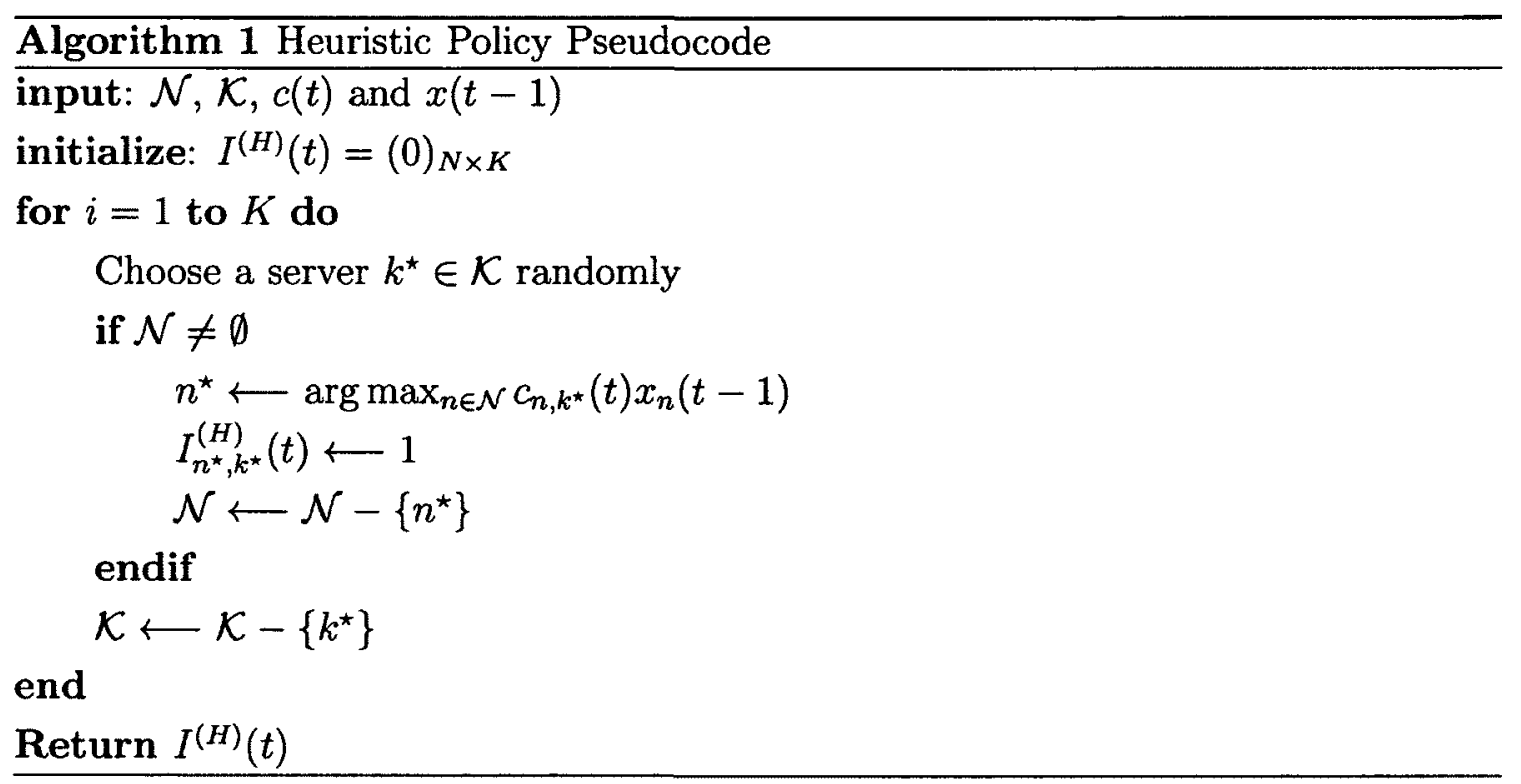

The main idea of the heuristic policy is coming from Longest Connected Queue (LCQ) policy which was proven to be optimal for a single server system [3]. For multi-server system, we will use the same principle for each server. However, the order in which the servers are selected for assignment is random. We have performed a comprehensive set of simulations in which we investigate the effects of number of servers $K$, probability of connectivity $p$ and probability of service success $q$ on the 
performance of the aforementioned policies. In all the simulations, we set $N=8$ and the arrivals are i.i.d. Poisson distributed.

Figures 5.3-5.5 illustrate the simulation results. As we can see in all the cases, MWM has better performance with respect to other policies in terms of average queue occupancy or average queueing delay. Figure 5.3 shows the simulation results for $K=4, q=0.8$ and $p=0.2,0.5,0.8$. In these cases, since the number of servers is relatively low, server assignment will be more competitive. MWM which was proven to be the optimal policy performs such that it tries to balance the queues. The heuristic policy follows the same principle. However, since the selection of servers for assignment is random, there are some cases where two or more servers have the same longest connected queue. In such cases, the order of selecting the servers for assignment is crucial in the performance of the system. Maximum Matching policy however, does not try to balance the queues. Indeed, it does not consider the queue lengths in its assignments and that is why it performs worse than the other two policies. Note that as the connectivity of the system gets larger, the performances of MWM and the heuristic policy get closer. It is worth mentioning that the heuristic policy introduced here performs the same as MWM for $K=1$ (which is equivalent to LCQ whose optimality was previously shown in [3]).

Figure 5.4 shows the results for 8 servers. In this case, since the number of servers is relatively high and comparable to the number of queues, in MWM and MM policies each queue gets service with high probability when the probability of server connectivities tends to 1 . As the connectivity probability gets smaller, the difference in performance of MWM and MM becomes more apparent. Note that in this case, the heuristic policy performs worse than the other two policies since it is more probable to lead to cases where two or more servers are connected to the same longest connected queue. As the number of severs becomes larger, we expect MM to perform the same as MWM as in this case the probability of serving all the queues increases. Therefore, in the limiting case where $K$ becomes very large, MM and MWM result in similar performance.

In Figure 5.5 we have investigated the effect of service success probability. As we can see in the figures, the only effect of this parameter is to change the stability point (the arrival rate at which queue occupancy tends to infinity). In this case, again we can see that for all $q=0.2,0.5,0.8 \mathrm{MWM}$ policy performs better than the other policies. 
Note that the delay optimality of MWM and LCQ policies were just proven for symmetric systems (with identical arrival rates and connectivity probability for all the queues). For an asymmetric system, we conjecture that a weighted version of MWM or LCQ may be optimal where the weights are dependent on the arrival rates as well as the connectivity probabilities.

\subsection{Summary}

In this chapter, we considered the problem of assignment of $K$ identical servers to a set of $N$ parallel queues in a time-slotted, multi-server, single-interface queueing system with random connectivities. For such systems, it has been previously shown that MWM is throughput optimal, i.e., has the maximum stability region. In this chapter, we showed that for a symmetric multi-server single-interface queueing system (with i.i.d. Bernoulli arrival and connectivity processes) MWM is also optimal in minimization of a class of cost functions of queue lengths including the average queueing delay. Our approach in the proof was based on stochastic ordering and dynamic coupling techniques. We also extended the results for more general arrival and connectivity processes and also for a system with imperfect service processes. Finally to validate our theoretical results, we conducted simulations to compare the performance of the MWM policy with that of two other policies in terms of average queue occupancy. 


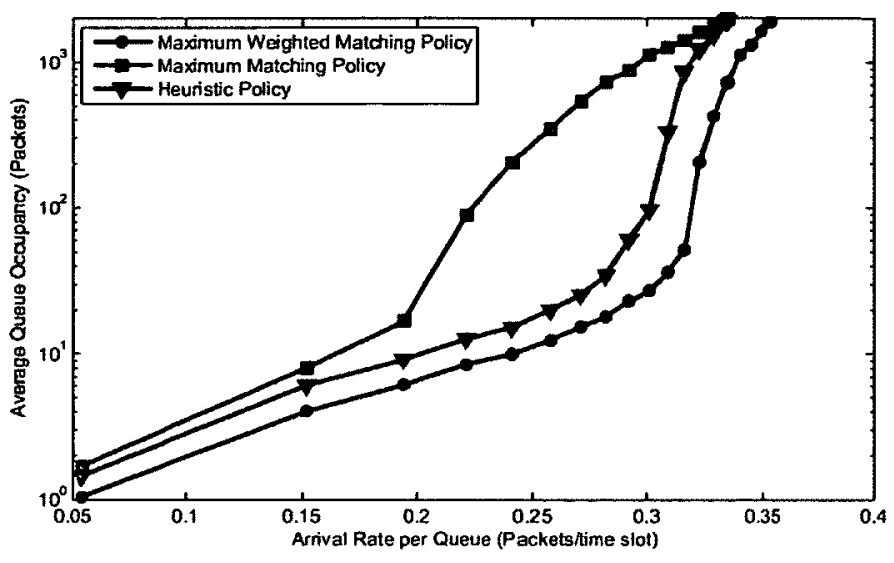

(a) $p=0.2$

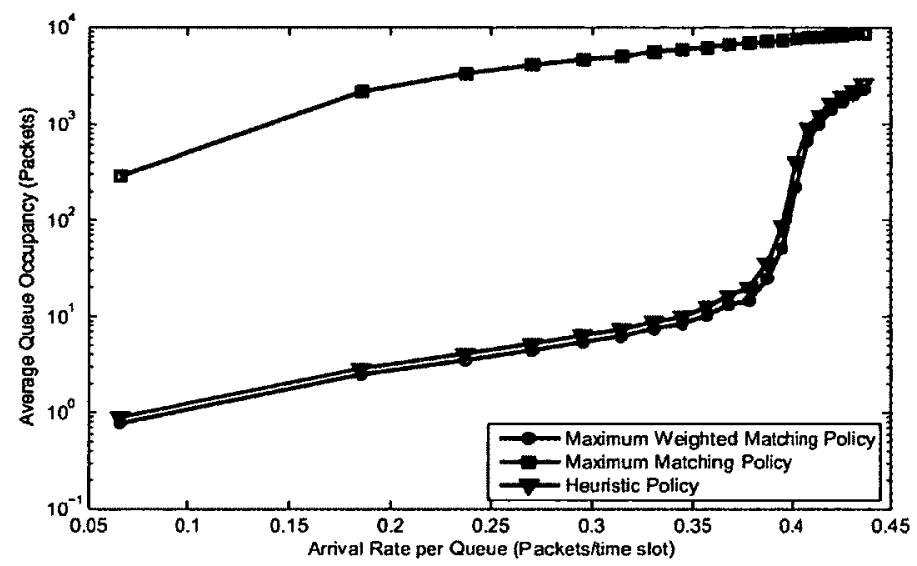

(b) $p=0.5$

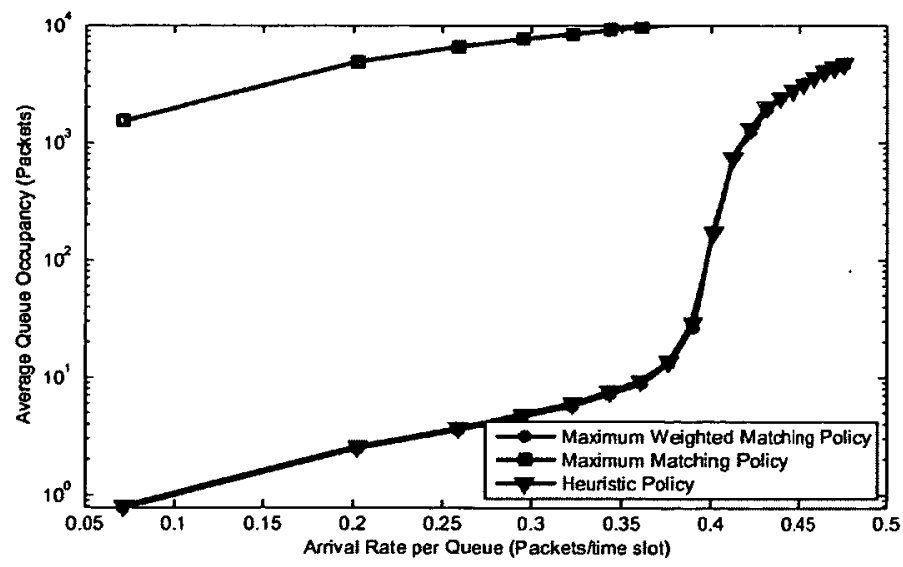

(c) $p=0.8$

Figure 5.3: Average total queue occupancy, $N=8, K=4, q=0.8$ 


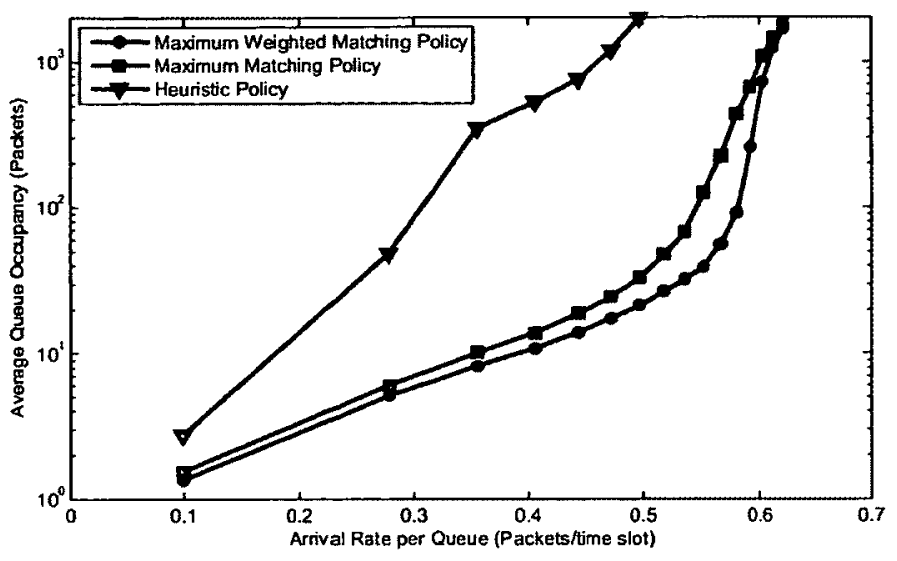

(a) $p=0.2$

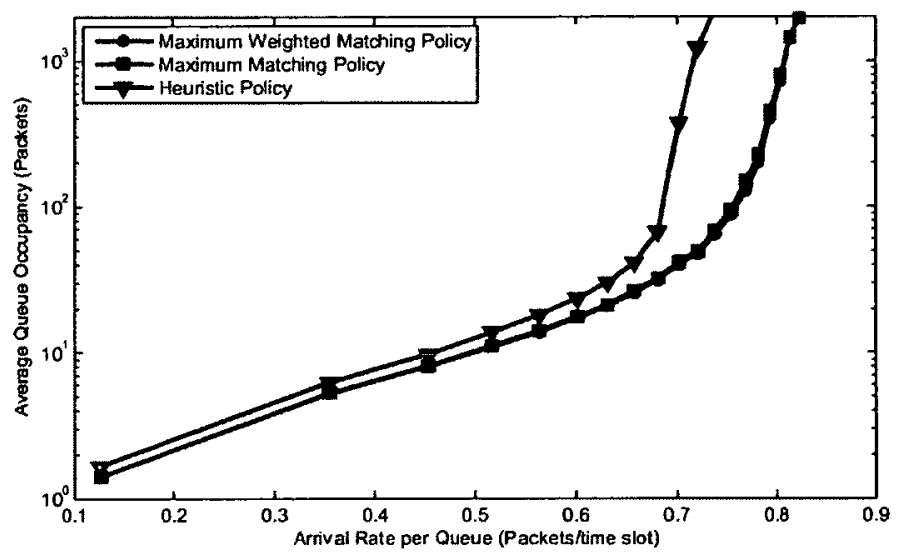

(b) $p=0.5$

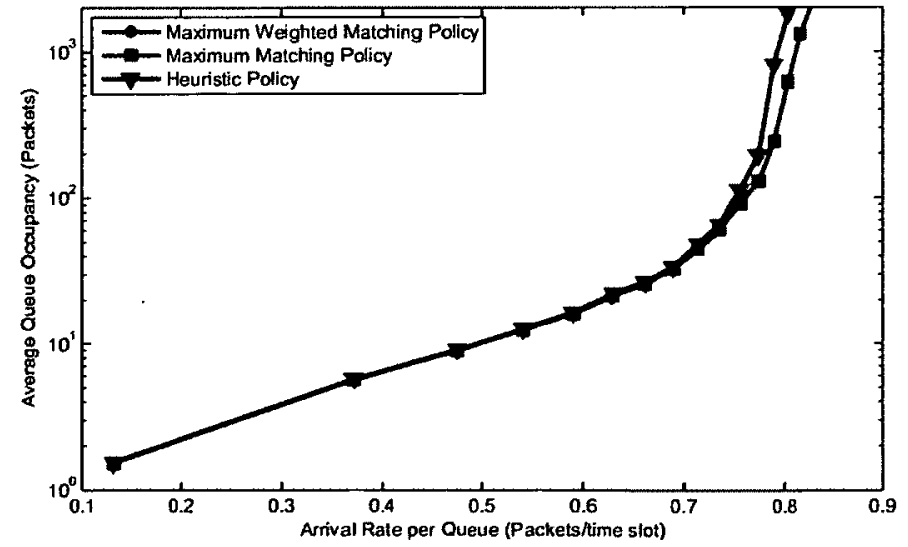

(c) $p=0.8$

Figure 5.4: Average total queue occupancy, $N=8, K=8, q=0.8$ 


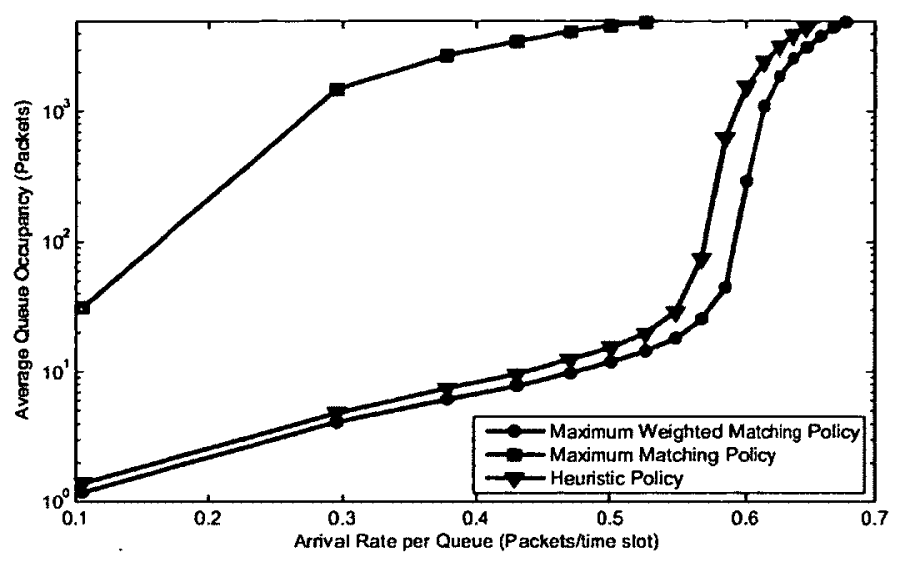

(a) $q=0.8$

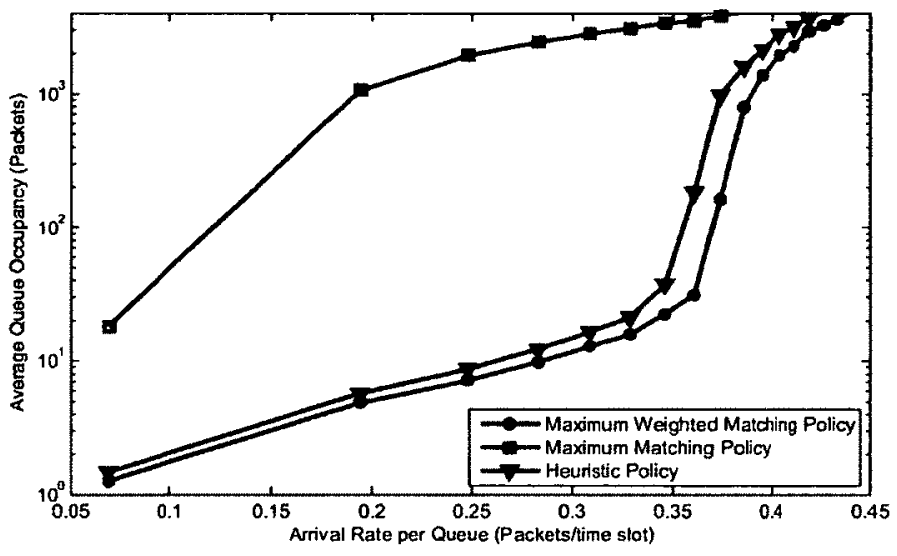

(b) $q=0.5$

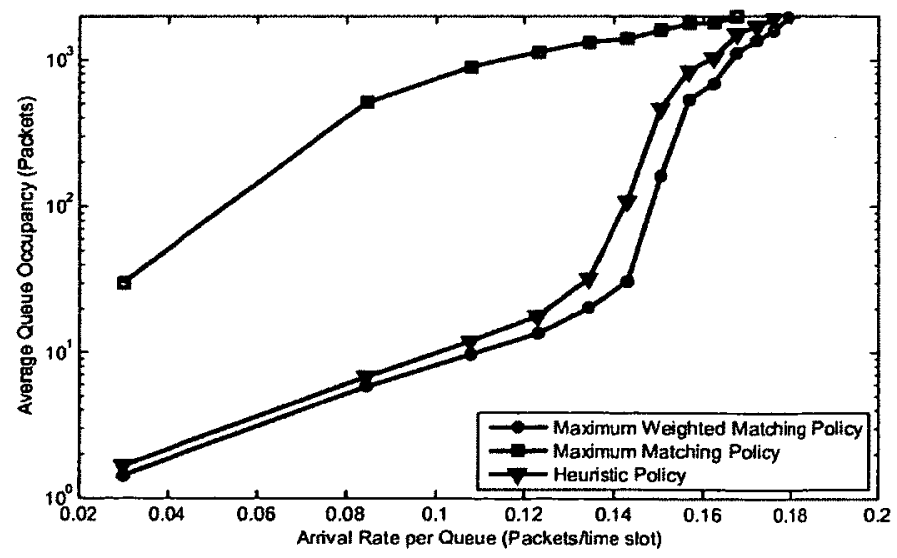

(c) $q=0.2$

Figure 5.5: Average total queue occupancy, $N=8, K=6, p=0.5$ 


\section{Chapter 6}

\section{Conclusions and Suggestions for Future Research}

\subsection{Conclusions}

In this thesis, we focused on the stability analysis and stochastic control of a series of multi-server queueing systems with time varying channel condition. More specifically, our contribution in the stability analysis of these systems was to derive an explicit characterization of the stability region for each system. First, in Chapter 3 , we considered this problem for three multi-server queueing systems with random connectivities between the queues and the servers, namely, "multi-interface queueing system with a randomly connected server bank", "multi-queue multi-server system with random connectivities" and "multi-server single-interface queueing system with random connectivities". These queueing models can be applied to study stochastic control problems in realistic wireless networks. For each system, we derived the necessary and sufficient conditions for the stability of the system for general arrival processes with bounded second moment. We then showed that for stationary packet arrival processes, these conditions define the system stability region. We further obtained an upper bound, in an explicit form, for the average queue occupancy of each system. Using simulations, we showed that the obtained bounds are not tight.

In Chapter 4, the multi-queue multi-server system (studied in Chapter 3) was generalized to support a more general stationary channel distribution. For such a system, it has been proven that Maximum Weight policy is the throughput optimal policy. We focused on the stability region characterization problem for this model. We first showed that the network stability region (capacity region) is a polytope in 
$\mathbb{R}_{+}^{N}$. For the stability region polytope, all the coefficients associated to the facetdefining hyperplanes of the stability region polytope were determined explicitly. An upper bound (in an explicit form) was also obtained for the average queueing delay of Maximum Weight (MW) server allocation policy. We also considered the stability region for the fluid model MQMS. In the fluid model MQMS system, the channel capacity and arrival processes are assumed to be continuous random variables. For the fluid model MQMS, the stability region was determined by an infinite set of linear inequalities given by (4.32). This agrees with intuition as in this case, the stability region is characterized by a convex surface instead of a convex polytope. By use of an example, it was shown that depending on the channel distribution and the number of queues, we may characterize the stability region by a finite set of non-linear inequalities instead of infinite number of linear inequalities.

Finally, we reconsidered the multi-server single-interface model in Chapter 5 with the objective of minimizing the average queueing delay in the system. Note that in Chapter 3 this system was studied from the stability point of view and it was noted that Maximum Weighted Matching (MWM) policy is a throughput-optimal server assignment policy for this system. In Chapter 5, we proved that the MWM policy not only is throughput-optimal, but also is delay-optimal for a symmetric system. Specifically, we demonstrated that for a symmetric system with i.i.d. Bernoulli packet arrivals and connectivities, MWM minimizes, in stochastic ordering sense, a broad range of cost functions of the queue lengths including total queue occupancy (or equivalently minimizes the average queueing delay). Then, we extended the model by considering imperfect services where it is assumed that the service of a scheduled packet fails randomly with a certain probability. We proved that the same policy is still optimal for the extended model. We also showed that the results are still valid for some extended connectivity and arrival processes (e.g., Poisson arrival process). Simulations were conducted to compare the performance of the MWM policy with some other sub-optimal policies.

\subsection{Future Directions}

There are many interesting open problems and research directions yet to be addressed in this area of research. In the following, a number of these directions which are considered to be of significant theoretical and practical impact are highlighted. 
- The stability analysis for the multi-interface queueing system with a randomly connected server bank and the multi-server single-interface queueing system with random connectivities were performed in Chapter 3 just for a system with ON-OFF channels. A possible research direction which is of particular interest both theoretically and practically would be to generalize the analysis for a system with $M$-ary channels.

- Note that characterizing the explicit form of the stability region is particularly important as it enables us to derive a closed form expression for the average queueing delay bound. Using the explicit from of the stability region, we derived the delay bound and compared it to the system actual performance for the three queueing systems studied in Chapter 3. We observed that the obtained delay bound is not tight. Therefore, a possible future direction in this area could be further sharpen the bound by applying more sophisticated stability analysis tools.

- In Chapter 4, the stability region of a general fluid flow MQMS system was characterized by an infinite number of linear inequalities. By using an example we further showed that depending on the channel distribution the stability region may be characterized by a finite number of non-linear inequalities. However, the general problem of stability region characterization for the fluid model MQMS can be considered as a possible future research problem.

- In most of the problems that has been considered in the literature, the studied network was assumed to employ pure packet forwarding. In other words, the nodes are not doing any processing on the transmitted and received data packets. Network coding is a new alternative approach that was invented in 2000 [93]. In network coding enabled systems, in contrast to simple store and forward routing the nodes have the ability to process the received packets and possibly combine them before transmission. There have been a lot of efforts in the literature to study network coding from different perspectives (e.g., information theory, combinatorial theory, coding theory, etc.). However, little research work has been reported in the area of stochastic optimization in network coding enabled systems where the main objective is to investigate optimal cross-layer 
policies that optimize a long term performance attribute of the system, especially queuing delay (which is a big challenge in wireless systems). More specifically, one important future direction for this thesis could be to apply similar queueing models to network coding enabled wireless systems and investigate for delay optimal multicasting policies in such networks. The analysis may be further extended to multi-hop systems with the objective of determining the delay optimal joint resource scheduling and network coding in multi-hop wireless systems. 


\section{List of References}

[1] L. Georgiadis, M. J. Neely, and L. Tassiulas, Resource Allocation and Cross Layer Control in Wireless Networks. Now Publisher, 2006.

[2] X. Lin, N. B. Shroff, and R. Srikant, "A tutorial on cross-layer optimization in wireless networks," IEEE Journal on Selected Areas in Communications, vol. 24, pp. 1452-1463, August 2006.

[3] L. Tassiulas and A. Ephremides, "Dynamic server allocation to parallel queues with randomly varying connectivity," IEEE Trans. Inform. Theory, vol. 39, pp. 466-478, Mar. 1993.

[4] L. Tassiulas and A. Ephremides, "Stability properties of constrained queueing systems and scheduling policies for maximum throughput in multihop radio networks," IEEE Trans. Auto. Control, vol. 37, pp. 1936-1949, Dec. 1992.

[5] K. Kar, X. Luo, and S. Sarkar, "Throughput-optimal scheduling in multichannel access point networks under infrequent channel measurements," IEEE Trans. Wireless Comm., vol. 7, pp. 2619-2629, July 2008.

[6] N. McKeown, A. Mekkittikul, V. Anantharam, and J. Walrand, "On achieving $100 \%$ throughput in an input-queued switch," IEEE Trans. Commun., vol. 47, pp. 1260-1272, Aug. 1999.

[7] L. Tassiulas, "Scheduling and performance limits of networks with constantly changing topology," IEEE Trans. Inform. Theory, vol. 43, pp. 1067-1073, May 1997.

[8] L. Tassiulas, "Linear complexity algorithms for maximum throughput in radio networks and input queued switches," in Proc. of IEEE INFOCOM, (San Francisco, CA, USA), Apr. 1998.

[9] M. J. Neely and E. Modiano, "Logarithmic delay for nxn packet switches," in Proc. of IEEE Workshop on High Performance Switching and Routing, (Phoenix, AZ, USA), Apr. 2004.

[10] E. Leonardi, M. Melia, F. Neri, and M. A. Marson, "Bounds on average delays and queue size averages and variances in input-queued cell-based switches," in Proc. of IEEE INFOCOM 2001, (Anchorage, Alaska, USA), Apr. 2001. 
[11] S. Kittipiyakul and T. Javidi, "A fresh look at optimal subcarrier allocation in ofdma systems," in Proc. IEEE Conference on Decision and Control, (Atlantis, Paradise Island, Bahamas), Dec. 2004.

[12] S. Kittipiyakul and T. Javidi, "Resource allocation in ofdma with time-varying channel and bursty arrivals," IEEE Commun. Lett., vol. 11, pp. 708-710, Sep. 2007.

[13] T. Javidi, "Rate stable resource allocation in ofdm systems: from waterfilling to queue-balancing," in Proc. Allerton Conference on Communication, Control, and Computing, Oct. 2004.

[14] H. Halabian, I. Lambadaris, and C.-H. Lung, "Network capacity region of multiqueue multi-server queueing system with time varying connectivities," in Proc. of IEEE Int. Symp. on Inform. Theory (ISIT'10), (Austin, TX, USA), June 2010.

[15] H. Halabian, I. Lambadaris, and C.-H. Lung, "On the stability region of multiqueue multi-server queueing systems with stationary channel distribution," in Proc. of IEEE Int. Symp. on Inform. Theory (ISIT'11), (Saint Petersburg, Russia), Aug. 2011.

[16] M. J. Neely, Dynamic power allocation and routing for satellite and wireless networks with time varying channels. PhD thesis, Massachusetts Institute of Technology, LIDS, 2003.

[17] M. J. Neely, E. Modiano, and C. E. Rohrs, "Dynamic power allocation and routing for time varying wireless networks," IEEE Journal on Selected Areas in Communications, Special Issue on Wireless Ad-hoc Networks, vol. 23, pp. 89-103, Jan. 2005.

[18] H. Halabian, I. Lambadaris, C.-H. Lung, and A. Srinivasan, "Throughputoptimal relay selection in multiuser cooperative relaying networks," in Proc. IEEE MILCOM 2010, (San Jose, CA, USA), Nov. 2010.

[19] J.-H. Chang and L. Tassiulas, "Routing for maximum system lifetime in wireless ad-hoc networks," in Proc. Allerton Conference on Communication, Control, and Computing, Sep. 1999.

[20] J.-H. Chang and L. Tassiulas, "Energy conserving routing in wireless ad-hoc networks," in Proc. IEEE INFOCOM 2000, (Tel-Aviv, Israel), Mar. 2000.

[21] C. Chen and D. Lee, "A joint design of distributed qos scheduling and power control for wireless networks," in Proc. IEEE INFOCOM'06, (Barcelona, Spain), Apr. 2006.

[22] Y. Wei, F. Yu, M. Song, and V. Leung, "Energy efficient distributed relay selection in wireless cooperative networks with finite state markov channels," in Proc. IEEE Globecom'09, (Honolulu, HI, USA), Dec. 2009.

[23] M. J. Neely, E.Modiano, and C.-P. Li, "Fairness and optimal stochastic control for heterogeneous networks," in Proc. of IEEE INFOCOM'05, (Miami, FL, USA), Mar. 2005. 
[24] M. J. Neely, "Energy optimal control for time varying wireless networks," IEEE Trans. Inform. Theory, vol. 52, pp. 2915-2934, July 2006.

[25] R. Berry and R. Gallager, "Communication over fading channels with delay constraints," IEEE Trans. Inform. Theory, vol. 48, pp. 1135-1149, May 2002.

[26] L. Massouliii $£_{j}$ and J. Roberts, "Bandwidth sharing: Objectives and algorithms," IEEE/ACM Transactions on Networking, vol. 10, pp. 320-328, June 2002.

[27] B. Radunovic and J. L. Boudec, "Rate performance objectives of multihop wireless networks," IEEE Transactions on Mobile Computing, vol. 3, pp. 334-349, December 2004.

[28] L. Xiao, M. Johansson, and S. P. Boyd, "Simultaneous routing and resource allocation via dual decomposition," IEEE Transactions on Communications, vol. 52, pp. 1136-1144, July 2004.

[29] M. Chiang, "Balancing transport and physical layer in wireless multihop networks: Jointly optimal congestion control and power control," IEEE Journal on Selected Areas in Communications, vol. 23, pp. 104-116, January 2005.

[30] A. Stolyar, "Greedy primal-dual algorithm for dynamic resource allocation in complex networks," Queueing Systems, vol. 54, pp. 203-220, November 2006.

[31] X. Lin and N. B. Shroff, "Joint rate control and scheduling in multihop wireless networks," in Proc. of IEEE Conference on Decision and Control, (Atlantis, Paradise Island, Bahamas), Dec. 2004.

[32] R. L. Cruz and A. V. Santhanam, "Optimal routing, link scheduling and power control in multi-hop wireless networks," in Proc. of IEEE INFOCOM 2003, (San Francisco, CA, USA), Apr. 2003.

[33] J. W. Lee, R. R. Mazumdar, and N. B. Shroff, "Downlink power allocation for multi-class cdma wireless networks," in Proc. IEEE INFOCOM 2002, (New York, USA), Jun. 2002.

[34] L. Xiao, M. Johansson, and S. P. Boyd, "Simultaneous routing and resource allocation for wireless networks," in Proc. of the 39th Annual Allerton Conf. on Comm., Control, Comput., Oct. 2001.

[35] A. Eryilmaz, "Fair resource allocation in wireless networks using queue-lengthbased scheduling and congestion control," in Proc. of IEEE INFOCOM'05, (Miami, FL, USA), Mar. 2005.

[36] G. S. Kasbekar, Y. Bejerano, and S. Sarkar, "Lifetime and coverage guarantees through distributed coordinate-free sensor activation," in Proc. of ACM MobiCom, (Beijing, China), Sep. 2009.

[37] H. Halabian, R. Changiz, F. Yu, I. Lambadaris, and H. Tang, "Optimal reliable relay selection in multiuser cooperative relaying networks," $A C M / S p r i n g e r$ Wireless Networks, vol. 18, pp. 591-603, August 2012. 
[38] M. J. Neely, Stochastic Network Optimization with Application to Communication and Queueing Systems. Morgan \& Claypool, 2010.

[39] M. J. Neely, "Super-fast delay tradeoffs for utility optimal fair scheduling in wireless networks," IEEE Journal on Selected Areas in Communications (JSAC), Special Issue on Nonlinear Optimization of Communication Systems, vol. 24, pp. 1489-1501, August 2006.

[40] T. Bonald, "Throughput performance in networks with linear capacity constraints," in Proc. of 40th Annual Conference on Information Sciences and Systems, (Princeton, NJ, USA), Mar. 2006.

[41] A. Eryilmaz and R. Srikant, "Asymptotically tight steady-state queue length bounds implied by drift conditions." [Online]. Available: http://arxiv.org/abs/1104.0327, April 2011.

[42] M. J. Neely, "Delay analysis for max weight opportunistic scheduling in wireless systems," in Proc. 46th Annual Allerton Conf. on Communication, Control, and Computing, Sep. 2008.

[43] M. J. Neely, "Delay analysis for maximal scheduling in wireless networks with bursty traffic," in Proc. IEEE INFOCOM 2008, (Phoenix, AZ, USA), Apr. 2008.

[44] N. Bambos and G. Michailidis, "On the stationary dynamics of parallel queues with random server connectivities," in Proc. of IEEE Conference on Decision and Control, (New Orleans, LA), Dec. 1995.

[45] N. Bambos and G. Michailidis, "On parallel queueing with random server connectivity and routing constraints," Probability in Engineering and Informational Sciences, vol. 16, pp. 185-203, 2002.

[46] V. Tsibonis, L. Georgiadis, and L. Tassiulas, "Exploiting wireless channel state information for throughput maximization," IEEE Transactions on Information Theory, vol. 50, pp. 2566-2582, November 2004.

[47] S. Asmussen, Applied Probability and Queues. New York: Spring-Verlag, 2003.

[48] F. Baccelli and P. Bremaud, Elements of Queueing Theory 2nd ed. Berlin: Springer, 2003.

[49] M. El-Taha and S. S. Jr., Sample-Path Analysis of Queueing Systems. Boston: Kluwer Academic Publishers, 1999.

[50] B. Rong and A. Ephremides, "Protocol-level cooperation in wireless networks: Stable throughput and delay analysis," in Proc. of the 7th international conference on Modeling and Optimization in Mobile, Ad Hoc, and Wireless Networks (WiOPT'09), (Seoul, Korea), Jun. 2009.

[51] B. Rong and A. Ephremides, "Cooperation above the physical layer: The case of a simple network," in Proc. of IEEE International Symposium on Information Theory (ISIT'O9), (Seoul, Korea), Jul. 2009. 
[52] N. Pappas, A. Traganitis, and A. Ephremides, "Stability and performance issues of a relay assisted multiple access scheme," in Proc. of GLOBECOM 2010, (Miami, FL, USA), Dec. 2010.

[53] A. E. A. Fanous, "Network-level cooperative protocols for wireless multicasting: Stable throughput analysis and use of network coding," in Proc. of Information Theory Workshop (ITW'10), (Dublin, Ireland), Sep. 2010.

[54] O. Simeone, Y. Bar-Ness, and U. Spagnolini, "Stable throughput of cognitive radios with and without relaying capability. communications," IEEE Transactions on Communications, vol. 55, pp. 2351-2360, December 2007.

[55] S. Ghez, S. Verdu, and S. Schwartz, "Stability properties of slotted aloha with multipacket reception capability," IEEE Transactions on Automatic Control, vol. 33, pp. 640-649, July 1988.

[56] G. del Angel and T. L. Fine, "Optimal power and retransmission control policies for random access systems," IEEE/ACM Trans. Netw., vol. 12, pp. 1156-1166, December 2004.

[57] V. Naware, G. Mergen, and L. Tong, "Stability and delay of finite-user slotted aloha with multipacket reception," IEEE Transactions on Information Theory, vol. 51, pp. 2636-2656, July 2005.

[58] R. Rao and A. Ephremides, "On the stability of interacting queues in a multiaccess system," IEEE Transactions on Information Theory, vol. 34, pp. 918-930, September 1988.

[59] W. Szpankowski, "Stability conditions for some distributed systems: Buffered random access systems," Advances in Applied Probability, vol. 26, pp. 498-515, June 1994.

[60] W. Luo and A. Ephremides, "Stability of $\mathrm{n}$ interacting queues in random-access systems," IEEE Transactions on Information Theory, vol. 45, pp. 1579-1587, July 1999.

[61] M. J. Neely, "Optimal energy and delay tradeoffs for multi-user wireless downlinks," IEEE Trans. Inform. Theory, vol. 53, pp. 3095-3113, September 2007.

[62] A. Ganti, E. Modiano, and J. N. Tsitsiklis, "Optimal transmission scheduling in symmetric communication models with intermittent connectivity," IEEE Trans. Inform. Theory, vol. 53, pp. 998-1008, Mar. 2007.

[63] H. Al-Zubaidy, I. Lambadaris, and I. Viniotis, "Optimal resource scheduling in wireless multi-service systems with random channel connectivity," in Proc. of IEEE Global Communications Conference (GLOBECOM 2009), (Honolulu, HI, USA), Nov. 2009.

[64] H. Halabian, I. Lambadaris, and C.-H. Lung, "Dynamic channel and interface management in multi-channel multi-interface wireless access networks," in IEEE Global Communications Conference (IEEE Globecom'10), (Miami, FL, USA), Dec. 2010. 
[65] S. Kittipiyakul and T. Javidi, "Delay-optimal server allocation in multi-queue multi-server systems with time-varying connectivities," IEEE Trans. Inform. Theory, vol. 55, pp. 2319-2333, May 2009.

[66] H. Halabian, I. Lambadaris, and C.-H. Lung, "Delay optimal server assignment to symmetric parallel queues with random connectivities," in Proc. of 50th IEEE Conference on Decision and Control and European Control Conference (CDCECC 2011), (Orlando, FL, USA), Dec. 2011.

[67] H. Halabian, I. Lambadaris, and C.-H. Lung, "Optimal server assignment in multi-server parallel queueing systems with random connectivities and random service failures," in Proc. of IEEE International Conference on Communications (ICC 2012), (Ottawa, ON, Canada), Jun. 2012.

[68] H. Halabian, I. Lambadaris, and C.-H. Lung, "Optimal server assignment in multi-server queueing systems with random connectivities," Submitted to IEEE/ACM Transactions on Networking, 2011.

[69] H. Halabian, I. Lambadaris, and C.-H. Lung, "Explicit characterization of stability region for stationary multi-queue multi-server systems," Submitted to IEEE Transactions on Automatic Control, 2011.

[70] H. Halabian, I. Lambadaris, and C.-H. Lung, "Stability analysis of randomly connected multi-server queueing systems," Submitted to Stochastic Models, 2012.

[71] D. N. Tse, "Optimal power allocation over parallel broadcast channels," in Proc. of International Symposium on Information Theory, (Ulm, Germany), Jun. 1997.

[72] S. Borst, "User-level performance of channel-aware scheduling algorithms in wireless data networks," IEEE/ACM Transactions on Networking, vol. 13, pp. 636647 , June 2005.

[73] R. Berry, P. Liu, and M. Honig, "Design and analysis of downlink utility-based schedulers," in Proc. of the 40th Allerton Conference on Communication, Control and Computing, Oct. 2002.

[74] F. Kelly, A. Maulloo, and D. Tan, "Rate control for communication networks: Shadow prices, proportional fairness, and stability," Journal of the Operational Research Society, vol. 49, pp. 237-252, Mar. 1998.

[75] F. Kelly, "Charging and rate control for elastic traffic," European Transactions on Telecommunications, vol. 8, pp. 33-37, Jan. 1997.

[76] A. L. Stolyar, "Maximizing queueing network utility subject to stability: Greedy primal-dual algorithm," Queueing Systems: Theory and Applications, vol. 50, pp. 401-457, Aug. 2005.

[77] D. Julian, M. Chiang, D. O'Neil, and S. Boyd, "Qos and fairness constrained convex optimization of resource allocation for wireless cellular and ad-hoc networks," in Proc. of IEEE INFOCOM'02, (New York City, USA), June 2002. 
[78] T. Rappaport, Wireless Communications: Principles and Practice, 2nd ed. NJ, USA: Prentice Hall, 2001.

[79] A. Goldsmith, Wireless Communications. NY, USA: Cambridge University Press, 2005.

[80] V. Raman and N. H. Vaidya, "Adjacent channel interference reduction in multichannel wireless networks using intelligent channel allocation," tech. rep., University of Illinois at Urbana-Champaign, 2009.

[81] V. Bhandari and N. H. Vaidya, "Scheduling in multi-channel wireless networks with limited information," tech. rep., University of Illinois at Urbana-Champaign, 2008.

[82] V. Bhandari and N. H. Vaidya, "Scheduling in multi-channel wireless networks," in 11th International Conference on Distributed Computing \& Networking (ICDNC 2010), (Kolkata, India), Jan. 2010.

[83] S. Merlin, N. Vaidya, and M. Zorzi, "Resource allocation in multi-radio multichannel multi-hop wireless networks," in IEEE INFOCOM'08, (Phoenix, AZ, USA), Apr. 2008.

[84] M. J. Neely, "Order optimal delay for opportunistic scheduling in multi-user wireless uplinks and downlinks," IEEE/ACM Transactions on Networking, vol. 16, pp. 1188-1199, October 2008.

[85] A. Schrijver, Theory of Linear and Integer Programming. Chichester: John Wiley and Sons, 1986.

[86] J. Lee, A First Course in Combinatorial Optimization. Cambridge, UK: Cambridge Texts in Applied Mathematics, Cambridge University Press, 2004.

[87] H. W. Kuhn, "The hungarian method for the assignment problem," Naval Research Logistic Quarterly, pp. 2:83-97, 1955.

[88] A. Leon-Garcia, Probability, Statistics, and Random Processes For Electrical Engineering. P. Prentice Hall, 2008.

[89] H. Al-Zubaidy, I. Lambadaris, and I. Viniotis, "Optimal multi-server allocation to parallel queues with independent random queue-server connectivity." [Online]. Available: http://arxiv.org/abs/1103.1448, April 2011.

[90] D. Stoyan, Comparison Methods for Queues and other Stochastic Models. Chichester: J. Wiley and Sons, 1983.

[91] S. M. Ross, Stochastic Processes, 2nd ed. New York: J. Wiley and Sons, 1996.

[92] R. Lidl and G. Pilz, Applied abstract algebra, 2nd edition. New York: Springer, 1998.

[93] R. Ahlswede, N. Cai, S. yen Robert Li, and R. W. Yeung, "Network information flow," IEEE Transactions on Information Theory, vol. 46, pp. 1204-1216, July 2000 . 


\section{Appendix A}

\section{Mathematical Background}

\section{A.1 Lyapunov Drift}

A very important and useful mathematical tool used in network stability analysis and stochastic control/optimization of wireless networks is the Lyapunov Drift technique introduced in $[1,6,10,16,17]$. The main idea behind the Lyapunov stability method is to define a nonnegative function of queue backlogs which can be seen as a measure of the total aggregated backlog in the system at time slot $t$. Then, we evaluate the "drift" of such function in two successive time slots by taking the effect of control decisions (scheduling or resource allocation policy) into account. If the expected value of the drift is negative as the backlog goes beyond a fixed threshold, then the system is stable. This method was used in $[1,6,10,16,17]$ to prove the stability of a number of queueing systems.

For a queueing system with $N$ queues and queue length vector $X(t)=$ $\left(X_{1}(t), \ldots, X_{N}(t)\right)$, the following quadratic function has been frequently used in literature $([1,6,10,16,17])$ as a Lyapunov function.

$$
L(X(t))=\sum_{n=1}^{N} X_{n}^{2}(t)
$$

Assume that $E\left[X_{n}(0)\right]<\infty, \forall n \in \mathcal{N}$ and $X(t)$ evolves with some probabilistic law (not necessarily Markovian). Then, the following holds [1].

Lemma A.1 If there exist constants $B>0$ and $\delta>0$ such that for all time slots $t$ 
we have

$$
E[L(X(t+1))-L(X(t)) \mid X(t)] \leq B-\delta \sum_{n=1}^{N} X_{n}(t)
$$

then the system is strongly stable and further we have

$$
\limsup _{t \rightarrow \infty} \frac{1}{t} \sum_{\tau=0}^{t-1} \sum_{n=1}^{N} E\left[X_{n}(\tau)\right] \leq \frac{B}{\delta}
$$

The proof of the lemma can be found in $[1,16]$. The left hand side of expression (A.2) is usually called Lyapunov drift function which is a measure of the expected value of changes in the backlog in two successive time slots. From Lemma A.1, the idea behind Lyapunov method in stabilizing queueing systems can be easily observed. It is not hard to show that, when the aggregated backlog in the system goes beyond the bound $\frac{B}{\delta}$, the Lyapunov drift in the left hand side of (A.2) will be negative, meaning that the system receives a negative drift on the expected aggregated backlog in two successive time slots. In other words, the system tends toward lower backlogs and this results in its stability.

\section{A.2 Fundamental Concepts of Polytopes}

In this section, a brief review on convex polytopes and their fundamental properties is provided. These concepts will be needed for specifying the stability region of the MQMS system in Section 4.2.

Definition A.1 A convex polytope is defined as the convex hull of a finite set of points $[85,86]$.

According to Weyl's Theorem [86], a polytope in $\mathbb{R}^{N}$ always can be expressed by a set $\mathcal{P}=\left\{x \in \mathbb{R}^{N} \mid \alpha_{\ell} x^{\mathrm{T}} \leq \beta_{\ell}\right.$ for $\left.\ell=1,2, \ldots, L\right\}$ for some positive integer $L$ and $\alpha_{\ell} \in \mathbb{R}^{N}$ and $\beta_{\ell} \in \mathbb{R}$.

Definition A.2 The dimension of a polytope $\mathcal{P}$ is represented by $\operatorname{dim}(\mathcal{P})$ and is defined as one less than the maximum number of affinely independent points in $\mathcal{P}$ $[85,86]$. 
A polytope $\mathcal{P} \subset \mathbb{R}^{N}$ is said to be full dimensional if $\operatorname{dim}(\mathcal{P})=N$.

Dimension Theorem: For a polytope $\mathcal{P} \subset \mathbb{R}^{N}$, dimension of $\mathcal{P}$ is equal to $N$ minus the maximum number of linearly independent equations satisfied by all the points in $\mathcal{P}$.

Definition A.3 For given $a$ and $b$, inequality $a x^{\mathrm{T}} \leq b$ is called valid for polytope $\mathcal{P}$ if for every point $x_{0} \in \mathcal{P}, a x_{0}^{\mathrm{T}} \leq b$.

Definition A.4 A face of polytope $\mathcal{P}$ is defined as $\mathcal{F}=\left\{x \in \mathcal{P} \mid a x^{\mathrm{T}}=b\right\}$ where inequality $a x^{T} \leq b$ is a valid inequality for $\mathcal{P}$.

A valid equality $a x_{0}^{\mathrm{T}}=b$ is called a face-defining hyperplane for $\mathcal{P}$ if its associated face is not empty. Therefore, $a x_{0}^{\mathrm{T}}=b$ is a face defining hyperplane for $\mathcal{P}$ if it intersects with $\mathcal{P}$ at least at one point. Note that $\mathcal{P}$ has finitely many faces. However, the face defining hyperplanes of a polytope can be infinite.

Definition A.5 A facet of polytope $\mathcal{P}$ is a maximal face distinct ${ }^{1}$ from $\mathcal{P}$ [85]. Hence, all faces of $\mathcal{P}$ with dimension $\operatorname{dim}(\mathcal{P})-1$ are called facets of $\mathcal{P}$.

For a polytope $\mathcal{P}=\left\{x \in \mathbb{R}^{N} \mid \alpha_{\ell} x^{\mathrm{T}} \leq \beta_{\ell}\right.$ for $\left.\ell=1,2, \ldots, L\right\}$ an inequality is redundant if polytope $\mathcal{P}$ remains unchanged by removing the inequality.

Redundancy Theorem in Polytopes [86]: Face defining hyperplanes describing faces of dimension less than $\operatorname{dim}(\mathcal{P})-1$ are redundant.

Redundancy theorem states that to describe a polytope completely, only facet defining hyperplanes are sufficient.

\footnotetext{
${ }^{1}$ Maximal relative to inclusion
} 


\section{Appendix B}

\section{Proofs of the Results of Chapter 3}

\section{B.1 Proof of Lemma 3.1}

Proof: If we write equation (3.4) for $\tau=1,2, \ldots, t$ and then add them up, we will have

$$
X_{n}(t)=X_{n}(0)-\sum_{\tau=1}^{t} H_{n}(t)+\sum_{\tau=1}^{t} A_{n}(t)
$$

Taking the expectation of both sides, dividing by $t$ and then taking the limit as $t$ goes to infinity, we will have the following.

$$
\lim _{t \rightarrow \infty} \frac{E\left[X_{n}(t)\right]}{t}=\lim _{t \rightarrow \infty} \frac{E\left[X_{n}(0)\right]}{t}-\lim _{t \rightarrow \infty} \frac{1}{t} \sum_{\tau=1}^{t} E\left[H_{n}(t)\right]+\lim _{t \rightarrow \infty} \frac{1}{t} \sum_{\tau=1}^{t} E\left[A_{n}(t)\right]
$$

According to Lemma 3.1 in [1] and the assumption that $E\left[X_{n}(0)\right]<\infty$, the left hand side term and the first term in the right hand side term are equal to zero and therefore the result is proven.

\section{B.2 Proof of Lemma 3.2}

Proof: For a stable system, similar to what we proved in Lemma 3.4 we can prove that for any subset of queues $Q \subseteq \mathcal{N}$, the average expected arrival is equal to the 
average expected departure, i.e.,

$$
\lim _{t \rightarrow \infty} \frac{1}{t} \sum_{\tau=1}^{t} \sum_{n \in Q} E\left[A_{n}(\tau)\right]=\lim _{t \rightarrow \infty} \frac{1}{t} \sum_{\tau=1}^{t} \sum_{n \in Q} E\left[H_{n}(\tau)\right]
$$

Suppose that events $B_{i}^{Q}$ are defined as $B_{i}^{Q}=\{$ exactly $i$ queues of (sub)set $Q$ are connected $\}$. By conditioning the expectation in the right hand side of (B.3) to the number of connected queues at time slot $\tau$, we have

$$
\sum_{n \in Q} E\left[H_{n}(\tau)\right]=\sum_{i=1}^{|Q|} E\left[\sum_{n \in Q} H_{n}(\tau) \mid B_{i}^{Q}\right] P\left(B_{i}^{Q}\right)
$$

Since $E\left[\sum_{n \in Q} H_{n}(\tau) \mid B_{i}^{Q}\right] \leq \min \{K, i R\}$, (B.4) can be bounded by

$$
\sum_{n \in Q} E\left[H_{n}(\tau)\right] \leq \sum_{i=1}^{|Q|} \min \{K, i R\} P\left(B_{i}^{Q}\right)
$$

Note that $P\left(E_{i}^{Q}\right)=\sum_{j=i}^{|Q|} P\left(B_{j}^{Q}\right)$ and therefore

$$
\begin{gathered}
P\left(B_{j}^{Q}\right)=P\left(E_{j}^{Q}\right)-P\left(E_{j+1}^{Q}\right) \quad \text { for } \quad 1 \leq j<|Q|-1 \\
P\left(B_{|Q|}^{Q}\right)=P\left(E_{|Q|}^{Q}\right)
\end{gathered}
$$

By replacing (B.6) in (B.5) we have

$$
\begin{aligned}
\sum_{n \in Q} E\left[H_{n}(\tau)\right] & \leq \sum_{i=1}^{|Q|-1} \min \{K, i R\}\left(P\left(E_{i}^{Q}\right)-P\left(E_{i+1}^{Q}\right)\right)+\min \{K,|Q| R\} P\left(E_{|Q|}^{Q}\right) \\
& =\sum_{i=1}^{|Q|}(\min \{K, i R\}-\min \{K,(i-1) R\}) P\left(E_{i}^{Q}\right) \\
& =\sum_{i=1}^{|Q|} \min \left\{(K-(i-1) R)^{+}, R\right\} P\left(E_{i}^{Q}\right)
\end{aligned}
$$

Noting (B.3) and (B.7) the lemma follows. 


\section{B.3 Proof of Lemma 3.3}

Proof: We will start with the Lyapunov drift evaluation and use the quadratic function $L(X(t))=\sum_{n=1}^{N} X_{n}^{2}(t)$ as our Lyapunov function. The Lyapunov drift for two successive time slots has the following form.

$$
\begin{aligned}
& E[L(X(t+1))-L(X(t)) \mid X(t))] \\
& \quad=E\left[\sum_{n=1}^{N}\left(X_{n}(t+1)-X_{n}(t)\right)^{2} \mid X(t)\right]+2 E\left[\sum_{n=1}^{N} X_{n}(t)\left(X_{n}(t+1)-X_{n}(t)\right) \mid X(t)\right]
\end{aligned}
$$

For the the first term we have

$$
\begin{aligned}
& E\left[\sum_{n=1}^{N}\left(X_{n}(t+1)-X_{n}(t)\right)^{2} \mid X(t)\right] \\
& \quad \leq E\left[\sum_{n=1}^{N} A_{n}^{2}(t+1) \mid X(t)\right]+E\left[\sum_{n=1}^{N} H_{n}^{2}(t) \mid X(t)\right] \leq N\left(A_{\text {max }}^{2}+R^{2}\right)
\end{aligned}
$$

where the last inequality comes from the fact that $0 \leq H_{n}(t) \leq R$. For the second term in (B.8) we have

$$
\begin{aligned}
E & {\left[\sum_{n=1}^{N} X_{n}(t)\left(X_{n}(t+1)-X_{n}(t)\right) \mid X(t)\right] } \\
& =E\left[\sum_{n=1}^{N} X_{n}(t) A_{n}(t+1) \mid X(t)\right]-E\left[\sum_{n=1}^{N} X_{n}(t) H_{n}(t+1) \mid X(t)\right]
\end{aligned}
$$

Before we proceed further, we need to introduce some new notation. We sort the queue length vector at time slot $t$ in an ascending order $X_{s_{1}}, X_{s_{2}}, \ldots, X_{s_{N}}$, i.e. $X_{s_{n}}(t) \geq$ $X_{s_{n-1}}(t)$ for all $n=2, \ldots, N$ and if $X_{s_{n}}(t)=X_{s_{n-1}}(t)$, then $s_{n} \geq s_{n-1}$. We will denote the queues corresponding to these queue lengths by $Q_{s_{1}}, Q_{s_{2}}, \ldots, Q_{s_{N}}$. The first term in (B.10) can be written as follows.

$$
\begin{aligned}
E[ & \left.\sum_{n=1}^{N} X_{n}(t) A_{n}(t+1) \mid X(t)\right]=\sum_{l=1}^{N} E\left[A_{s_{l}}(t+1)\right] X_{s_{l}}(t+1) \\
& =\sum_{j=2}^{N}\left(X_{s_{j}}(t)-X_{s_{j-1}}(t)\right) \sum_{l=j}^{N} E\left[A_{s_{l}}(t+1)\right]+X_{s_{1}}(t) \sum_{l=1}^{N} E\left[A_{s_{l}}(t+1)\right]
\end{aligned}
$$


Furthermore, consider the following events of the connectivity processes at time $t+1$. Assume that for $1 \leq i<j \leq N+1$ we define event $\mathcal{Z}_{i j}(t+1)$ as

$$
\mathcal{Z}_{i j}(t+1)=\left\{C_{s_{i}}(t+1)=1, C_{s_{\ell}}(t+1)=0 \text { for } i<\ell<j\right\}
$$

and $\mathcal{Z}_{0 j}(t+1)$ for $1<j \leq N+1$ as

$$
\mathcal{Z}_{0 j}(t+1)=\left\{C_{s_{\ell}}(t+1)=0 \text { for } \ell<j\right\}
$$

Before we elaborate on the second term of (B.10) we need to prove the following claims.

Claim 1: For LCQ policy at each time slot $t$ we have

$$
E\left[\sum_{n=1}^{N} X_{s_{n}}(t) H_{s_{n}}(t+1) \mid X(t)\right]=\sum_{l_{1}=1}^{N} X_{s_{l_{1}}}(t) U_{l_{1}}
$$

where

$$
\begin{aligned}
& U_{l_{1}}=\min \{K, R\} P\left(\mathcal{Z}_{l_{1}(N+1)}\right)+\min \left\{(K-R)^{+}, R\right\} \sum_{l_{2}>l_{1}} P\left(\mathcal{Z}_{l_{1} l_{2}}\right) P\left(\mathcal{Z}_{l_{2}(N+1)}\right)+\cdots \\
& \quad+\sum_{l_{k}>l_{k-1}>\cdots>l_{1}} \min \left\{(K-(k-1) R)^{+}, R\right\} P\left(\mathcal{Z}_{l_{1} l_{2}}\right) \cdots P\left(\mathcal{Z}_{l_{k-1} l_{k}}\right) P\left(\mathcal{Z}_{l_{k}(N+1)}\right)+\cdots \\
& \quad+\min \left\{(K-(N-1) R)^{+}, R\right\} P\left(\mathcal{Z}_{12}\right) \cdots P\left(\mathcal{Z}_{(N-1) N}\right) P\left(\mathcal{Z}_{N(N+1)}\right)
\end{aligned}
$$

Proof of this Claim is given in the following.

Proof of Claim 1: Consider the following decomposition of the connectivity process.

$$
\begin{aligned}
& D_{0}=\left\{C_{n}(t+1)=0, \text { for all } n \in \mathcal{N}\right\} \\
& D_{n}=\left\{C_{s_{n}}(t+1)=1, C_{s_{\ell}}(t+1)=0, \text { for } n<\ell \leq N\right\}
\end{aligned}
$$


Obviously $D_{0}=\mathcal{Z}_{0(N+1)}$ and $D_{n}=\mathcal{Z}_{n(N+1)}$. Therefore,

$$
\begin{array}{r}
E\left[\sum_{n=1}^{N} X_{s_{n}}(t) H_{s_{n}}(t+1) \mid X(t)\right]=\sum_{l_{1}=0}^{N} E\left[\sum_{n=1}^{N} X_{s_{n}}(t) H_{s_{n}}(t+1) \mid X(t), D_{l_{1}}\right] P\left(D_{l_{1}}\right) \\
=\sum_{l_{1}=1}^{N} E\left[\sum_{n=1}^{N} X_{s_{n}}(t) H_{s_{n}}(t+1) \mid X(t), \mathcal{Z}_{l_{1}(N+1)}\right] P\left(\mathcal{Z}_{l_{1}(N+1)}\right)
\end{array}
$$

The last equality is due to the fact that when the system is in condition $D_{0}$, there is no departure from the system. According to the LCQ policy rule, we have

$$
\begin{aligned}
& E\left[\sum_{n=1}^{N} X_{s_{n}}(t) H_{s_{n}}(t+1) \mid X(t), \mathcal{Z}_{l_{1}(N+1)}\right] \\
& \quad=X_{s_{l_{1}}}(t) \min \{K, R\}+E\left[\sum_{n=1, n \neq l_{1}}^{N} X_{s_{n}}(t) H_{s_{n}}(t+1) \mid X(t), \mathcal{Z}_{l_{1}(N+1)}\right] \\
& \quad=X_{s_{l_{1}}}(t) \min \{K, R\}+E\left[\sum_{n=1}^{l_{1}-1} X_{s_{n}}(t) H_{s_{n}}(t+1) \mid X(t), \mathcal{Z}_{l_{1}(N+1)}\right] \\
& \quad=X_{s_{l_{1}}}(t) \min \{K, R\}+\sum_{l_{2}=1}^{l_{1}-1} E\left[\sum_{n=1}^{l_{1}-1} X_{s_{n}}(t) H_{s_{n}}(t+1) \mid X(t), \mathcal{Z}_{l_{2} l_{1}}, \mathcal{Z}_{l_{1}(N+1)}\right] P\left(\mathcal{Z}_{l_{2} l_{1}}\right)
\end{aligned}
$$

where the last equality comes from conditioning on $\mathcal{Z}_{l_{2} l_{1}}$. Therefore, equation (B.14) can be written as

$$
\begin{aligned}
& E\left[\sum_{n=1}^{N} X_{s_{n}}(t) H_{s_{n}}(t+1) \mid X(t)\right]=\sum_{l_{1}=1}^{N} X_{s_{l_{1}}}(t) \min \{K, R\} P\left(\mathcal{Z}_{l_{1}(N+1)}\right) \\
& \quad+\sum_{l_{1}=1}^{N} \sum_{l_{2}=1}^{l_{1}-1} E\left[\sum_{n=1}^{l_{1}-1} X_{s_{n}}(t) H_{s_{n}}(t+1) \mid X(t), \mathcal{Z}_{l_{2} l_{1}}, \mathcal{Z}_{l_{1}(N+1)}\right] P\left(\mathcal{Z}_{l_{2} l_{1}}\right) P\left(\mathcal{Z}_{l_{1}(N+1)}\right)
\end{aligned}
$$


According to LCQ, we have

$$
\begin{aligned}
& E\left[\sum_{n=1}^{l_{1}-1} X_{s_{n}}(t) H_{s_{n}}(t+1) \mid X(t), \mathcal{Z}_{l_{2} l_{1}} \mathcal{Z}_{l_{1}(N+1)}\right] \\
& \quad=X_{s_{l_{2}}}(t) \min \left\{(K-R)^{+}, R\right\}+E\left[\sum_{n=1}^{l_{2}-1} X_{s_{n}}(t) H_{s_{n}}(t+1) \mid X(t), \mathcal{Z}_{l_{2} l_{1}} \mathcal{Z}_{l_{1}(N+1)}\right]
\end{aligned}
$$

From (B.16) and (B.17) we have

$$
\begin{aligned}
& E\left[\sum_{n=1}^{N} X_{s_{n}}(t) H_{s_{n}}(t+1) \mid X(t)\right]=\sum_{l_{1}=1}^{N} X_{s_{l_{1}}}(t) \min \{K, R\} P\left(\mathcal{Z}_{l_{1}(N+1)}\right) \\
& \quad+\sum_{l_{1}=1}^{N} \sum_{l_{2}=1}^{l_{1}-1} X_{s_{l_{2}}}(t) \min \left\{(K-R)^{+}, R\right\} P\left(\mathcal{Z}_{l_{2} l_{1}}\right) P\left(\mathcal{Z}_{l_{1}(N+1)}\right) \\
& \quad+E\left[\sum_{n=1}^{l_{2}-1} X_{s_{n}}(t) H_{s_{n}}(t+1) \mid X(t), \mathcal{Z}_{l_{2} l_{1}} \mathcal{Z}_{l_{1}(N+1)}\right] P\left(\mathcal{Z}_{l_{2} l_{1}}\right) P\left(\mathcal{Z}_{l_{1}(N+1)}\right)
\end{aligned}
$$


Therefore,

$$
\begin{aligned}
& E\left[\sum_{n=1}^{N} X_{s_{n}}(t) H_{s_{n}}(t+1) \mid X(t)\right]=\sum_{l_{1}=1}^{N} X_{s_{l_{1}}}(t) \min \{K, R\} P\left(\mathcal{Z}_{l_{1}(N+1)}\right) \\
& +\sum_{l_{1}=1}^{N} \sum_{l_{2}=1}^{l_{1}-1} X_{s_{l_{2}}}(t) \min \left\{(K-R)^{+}, R\right\} P\left(\mathcal{Z}_{l_{2} l_{1}}\right) P\left(\mathcal{Z}_{l_{1}(N+1)}\right) \\
& +\sum_{l_{1}=1}^{N} \sum_{l_{2}=1}^{l_{1}-1} \ldots \sum_{l_{k}=1}^{l_{k-1}-1} X_{s_{l_{k}}}(t) \min \left\{(K-(k-1) R)^{+}, R\right\} P\left(\mathcal{Z}_{l_{k} l_{k-1}}\right) \cdots P\left(\mathcal{Z}_{l_{2} l_{1}}\right) P\left(\mathcal{Z}_{l_{1}(N+1)}\right) \\
& +\sum_{1 \leq l_{N}<\ldots<l_{2}<l_{1} \leq N} X_{s_{l_{N}}}(t) \min \left\{(K-(N-1) R)^{+}, R\right\} P\left(\mathcal{Z}_{l_{N} l_{N-1}}\right) \cdots P\left(\mathcal{Z}_{l_{2} l_{1}}\right) P\left(\mathcal{Z}_{l_{1}(N+1)}\right) \\
& =\sum_{l_{1}=1}^{N} \min \{K, R\} X_{s_{l_{1}}}(t) P\left(\mathcal{Z}_{l_{1}(L+1)}\right) \\
& +\sum_{l_{2}=1}^{N} \sum_{l_{1}=l_{2}+1}^{N} \min \left\{(K-R)^{+}, R\right\} X_{s_{l_{2}}}(t) P\left(\mathcal{Z}_{l_{2} l_{1}}\right) P\left(\mathcal{Z}_{l_{1}(N+1)}\right) \\
& +\sum_{l_{k}=1}^{N} \ldots \sum_{l_{2}=l_{3}+1}^{N} \sum_{l_{1}=l_{2}+1}^{N} X_{s_{l_{k}}}(t) \min \left\{(K-(k-1) R)^{+}, R\right\} \times \\
& P\left(\mathcal{Z}_{l_{k} l_{k-1}}\right) P\left(\mathcal{Z}_{l_{k-1} l_{k-2}}\right) \cdots P\left(\mathcal{Z}_{l_{2} l_{1}}\right) P\left(\mathcal{Z}_{l_{1}(N+1)}\right) \\
& +\min \left\{(K-(N-1) R)^{+}, R\right\} X_{s_{1}}(t) P\left(\mathcal{Z}_{12}\right) P\left(\mathcal{Z}_{23}\right) \cdots P\left(\mathcal{Z}_{(N-1) N}\right) P\left(\mathcal{Z}_{N(N+1)}\right) \\
& =\sum_{l_{1}=1}^{N} X_{s_{l_{1}}}(t) U_{l_{1}}
\end{aligned}
$$


where $U_{l_{1}}$ is defined as

$$
\begin{aligned}
U_{l_{1}}=\min \{ & K, R\} P\left(\mathcal{Z}_{l_{1}(N+1)}\right)+\min \left\{(K-R)^{+}, R\right\} \sum_{l_{2}>l_{1}} P\left(\mathcal{Z}_{l_{1} l_{2}}\right) P\left(\mathcal{Z}_{l_{2}(N+1)}\right)+\cdots \\
& +\sum_{l_{k}>l_{k-1}>\cdots>l_{1}} \min \left\{(K-(k-1) R)^{+}, R\right\} P\left(\mathcal{Z}_{l_{1} l_{2}}\right) \cdots P\left(\mathcal{Z}_{l_{k-1} l_{k}}\right) P\left(\mathcal{Z}_{l_{k}(N+1)}\right)+\cdots \\
& +\min \left\{(K-(N-1) R)^{+}, R\right\} P\left(\mathcal{Z}_{12}\right) \cdots P\left(\mathcal{Z}_{(N-1) N}\right) P\left(\mathcal{Z}_{N(N+1)}\right)
\end{aligned}
$$

We define set $Q^{j}$ as $Q^{j}=\left\{Q_{s_{j}}, Q_{s_{j+1}}, \ldots, Q_{s_{N}}\right\}$.

Claim 2: Let us define $W_{j}=\sum_{n=j}^{N} U_{n}$. Then

$$
W_{j}=\sum_{i=1}^{\left|Q^{j}\right|} \min \left\{(K-(i-1) R)^{+}, R\right\} P\left(E_{i}^{Q^{j}}\right)
$$

Proof of this Claim is given in the following.

Proof of Claim 2: The proof is based on applying mathematical induction. In other words, we show the validity of (B.20) for $j=N$. Then we accept (B.20) is satisfied for $j$. Then, we show it is also valid for $j-1$. For $j=N$ we have

$$
\begin{aligned}
W_{N}=\min \{K, R\} P\left(\mathcal{Z}_{N(N+1)}\right)=\min \{K, R\} P\left(E_{1}^{Q^{N}}\right) \\
=\sum_{n=1}^{\left|Q^{N}\right|} \min \left\{(K-(n-1) R)^{+}, R\right\} P\left(E_{n}^{Q^{N}}\right)
\end{aligned}
$$

Now, we accept that the statement is true in this case. We consider $W_{j-1}$ as follows.

$$
\begin{aligned}
& W_{j-1}=W_{j}+U_{j-1}=\sum_{n=1}^{\left|Q^{j}\right|} \min \left\{(K-(n-1) R)^{+}, R\right\} P\left(E_{n}^{Q^{j}}\right) \\
& +\min \{K, R\} P\left(\mathcal{Z}_{(j-1)(N+1)}\right)+\min \left\{(K-R)^{+}, R\right\} \sum_{l_{2}>j-1} P\left(\mathcal{Z}_{(j-1) l_{2}}\right) P\left(\mathcal{Z}_{l_{2}(N+1)}\right)+\cdots \\
& \quad+\sum_{l_{k}>l_{k-1}>\cdots>j-1} \min \left\{(K-(k-1) R)^{+}, R\right\} P\left(\mathcal{Z}_{(j-1) l_{2}}\right) \cdots P\left(\mathcal{Z}_{l_{k-1} l_{k}}\right) P\left(\mathcal{Z}_{l_{k}(N+1)}\right)+\cdots \\
& \quad+\min \left\{(K-(N-1) R)^{+}, R\right\} P\left(\mathcal{Z}_{12}\right) \cdots P\left(\mathcal{Z}_{(N-1) N}\right) P\left(\mathcal{Z}_{N(N+1)}\right)
\end{aligned}
$$


Using the fact that

$$
\begin{gathered}
\min \{K, R\} P\left(\mathcal{Z}_{(j-1)(N+1)}\right)+\min \{K, R\} P\left(E_{1}^{Q^{j}}\right)=P\left(E_{1}^{Q^{j-1}}\right) \\
\min \left\{(K-R)^{+}, R\right\} \sum_{l_{2}>l_{1}} P\left(\mathcal{Z}_{(j-1) l_{2}}\right) P\left(\mathcal{Z}_{l_{2}(N+1)}\right)+\min \{K-R, R\} P\left(E_{2}^{Q^{j}}\right) \\
\quad=\min \{K-R, R\} P\left(E_{2}^{Q^{j-1}}\right) \\
\vdots \quad \min \left\{(K-(k-1) R)^{+}, R\right\} P\left(\mathcal{Z}_{(j-1) l_{2}}\right) \cdots P\left(\mathcal{Z}_{l_{k-1} l_{k}}\right) P\left(\mathcal{Z}_{l_{k}(N+1)}\right) \\
\sum_{l_{k}>l_{k-1}>\cdots>j-1} \\
+\min \left\{(K-(k-1) R)^{+}, R\right\} P\left(E_{k}^{Q^{j}}\right)=\min \left\{(K-(n-1) R)^{+}, R\right\} P\left(E_{k}^{Q^{j-1}}\right)
\end{gathered}
$$

the result follows.

According to Claims 1 and 2, we can rewrite the second term in (B.10) as follows.

$$
\begin{aligned}
E\left[\sum_{n=1}^{N} X_{n}(t) H_{n}(t+1) \mid X(t)\right] & =E\left[\sum_{n=1}^{N} X_{s_{n}}(t) H_{s_{n}}(t+1) \mid X(t)\right] \\
& =\sum_{j=2}^{N}\left(X_{s_{j}}(t)-X_{s_{j-1}}(t)\right) W_{j}+W_{1} X_{s_{1}}(t)
\end{aligned}
$$

Using (B.8)-(B.11) and (B.23), equation (B.8) can be written as

$$
\begin{aligned}
& E[L(X(t+1))-L(X(t)) \mid X(t))] \\
& \quad \leq N\left(A_{\text {max }}^{2}+R^{2}\right)+2 E\left[\sum_{n=1}^{N} X_{n}(t)\left(X_{n}(t+1)-X_{n}(t)\right) \mid X(t)\right] \\
& \quad=N\left(A_{m a x}^{2}+R^{2}\right)+2 \sum_{j=2}^{N}\left(X_{s_{j}}(t)-X_{s_{j-1}}(t)\right)\left(\sum_{l=j}^{N} E\left[A_{s_{l}}(t+1)\right]-W_{j}\right) \\
& \quad+2\left(\sum_{l=1}^{N} E\left[A_{s_{l}}(t+1)\right]-W_{1}\right) X_{s_{1}}(t) \\
& \quad \leq N\left(A_{\max }^{2}+R^{2}\right)+2 \sum_{j=2}^{N}\left(X_{s_{j}}(t)-X_{s_{j-1}}(t)\right) m+2 m X_{s_{1}}(t) \\
& \quad=N\left(A_{\max }^{2}+R^{2}\right)+2 m X_{s_{N}}(t)
\end{aligned}
$$


where

$$
m=\max _{Q \subseteq\{1,2, \ldots, N\}, t}\left\{\sum_{n \in Q} E\left[A_{n}(t)\right]-\sum_{i=1}^{|Q|} \min \left\{(K-(i-1) R)^{+}, R\right\} P\left(E_{i}^{Q}\right)\right\} .
$$

According to the condition (3.9), $m<0$. By considering the fact that $2 m X_{s_{N}}(t) \leq$ $\frac{2 m}{N} \sum_{n=1}^{N} X_{n}(t)$, we can bound (B.24) by

$$
E[L(X(t+1))-L(X(t)) \mid X(t))] \leq N\left(A_{\max }^{2}+R^{2}\right)+\frac{2 m}{N} \sum_{n=1}^{N} X_{n}(t)
$$

According to Lemma A.1 (see Appendix A), the system is strongly stable under LCQ policy and the average total occupancy is bounded by

$$
\underset{t \rightarrow \infty}{\limsup } \frac{1}{t} \sum_{\tau=0}^{t-1} \sum_{n=1}^{N} E\left[X_{n}(\tau)\right] \leq \frac{N^{2}\left(A_{\max }^{2}+R^{2}\right)}{-2 m}
$$

\section{B.4 Proof of Lemma 3.5}

Proof: For a stable system, equation (3.15) must be satisfied for any subset of queues $Q \subseteq \mathcal{N}$, i.e.,

$$
\lim _{t \rightarrow \infty} \frac{1}{t} \sum_{\tau=1}^{t} \sum_{n \in Q} E\left[A_{n}(\tau)\right]=\lim _{t \rightarrow \infty} \frac{1}{t} \sum_{\tau=1}^{t} \sum_{n \in Q} \sum_{k=1}^{K} E\left[H_{n, k}(\tau)\right]
$$

Define sets $B_{k}(\tau)$ as $B_{k}(\tau)=\left\{C_{\ell, k}(\tau), X_{\ell}(\tau-1), \ell \in Q\right\}$. For $B_{k}(\tau)$, three disjoint cases are possible.

1. $B_{k}^{1}(\tau)=\left\{C_{n, k}(\tau)=0, n \in Q\right\}$.

2. $B_{k}^{2}(\tau)=\left\{C_{n, k}(\tau)=0, n \in Q\right\}^{c} \cap\left\{X_{n}(\tau-1)=0, n \in Q\right\}$.

3. $B_{k}^{3}(\tau)=\left\{C_{n, k}(\tau)=0, n \in Q\right\}^{c} \cap\left\{X_{n}(\tau-1)=0, n \in Q\right\}^{c}$.

By conditioning each term in the right hand side summation in (B.28) to the event 
$B_{k}(\tau)$ we have

$$
\sum_{k=1}^{K} \sum_{n \in Q} E\left[H_{n, k}(\tau)\right]=\sum_{k=1}^{K} E_{B_{k}(\tau)}\left[E\left[\sum_{n \in Q} H_{n, k}(\tau) \mid B_{k}(\tau)\right]\right]
$$

Note that $E\left[\sum_{n \in Q} H_{n, k}(\tau) \mid B_{k}^{j}(\tau)\right]=0$ for $j=1,2$ and $E\left[\sum_{n \in Q} H_{n, k}(\tau) \mid B_{k}^{3}(\tau)\right] \leq$ 1. Therefore, the left hand side of (B.29) can be bounded by the following.

$$
\sum_{k=1}^{K} \sum_{n \in Q} E\left[H_{n, k}(\tau)\right] \leq \sum_{k=1}^{K}\left(1-P\left[B_{k}^{1}(\tau)\right]-P\left[B_{k}^{2}(\tau)\right]\right)
$$

Note that $P\left[B_{k}^{2}(\tau)\right] \geq 0$ and $P\left[B_{k}^{1}(\tau)\right]=\prod_{n \in Q}\left(1-p_{n, k}\right)$. Finally, from (B.28) and (B.30) we conclude

$$
\lim _{t \rightarrow \infty} \frac{1}{t} \sum_{\tau=1}^{t} \sum_{n \in Q} E\left[A_{n}(\tau)\right] \leq \sum_{k=1}^{K}\left(1-\prod_{n \in Q}\left(1-p_{n, k}\right)\right)
$$

and the lemma follows.

\section{B.5 Proof of Lemma 3.6}

Proof: We will start with the Lyapunov drift evaluation and use the quadratic function $L(X(t))=\sum_{n=1}^{N} X_{n}^{2}(t)$ as our Lyapunov function. The Lyapunov drift for two successive time slots has the following form.

$$
\begin{aligned}
& E[L(X(t+1))-L(X(t)) \mid X(t))]=E\left[\sum_{n=1}^{N}\left(X_{n}^{2}(t+1)-X_{n}^{2}(t)\right) \mid X(t)\right] \\
& \quad=E\left[\sum_{n=1}^{N}\left(X_{n}(t+1)-X_{n}(t)\right)^{2} \mid X(t)\right]+2 E\left[\sum_{n=1}^{N} X_{n}(t)\left(X_{n}(t+1)-X_{n}(t)\right) \mid X(t)\right]
\end{aligned}
$$


For the the first term we have the following bound.

$$
\begin{array}{r}
E\left[\sum_{n=1}^{N}\left(X_{n}(t+1)-X_{n}(t)\right)^{2} \mid X(t)\right]=E\left[\sum_{n=1}^{N}\left(A_{n}(t+1)-\sum_{k=1}^{K} H_{n, k}(t)\right)^{2} \mid X(t)\right] \\
\leq E\left[\sum_{n=1}^{N} A_{n}^{2}(t+1) \mid X(t)\right]+E\left[\sum_{n=1}^{N}\left(\sum_{k=1}^{K} H_{n, k}(t)\right)^{2} \mid X(t)\right] \leq N A_{\max }^{2}+K^{2}
\end{array}
$$

The last inequality holds since $\sum_{k=1}^{K} H_{n, k}(t) \geq 0$ and thus $\sum_{n=1}^{N}\left(\sum_{k=1}^{K} H_{n, k}(t)\right)^{2} \leq$ $\left(\sum_{n=1}^{N} \sum_{k=1}^{K} H_{n, k}(t)\right)^{2} \leq K^{2}$. Now assume that we select the servers for service according to an arbitrary order $s_{1}, s_{2}, \ldots, s_{K}$. Thus, for the second term in (B.32) we have

$$
\begin{aligned}
& E\left[\sum_{n=1}^{N} X_{n}(t)\left(X_{n}(t+1)-X_{n}(t)\right) \mid X(t)\right] \\
& \quad=E\left[\sum_{n=1}^{N} X_{n}(t)\left(A_{n}(t+1)-\sum_{k=1}^{K} H_{n, k}(t+1)\right) \mid X(t)\right] \\
& \quad=E\left[\sum_{n=1}^{N} X_{n}(t) A_{n}(t+1) \mid X(t)\right]-\sum_{k=1}^{K} E\left[\sum_{n=1}^{N} X_{n}(t) H_{n, s_{k}}(t+1) \mid X(t)\right]
\end{aligned}
$$

The first term in (B.34) can be written as follows.

$$
E\left[\sum_{n=1}^{N} X_{n}(t) A_{n}(t+1) \mid X(t)\right]=\sum_{n=1}^{N} E\left[A_{n}(t+1)\right] X_{n}(t)
$$

Now, we introduce the following notation. We sort the queue lengths at time slot $t$ in an ascending order $X_{q_{1}}, X_{q_{2}}, \ldots, X_{q_{N}}$, i.e., $X_{q_{n}}(t) \geq X_{q_{n-1}}(t)$ for all $i=2, \ldots, N$, and if $X_{q_{n}}(t)=X_{q_{n-1}}(t)$, then $q_{n} \geq q_{n-1}$. Furthermore, consider the following decomposition of the channel processes for each server $k$.

$$
\begin{aligned}
& D_{0}^{k}=\left\{C_{n, k}(t+1)=0, \text { for all } n \in \mathcal{N}\right\} \\
& D_{n}^{k}=\left\{C_{q_{n}, k}(t+1)=1, C_{q_{\ell}, k}(t+1)=0, n<\ell \leq N\right\}
\end{aligned}
$$


The probability of events $D_{n}^{k}$ is given by

$$
P\left(D_{0}^{k}\right)=\prod_{n=1}^{N}\left(1-p_{n, k}\right) \quad, \quad P\left(D_{n}^{k}\right)=p_{q_{n}, k} \prod_{u=n+1}^{N}\left(1-p_{q_{u}, k}\right)
$$

Therefore,

$$
\begin{aligned}
E\left[\sum_{n=1}^{N} X_{n}(t) H_{n, s_{k}}(t+1) \mid X(t)\right] & =E\left[\sum_{n=1}^{N} X_{q_{n}}(t) H_{q_{n}, s_{k}}(t+1) \mid X(t)\right] \\
= & \sum_{l=0}^{N} E\left[\sum_{n=1}^{N} X_{q_{n}}(t) H_{q_{n}, s_{k}}(t+1) \mid X(t), D_{l}^{s_{k}}\right] P\left(D_{l}^{s_{k}}\right)
\end{aligned}
$$

Note that

$$
E\left[\sum_{n=1}^{N} X_{q_{n}}(t) H_{q_{n}, s_{k}}(t+1) \mid X(t), D_{l}^{s_{k}}\right] \geq\left(X_{q_{l}}(t)-(k-1)\right)^{+}
$$

Therefore, equation (B.36) can be bounded by

$$
\begin{aligned}
E[ & {\left[\sum_{n=1}^{N} X_{n}(t) H_{n, s_{k}}(t+1) \mid X(t)\right] \geq \sum_{l=1}^{N}\left(X_{q_{l}}(t)-(k-1)\right) p_{q_{l}, s_{k}} \prod_{j=l+1}^{N}\left(1-p_{q_{j}, s_{k}}\right) } \\
= & \sum_{l=1}^{N} X_{q_{l}}(t) p_{q_{l}, s_{k}} \prod_{j=l+1}^{N}\left(1-p_{q_{j}, s_{k}}\right)-\sum_{l=1}^{N}(k-1) p_{q_{l}, s_{k}} \prod_{j=l+1}^{N}\left(1-p_{q_{j}, s_{k}}\right) \\
= & \sum_{j=2}^{N}\left(X_{q_{j}}(t)-X_{q_{j-1}}(t)\right)\left(1-\prod_{l=j}^{N}\left(1-p_{q_{l}, s_{k}}\right)\right)+X_{q_{1}}(t)\left(1-\prod_{j=1}^{N}\left(1-p_{q_{j}, s_{k}}\right)\right) \\
& \quad-(k-1)\left(1-\prod_{j=1}^{N}\left(1-p_{q_{j}, s_{k}}\right)\right)
\end{aligned}
$$


Equation (B.35) also can be written as follows.

$$
\begin{aligned}
& \sum_{n=1}^{N} E\left[A_{n}(t+1)\right] X_{n}(t)=\sum_{l=1}^{N} E\left[A_{q_{l}}(t+1)\right] X_{q_{l}}(t) \\
& \quad=\sum_{j=2}^{N}\left(X_{q_{j}}(t)-X_{q_{j-1}}(t)\right) \sum_{l=j}^{N} E\left[A_{q_{l}}(t+1)\right]+X_{q_{1}}(t) \sum_{l=1}^{N} E\left[A_{q_{l}}(t+1)\right]
\end{aligned}
$$

Note that the last term in (B.37) can be bounded by $k-1$. Using equations (B.34)(B.38) and the fact that $\sum_{k=1}^{K}(k-1)=\frac{K(K-1)}{2}$ we have the following bound for the second term in (B.32).

$$
\begin{aligned}
E[ & \left.\sum_{n=1}^{N} X_{n}(t)\left(X_{n}(t+1)-X_{n}(t)\right) \mid X(t)\right] \\
& \leq \sum_{j=2}^{N}\left(X_{q_{j}}(t)-X_{q_{j-1}}(t)\right) \cdot\left(\sum_{l=j}^{N} E\left[A_{q_{l}}(t+1)\right]-\sum_{k=1}^{K}\left(1-\prod_{l=j}^{N}\left(1-p_{q_{l}, k}\right)\right)\right) \\
& +X_{q_{1}}(t) \cdot\left(\sum_{l=1}^{N} E\left[A_{q_{l}}(t+1)\right]-\sum_{k=1}^{K}\left(1-\prod_{j=1}^{N}\left(1-p_{q_{j}, k}\right)\right)\right)+\frac{K(K-1)}{2}
\end{aligned}
$$

We define $m=\max _{Q \subseteq\{1,2, \ldots, N\}, t}\left\{\sum_{n \in Q} E\left[A_{n}(t)\right]-K+\sum_{k=1}^{K} \prod_{n \in Q}\left(1-p_{n, k}\right)\right\}$. Therefore, equation (B.39) can be bounded by the following.

$$
\begin{aligned}
& E\left[\sum_{n=1}^{N} X_{n}(t)\left(X_{n}(t+1)-X_{n}(t)\right) \mid X(t)\right] \\
& \quad \leq \sum_{j=2}^{N}\left(X_{q_{j}}(t)-X_{q_{j-1}}(t)\right) m+X_{q_{1}}(t) m+\frac{K(K-1)}{2}=X_{q_{N}}(t) m+\frac{K(K-1)}{2}
\end{aligned}
$$

Putting all together and according to (B.32), (B.33) and (B.40), the Lyapunov drift in equation (B.32) is upper bounded by the following.

$$
E[L(X(t+1))-L(X(t)) \mid X(t))] \leq N A_{\max }^{2}+2 K^{2}-\epsilon\left(N X_{q_{N}}(t)\right)-K
$$

where $\epsilon=-\frac{2 m}{N}$. Note that $m$ is negative and therefore $\epsilon>0$. Consequently, the 
Lyapunov drift is bounded by

$$
E[L(X(t+1))-L(X(t)) \mid X(t))] \leq N A_{\max }^{2}+2 K^{2}-K-\epsilon \sum_{n=1}^{N} X_{n}(t)
$$

According to Lemma A.1, the multi-queue multi-server system with random connectivities is stable under Maximum Weight policy as long as condition (3.19) is satisfied and also the time average expected congestion in the system is bounded by

$$
\limsup _{t \rightarrow \infty} \frac{1}{t} \sum_{\tau=0}^{t-1} \sum_{n=1}^{N} E\left[X_{n}(\tau)\right] \leq \frac{N A_{\max }^{2}+2 K^{2}-K}{\epsilon}
$$

\section{B.6 Proof of Lemma 3.7}

Proof: If we write equation (3.27) for each queue $n$ and for $\tau=1,2, \ldots, t$ and then add them up, we will have

$$
X_{n}(t)=X_{n}(0)-\sum_{\tau=1}^{t} \sum_{k=1}^{K} H_{n, k}(\tau)+\sum_{\tau=1}^{t} A_{n}(\tau)
$$

By multiplying the vector $\alpha$ by the queue length vector $X(t)$ we have

$$
\alpha X^{\mathrm{T}}(t)=\alpha X^{\mathrm{T}}(0)-\sum_{\tau=1}^{t} \alpha H(\tau) \underline{1}_{K}^{\mathrm{T}}+\sum_{\tau=1}^{t} \alpha A^{\mathrm{T}}(\tau)
$$

Taking the expectation of both sides, dividing by $t$ and then taking the limit as $t$ goes to infinity, we will have the following.

$$
\begin{aligned}
\lim _{t \rightarrow \infty} & \frac{1}{t} \alpha E[X(t)]^{\mathrm{T}} \\
& =\lim _{t \rightarrow \infty} \frac{1}{t} \alpha E[X(0)]^{\mathrm{T}}-\lim _{t \rightarrow \infty} \frac{1}{t} \sum_{\tau=1}^{t} \alpha E[H(\tau)] \underline{1}_{K}^{\mathrm{T}}+\lim _{t \rightarrow \infty} \frac{1}{t} \sum_{\tau=1}^{t} \alpha E[A(\tau)]^{\mathrm{T}}
\end{aligned}
$$

According to Lemma 3.3 in $[1]$ and the assumption that $E[X(0)]<\infty$, the left hand side term and the first term in the right hand side term are equal to zero and the lemma follows. 


\section{B.7 Proof of Lemma 3.8}

Proof: Recall that $I^{(\pi)}(t)$ denotes the employed matching by policy $\pi$ at time slot $t$. In (3.28), by conditioning the process $H(t)$ on the connectivity process, we will have

$$
\alpha \lambda^{\mathrm{T}}=\lim _{t \rightarrow \infty} \frac{1}{t} \sum_{\tau=1}^{t} \alpha E[H(\tau)] \underline{1}_{K}^{\mathrm{T}}=\lim _{t \rightarrow \infty} \frac{1}{t} \sum_{\tau=1}^{t} \sum_{c \in \mathcal{C}} P(c) \alpha E[H(\tau) \mid C(\tau)=c] \underline{1}_{K}^{\mathrm{T}}
$$

For an arbitrary vector $v$ of size $|v|,(v)^{+}$is defined as a vector of the same size whose $i$ 'th element, $(v)_{i}^{+}$, is

$$
(v)_{i}^{+}= \begin{cases}0, & \text { if } v_{i}<0 \\ v_{i}, & \text { if } v_{i} \geq 0\end{cases}
$$

Therefore, (B.46) can be bounded by

$$
\begin{aligned}
\alpha \lambda^{\mathrm{T}} & \leq \lim _{t \rightarrow \infty} \frac{1}{t} \sum_{\tau=1}^{t} \sum_{c \in \mathcal{C}} P(c)(\alpha)^{+} E\left[c \circledast I^{(\pi)}(\tau)\right] \underline{1}_{K}^{\mathrm{T}} \\
& \leq \lim _{t \rightarrow \infty} \frac{1}{t} \sum_{\tau=1}^{t} \sum_{c \in \mathcal{C}} P(c) \max _{M \in \mathcal{I}}\left((\alpha)^{+}(c \circledast I) \underline{1}_{K}^{\mathrm{T}}\right) \\
& =\sum_{c \in \mathcal{C}} P(c) \max _{M \in \mathcal{I}}\left((\alpha)^{+}(c \circledast I) \underline{1}_{K}^{\mathrm{T}}\right) .
\end{aligned}
$$

Now, consider two different cases.

- $\alpha \in \mathbb{R}_{+}^{N}$ : The result follows directly from (B.48) since $(\alpha)^{+}=\alpha$.

- $\alpha \notin \mathbb{R}_{+}^{N}$ : In this case, from (B.46) we have the following inequality.

$$
\alpha \lambda^{\mathrm{T}} \leq \sum_{c \in \mathcal{C}} P(c) \max _{M \in \mathcal{I}}\left((\alpha)^{+}(c \circledast I) \underline{1}_{K}^{\mathrm{T}}\right)
$$

However, $(\alpha)^{+} \in \mathbb{R}_{+}^{N}$ and according to the previous case we also have

$$
(\alpha)^{+} \lambda^{\mathbf{T}} \leq \sum_{c \in \mathcal{C}} P(c) \max _{I \in \mathcal{I}}\left((\alpha)^{+}(c \circledast I) \underline{1}_{K}^{\mathrm{T}}\right)
$$

Noting (B.50) and the fact that $\lambda \in \mathbb{R}_{+}^{N}$, we conclude that (B.49) is redundant. 


\section{B.8 Proof of Lemma 3.9}

Proof: Let $\mathcal{I}^{\alpha}=\left\{I^{\alpha}(c), c \in \mathcal{C}\right\}$ denote a set of matchings that solve (3.30). $\mathcal{I}^{\alpha}$ is not a unique solution of (3.30) and there may be more than one set of matchings $\mathcal{I}^{\alpha}$ whose elements maximize (3.30). Let $\mathbb{I}^{\alpha}=\left\{\mathcal{I}_{i}^{\alpha}, 1 \leq i \leq\left|\mathbb{I}^{\alpha}\right|\right\}$ denote the set of all distinguished solutions of (3.30). Obviously $\left|\mathbb{I}^{\alpha}\right|<\infty$, since set $\mathcal{I}$ is finite. Note that each solution $\mathcal{I}_{i}^{\alpha}=\left\{I_{i}^{\alpha}(c), c \in \mathcal{C}\right\}, 1 \leq i \leq\left|\mathbb{I}^{\alpha}\right|$ is corresponding to a deterministic policy with the average service rate vector

$$
R_{i}^{\alpha}=\left(\sum_{c \in \mathcal{C}} P(c)\left(c \circledast I_{i}^{\alpha}(c)\right) \underline{1}_{K}^{\mathrm{T}}\right)^{\mathrm{T}}
$$

and therefore, according to (3.24) each solutions of (3.30) is associated with a vertex of the polytope $\mathcal{R}$. Since there are $\left|\mathbb{I}^{\alpha}\right|$ distinguished solution of $(3.30),\left|\mathbb{I}^{\alpha}\right|$ vertices of polytope $\mathcal{R}$ are on the hyperplane associated with (3.29). In other words, each face of $\mathcal{R}$ associated to (3.29) is represented by $F_{\alpha}=$ conv.hull $l_{1 \leq i \leq\left|\mathbb{I}^{\alpha}\right|} R_{i}^{\alpha}$.

We now introduce the following mapping.

$$
\begin{gathered}
\Omega: \mathbb{R}_{+}^{N} \longmapsto\{0,1\}^{N} \\
\alpha=\left(\alpha_{1}, \alpha_{2}, \ldots, \alpha_{N}\right) \longmapsto \beta=\left(\beta_{1}, \beta_{2}, \ldots, \beta_{N}\right): \beta_{n}=\left\{\begin{array}{ll}
0 & \text { if } \alpha_{n}=0 \\
1 & \text { if } \alpha_{n}>0
\end{array} \quad \forall n \in \mathcal{N}\right.
\end{gathered}
$$

Mapping $\Omega$ takes any vector $\alpha \in \mathbb{R}_{+}^{N}$ and returns another $N$ dimensional vector $\beta$ whose elements are in $\{0,1\}$. For each element $\beta_{n}, \Omega$ returns " 0 " if $\alpha_{n}$ is zero and returns "1" if $\alpha_{n}$ is non-zero. In the following, our objective is to prove that $\mathbb{I}^{\alpha} \subseteq \mathbb{I}^{\Omega(\alpha)}$ (or equivalently $\left.F_{\alpha} \subseteq F_{\Omega(\alpha)}\right)$. To this end, we have to show that $\mathcal{I}_{i}^{\alpha} \in \mathbb{I}^{\Omega(\alpha)}$ for all $1 \leq i \leq\left|\mathbb{I}^{\alpha}\right|$. Remember that $\mathcal{I}_{i}^{\alpha}=\left\{I_{i}^{\alpha}(c), c \in \mathcal{C}\right\}$ and $I_{i}^{\alpha}(c)=\arg \max _{I \in \mathcal{I}} \alpha(c \circledast I) \underline{1}_{K}^{\mathrm{T}}$.

We claim that any matching $I^{\alpha}(c)$ that maximizes $\alpha(c \circledast I) \underline{1}_{K}^{\mathrm{T}}$ also maximizes $\Omega(\alpha)(c \circledast I) \underline{1}_{K}^{\mathrm{T}}$. Note that if a matching $I^{\alpha}(c)$ maximizes $\alpha(c \circledast I) \underline{1}_{K}^{\mathrm{T}}$, this means that $I^{\alpha}(c)$ is a maximum matching in a bipartite graph $G^{\alpha}=\left(\mathcal{N}, \mathcal{K}, \mathcal{E}^{\alpha}\right)$ (see Figure B.1). In $G^{\alpha}, \mathcal{N}$ and $\mathcal{K}$ are the two sets of vertices in each part of the graph and $\mathcal{E}^{\alpha}=\left\{e_{n, k}^{\alpha}, \forall n \in \mathcal{N}, \forall k \in \mathcal{K}\right\}$ is the set of edges between these two parts where $e_{n, k}^{\alpha}=$ 


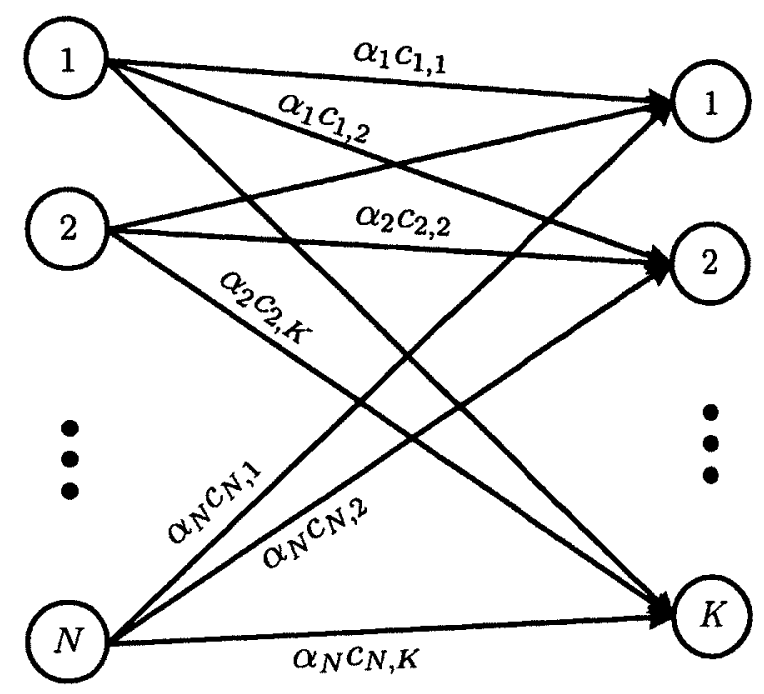

Figure B.1: Graph $G^{\alpha}$

$\alpha_{n} c_{n, k}$. If there exists $I^{\alpha}(c)$ which maximizes $\alpha(c \circledast I) \underline{1}_{K}^{\mathrm{T}}$ but not $\Omega(\alpha)(c \circledast I) \underline{1}_{K}^{\mathrm{T}}$ this means that matching $I^{\alpha}(c)$ is a maximum matching for graph $G^{\alpha}$ but not for $G^{\Omega(\alpha)}$. According to Lemma 5.2 (in Chapter 5) there must exist a server reallocation for matching $I^{\alpha}(c)$ in graph $G^{\Omega(\alpha)}$ that results into another matching $I^{\prime}$ whose matching weight is greater than that of $I^{\alpha}(c)$ Since $\Omega(\alpha) \in\{0,1\}^{N}$, this translates to the fact that there is at least one queue $m$ with $\alpha_{m}>0$ which is not receiving service under $I^{\alpha}(c)$ but is serviced under $I^{\prime}$ while the service of other queues is not disturbed. If we apply matching $I^{\prime}$ to graph $G^{\alpha}$ we have the same results, i.e., there is at least one queue $m$ which is not receiving service under $I^{\alpha}(c)$ but it is serviced under $I^{\prime}$ while the service of other queues is not disturbed. This means that matching weight of $I^{\alpha}(c)$ is less than that of matching $I^{\prime}$, i.e., matching $I^{\alpha}(c)$ is not maximum matching in graph $G^{\alpha}$. This contradicts our assumption that $I^{\alpha}(c)$ is maximum matching in graph $G^{\alpha}$.

Therefore, for all $c \in \mathcal{C}$, all the maximum matchings $I^{\alpha}(c)$ in graph $G^{\alpha}$ are also maximum matchings in graph $G^{\Omega(\alpha)}$. In other words, all the solutions of "arg $\max \alpha(c \circledast I) \underline{1}_{K}^{\mathrm{T}}$ " are solutions for " $\arg \max \Omega(\alpha)(c \circledast I) \underline{1}_{K}^{\mathrm{T}}$ ". Hence, $\mathcal{I}_{i}^{\alpha} \in \mathbb{I}^{\Omega(\alpha)}$ and therefore $\mathbb{I}^{\alpha} \subseteq \mathbb{I}^{\Omega(\alpha)}$. Consequently $F_{\alpha} \subseteq F_{\Omega(\alpha)}$.

Thus, according to Lemma 3.8 and the fact that $F_{\alpha} \subseteq F_{\Omega(\alpha)}$, we conclude that all the hyperplanes associated to half spaces (3.29) with $\alpha \in \mathbb{R}_{+}^{N}-\{0,1\}^{N}$ are redundant in describing polytope $\mathcal{R}$. Note that vector $\alpha=\underline{0}_{N}$ will result in the obvious equality $0=0$ and therefore is redundant too. Consequently, from all the vectors $\alpha \in \mathbb{R}_{+}^{N}$, just 
$\alpha \in\{0,1\}^{N}-\left\{\underline{0}_{N}\right\}$ are sufficient to describe polytope $\mathcal{R}$ and the theorem follows.

\section{B.9 Proof of Lemma 3.10}

Proof: We start with the Lyapunov function evaluation. We use the quadratic function $L(X)=\sum_{n=1}^{N} X_{n}^{2}(t)$ as our Lyapunov function. The Lyapunov drift $D$ for two successive time slots has the following form.

$$
\begin{aligned}
& D(t+1)=E[L(X(t+1))-L(X(t)) \mid X(t)] \\
& \quad=E\left[\sum_{n=1}^{N}\left(X_{n}^{2}(t+1)-X_{n}^{2}(t)\right) \mid X(t)\right] \\
& =E\left[\sum_{n=1}^{N}\left(X_{n}(t+1)-X_{n}(t)\right)^{2} \mid X(t)\right] \\
& \quad+2 E\left[\sum_{n=1}^{N} X_{n}(t)\left(X_{n}(t+1)-X_{n}(t)\right) \mid X(t)\right]
\end{aligned}
$$

For the the first term, we have

$$
\begin{aligned}
E\left[\sum_{n=1}^{N}\left(X_{n}(t+1)-X_{n}(t)\right)^{2} \mid X(t)\right] \\
\quad=E\left[\sum_{n=1}^{N}\left(A_{n}(t+1)-\sum_{k=1}^{K} H_{n, k}(t+1)\right)^{2} \mid X(t)\right] \\
=E\left[\sum_{n=1}^{N} A_{n}^{2}(t+1) \mid X(t)\right] \\
\quad-2 E\left[\sum_{n=1}^{N} \sum_{k=1}^{K} A_{n}(t+1) H_{n, k}(t+1) \mid X(t)\right] \\
\left.\quad+E\left[\sum_{n=1}^{N}\left(\sum_{k=1}^{K} H_{n, k}(t+1)\right)^{2} \mid X(t)\right]^{2}\right] \\
\quad \leq N A_{\max }^{2}+\sum_{n=1}^{N} E\left[\left(\sum_{k=1}^{K} H_{n, k}(t+1)\right)^{2} \mid X(t)\right] .
\end{aligned}
$$


For the second term in (B.53), we have

$$
\begin{aligned}
E\left[\sum_{n=1}^{N} X_{n}(t)\left(X_{n}(t+1)-X_{n}(t)\right) \mid X(t)\right] \\
\quad=E\left[\sum_{n=1}^{N} X_{n}(t)\left(A_{n}(t+1)-\sum_{k=1}^{K} H_{n, k}(t+1)\right) \mid X(t)\right] \\
\quad=\sum_{n=1}^{N} X_{n}(t) E\left[A_{n}(t+1)\right]-\sum_{n=1}^{N} E\left[X_{n}(t) \sum_{k=1}^{K} H_{n, k}(t+1) \mid X(t)\right] .
\end{aligned}
$$

Therefore, Lyapunov drift $D(t+1)$ can be written as

$$
\begin{aligned}
& D(t+1) \leq N A_{\max }^{2}+\sum_{n=1}^{N} E\left[\left(\sum_{k=1}^{K} H_{n, k}(t+1)\right)^{2} \mid X(t)\right] \\
& \quad+2 \sum_{n=1}^{N} X_{n}(t) E\left[A_{n}(t+1)\right]-2 \sum_{n=1}^{N} E\left[X_{n}(t) \sum_{k=1}^{K} H_{n, k}(t+1) \mid X(t)\right] .
\end{aligned}
$$

In the following, we show that the Lyapunov drift in (B.56) is bounded as follows.

$$
\begin{aligned}
& D(t+1) \leq N A_{\text {max }}^{2}+\sum_{n=1}^{N} E\left[\left(\sum_{k=1}^{K} C_{n, k}(t+1) I_{n, k}(t+1)\right)^{2} \mid X(t)\right] \\
& \quad+2 \sum_{n=1}^{N} X_{n}(t) E\left[A_{n}(t+1)\right]-2 \sum_{n=1}^{N} E\left[X_{n}(t) \sum_{k=1}^{K} C_{n, k}(t+1) I_{n, k}(t+1) \mid X(t)\right]
\end{aligned}
$$

where $C(t+1)=\left(C_{n, k}(t+1)\right), n \in \mathcal{N}, k \in \mathcal{K}$ is the observed connectivity matrix at the beginning of time slot $t+1$. To prove (B.57), note that for each queue $n \in \mathcal{N}$ one of the following conditions is satisfied.

- $\sum_{k=1}^{K} H_{n, k}(t+1)=\sum_{k=1}^{K} C_{n, k}(t+1) I_{n, k}(t+1)$ : In this case, we can easily check 
that

$$
\begin{aligned}
& E\left[X_{n}^{2}(t+1)-X_{n}^{2}(t) \mid X(t)\right] \leq N A_{\max }^{2} \\
& \quad+E\left[\left(\sum_{k=1}^{K} C_{n, k}(t+1) I_{n, k}(t+1)\right)^{2} \mid X(t)\right] \\
& \quad+2 X_{n}(t) E\left[A_{n}(t+1)\right]-2 E\left[X_{n}(t) \sum_{k=1}^{K} C_{n, k}(t+1) I_{n, k}(t+1) \mid X(t)\right]
\end{aligned}
$$

- $\sum_{k=1}^{K} H_{n, k}(t+1)<\sum_{k=1}^{K} C_{n, k}(t+1) I_{n, k}(t+1)$ : In this case, $\sum_{k=1}^{K} H_{n, k}(t+1)=$ $X_{n}(t)$ and $X_{n}(t+1)=A_{n}(t+1)$ as there are not enough packets in queue $n$ to be served at time slot $t+1$. It is not hard to check that inequality (B.58) is also satisfied in this case.

According to the above discussion, we can observe that inequality (B.58) is satisfied for all the queues and therefore (B.57) follows.

Using the the fact that $\sum_{k=1}^{K} C_{n, k}(t+1) I_{n, k}(t+1) \geq 0$, we have the following inequality.

$$
\begin{aligned}
& \sum_{n=1}^{N}\left(\sum_{k=1}^{K} C_{n, k}(t+1) I_{n, k}(t+1)\right)^{2} \\
& \quad \leq\left(\sum_{n=1}^{N} \sum_{k=1}^{K} C_{n, k}(t+1) I_{n, k}(t+1)\right)^{2} \leq(\min \{N, K\})^{2}
\end{aligned}
$$

Hence, the Lyapunov drift (B.57) is bounded by

$$
\begin{array}{r}
D(t+1) \leq N A_{\max }^{2}+(\min \{N, K\})^{2}+2 \sum_{n=1}^{N} X_{n}(t) E\left[A_{n}(t+1)\right] \\
-2 \sum_{n=1}^{N} E\left[X_{n}(t) \sum_{k=1}^{K} C_{n, k}(t+1) I_{n, k}(t+1) \mid X(t)\right]
\end{array}
$$

By conditioning the last term of (B.60) on the connectivity process at time slot $t+1$, 
we will have

$$
\begin{aligned}
& D(t+1) \leq N A_{\max }^{2}+(\min \{N, K\})^{2} \\
& \quad+2 E\left[X(t)\left(A^{\mathbf{T}}(t+1)-\sum_{c \in \mathcal{C}} P(c)(c \circledast M(t+1)) \underline{\mathbf{1}}_{K}^{\mathbf{T}}\right) \mid X(t)\right] .
\end{aligned}
$$

Note that matching $I$ in (B.61) depends on the selected policy. According to (3.34), we can see that by selecting MWM policy, the second term of (B.61) will be minimized and therefore the right hand side term in (B.61) will be minimized over all the existing server assignment policies. In other words, for MWM policy and any arbitrary server assignment policy $\pi$ we have

$$
\begin{aligned}
& D^{\mathrm{MWM}}(t+1) \leq N A_{\max }^{2}+(\min \{N, K\})^{2} \\
& \quad+2 E\left[X(t)\left(A^{\mathrm{T}}(t+1)-\sum_{c \in \mathcal{C}} P(c)\left(c \circledast I^{(\mathrm{MWM})}(t+1)\right) \underline{1}_{K}^{\mathrm{T}}\right) \mid X(t)\right] \\
& \leq N A_{\max }^{2}+(\min \{N, K\})^{2} \\
& \quad+2 E\left[X(t)\left(A^{\mathrm{T}}(t+1)-\sum_{c \in \mathcal{C}} P(c)\left(c \circledast I^{(\pi)}(t+1)\right) \underline{1}_{K}^{\mathrm{T}}\right) \mid X(t)\right] .
\end{aligned}
$$

where $D^{\mathrm{MWM}}(t+1)$ is the Lyapunov drift of MWM policy at time slot $t+1$.

It is important to note that the set of inequalities in (3.35) determines an open polytope $\mathcal{R}^{\prime}$ for which we have $\mathcal{R}^{\prime}=\mathcal{R}$-bound $(\mathcal{R})$. In fact, Lemma 3.10 states that if the vector $\lambda$ is strictly inside polytope $\mathcal{R}$ then the system is stable. Now, consider the system with arrival processes for which we have $\lambda \in \mathcal{R}^{\prime}$ at each time slot $t$. Suppose that $\delta$ is a positive real number such that $\lambda+\delta \underline{1}_{N} \in$ bound $(\mathcal{R})$. According to our discussion about deterministic and randomized policies, there exists a randomized policy $I^{r n d}=\left\{I^{r n d}(t)\right\}_{t=1}^{\infty}$ for which we will have

$$
E\left[\left(\sum_{c \in \mathcal{C}} P(c)\left(c \circledast I^{r n d}(t)\right) \underline{1}_{K}^{\mathrm{T}}\right)^{\mathrm{T}}\right]=\lambda+\delta \underline{1}_{N} .
$$


Therefore,

$$
E\left[X(t)\left(A^{\mathrm{T}}(t+1)-\sum_{c \in \mathcal{C}} P(c)\left(c \circledast I^{(r n d)}(t+1)\right) \underline{1}_{K}^{\mathrm{T}}\right) \mid X(t)\right]=-\delta \sum_{n=1}^{N} X_{n}(t) .
$$

Putting together from (B.61) to (B.64), we conclude that

$$
D^{\mathrm{MWM}}(t+1) \leq N A_{m a x}^{2}+(\min \{N, K\})^{2}-2 \delta \sum_{n=1}^{N} X_{n}(t)
$$

and according to Lemma 4.1 in [1], this proves the stability of system. Furthermore, according to the definition of $\delta$ and (3.35), we conclude that $\delta=\min _{t, \alpha \in\{0,1\}^{N}-\left\{\underline{0}_{N}\right\}}\left\{\frac{1}{\sum_{i=1}^{N} \alpha_{i}}\left(\sum_{c \in \mathcal{C}} P(c) \max _{I \in \mathcal{I}}\left(\alpha(c \circledast I) \underline{1}_{K}^{\mathrm{T}}\right)-\alpha E[A(t)]^{\mathrm{T}}\right)\right\}$. Therefore, the upper bound given in (3.36) for the average total queue occupancy of MWM policy holds. 


\section{Appendix $\mathrm{C}$}

\section{Proofs of the Results of Chapter 4}

\section{C.1 Proof of Lemma 4.5}

Proof: As we explained before, $\mathcal{I}^{\alpha}$ denotes a set of allocation matrices of $\left\{I_{s}^{\alpha}, s \in \mathcal{S}\right\}$ that maximize the right hand side of (4.10) and we discussed that $\mathcal{I}^{\alpha}$ is not a unique solution of (4.15) and there may be more than one set of allocation matrices of $\mathcal{I}^{\alpha}$ whose elements maximize (4.15). Let $\mathbb{I}^{\alpha}=\left\{\mathcal{I}_{i}^{\alpha}, 1 \leq i \leq\left|\mathbb{I}^{\alpha}\right|\right\}$ denote the set of all distinguished solutions of (4.15). Obviously $\left|\mathbb{I}^{\alpha}\right|<\infty$, since set $\mathcal{I}$ is finite. Note that each solution $\mathcal{I}_{i}^{\alpha}=\left\{I_{s, i}^{\alpha}, s \in \mathcal{S}\right\}, 1 \leq i \leq\left|\mathbb{I}^{\alpha}\right|$ is corresponding to a deterministic policy with the average transmission rate vector

$$
R_{i}^{\alpha}=\left(\sum_{s \in \mathcal{S}} \pi_{s}\left(C_{s} \circledast I_{s, i}^{\alpha}\right) \underline{1}_{K}^{\mathrm{T}}\right)^{\mathrm{T}}
$$

and therefore, according to (4.4) each solution of (4.15) is associated with a vertex of polytope $\mathcal{P}$. Since there are $\left|\mathbb{I}^{\alpha}\right|$ distinguished solution of (4.15), $\left|\mathbb{I}^{\alpha}\right|$ vertices of polytope $\mathcal{P}$ are on the hyperplane associated to half space (4.10), i.e.,

$$
\alpha \overline{E[A(t)]^{\mathrm{T}}}=\sum_{s \in \mathcal{S}} \pi_{s} \max _{I \in \mathcal{I}}\left(\alpha\left(C_{s} \circledast I\right) \underline{1}_{K}^{\mathrm{T}}\right)
$$

Therefore, each face of $\mathcal{P}$ defined by hyperplane (C.2) is represented by $F_{\alpha}=$ conv.hull ${ }_{1 \leq i \leq|\mathbb{I}| \alpha} R_{i}^{\alpha}$. Since polytope $\mathcal{P}$ is full dimensional, according to Definition A.5, each facet of $\mathcal{P}$ must be of dimension $N-1$. In the following, we will show that the dimension of face $F_{\alpha}$ is less than $N-1$ if $\alpha \in \mathbb{R}_{+}^{N}-V$ and therefore the hyperplane (C.2) associated to such $\alpha$ is not a facet defining hyperplane. 
Consider a vector $\alpha \in \mathbb{R}_{+}^{N}-V$. For $\alpha$ we have the following property:

$$
\begin{aligned}
& \exists\left(\mathcal{U} \subset \mathcal{N}, \mathcal{U} \neq \varnothing, \alpha_{\mathcal{U}} \neq \underline{0}_{|\mathcal{U}|}, \alpha_{\mathcal{U}^{c}} \neq \underline{0}_{|\mathcal{U}|}\right): \\
& \quad \forall\left(i \in \mathcal{U}, j \in \mathcal{U}^{c}, m, n \in \mathcal{M}, \alpha_{i}, \alpha_{j}, m, n \neq 0\right) \alpha_{i} m \neq \alpha_{j} n
\end{aligned}
$$

In other words, there exists a partitioning of vector $\alpha$ into two (sub)vectors $\alpha_{\mathcal{U}}$ and $\alpha_{\mathcal{U}}$ such that no non-zero element of $\alpha_{\mathcal{U}}$ is proportional to no non-zero element of $\alpha_{\mathcal{U}}$ by the ratio of two non-zero elements in $\mathcal{M}$. Assume that $\mathcal{U}_{0}$ denotes the subset $\mathcal{U}$ satisfying (C.3). Now, consider inequality (4.10). The right hand side of (4.10) can also be expressed as

$$
\sum_{s \in \mathcal{S}} \pi_{s} \max _{I \in \mathcal{I}}\left(\alpha\left(C_{s} \circledast I\right) \underline{1}_{K}^{\mathrm{T}}\right)=\sum_{s \in \mathcal{S}} \pi_{s} \sum_{k=1}^{K} \max _{J \in \mathcal{J}}\left\langle\alpha,\left(\left(C_{s}^{\downarrow k}\right)^{\mathrm{T}} \circledast J\right)\right\rangle,
$$

where $C_{s}^{\downarrow k}$ is the $k^{\prime}$ th column of matrix $C_{s}$ and $\mathcal{J}$ is the set of all binary vectors of size $N$ with $\left\langle J, \underline{1}_{N}\right\rangle=1$, i.e.,

$$
\mathcal{J}:=\left\{J=\left(J_{1}, J_{2}, \ldots, J_{N}\right) \mid J_{n} \in\{0,1\} \forall n \in \mathcal{N},\left\langle J, \underline{1}_{N}\right\rangle=1\right\}
$$

Each $J_{s}^{k}(\alpha)=\arg \max _{J \in \mathcal{J}}\left\langle\alpha,\left(\left(C_{s}^{\downarrow k}\right)^{\mathbf{T}} \circledast J\right)\right\rangle$ is corresponding to the $k^{\prime}$ th column of an $I_{s}^{\alpha}$ in some $\mathcal{I}^{\alpha} \in \mathbb{I}^{\alpha}$. Note that $\max _{J \in \mathcal{J}}\left\langle\alpha,\left(\left(C_{s}^{\downarrow k}\right)^{\mathrm{T}} \circledast J\right)\right\rangle \geq 0$. There are $K|\mathcal{S}|$ of such maximization terms in the right hand side of (C.4). Each of the $K|\mathcal{S}|$ maximization terms may have multiple solutions (non-unique solutions). Suppose that $\psi_{s}^{k}(\alpha)$ denotes the set of all distinct solutions to $J_{s}^{k}(\alpha)=\arg \max _{J \in \mathcal{J}}\left\langle\alpha,\left(\left(C_{s}^{\downarrow k}\right)^{\mathbf{T}} \circledast J\right)\right\rangle$, i.e.,

$$
\psi_{s}^{k}(\alpha)=\left\{J_{s}^{k}(\alpha)=\arg \max _{J \in J}\left\langle\alpha,\left(\left(C_{s}^{\downarrow k}\right)^{\mathrm{T}} \circledast J\right)\right\rangle \forall s \in \mathcal{S}, k \in \mathcal{K}\right\}
$$

For a given $s \in \mathcal{S}, k \in \mathcal{K}$ with $\left\langle\alpha,\left(\left(C_{s}^{\downarrow k}\right)^{\mathrm{T}} \circledast J_{s}^{k}(\alpha)\right)\right\rangle>0$, we can easily observe that for all the elements of $\psi_{s}^{k}(\alpha)$ either we have $\sum_{u \in \mathcal{U}_{0}} J_{s, u}^{k}(\alpha)=1$ or $\sum_{u \in \mathcal{U}_{0}^{c}} J_{s, u}^{k}(\alpha)=1$. This result comes directly from (C.3) and states that for a given $s \in \mathcal{S}, k \in \mathcal{K}$, for all the solutions of $J_{s}^{k}(\alpha)=\arg \max _{J \in \mathcal{J}}\left\langle\alpha,\left(\left(C_{s}^{\downarrow k}\right)^{\mathrm{T}} \circledast J\right)\right\rangle$ with $\max _{J \in \mathcal{J}}\left\langle\alpha,\left(\left(C_{s}^{\downarrow k}\right)^{\mathbf{T}} \circledast J\right)\right\rangle>0$, the index associated to element " 1 " of $J_{s}^{k}(\alpha)$ is either in $\mathcal{U}_{0}$ or $\mathcal{U}_{0}^{c}$. In other words, it is not possible to have the index of element " 1 " in $\mathcal{U}_{0}$ for some solutions and in $\mathcal{U}_{0}^{c}$ for other solutions of $J_{s}^{k}(\alpha)=\arg \max _{J \in \mathcal{J}}\left\langle\alpha,\left(\left(C_{s}^{\downarrow k}\right)^{\mathrm{T}} \circledast J\right)\right\rangle$. Using this property, we can partition set of all $\psi_{s}^{k}(\alpha)$ with $\left\langle\alpha,\left(\left(C_{s}^{\downarrow k}\right)^{\mathrm{T}} \circledast J_{s}^{k}(\alpha)\right)\right\rangle>0$ into two disjoint subsets. We denote the set of all solution sets $\psi_{s}^{k}(\alpha)$ with 
$\left\langle\alpha,\left(\left(C_{s}^{\downarrow k}\right)^{\mathrm{T}} \circledast J_{s}^{k}(\alpha)\right)\right\rangle>0$ and $\sum_{u \in \mathcal{U}_{0}} J_{s, u}^{k}(\alpha)=1$ as $\mathcal{A}_{\alpha}$ and set of all solution sets $\psi_{s}^{k}(\alpha)$ with $\left\langle\alpha,\left(\left(C_{s}^{\downarrow k}\right)^{\mathrm{T}} \circledast J_{s}^{k}(\alpha)\right)\right\rangle>0$ and $\sum_{u \in \mathcal{U}_{0}^{c}} J_{s, u}^{k}(\alpha)=1$ as $\mathcal{B}_{\alpha}$. We introduce $N$ dimensional vectors $\alpha_{\mathcal{U}_{0}}^{\prime}$ and $\alpha_{\mathcal{U}_{0}^{c}}^{\prime}$ as follows.

$$
\begin{gathered}
\alpha_{\mathcal{U}_{0}}^{\prime}=\left(\alpha_{1}^{\prime}, \alpha_{2}^{\prime}, \ldots, \alpha_{N}^{\prime}\right): \alpha_{n}^{\prime}= \begin{cases}\alpha_{n} & \text { if } n \in \mathcal{U} \\
0 & \text { if } n \notin \mathcal{U}\end{cases} \\
\alpha_{\mathcal{U}_{0}^{c}}^{\prime}=\left(\alpha_{1}^{\prime}, \alpha_{2}^{\prime}, \ldots, \alpha_{N}^{\prime}\right): \alpha_{n}^{\prime}= \begin{cases}\alpha_{n} & \text { if } n \in \mathcal{U}^{c} \\
0 & \text { if } n \notin \mathcal{U}^{c}\end{cases}
\end{gathered}
$$

Now, let us introduce the following two hyperplanes.

$$
\begin{aligned}
& \alpha_{\mathcal{U}_{0}}^{\prime} \overline{E\left[A_{\mathcal{U}}(t)\right]^{\mathrm{T}}}=\sum_{k, s: \psi_{s}^{k}(\alpha) \in \mathcal{A}_{\alpha}} \pi_{s} \max _{J \in \mathcal{J}}\left\langle\alpha,\left(\left(C_{s}^{\downarrow k}\right)^{\mathbf{T}} \circledast J\right)\right\rangle \\
& \alpha_{\mathcal{U}_{0}^{c}}^{\prime} \overline{E\left[A_{\mathcal{U}^{c}}(t)\right]^{\mathrm{T}}}=\sum_{k, s: \psi_{s}^{k}(\alpha) \in \mathcal{B}_{\alpha}} \pi_{s} \max _{J \in \mathcal{J}}\left\langle\alpha,\left(\left(C_{s}^{\downarrow k}\right)^{\mathrm{T}} \circledast J\right)\right\rangle
\end{aligned}
$$

In the following, we will prove that all the vertices $R_{i}^{\alpha}, 1 \leq i \leq\left|\mathbb{I}^{\alpha}\right|$ in (C.1) satisfy both (C.7) and (C.8). In other words, all the vertices $R_{i}^{\alpha}$ are located on both hyperplanes (C.7) and (C.8) and therefore on the intersection of both. The hyperplanes of (C.7) and (C.8) are in an $N$ dimensional space whose dimensions are at most $N-1$. Thus, dimension of their intersection is at most $N-2$. In other words, $\operatorname{dim} F_{\alpha}=\operatorname{dim}\left(\right.$ conv.hull $\left.l_{1 \leq i \leq|\mathbb{1} \alpha|} R_{i}^{\alpha}\right)<N-1$. Therefore $F_{\alpha}$ is not a facet for $\mathcal{P}$.

Note that columns of any allocation matrix $I_{s}^{\alpha}$ are vectors $J_{s}^{k}(\alpha) \in \psi_{s}^{k}(\alpha)$ for all $k \in \mathcal{K}$. Consider a particular transmission rate vector $R_{i}^{\alpha}$ (equation (C.1)) located on hyperplane (C.2). As we discussed before, each allocation matrix $I_{s, i}^{\alpha}$ in (C.1) is created by concatenation of a set of vectors $J_{s, i}^{k}(\alpha) \in \psi_{s}^{k}(\alpha)$ as the columns of $I_{s, i}^{\alpha}$. Now, we can check that all the vertices $R_{i}^{\alpha}, 1 \leq i \leq\left|\mathbb{I}^{\alpha}\right|$ in (C.1) satisfy both (C.7) 
and (C.8) as follows.

$$
\begin{aligned}
& \alpha_{\mathcal{U}_{0}}^{\prime}\left(\sum_{s \in \mathcal{S}} \pi_{s}\left(C_{s} \circledast I_{s, i}^{\alpha}\right) \underline{1}_{K}^{\mathrm{T}}\right) \\
& \quad=\sum_{s \in \mathcal{S}} \pi_{s} \sum_{k=1}^{K}\left\langle\alpha_{\mathcal{U}_{0}}^{\prime},\left(\left(C_{s}^{\downarrow k}\right)^{\mathrm{T}} \circledast J_{s, i}^{k}(\alpha)\right)\right\rangle=\sum_{k, s: \psi_{s}^{k}(\alpha) \in \mathcal{A}_{\alpha}} \pi_{s} \max _{J \in \mathcal{J}}\left\langle\alpha,\left(\left(C_{s}^{\downarrow k}\right)^{\mathrm{T}} \circledast J\right)\right\rangle
\end{aligned}
$$

and

$$
\begin{aligned}
& \alpha_{\mathcal{U}_{0}}^{\prime}\left(\sum_{s \in \mathcal{S}} \pi_{s}\left(C_{s} \circledast I_{s, i}^{\alpha}\right) \underline{1}_{K}^{\mathrm{T}}\right) \\
& \quad=\sum_{s \in \mathcal{S}} \pi_{s} \sum_{k=1}^{K}\left\langle\alpha_{\mathcal{U}_{0}^{c}}^{\prime},\left(\left(C_{s}^{\downarrow k}\right)^{\mathrm{T}} \circledast J_{s, i}^{k}(\alpha)\right)\right\rangle=\sum_{k, s: \psi_{s}^{k}(\alpha) \in \mathcal{B}_{\alpha}} \pi_{s} \max _{J \in \mathcal{J}}\left\langle\alpha,\left(\left(C_{s}^{\downarrow k}\right)^{\mathrm{T}} \circledast J\right)\right\rangle
\end{aligned}
$$

Thus, all the vertex (C.1) satisfy both (C.7) and (C.8) and therefore their intersection. This shows that $F_{\alpha}$ has dimension less than $N-1$ and therefore is not a facet of $\mathcal{P}$.

\section{C.2 Proof of Lemma 4.6}

Proof: Consider vector $\alpha=\left(\alpha_{1}, \alpha_{2}, \ldots, \alpha_{N}\right) \in V$. From (4.16), it is obvious that any vector derived by the multiplication of a positive scalar to $\alpha$ also belongs to $V$, i.e.,

$$
\alpha \in V \Longrightarrow q \alpha \in V \quad \forall q \in \mathbb{R}_{+}
$$

Also note that vectors $\alpha$ and $q \alpha$ will result in the same inequalities in (4.10) and therefore the same face defining hyperplanes of polytope $\mathcal{P}$. Thus, we say that $\alpha$ and $q \alpha$ are equivalent and we write $\alpha \equiv q \alpha$.

Consider a vector $\alpha \in V$. Vector $\alpha$ may be a zero vector in which case it does not contributes to a face of $\mathcal{P}$ as the inequality (4.10) will result in an obvious equality $0=0$. Hence, we assume that $\alpha$ is not a zero vector.

Now, we perform the following process on vector $\alpha$. Pick a non-zero elements 
of $\alpha$, let say $\alpha_{e_{1}}$ and form $\alpha_{\left\{e_{1}\right\}}$ and $\alpha_{\mathcal{N}-\left\{e_{1}\right\}}$. The size of these vectors are 1 and $N-1$, respectively. According to (4.16), the vector $\alpha_{\mathcal{N}-\left\{e_{1}\right\}}$ should be either a zero vector in which case we stop or there should be a non-zero element $\alpha_{e_{2}}$ in $\alpha_{\mathcal{N}-\left\{e_{1}\right\}}$ and non-zero $m_{1}, n_{1} \in \mathcal{M}$ such that $\alpha_{e_{2}}=\alpha_{e_{1}} \frac{m_{1}}{n_{1}}$. If so, we will continue and form $\alpha_{\left\{e_{1}, e_{2}\right\}}$ and $\alpha_{\mathcal{N}-\left\{e_{1}, e_{2}\right\}}$. According to (4.16), the vector $\alpha_{\mathcal{N}-\left\{e_{1}, e_{2}\right\}}$ is either a zero vector in which case we will stop or there should exist a non-zero $\alpha_{e_{3}} \in \alpha_{\mathcal{N}-\left\{e_{1}, e_{2}\right\}}$ and non-zero $m_{2}, n_{2}$ such that either $\alpha_{e_{3}}=\alpha_{e_{1}} \frac{m_{2}}{n_{2}}$ or $\alpha_{e_{3}}=\alpha_{e_{2}} \frac{m_{2}}{n_{2}}=\alpha_{e_{1}} \frac{m_{1}}{n_{1}} \frac{m_{2}}{n_{2}}$. We repeat this procedure until either for some $\nu<N$ the vector $\alpha_{\mathcal{N}-\left\{e_{1}, e_{2}, \ldots, e_{\nu}\right\}}$ is a zero vector or we form vector $\alpha_{\left\{e_{1}, e_{2}, \ldots, e_{N}\right\}}$ in which case $\nu=N$. In both cases, we can see that all the non-zero elements of $\alpha$ may be expressed by the multiplication of $\alpha_{1}$ and the rational numbers derived by non-zero elements of set $\mathcal{M}$. By dividing the vector $\alpha$ by scalar $\alpha_{e_{1}}$ and multiplying it by $\prod_{j=1}^{\nu-1} n_{j}$, we will obtain an equivalent vector for $\alpha$ named $\beta$ whose elements belong to the set of all integers which were derived by multiplication of $N-1$ elements of set $\mathcal{M}$, i.e.,

$$
\alpha \equiv \beta, \beta_{n} \in \mathcal{W}=\left\{z \in \mathbb{Z}_{+} \mid z=\prod_{j=1}^{N-1} m_{j}, m_{j} \in \mathcal{M}\right\} \quad \forall n \in\{1,2, \ldots, N\}
$$

Therefore, each vector $\alpha \in V$ is equivalent to a vector $\beta$ whose elements come from set $\mathcal{W}$. We define the set of all $\beta$ vectors with the above property as $\widehat{V}$. Note that $\widehat{V} \subseteq \mathcal{W}^{N}$ and is a finite set since $\mathcal{W}$ is finite. Thus, for each vector $\alpha \in V$ there exists an equivalent vector $\beta \in \widehat{V}$ and the result follows.

\section{C.3 Proof of Theorem 4.2}

Proof: We will start with the Lyapunov function evaluation. we will use the quadratic function (A.1) as our Lyapunov function. The Lyapunov drift for two successive time slots has the following form.

$$
\begin{aligned}
& D(t+1)=E[L(X(t+1))-L(X(t)) \mid X(t)] \\
& =E\left[\sum_{n=1}^{N} X_{n}^{2}(t+1)-X_{n}^{2}(t) \mid X(t)\right]=E\left[\sum_{n=1}^{N}\left(X_{n}(t+1)-X_{n}(t)\right)^{2} \mid X(t)\right] \\
& +2 E\left[\sum_{n=1}^{N} X_{n}(t)\left(X_{n}(t+1)-X_{n}(t)\right) \mid X(t)\right]
\end{aligned}
$$


For the the first term we have

$$
\begin{aligned}
E & {\left[\sum_{n=1}^{N}\left(X_{n}(t+1)-X_{n}(t)\right)^{2} \mid X(t)\right] } \\
& =E\left[\sum_{n=1}^{N}\left(A_{n}(t+1)-\sum_{k=1}^{K} H_{n, k}(t+1)\right)^{2} \mid X(t)\right]=E\left[\sum_{n=1}^{N} A_{n}^{2}(t+1) \mid X(t)\right] \\
& -2 E\left[\sum_{n=1}^{N} \sum_{k=1}^{K} A_{n}(t+1) H_{n, k}(t+1) \mid X(t)\right]+E\left[\sum_{n=1}^{N}\left(\sum_{k=1}^{K} H_{n, k}(t+1)\right)^{2} \mid X(t)\right] \\
& \leq N A_{\max }^{2}+\sum_{n=1}^{N} E\left[\left(\sum_{k=1}^{K} H_{n, k}(t+1)\right)^{2} \mid X(t)\right]
\end{aligned}
$$

For the second term in (C.13) we have

$$
\begin{aligned}
E\left[\sum_{n=1}^{N} X_{n}(t)\left(X_{n}(t+1)-X_{n}(t)\right) \mid X(t)\right] \\
=E\left[\sum_{n=1}^{N} X_{n}(t)\left(A_{n}(t+1)-\sum_{k=1}^{K} H_{n, k}(t+1)\right) \mid X(t)\right] \\
=\sum_{n=1}^{N} X_{n}(t) E\left[A_{n}(t+1)\right]-\sum_{n=1}^{N} E\left[X_{n}(t) \sum_{k=1}^{K} H_{n, k}(t+1) \mid X(t)\right]
\end{aligned}
$$

Therefore, the Lyapunov drift $D(t+1)$ can be bounded by

$$
\begin{aligned}
D(t+1) \leq & N A_{\max }^{2}+\sum_{n=1}^{N} E\left[\left(\sum_{k=1}^{K} H_{n, k}(t+1)\right)^{2} \mid X(t)\right] \\
& +2 \sum_{n=1}^{N} X_{n}(t) E\left[A_{n}(t+1)\right]-2 \sum_{n=1}^{N} E\left[X_{n}(t) \sum_{k=1}^{K} H_{n, k}(t+1) \mid X(t)\right]
\end{aligned}
$$


In the following, we show that the Lyapunov drift in (C.16) is bounded as follows.

$$
\begin{aligned}
D(t+1) & \leq N A_{\text {max }}^{2}+\sum_{n=1}^{N} E\left[\left(\sum_{k=1}^{K} C_{n, k}(t+1) I_{n, k}(t+1)\right)^{2} \mid X(t)\right] \\
& +2 \sum_{n=1}^{N} X_{n}(t) E\left[A_{n}(t+1)\right]-2 \sum_{n=1}^{N} E\left[X_{n}(t) \sum_{k=1}^{K} C_{n, k}(t+1) I_{n, k}(t+1) \mid X(t)\right]
\end{aligned}
$$

To prove (C.17), note that for each queue $n \in \mathcal{N}$ one of the following conditions is satisfied.

- $\sum_{k=1}^{K} H_{n, k}(t+1)=\sum_{k=1}^{K} C_{n, k}(t+1) I_{n, k}(t+1)$ : In this case, we can easily check that for all $n \in \mathcal{N}$

$$
\begin{aligned}
& E\left[X_{n}^{2}(t+1)-X_{n}^{2}(t) \mid X(t)\right] \leq N A_{\max }^{2} \\
& \quad+E\left[\left(\sum_{k=1}^{K} C_{n, k}(t+1) I_{n, k}(t+1)\right)^{2} \mid X(t)\right] \\
& \quad+2 X_{n}(t) E\left[A_{n}(t+1)\right]-2 E\left[X_{n}(t) \sum_{k=1}^{K} C_{n, k}(t+1) I_{n, k}(t+1) \mid X(t)\right]
\end{aligned}
$$

- $\sum_{k=1}^{K} H_{n, k}(t+1)<\sum_{k=1}^{K} C_{n, k}(t+1) I_{n, k}(t+1)$ : In this case, $\sum_{k=1}^{K} H_{n, k}(t+1)=$ $X_{n}(t)$ and $X_{n}(t+1)=A_{n}(t+1)$ as there are not enough packets in queue $n$ to be server at time slot $t+1$. It is not hard to check that inequality (C.18) is also satisfied in this case.

According to the above discussion, we can observe that inequality (C.18) is satisfied for all the queues and therefore (C.17) follows.

$$
\begin{aligned}
& \text { Using the the fact that } \sum_{k=1}^{K} C_{n, k}(t+1) I_{n, k}(t+1) \geq 0 \text {, we get the following inequality. } \\
& \sum_{n=1}^{N}\left(\sum_{k=1}^{K} C_{n, k}(t+1) I_{n, k}(t+1)\right)^{2} \leq\left(\sum_{n=1}^{N} \sum_{k=1}^{K} C_{n, k}(t+1) I_{n, k}(t+1)\right)^{2} \leq(M K)^{2}
\end{aligned}
$$


Hence, the Lyapunov drift (C.17) is bounded by

$$
\begin{aligned}
D(t+1) \leq N A_{\max }^{2}+(M K)^{2}+2 \sum_{n=1}^{N} X_{n}(t) E\left[A_{n}(t+1)\right] \\
-2 \sum_{n=1}^{N} E\left[X_{n}(t) \sum_{k=1}^{K} C_{n, k}(t+1) I_{n, k}(t+1) \mid X(t)\right]
\end{aligned}
$$

By conditioning the last term of (C.20) on the channel state at time slot $t+1$, we will have

$$
\begin{aligned}
& D(t+1) \leq N A_{\max }^{2}+(M K)^{2} \\
&+2 E\left[X(t)\left(A^{\mathrm{T}}(t+1)-\sum_{s \in \mathcal{S}} \pi_{s}\left(C_{s} \circledast I(t+1)\right) \underline{1}_{K}^{\mathrm{T}}\right) \mid X(t)\right]
\end{aligned}
$$

Note that the allocation matrix $I$ in (C.21) depends on the selected policy. According to (4.20), we can see that by selecting MW policy, the second term of (C.21) will be minimized and therefore the right hand side term in (C.21) will be minimized over all the existing server allocation policies. In other words, for MW policy and any arbitrary server allocation policy $\Delta$ we have

$$
\begin{aligned}
& D^{M W}(t+1) \leq N A_{\max }^{2}+(M K)^{2} \\
& \quad+2 E\left[X(t)\left(A^{\mathrm{T}}(t+1)-\sum_{s \in \mathcal{S}} \pi_{s}\left(C_{s} \circledast I^{M W}(t+1)\right) \underline{1}_{K}^{\mathrm{T}}\right) \mid X(t)\right] \\
& \leq N A_{\max }^{2}+(M K)^{2} \\
& \quad+2 E\left[X(t)\left(A^{\mathrm{T}}(t+1)-\sum_{s \in \mathcal{S}} \pi_{s}\left(C_{s} \circledast I^{\Delta}(t+1)\right) \underline{1}_{K}^{\mathrm{T}}\right) \mid X(t)\right]
\end{aligned}
$$

where $I^{M W}(t+1)$ and $I^{\Delta}(t+1)$ are the allocation matrices of policies MW and $\Delta$, respectively and $D^{M W}(t+1)$ is the Lyapunov drift of policy $\mathrm{MW}$ at time slot $t+1$.

It is important to note that the set of inequalities in (4.21) determines an open polytope $\mathcal{P}^{\prime}$ for which we have $\mathcal{P}^{\prime}=\mathcal{P}$ - bound $(\mathcal{P})$. In fact, Theorem 4.2 states that if the vector $E[A(t)]$ at each time slot $t$ is strictly inside polytope $\mathcal{P}$ then the system is stable. Now, consider an MQMS system with arrival processes for which we have $E[A(t)] \in \mathcal{P}^{\prime}$ at each time slot $t$. Suppose that $\delta>0$ is a positive real number such that $E[A(t)]+\delta \underline{1}_{N} \in \operatorname{bound}(\mathcal{P})$. Therefore, $E[A(t)]+\delta \in \mathcal{P} \forall t$. According to 
our discussion about randomized policies, there exists a randomized policy $I^{\text {rnd }}=$ $\left\{I^{r n d}(t)\right\}_{t=1}^{\infty}$ for which we will have

$$
E\left[\left(\sum_{s \in \mathcal{S}} \pi_{s}\left(C_{s} \circledast I^{r n d}(t+1)\right) \underline{1}_{K}^{\mathrm{T}}\right)^{\mathrm{T}}\right]=E[A(t+1)]+\delta \underline{1}_{N}
$$

Therefore,

$$
E\left[X(t)\left(A^{\mathrm{T}}(t+1)-\sum_{s \in \mathcal{S}} \pi_{s}\left(C_{s} \circledast I^{\Delta}(t+1)\right) \underline{1}_{K}^{\mathrm{T}}\right) \mid X(t)\right]=-\delta \sum_{n=1}^{N} X_{n}(t)
$$

Putting together from (C.21) to (C.24), we conclude that

$$
D^{M W}(t+1) \leq N A_{\max }^{2}+(M K)^{2}-2 \delta \sum_{n=1}^{N} X_{n}(t)
$$

and according to (A.2) the stability of MQMS system is demonstrated. It can be also inferred that $\delta=\min _{t, \alpha \in \hat{V}}\left\{\frac{1}{\sum_{i=1}^{N} \alpha_{i}}\left(\sum_{s \in \mathcal{S}} \pi_{s} \max _{I \in \mathcal{I}}\left(\alpha\left(C_{s} \circledast I\right) \underline{1}_{K}^{\mathrm{T}}\right)-\alpha E[A(t)]^{\mathrm{T}}\right)\right\}$. 


\section{Appendix D}

\section{Proofs of the Results of Chapter 5}

\section{D.1 Proof of Lemma 5.1}

Proof: Let $I^{(\tilde{\pi})}(t)$ denote the employed matching after applying the balancing reallocation. According to the definition of balancing server reallocation, a server reallocation at time slot $t$ results in a queue length vector $\tilde{x}^{\prime}(t)$ that satisfies either condition $\mathbf{C 1}$ or $\mathbf{C 2}$. Therefore, we consider the following two cases:

Case 1: Condition $\mathbf{C} 1$ is satisfied at time slot $t$. Thus, $\tilde{x}_{n}^{\prime}(t) \leq x_{n}^{\prime}(t)$ for all $n=1,2, \ldots, N$ and there exists $m \in\{1,2, \ldots, N\}$ such that $0 \leq \tilde{x}_{m}^{\prime}(t)<x_{m}^{\prime}(t)$. Therefore, there exists no queue that was served by $\pi$ but not by $\tilde{\pi}$. Also queue $m$ which was not receiving service by $\pi$ at time slot $t$, is now receiving service after applying the balancing server reallocation. Therefore, $x_{m}(t-1) \sum_{k=1}^{K} c_{m, k}(t) I_{m, k}^{(\pi)}(t)=$ $0, x_{m}(t-1) \sum_{k=1}^{K} c_{m, k}(t) I_{m, k}^{(\bar{\pi})}(t)=x_{m}(t-1)>0$ and

$$
\sum_{n=1, n \neq m}^{N} x_{n}(t-1) \sum_{k=1}^{K} c_{n, k}(t) I_{n, k}^{(\pi)}(t) \leq \sum_{n=1, n \neq m}^{N} x_{n}(t-1) \sum_{k=1}^{K} c_{n, k}(t) I_{n, k}^{(\tilde{\pi})}(t) .
$$

Therefore, $\mathrm{MW}_{\pi}(t)<\mathrm{MW}_{\tilde{\pi}}(t)$.

Case 2: Condition C2 is satisfied at time $t$. In this case, by using policy $\pi$ at time slot $t$ queue $n$ is receiving service but queue $m$ is not. In contrast, by using policy $\tilde{\pi}$, at time slot $t$ queue $m$ is receiving service but queue $n$ is not. Therefore, we have

$$
\mathrm{MW}_{\tilde{\pi}}(t)-\mathrm{MW}_{\pi}(t)=x_{m}(t-1)-x_{n}(t-1)>0
$$

Therefore, $\mathrm{MW}_{\pi}(t)<\mathrm{MW}_{\tilde{\pi}}(t)$. By considering cases 1 and 2 the lemma follows. 


\section{D.2 Proof of Lemma 5.2}

Proof: Without loss of generality, we may convert the bipartite graph $G_{t}$ to a complete weighted bipartite graph $G_{t}^{\prime}$. This is done by adding some vertices and edges of zero weight as necessary. In particular, if $N>K$, we will add $N-K$ servers on the right hand side with edges of weight " 0 " to each queue (each vertex on the left hand side). It is like the case that we add some servers with service rate 0 to the system. If $N<K$, we will add $K-N$ queues on the left hand side with edges of weight " 0 " to each server (each vertex on the right hand side). It is like the case that we add some queues which are disconnected from all the servers. Thus, we deal with a complete bipartite graph with $\max \{N, K\}$ vertices on each side. We denote the sets of vertices on each part of $G_{t}^{\prime}$ by $\mathcal{N}^{\prime}$ and $\mathcal{K}^{\prime}$, respectively and the set of edges by $\mathcal{E}^{\prime}$. Consequently, a policy $\pi$ is defined as $\pi=\left\{I^{(\pi)}(t)\right\}_{t=1}^{\infty}$ where $I^{(\pi)}(t)$ is a perfect matching in the complete bipartite graph $G_{t}^{\prime}$. Note that a maximum weight perfect matching $I^{(\mathrm{MWM})}(t)$ in the complete bipartite graph $G_{t}^{\prime}$ is the same as the maximum weighted matching in graph $G_{t}$ if we remove the added edges of weight " 0 " from matching $I^{(\mathrm{MWM})}(t)$.

Consider now a policy $\pi$ which is employing perfect matching $I^{(\pi)}(t)$ at time slot $t$. Suppose that $I^{(\pi)}(t)$ is not a maximum weight perfect matching on graph $G_{t}^{\prime}$, i.e., $\mathrm{MW}_{\pi}(t)<\mathrm{MW}_{\mathrm{MWM}}(t)$. Also, consider a maximum weight perfect matching $I^{(\mathrm{MWM})}(t)$ at time slot $t$. Now, consider these two matchings on $G_{t}^{\prime}=\left(\mathcal{N}^{\prime}, \mathcal{K}^{\prime}, \mathcal{E}^{\prime}\right)$. Each of $I^{(\pi)}(t)$ and $I^{(\mathrm{MWM})}(t)$ corresponds to a distinct sub-graph of $G_{t}^{\prime}$ namely $G_{t}^{(\pi)}=\left(\mathcal{N}^{\prime}, \mathcal{K}^{\prime}, \mathcal{E}^{(\pi)}\right)$ and $G_{t}^{\prime(\mathrm{MWM})}=\left(\mathcal{N}^{\prime}, \mathcal{K}^{\prime}, \mathcal{E}^{(\mathrm{MWM})}\right)$, respectively. We now build two directed weighted sub-graphs $D_{t}^{(\pi)}$ and $D_{t}^{(\mathrm{MWM})}$ as follows: $D_{t}^{(\pi)}$ is the same as $G_{t}^{(\pi)}$ with all the edges directed from $\mathcal{N}^{\prime}$ to $\mathcal{K}^{\prime}$ with the same edge weights as $G_{t}^{\prime(\pi)} . D_{t}^{(\mathrm{MWM})}$ is the same as ${G^{\prime}}_{t}^{(\mathrm{MWM})}$ with all the edges directed from $\mathcal{K}^{\prime}$ to $\mathcal{N}^{\prime}$ with edge weights equal to the negative of edge weights of $G_{t}^{(\mathrm{MWM})}$. Now, consider graph $U=D_{t}^{(\pi)} \cup D_{t}^{(\mathrm{MWM})}$, i.e., the union of the sub-graphs $D_{t}^{(\pi)}$ and $D_{t}^{(\mathrm{MWM})}$. Graph $U$ is also the union of some even cycles denoted by $L$. This is directly concluded from the fact that $D_{t}^{(\pi)}$ and $D_{t}^{(\mathrm{MWM})}$ are each perfect matchings of $G_{t}^{\prime}$ and thus each vertex is incident to an incoming edge and an outgoing edge. Furthermore, for the weight of $U$ we have

$$
w(U)=\sum_{\ell \in L} w(\ell)=\mathrm{MW}_{\pi}(t)-\mathrm{MW}_{\mathrm{MWM}}(t)<0 .
$$

Therefore, there must exist a negative cycle in $U$ namely $\ell^{\star}$. Cycle $\ell^{\star}$ is an 


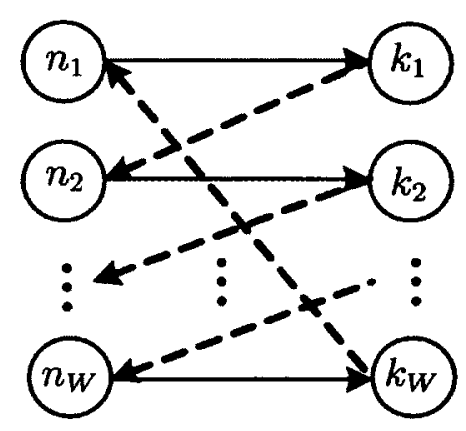

Figure D.1: The negative cycle $\ell^{\star}$

even cycle that contains an even number of nodes let say $2 W$ nodes ( $W$ nodes from $\mathcal{N}^{\prime}$ and $W$ nodes from $\mathcal{K}^{\prime}$ ) and also $2 W$ edges. Let us denote the nodes of set $\mathcal{N}^{\prime}$ and $\mathcal{K}^{\prime}$ that contribute in $\ell^{\star}$ by $n_{1}, n_{2}, \ldots, n_{W}$ and $k_{1}, k_{2}, \ldots, k_{W}$, respectively. Thus, cycle $\ell^{\star}$ can be represented by the sequence of its edges as $\ell^{\star}=$ $e_{n_{1}, k_{1}}, e_{k_{1}, n_{2}}, e_{n_{2}, k_{2}}, e_{k_{2}, n_{3}}, \ldots, e_{k_{W-1}, n_{W}}, e_{n_{W}, k_{W}}, e_{k_{W}, n_{1}}$ (see Figure D.1).

Note that edges $e_{k_{1}, n_{2}}, e_{k_{2}, n_{3}}, \ldots, e_{k_{W}, n_{1}}$ belong to $D_{t}^{(\mathrm{MWM})}$ and have negative weights while edges $e_{n_{1}, k_{1}}, e_{n_{2}, k_{2}}, \ldots, e_{n_{W}, k_{W}}$ belong to $D_{t}^{(\pi)}$ and have positive weights. We can also represent the edges of $\ell^{\star}$ by pairing the edges incident to each node $n_{1}, n_{2}, \ldots, n_{W}$ as follows; $\ell^{\star}=\left(e_{k_{W}, n_{1}}, e_{n_{1}, k_{1}}\right),\left(e_{k_{1}, n_{2}}, e_{n_{2}, k_{2}}\right), \ldots,\left(e_{k_{W-1}, n_{W}}, e_{n_{W}, k_{W}}\right)$. We label each pair of edges as follows: $r_{n_{1}}=\left(e_{k_{W}, n_{1}}, e_{n_{1}, k_{1}}\right), r_{n_{2}}=\left(e_{k_{1}, n_{2}}, e_{n_{2}, k_{2}}\right), \ldots, r_{n_{W}}=$ $\left(e_{k_{W-1}, n_{W}}, e_{n_{W}, k_{W}}\right)$. All $r_{n_{1}}, r_{n_{2}}, \ldots, r_{n_{W}}$ pairs in $\ell^{\star}$ have the following property: The weights associated to the edges of each pair $r_{n_{i}}, 1 \leq i \leq W$ is either $(0,0)$, $\left(0, x_{n_{i}}(t-1)\right),\left(-x_{n_{i}}(t-1), 0\right)$ or $\left(-x_{n_{i}}(t-1), x_{n_{i}}(t-1)\right)$. Accordingly, we will specify three types of edge pair as follows:

- Type 0 (T0): pairs with edge weights $(0,0)$ or $\left(-x_{n_{i}}(t-1), x_{n_{i}}(t-1)\right), 1 \leq$ $i \leq W$.

- Type 1 (T1): pairs with edge weights $\left(-x_{n_{i}}(t-1), 0\right), 1 \leq i \leq W$ and $x_{n_{i}}(t-1)>0$.

- Type 2 (T2): pairs with edge weights $\left(0, x_{n_{i}}(t-1)\right), 1 \leq i \leq W$ and $x_{n_{i}}(t-1)>$ 0 .

Since $\ell^{\star}$ is a negative cycle, there must exist a node $n_{j}$ with edge pairs $\left(e_{k_{j-1}, n_{j}}, e_{n_{j}, k_{j}}\right)=\left(-x_{n_{j}}(t-1), 0\right)$ (if $j=1,\left(e_{k_{W}, n_{1}}, e_{n_{1}, k_{1}}\right)=\left(-x_{n_{1}}(t-1), 0\right)$ ) and 


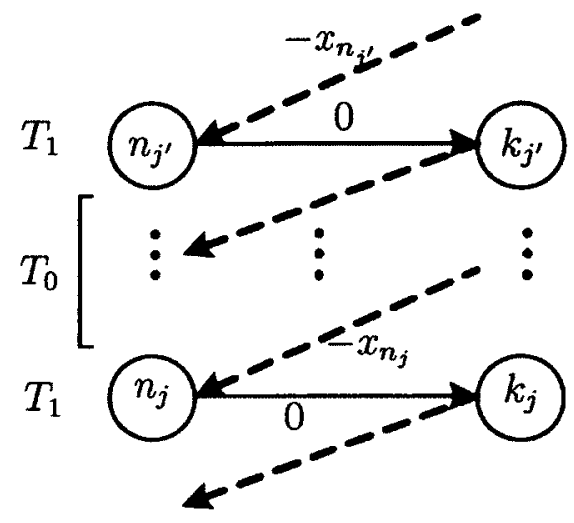

(a) Case 2

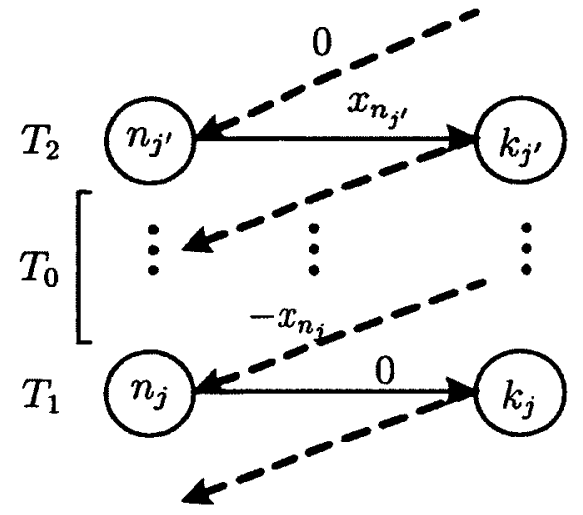

(b) Case 3

Figure D.2: Cases 2 and 3 in the proof of Lemma 5.2

$x_{n_{j}}(t-1)>0$. Obviously, the edge pair associated to node $n_{j}$ is of type $\mathbf{T} 1$. We now consider the following three cases:

Case 1: All the edge pairs other than $r_{n_{j}}$ are of type TO. In this case, by replacing the edges of $D_{t}^{(\pi)}$ by the edges of $D_{t}^{(\mathrm{MWM})}$ in the negative cycle $\ell^{\star}$ we obtain a server reallocation at time slot $t$. This server reallocation is balancing as in $\pi$ queue $n_{j}$ was not receiving service while after the server reallocation it is. Therefore, if $\tilde{x}^{\prime}(t)$ be the queue length vector after server reallocation we have $\tilde{x}^{\prime}(t) \leq x^{\prime}(t)$ (satisfying condition $\mathbf{C 1}$ ).

Case 2: If we go backward on the edge pairs of cycle $\ell^{\star}$ from $r_{n_{j}}$ and the first nonT0 pair is a $\mathbf{T 1}$ pair. Let $r_{n_{j^{\prime}}}$ be such a T1 edge pair. In other words, in cycle $\ell^{\star}$ the pairs $r_{n_{j^{\prime}}}$ and $r_{n_{j}}$ are of type $\mathbf{T 1}$ while other pairs between them are of type T0 (see Figure D.2(a)). In this case, by replacing the edges of $D_{t}^{(\pi)}$ by the edges of $D_{t}^{(\mathrm{MWM})}$ just for nodes $n_{j^{\prime}+1}$ to $n_{j}$ of cycle $\ell^{\star}$ and allocating $k_{j}$ to $n_{j^{\prime}}$ we obtain a server reallocation at time slot $t$. This server reallocation is balancing as in $\pi$ queue $n_{j}$ was not receiving service while after the server reallocation it is and also queue $n_{j^{\prime}}$ was not being serviced in $\pi$ but now may get service (depending on the weight of edge $\left.e_{k_{j}, n_{j^{\prime}}}\right)$ and the service of other queues is not disturbed. Therefore, if $\tilde{x}^{\prime}(t)$ be the queue length vector after server reallocation, we have $\tilde{x}^{\prime}(t) \leq x^{\prime}(t)$ and $\tilde{x}_{n_{j}}^{\prime}(t)<x_{n_{j}}^{\prime}(t)$ (satisfying condition $\mathbf{C 1}$ ).

Case 3: If we go backward on the edge pairs of cycle $\ell^{\star}$ from $r_{n_{j}}$ and the first nonT0 pair is a T2 pair. Let $r_{n_{j^{\prime}}}$ be such a T2 edge pair. In other words, in cycle $\ell^{\star}$, 
pair $r_{n_{j^{\prime}}}$ is of type T2 and $r_{n_{j}}$ is of type $\mathbf{T} 1$ while other pairs between them are of type To (see Figure D.2(b)). First of all, we claim that $x_{n_{j^{\prime}}}(t-1) \leq x_{n_{j}}(t-1)$. If $x_{n_{j^{\prime}}}(t-1)>x_{n_{j}}(t-1)$, by replacing the edges of $D_{t}^{(\mathrm{MWM})}$ by the edges of $D_{t}^{(\pi)}$ just for nodes $n_{j^{\prime}}$ to $n_{j-1}$ of the cycle $\ell^{\star}$ and not serving queue $n_{j}$, without disturbing the service of other queues we obtain a server reallocation at time slot $t$ with larger $\mathrm{MW}$ index than $\mathrm{MW}_{\mathrm{MWM}}(t)$ and this contradicts with the fact that MWM has the maximum $M W$ index at time slot $t$. Accordingly, we consider the following two sub-cases:

Sub-case 3.1: $x_{n_{j}}(t-1)>x_{n_{j^{\prime}}}(t-1)$. In this case, we replace the edges of $D_{t}^{(\pi)}$ by the edges of $D_{t}^{(\mathrm{MWM})}$ just for queues $n_{j^{\prime}+1}$ to $n_{j}$ of the cycle $\ell^{\star}$ and allocate server $k_{j}$ to queue $n_{j^{\prime}}$. Note that server $k_{j}$ may or may not serve queue $n_{j^{\prime}}$. We consider the worst case where it does not serve this queue $\left(w_{n_{j^{\prime}}, k_{j}}(t)=0\right)$. Thus, without disturbing the service of other queues we obtain a server reallocation with the following property: all the queues other than $n_{j}$ and $n_{j^{\prime}}$ have the same service as before; queue $n_{j}$ which was receiving zero service in $\pi$, now receives one packet service and queue $n_{j}$, which was receiving one packet service, now in the worst case looses its service. Therefore, this server reallocation is balancing as the queue length after server reallocation (denoted by $\tilde{x}^{\prime}(t)$ ) and the queue length after applying policy $\pi$ (denoted by $x^{\prime}(t)$ ) satisfy condition C2. So, $\tilde{x}^{\prime}(t) \preceq x^{\prime}(t)$ and therefore the applied server reallocation is a balancing one.

Sub-case 3.2: $x_{n_{j}}(t-1)=x_{n_{j^{\prime}}}(t-1)$. In this case, the sequence of edge pairs $r_{n_{j^{\prime}}}, \ldots, r_{n_{j}}$ contribute " 0 " in the calculation of $w(U)$. Therefore, we may treat them as a sequence of edge pairs of type T0. By doing so, we have a new negative cycle $\ell^{\prime \star}$ which is the same as $\ell^{\star}$ but the sequence of edge pairs $r_{n_{j^{\prime}}}, \ldots, r_{n_{j}}$ are replaces by the same number of edge pairs of type To. Note that cycle $\ell^{\prime \star}$ is a negative cycle with the same weight as $\ell^{\star}$. Therefore, there must exist an edge pair of type $\mathbf{T} 1$ in $\ell^{\prime \star}$ (In this case, it is not possible for $\ell^{\star \star}$ to have just edge pairs of type T0 as it results in $w\left(\ell^{\prime \star}\right)=0$ ) and all the cases of 1,2 and 3 applies for $\ell^{\prime *}$ as well. Note that as long as case 3.2 is true, we always obtain a new negative cycle and the whole process repeats.

Cases 1, 2 and 3 cover all the possible cases of negative cycle $\ell^{\star}$ for all of which we proved that there exists a balancing server reallocation. Putting everything in a nutshell, we proved that if the policy $\pi$ is not maximum weighted matching at 
time slot $t$ there exists a negative cycle in the graph obtained by the taking the union of the matchings of $\pi$ and MWM (i.e., $U$ ). We proved that by reallocation of the servers involved in the negative cycle, we always can find a balancing server reallocation for policy $\pi$.

\section{D.3 Proof of Lemma 5.3}

Proof: Fix any arbitrary policy $\pi \in \Pi_{t}^{h}$ and any arbitrary sample path

$$
\omega=(x(0), c(1), a(1), x(1), c(2), a(2), x(2), \ldots)
$$

of the underlying random variables $(X(0), C(1), A(1), X(1), C(2), A(2), X(2), \ldots)$. We apply the coupling method to construct from $\omega$ a new sample path

$$
\tilde{\omega}=(\tilde{x}(0), \tilde{c}(1), \tilde{a}(1), \tilde{x}(1), \tilde{c}(2), \tilde{a}(2), \tilde{x}(2), \ldots)
$$

resulting in a new sequence of random variables $(\tilde{X}(0), \tilde{C}(1), \tilde{A}(1), \tilde{X}(1), \tilde{C}(2), \tilde{A}(2), \tilde{X}(2), \ldots)$ with $X(0)=\tilde{X}(0)$. Note that $X(0)$ is the queue length vector by which the system starts working. We denote the policy driven by the new sample path $\tilde{\omega}$ by $\tilde{\pi}$. In fact, we construct $\tilde{\omega}$ and $\tilde{\pi} \in \Pi_{t}^{h-1}$ in such a fashion that for all the sample paths and all time slots we have $\tilde{x}(t) \preceq_{p} x(t)$. Therefore, for any $f \in \mathcal{F}$ we have $f(\tilde{x}(t)) \leq f(x(t))$ for all $t$. Since processes $\{(C(t), A(t))\}_{t=1}^{\infty}$ and $\{(\tilde{C}(t), \tilde{A}(t))\}_{t=1}^{\infty}$ are the same in distribution (these processes are permutation invariant), the process $\{f(\tilde{X}(t))\}$ obtained by applying policy $\tilde{\pi}$ to the system is stochastically smaller than $\{f(X(t))\}$, i.e., $\{f(\tilde{X}(t))\} \leq_{s t}\{f(X(t))\}$ and $\tilde{\pi}$ dominates $\pi$.

Therefore, in the following, our goal would be to construct $\tilde{\pi}$ and $\tilde{\omega}$ such that $\tilde{x}(t) \preceq_{p} x(t)$ is satisfied for all time slots. In the proof, we always use the tilde notation for all random variables that belong to the new system. The construction of $\tilde{\pi}$ is done in two steps:

Step 1: Construction of $\tilde{\pi}$ for $\tau \leq t$ : To construct the new sample path $\tilde{\omega}$ we let the arrival, connectivity and the policy be the same as the first system until time slot $t-1$, i.e., $\tilde{c}(\tau)=c(\tau), \tilde{a}(\tau)=a(\tau)$ and $I^{(\pi)}(\tau)=I^{(\tilde{\pi})}(\tau)$ for $\tau \leq t-1$. Thus, the resulting queue lengths at the beginning of time slot $t$ (or at the end of time slot 
$t-1)$ are equal, i.e., $\tilde{x}(t-1)=x(t-1)$. We now consider the construction of $\tilde{\omega}$ and $\tilde{\pi}$ for time slot $t$. For this time slot, one of the following two cases may apply.

1. The distance of policy $\pi$ to $\Pi_{t}$ is less that $h$ balancing server reallocations. Therefore $\pi \in \Pi_{t}^{h-1}$ and we let $\tilde{c}(t)=c(t)$ and $\tilde{a}(t)=a(t)$ and $I^{(\tilde{\pi})}(t)=I^{(\pi)}(t)$. Therefore, $\tilde{x}(t)$ and $x(t)$ are equal in both sample path $\omega$ and $\tilde{\omega}$. Thus, $\tilde{x}(t) \preceq$ $x(t)$.

2. The distance of policy $\pi$ to $\Pi_{t}$ is exactly $h$ balancing server reallocations. Since $\pi \in \Pi_{t}^{h}$ and $h>0$, according to Lemma 5.2 there exists a balancing server reallocation such that either $\mathbf{C 1}$ or $\mathbf{C 2}$ is satisfied. Thus, we consider two cases:

Case 2.1: After applying the balancing reallocation, condition $\mathbf{C 1}$ is satisfied. In other words, there exists a matching such that if applied on the queue length $\tilde{x}(t-1)=x(t-1)$ at time slot $t$, we get $\tilde{x}^{\prime}(t)$ such that $\tilde{x}^{\prime}(t) \leq x^{\prime}(t)$. We call such a matching by $I^{(\tilde{\pi})}(t)$. In this case, we let $\tilde{c}(t)=c(t)$ and $\tilde{a}(t)=a(t)$ and we apply $I^{(\tilde{\pi})}(t)$ at time slot $t$, i.e., arrivals and connectivities are the same in both systems and policy $\tilde{\pi}$ acts at time slot $t$. So, we can easily check that $\tilde{x}(t) \leq x(t)$ and therefore $\tilde{x}(t) \preceq x(t)$.

Case 2.2: After applying the balancing reallocation, condition C2 is satisfied. In other words, there exists a matching such that if applied on the system at time slot $t$, we get $\tilde{x}^{\prime}(t)$ which is different from $x^{\prime}(t)$ in two elements $m$ and $n$ such that $x_{n}^{\prime}(t)<\tilde{x}_{n}^{\prime}(t) \leq \tilde{x}_{m}^{\prime}(t)<x_{m}^{\prime}(t)$ and the following constraints are satisfied: $\tilde{x}_{n}^{\prime}(t)=x_{n}^{\prime}(t)+1$ and $\tilde{x}_{m}^{\prime}(t)=x_{m}^{\prime}(t)-1$. We call such a matching by $I^{(\tilde{\pi})}(t)$. In this case, we let $\tilde{c}(t)=c(t)$ and $\tilde{a}(t)=a(t)$ and we apply $I^{(\tilde{\pi})}(t)$ at time slot $t$, i.e., arrivals and connectivities are the same in both systems and policy $\tilde{\pi}$ acts at time slot $t$. We consider all the following conditions for arrivals to queues $m$ and $n$ as follows:

- If there is no arrival or there is an arrival to both queues $m$ and $n$ (i.e., $a_{m}(t)=a_{n}(t)=0$ or $\left.a_{m}(t)=a_{n}(t)=1\right)$, we conclude that $\tilde{x}(t)$ and $x(t)$ satisfy condition D3. Thus, $\tilde{x}(t) \preceq x(t)$.

- If there is an arrival to queue $m$ but not $n$ (i.e., $a_{m}(t)=1, a_{n}(t)=0$ ), we conclude that $\tilde{x}(t)$ and $x(t)$ satisfy condition D3. Thus, $\tilde{x}(t) \preceq x(t)$. 
- If there is an arrival to queue $n$ but not $m$ (i.e., $a_{m}(t)=0, a_{n}(t)=1$ ) and $\tilde{x}_{m}(t)=\tilde{x}_{n}(t)$, we conclude that $\tilde{x}(t)$ and $x(t)$ satisfy condition D2. Thus, $\tilde{x}(t) \preceq x(t)$.

- If there is an arrival to queue $n$ but not $m$ (i.e., $a_{m}(t)=0, a_{n}(t)=1$ ) and $\tilde{x}_{n}(t)<\tilde{x}_{m}(t)$, we conclude that $\tilde{x}(t)$ and $x(t)$ satisfy condition D3. Thus, $\tilde{x}(t) \preceq x(t)$.

In all the cases we can see that $\tilde{x}(t) \preceq x(t)$.

Note that the obtained policy $\tilde{\pi}$ belongs to $\Pi_{t}^{h-1}$ as we applied a balancing server reallocation to the matching employed in $\pi$ at time slot $t$.

Step 2: Construction of $\tilde{\pi}$ for $\tau>t$ : In this step, we focus on construction of $\tilde{\omega}$ and $\tilde{\pi}$ for $\tau>t$. We will employ mathematical induction to achieve this goal. In particular, we assume that $\tilde{\omega}$ and $\tilde{\pi}$ are constructed up to time slot $\tau(\tau \geq t)$ such that $\tilde{x}(\tau) \preceq x(\tau)$ i.e., one of the conditions D1, D2 and D3 is satisfied for $x(\tau)$ and $\tilde{x}(\tau)$. We will prove that policy $\tilde{\pi}$ and sample path $\tilde{\omega}$ can be constructed such that $\tilde{x}(\tau+1) \preceq x(\tau+1)$ (i.e., one of the conditions D1, D2 and D3 is satisfied for $x(\tau+1)$ and $\tilde{x}(\tau+1))$. Accordingly, we consider three cases corresponding to each condition D1, D2 or D3 at time slot $\tau$ :

Case 1: $\tilde{x}(\tau) \leq x(\tau)$. In this case, the construction of $\tilde{\omega}$ and $\tilde{\pi}$ at time slot $\tau+1$ is straightforward. We let $\tilde{c}(\tau+1)=c(\tau+1)$ and $\tilde{a}(\tau+1)=a(\tau+1)$ and $I^{(\tilde{\pi})}(\tau+1)=I^{(\pi)}(\tau+1)$. Thus, $\tilde{x}(\tau+1) \leq x(\tau+1)$ and therefore $\tilde{x}(\tau+1) \preceq x(\tau+1)$.

Case 2: $\tilde{x}(\tau)$ is obtained from $x(\tau)$ by permutation of two distinct elements $m$ and $n$. In this case, we let $\tilde{c}_{n, k}(\tau+1)=c_{m, k}(\tau+1)$ and $\tilde{c}_{m, k}(\tau+1)=c_{n, k}(\tau+1)$ for $k=1,2, \ldots, K ; \tilde{c}_{i, k}(\tau+1)=c_{i, k}(\tau+1)$ for all $i \in \mathcal{N}, i \neq n, m$ and $k=1,2, \ldots, K$; $\tilde{a}_{n}(\tau+1)=a_{m}(\tau+1), \tilde{a}_{m}(\tau+1)=a_{n}(\tau+1)$ and $\tilde{a}_{i}(\tau+1)=a_{i}(\tau+1)$ for $i \in \mathcal{N}$, $i \neq n, m$. Suppose that $I^{(\pi)}(\tau+1)=\left(I_{n, k}^{(\pi)}(\tau+1)\right) \forall n \in \mathcal{N}, k \in \mathcal{K}$ be the applied matching by policy $\pi$ at time slot $\tau+1$. We construct $I^{(\tilde{\pi})}(\tau+1)$ as follows: Let $I_{i, k}^{(\tilde{\pi})}(\tau+1)=I_{i, k}^{(\pi)}(\tau+1)$ for $i \in \mathcal{N}, i \neq n, m$ and also let $I_{n, k}^{(\tilde{\pi})}(\tau+1)=I_{m, k}^{(\pi)}(\tau+1)$ and $I_{m, k}^{(\tilde{\pi})}(\tau+1)=I_{n, k}^{(\pi)}(\tau+1)$ for $k=1,2, \ldots, K$. As a result, $\tilde{x}(\tau+1)$ and $x(\tau+1)$ satisfy condition D2 at time slot $\tau+1$ and therefore $\tilde{x}(\tau+1) \preceq x(\tau+1)$.

Case 3: $\tilde{x}(\tau)$ is obtained from $x(\tau)$ by performing a balancing interchange of two distinct elements $m$ and $n$ as defined in condition D3. In particular, $\tilde{x}(\tau)$ and $x(\tau)$ are different in only two elements $n$ and $m$ such that $x_{n}(\tau)<\tilde{x}_{n}(\tau) \leq \tilde{x}_{m}(\tau)<x_{m}(\tau)$ and the following constraints are satisfied: $\tilde{x}_{n}(\tau)=x_{n}(\tau)+1$ and $\tilde{x}_{m}(\tau)=x_{m}(\tau)-1$. In this case, we consider the following sub-cases: 
Sub-case 3.1: $\tilde{x}_{n}(\tau)<\tilde{x}_{m}(\tau)-1$ : In this case, we let $\tilde{c}(\tau+1)=c(\tau+1)$ and $\tilde{a}(\tau+1)=a(\tau+1)$ and we let $I^{(\tilde{x})}(\tau+1)=I^{(\pi)}(\tau+1)$. Thus, if $x_{n}(\tau)=0$ and queue $n$ is serviced, condition D1 is satisfied at $\tau+1$. Otherwise, $\tilde{x}(\tau+1)$ is obtained from $x(\tau+1)$ by performing a balancing interchange of elements $m$ and $n$. Therefore $\tilde{x}(\tau+1) \preceq x(\tau+1)$.

Sub-case 3.2: $\tilde{x}_{n}(\tau)=\tilde{x}_{m}(\tau)-1$ : In this case again we let $\tilde{c}(\tau+1)=c(\tau+1)$ and $\tilde{a}(\tau+1)=a(\tau+1)$ and we let $I^{(\tilde{\pi})}(\tau+1)=I^{(\pi)}(\tau+1)$. Thus, if $x_{n}(\tau)=0$ and queue $n$ is serviced, condition D1 is satisfied at $\tau+1$. If queue $m$ gets service, queue $n$ does not get service, there is an arrival to queue $n$ and no arrival to queue $m$, then $\tilde{x}_{n}(\tau+1)=x_{m}(\tau+1)$ and $\tilde{x}_{m}(\tau+1)=x_{n}(\tau+1)$. Therefore, $\tilde{x}(\tau+1)$ and $x(\tau+1)$ satisfy condition $\mathbf{D} 2$ and $\tilde{x}(\tau+1) \preceq x(\tau+1)$. Otherwise, $\tilde{x}(\tau+1)$ and $x(\tau+1)$ satisfy condition $\mathbf{D} 3$ and $\tilde{x}(\tau+1) \preceq x(\tau+1)$.

Sub-case 3.3: $\tilde{x}_{n}(\tau)=\tilde{x}_{m}(\tau)$ : In this case, we let $\tilde{c}(\tau+1)=c(\tau+1)$ and $I^{(\tilde{\pi})}(\tau+1)=I^{(\pi)}(\tau+1)$. Now, we consider the following cases to determine the arrivals at time slot $\tau+1$.

- If $x_{n}(\tau)>0$ and both queues $m$ and $n$ or none of them get service at time slot $\tau+1$, we let $\tilde{a}(\tau+1)=a(\tau+1)$. Therefore, if $a_{m}(\tau+1)=0$ and $a_{n}(\tau+1)=1$, $\tilde{x}(\tau+1)$ and $x(\tau+1)$ satisfy condition D2 and thus $\tilde{x}(\tau+1) \preceq x(\tau+1)$. Otherwise, $\tilde{x}(\tau+1)$ and $x(\tau+1)$ satisfy condition D3 and thus $\tilde{x}(\tau+1) \preceq$ $x(\tau+1)$.

- If $x_{n}(\tau)>0$ and queue $n$ gets service at time slot $\tau+1$ and queue $m$ does not get service at time slot $\tau+1$, we let $\tilde{a}(\tau+1)=a(\tau+1)$. Therefore, $\tilde{x}(\tau+1)$ and $x(\tau+1)$ satisfy condition $\mathbf{D 3}$ and thus $\tilde{x}(\tau+1) \preceq x(\tau+1)$.

- If $x_{n}(\tau)>0$ and queue $m$ gets service at time slot $\tau+1$ and queue $n$ does not get service at time slot $\tau+1$, we let $\tilde{a}_{m}(\tau+1)=a_{n}(\tau+1)$ and $\tilde{a}_{n}(\tau+1)=a_{m}(\tau+1)$ and $\tilde{a}_{i}(\tau+1)=a_{i}(\tau+1)$ for $i \in \mathcal{N}$ and $i \neq m, n$. Therefore, $\tilde{x}(\tau+1)$ and $x(\tau+1)$ satisfy condition $\mathbf{D} 2$ and thus $\tilde{x}(\tau+1) \preceq x(\tau+1)$.

- If $x_{n}(\tau)=0$ and queue $n$ gets service at time slot $\tau+1$ (although it does not have any packet to be served), we let $\tilde{a}(\tau+1)=a(\tau+1)$. Therefore, $\tilde{x}(\tau+1)$ and $x(\tau+1)$ satisfy condition $\mathbf{D 1}$ and thus $\tilde{x}(\tau+1) \preceq x(\tau+1)$.

- If $x_{n}(\tau)=0$ and queue $m$ gets service at time slot $\tau+1$ and queue $n$ does not get service at time slot $\tau+1$, we let $\tilde{a}_{m}(\tau+1)=a_{n}(\tau+1)$ and $\tilde{a}_{n}(\tau+1)=a_{m}(\tau+1)$ 
and $\tilde{a}_{i}(\tau+1)=a_{i}(\tau+1)$ for $i \in \mathcal{N}$ and $i \neq m, n$. Therefore, $\tilde{x}(\tau+1)$ and $x(\tau+1)$ satisfy condition D2 and thus $\tilde{x}(\tau+1) \preceq x(\tau+1)$.

- If $x_{n}(\tau)=0$ and neither queue $m$ nor $n$ gets service at time slot $\tau+1$, we let $\tilde{a}(\tau+1)=a(\tau+1)$. If $a_{m}(\tau+1)=0$ and $a_{n}(\tau+1)=1, \tilde{x}(\tau+1)$ and $x(\tau+1)$ satisfy condition D2 and thus $\tilde{x}(\tau+1) \preceq x(\tau+1)$. Otherwise, $\tilde{x}(\tau+1)$ and $x(\tau+1)$ satisfy condition D3 and thus $\tilde{x}(\tau+1) \preceq x(\tau+1)$.

Note that the above cases cover all the possible cases for all of which we constructed $\tilde{\omega}$ and $\tilde{\pi}$ such that $\tilde{x}(\tau+1) \preceq x(\tau+1)$.

According to steps 1 and 2, from any sample path $\omega$ and any arbitrary policy $\pi \in \Pi_{t}^{h}$ we can construct a sample path $\tilde{\omega}$ and a policy $\tilde{\pi} \in \Pi_{t}^{h-1}$ such that at all time slots we have $\tilde{x}(t) \preceq_{p} x(t)$. Therefore, $f(\tilde{x}(t)) \leq f(x(t))$. Consequently, the process $\{f(\tilde{X}(t))\}$ obtained by applying policy $\tilde{\pi}$ to the system is stochastically smaller that $\{f(X(t))\}$, i.e., $\{f(\tilde{X}(t))\} \leq_{s t}\{f(X(t))\}$ and therefore $\tilde{\pi} \in \Pi_{t}^{h-1}$ dominates $\pi \in \Pi_{t}^{h}$.

\section{D.4 Proof of Lemma 5.3 for the System with Ran- dom Service Failures}

Proof: The only difference of the proof in this case from the proof of Lemma 5.3 is that we have to consider random variables $Q_{n, k}(t) \forall n \in \mathcal{N}, \forall k \in \mathcal{K}$ in our dynamic coupling argument. Therefore, the arbitrary sample path has the form

$$
\omega=(x(0), c(1), q(1), a(1), x(1), c(2), q(2), a(2), x(2), \ldots)
$$

and we apply the coupling method to construct from $\omega$ a new sample path

$$
\tilde{\omega}=(\tilde{x}(0), \tilde{c}(1), \tilde{q}(1), \tilde{a}(1), \tilde{x}(1), \tilde{c}(2), \tilde{q}(2), \tilde{a}(2), \tilde{x}(2), \ldots)
$$

resulting in a new sequence of random variables

$$
(\tilde{X}(0), \tilde{C}(1), \tilde{Q}(1), \tilde{A}(1), \tilde{X}(1), \tilde{C}(2), \tilde{Q}(2), \tilde{A}(2), \tilde{X}(2), \ldots)
$$

with $X(0)=\tilde{X}(0)$. We will follow the same approach and consider the same cases. 
Before we proceed to the details we introduce the following complementary notation. Suppose that $s_{n}^{(n)}(t)$ denotes the index of the server assigned to queue $n$ at time slot $t$ by policy $\pi$. Note that $s_{n}^{(\pi)}(t) \in \mathcal{K}$ or it is empty.

Step 1: Construction of $\tilde{\pi}$ for $\tau \leq t$ : In this case, as in the proof of Lemma 5.3 we first consider $\tau \leq t-1$. For those slots, we let all $\tilde{c}(\tau), \tilde{q}(\tau), \tilde{a}(\tau)$ and $I^{(\tilde{\pi})}(\tau)$ to be the same in both systems i.e., $\tilde{c}(\tau)=c(\tau), \tilde{q}(\tau)=q(\tau), \tilde{a}(\tau)=a(\tau)$ and $I^{(\tilde{\pi})}(\tau)=I^{(\pi)}(\tau)$ and therefore we have $\tilde{x}(t-1)=x(t-1)$.

For time slot $t$, we again consider the following cases.

1. The distance of policy $\pi$ to $\Pi_{t}$ is less that $h$ balancing server reallocations. In this case, we act the same as what we did in the proof of Lemma 5.3 and also let $\tilde{q}(t)=q(t)$. Thus, $\tilde{x}(t) \preceq x(t)$.

2. The distance of policy $\pi$ to $\Pi_{t}$ is exactly $h$ balancing server reallocations. Since $\pi \in \Pi_{t}^{h}$ and $h>0$, according to Lemma 5.2 there exists a balancing server reallocation such that either $\mathbf{C 1}$ or $\mathbf{C 2}$ is satisfied. Thus, we consider two cases.

Case 2.1: After applying the balancing reallocation, condition $\mathbf{C 1}$ is satisfied. In other words, there exists a matching $I^{(\tilde{\pi})}(t)$ such that if applied on the queue lengths $\tilde{x}(t-1)=x(t-1)$, we get $\tilde{x}^{\prime}(t)$ such that $\tilde{x}^{\prime}(t) \leq x^{\prime}(t)$. Note that if a non-empty queue is serviced under policy $I^{(\pi)}(t)$, it should also get service under policy $\tilde{\pi}$. Otherwise, the condition $\tilde{x}^{\prime}(t) \leq x^{\prime}(t)$ will be violated. In this case, we let $\tilde{c}(t)=c(t)$ and $\tilde{a}(t)=a(t)$ and we apply $I^{(\tilde{\pi})}(t)$ at time slot $t$. For any non-empty queue $n$ that is serviced under both policies $\pi$ and $\tilde{\pi}$ we let $\tilde{q}_{n, s_{n}^{(\pi)}(t)}(t)=q_{n, s_{n}^{(\pi)}(t)}(t)$ and $\tilde{q}_{n, s_{n}^{(\pi)}(t)}(t)=q_{n, s_{n}^{(\pi)}(t)}(t)$. In other words, we let each non-empty queue $n$ which was being serviced in both systems experience the same service failure. For other variables $\tilde{q}_{n, k}(t)$ we let $\tilde{q}_{n, k}(t)=q_{n, k}(t)$. Then, we can easily check that $\tilde{x}(t) \leq x(t)$ and therefore $\tilde{x}(t) \preceq x(t)$.

Case 2.2: After applying the balancing reallocation, condition C2 is satisfied. In other words, there exists a matching such that if applied on the system at time slot $t$, we get $\tilde{x}^{\prime}(t)$ which is different from $x^{\prime}(t)$ in two elements $m$ and $n$ such that $x_{n}^{\prime}(t)<\tilde{x}_{n}^{\prime}(t) \leq \tilde{x}_{m}^{\prime}(t)<x_{m}^{\prime}(t)$ and the following constraints are satisfied; $\tilde{x}_{n}^{\prime}(t)=x_{n}^{\prime}(t)+1$ and $\tilde{x}_{m}^{\prime}(t)=x_{m}^{\prime}(t)-1$. We call such a matching by $I^{(\tilde{\pi})}(t)$. Note that in this case, each queue (other than $m$ and $n$ ) which is (is not) receiving service under $\pi$ is (is not) also receiving service under $\tilde{\pi}$. Queue $n$ is receiving 
service under $\pi$ and queue $m$ is not. Queue $n$ is not receiving service under $\tilde{\pi}$ and queue $m$ is. In this case, we let $\tilde{c}(t)=c(t)$ and $\tilde{a}(t)=a(t)$ and we apply $I^{(\tilde{\pi})}(t)$ at time slot $t$. We let $\tilde{q}_{i, s_{i}^{(\bar{\pi})}(t)}(t)=q_{i, s_{i}^{(\pi)}(t)}(t)$ and $\tilde{q}_{i, s_{i}^{(\pi)}(t)}(t)=q_{i, s_{i}^{(\bar{\pi})}(t)}(t)$ for any queue $i$ which is receiving service under both matchings $I^{(\pi)}(t)$ and $I^{(\tilde{\pi})}(t)$. We also let $\tilde{q}_{m, s_{m}^{(\tilde{\pi})}(t)}(t)=q_{n, s_{n}^{(\pi)}(t)}(t), \tilde{q}_{n, s_{n}^{(\pi)}(t)}(t)=q_{m, s_{m}^{(\bar{m})}(t)}(t)$ and for other failure variables we let $\tilde{q}_{n, k}(t)=q_{n, k}(t)$. By such coupling of the service success/failure random variables we will consider the following cases for arrivals and service failures:

- If $q_{n, s_{n}^{(\pi)}(t)}(t)=0$, we let $\tilde{a}(t)=a(t)$ and therefore, $\tilde{x}(t)=x(t)$ which implies $\tilde{x}(t) \preceq x(t)$.

- If $q_{n, s_{n}^{(\pi)}(t)}(t)=1$, then we can use the same coupling argument on the arrival processes as what we did in the proof of Lemma 5.3 and conclude that $\tilde{x}(t) \preceq x(t)$. We omit the argument to avoid redundant discussion.

Note that the obtained policy $\tilde{\pi}$ belongs to $\Pi_{t}^{h-1}$ as we applied a balancing server reallocation to the matching employed in $\pi$ at time slot $t$.

Step 2: Construction of $\tilde{\pi}$ for $\tau>t$ : In this step, the same as the proof of Lemma 5.3 we use mathematical induction to construct policy $\tilde{\pi}$ for $\tau>t$. Therefore, suppose that $\tilde{\omega}$ and $\tilde{\pi}$ are constructed up to time slot $\tau(\tau \geq t)$ such that $\tilde{x}(\tau) \preceq x(\tau)$. Therefore, one of the conditions D1, D2 and D3 is satisfied for $x(\tau)$ and $\tilde{x}(\tau)$ as follows.

Case 1: $\tilde{x}(\tau) \leq x(\tau)$. In this case, the construction of $\tilde{\omega}$ and $\tilde{\pi}$ at time slot $\tau+1$ is straightforward. We let $\tilde{c}(\tau+1)=c(\tau+1), \tilde{q}(\tau+1)=q(\tau+1), \tilde{a}(\tau+1)=a(\tau+1)$ and policy $I^{(\tilde{\pi})}(\tau+1)=I^{(\pi)}(\tau+1)$. Thus, $\tilde{x}(\tau+1) \leq x(\tau+1)$ and therefore $\tilde{x}(\tau+1) \preceq x(\tau+1)$.

Case 2: $\tilde{x}(\tau)$ is obtained from $x(\tau)$ by permutation of two distinct elements $m$ and $n$. In this case, we couple the random variables $c(\tau+1), a(\tau+1)$ and construct matching $I^{(\tilde{\pi})}(\tau+1)$ the same as what we did in case 2 of step 2 in the proof of Lemma 5.3. In addition to these settings, we let $\tilde{q}_{m, k}(\tau+1)=q_{n, k}(\tau+1)$ and $\tilde{q}_{n, k}(\tau+1)=q_{m, k}(\tau+1)$ for $k=1,2, \ldots, K$ and also $\tilde{q}_{i, k}(\tau+1)=q_{i, k}(\tau+1)$ for all $i \in \mathcal{N}, i \neq n, m$ and $k=1,2, \ldots, K$. By doing such a coupling, we conclude that $\tilde{x}(\tau+1)$ and $x(\tau+1)$ satisfy condition D2 at time slot $\tau+1$ and therefore $\tilde{x}(\tau+1) \preceq x(\tau+1)$.

Case 3: $\tilde{x}(\tau)$ is obtained from $x(\tau)$ by performing a balancing interchange of two 
distinct elements $m$ and $n$ as defined in condition D3. In particular, $\tilde{x}(\tau)$ and $x(\tau)$ are different in only two elements $n$ and $m$ such that $x_{n}(\tau)<\tilde{x}_{n}(\tau) \leq \tilde{x}_{m}(\tau)<x_{m}(\tau)$ and the following constraints are satisfied; $\tilde{x}_{n}(\tau)=x_{n}(\tau)+1$ and $\tilde{x}_{m}(\tau)=x_{m}(\tau)-1$. In this case, with the same argument as we did in the proof of Lemma 5.3, we can check that $\tilde{x}(\tau+1)$ and $x(\tau+1)$ satisfy one of the conditions D1-D3. We omit the details to avoid redundant discussion. 